Metal Complex-Based Templates and Nanostructures for Magnetic Resonance/Optical Multimodal

\title{
Imaging Agents
}


METAL COMPLEX-BASED TEMPLATES

AND NANOSTRUCTURES FOR

MAGNETIC RESONANCE/OPTICAL

MULTIMODAL IMAGING AGENTS 
The research described in this thesis was carried out within the Biomolecular Nanofabrication group (BnT), MESA+ Institute for Nanotechnology, University of Twente.

Committee members:

Chairman: Prof. Dr. Ir. W. Steenbergen Universiteit Twente

Promotoren: Prof. Dr. Ir. D.N. Reinhoudt Universiteit Twente Prof. Dr. J.J.L.M. Cornelissen Universiteit Twente

\section{Assistant-promotor:}

Prof. Dr. A.H. Velders

Landbouwuniversiteit Wageningen

Members: Prof. Dr. Ir. J. Huskens

Universiteit Twente

Prof. Dr. J.L. Herek

Universiteit Twente

Prof. Dr. L. de Cola

Westfälische WilhelmsUniversität Münster

Prof. Dr. R.J.M. Nolte

Radboud Universiteit

Nijmegen

Prof. Dr. J.F.J. Engbersen

Universiteit Twente

Cover Art: Lonely in the middle of nothing, by J. Galindo Millán

Publisher: Wöhrmann Print Services, Zutphen, The Netherlands

ISBN: 978-90-365-3448-2

DOI: 10.3990/1.9789036534482

(C) Jealemy Josefina Galindo Millán, Enschede 2012

No part of this work may be reproduced by print, photocopy or any other means without the permission in writing of the author. 


\title{
IMETAL COMPLEX-BASED TFMPLATFS AND \\ NANOSTRUCTURES FOR MAGNFTIC
}

RFSONANCF/OPTICAI MULTIMODAL IMAGING AGENTS

\author{
PROEFSCHRIFT
}

ter verkrijging van

de graad van doctor aan de Universiteit Twente,

op gezag van de rector magnificus,

Prof. Dr. H. Brinksma,

volgens besluit van het College voor Promoties

in het openbaar te verdedigen

op vrijdag 2 november 2012 om 16.45 uur

door

Jealemy Josefina Galindo Millán

Geboren op 9 maart 1981

te Caracas, Venezuela 
Dit proefschrift is goedgekeurd door:

Promotoren:

Prof. Dr. Ir. D.N. Reinhoudt

Prof. Dr. J.J.L.M. Cornelissen

Assistant-promotor:

Prof. Dr. A.H. Velders 
Nan-in, a Japanese master during the Meiji era (1868-1912), received a university professor who came to inquire about Zen.

Nan-in served tea. He poured his visitor's cup full, and then kept on pouring. The professor watched the overflow until he no longer could restrain himself.

"It is overfull. No more will go in!" "Like this cup," Nan-in said, "you are full of your own opinions and speculations. How can I show you Zen unless you first empty your cup?"

Zen Koan \#1 



\section{TABLE OF CONTENTS}

CHAPTER 1: INTRODUCTION

1.1 GENERAL INTRODUCTION

1.2 REFERENCES

CHAPTER Z: MULTIMODAL AGENTS FOR

MAGNETIC RESONANCE AND FLUORESCENCE IMAGING 7

2.1 INTRODUCTION: MOLECULAR IMAGING

2.2 MULTIMODAL IMAGING 10

2.3 MAgnetic RESONANCE AND Optical IMAGING 13

2.4 StRategies for Magnetic Resonance/Optical MULtimodal IMAGING AGENTS $\quad 30$

2.5 CONCLUSIONS 56

2.6 REFERENCES 56

CHAPTFR 3: MULTIFUNCTIONAL HYBRID SILVER NANOSTRUCTURES GROWN USING A POLYAIMINO CARBOXYLIC ACID SCAFFOLD $\quad 65$

$\begin{array}{lll}3.1 & 66\end{array}$

$\begin{array}{lll}3.2 & \text { DESIGN AND SYNTHESIS } & 67\end{array}$

3.3 RESULTS AND DISCUSSION 69

$\begin{array}{lll}3.4 & \text { CONCLUSIONS } & 81\end{array}$

3.5 EXPERIMENTAL 82

3.6 REFERENCES 86

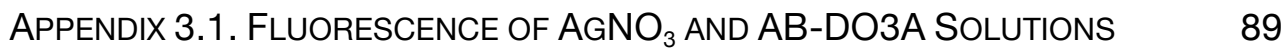

APPENDIX 3.2. AMPLIFICATION OF MALDI-MS REGIONS

$2\left[\mathrm{AG}_{2} \mathrm{~L}-\mathrm{H}+\mathrm{K}+\mathrm{M}\right]^{-} \mathrm{AND} 3\left[\mathrm{AG}_{2}-\mathrm{H}+\mathrm{K}+2 \mathrm{M}\right]^{-} \quad 90$

APPENDIX 3.3. TITRATION OF AB-DO3A WITH $\mathrm{AG}^{+}$FOLLOWED

BY ${ }^{1} \mathrm{H}-\mathrm{NMR} \quad 91$ 
CHAPTER 4: SELF-ASSEMBLY TRIGGERFD BY

SELF-ASSEIMBLY: PROTEIN CAGE ENCAPSULATED

MICELLES VISUALIZED BY MRI

95

4.1 INTRODUCTION

96

4.2 DESIGN AND SYNTHESIS

98

4.3 RESULTS AND DISCUSSION

100

4.4 CONCLUSIONS

109

4.5 EXPERIMENTAL

110

4.6 REFERENCES

116

APPENDIX 4.1. ENCAPSULATION OF ZNPC DYE

APPENDIX 4.2. TXRF DATA, CAPSID CONCENTRATION AND

ENCAPSULATION EFFICIENCY CALCULATIONS

APPENDIX 4.3. TEM ANALYSIS

124

APPENDIX 4.4. MRI DATA

CHAPTER 5: MULTIMODAL GOLD NANORODS FOR

MAGNETIC RESONANCE AND FLUORESCENCE IMAGING 127

5.1 INTRODUCTION

128

5.2 DESIGN AND SYNTHESIS

129

5.3 RESULTS AND DISCUSSION

134

5.4 CONCLUSIONS

148

5.5 EXPERIMENTAL

149

5.6

REFERENCES

159

Appendix 5.1. Calculation of the Critical Aggregation

CONCENTRATION (CAC) OF $\mathrm{GD}^{3+}$-SUDDO3A 
CHAPTER 6: FUTURE PERSPECTIVES: METAL COMPLEX-

BASED TEMPLATES IN CARBON-BASED SCAFFOLDS

AS MAGNETIC RESONANCE/OPTICAL

IMULTIMODAL IMAGING AGENTS

$6.1 \quad$ INTRODUCTION 166

6.2 AN ANTHRACENE-CORE MULTIMOdAL AGENT FOR MAGNETIC RESONANCE AND FLUORESCENCE IMAGING

6.3 CARBON NANOTUBES (CNTS) FOR MULTIMODAL MOLECULAR IMAGING: A PRELIMINARY STUDY

6.4 Challenges in METAL COMPLEX-BASEd TEMPLATES FOR MAgnetic Resonance/Optical Multimodal IMAging AgentS

6.5 WHAT'S (REALLY) NEXT?

6.6 CONCLUSIONS

6.7 EXPERIMENTAL

6.8

REFERENCES

APPENDIX 6.1. CHARACTERIZATION OF THE BUILDING BLOCKS 3 AND 8 

CHAPTER 1

INTRODUCTION 


\subsection{General Introduction}

Molecular imaging has rapidly emerged as a discipline dedicated to address the need for accurate and rapid visualization of the human body in a, desirably, non-invasive way. ${ }^{1-3}$ Today, the various imaging modalities used in the clinic include those based on nuclear techniques (positron emission tomography -PET- and single photon emission computed tomography -SPECT-), proton relaxivity (magnetic resonance imaging MRI-) and optical phenomena (e.g., fluorescence, absorption and bioluminescence).$^{3-5}$ Despite the valuable information these imaging modalities provide, they fail to discriminate between physiologically/pathologically different tissues. To overcome this issue imaging agents (IAs), designed to aid image contrast without perturbing metabolic processes, are used. ${ }^{6}$

As more metabolic paths of the human body are unveiled by novel IAs and state-of-the-art techniques, ${ }^{3,5,7}$ a door has opened up for chemists, biologists and physicians to explore new approaches towards molecular imaging. Yet, a more conservative approach, where priority is given to well-established imaging modalities, is still preferred. However, the wellknown limited spatial resolution and/or low sensitivity these techniques suffer from have led to efforts being focused on the optimization of their synergy rather than on individual advances. Whilst several examples in the literature nicely illustrate the value of this multimodal approach, ${ }^{8-10}$ challenges and issues still remain.

Today, there is a wide variety of high-performance IAs, as well as a large number of well-studied, commercially available dyes, contrast and targeting agents. Many of these IAs have successfully been combined with nanomaterials and self-assembling systems, thereby leading to a large 
"molecular imaging library", where endless combinations are possible. Despite the innumerable successful examples described in the literature, ${ }^{8,10-17}$ the question remains: how can we engineer simple, fast, cheap and safe multimodal IAs? Inspired by the need for novel, versatile multimodal probes, the aim of this thesis is to postulate new approaches directed towards simple and functional agents for MR and fluorescence imaging. The multimodal IAs described in this thesis derive from combinations of (novel) metal complex-based ligands with nanomaterials. The versatility of this approach is reflected in the fact that the ligands presented here can be used as templates/scaffolds for the growth of nanostructures, for other imaging techniques (by complexation with various metals) and can also be combined with an endless number of nanomaterials.

In Chapter 2, a literature survey providing a general overview of multimodal IAs, with emphasis in general contrast agent and surface chemistry combined with nanomaterials, is presented. While several strategies are briefly highlighted, this survey mostly deals with multimodal probes where Gadolinium $\left(\mathrm{Gd}^{3+}\right)$ complexes are used for magnetic resonance, combined with fluorescence as the optical imaging technique.

The aqueous-based, one-step synthesis of hybrid silver nanostructures (hAgNSs) grown using the polyamino carboxylic acid scaffold AB-DO3A is presented in Chapter 3. A feasible structure for these hAgNSs is depicted using UV-Vis absorbance spectroscopy, transmission electron microscopy (TEM), dynamic light scattering (DLS), NMR spectroscopy, mass spectrometry (MS) and fluorescence spectroscopy (lifetime and steadystate). 
Pure hierarchical self-assembly of protein cages of the cowpea chlorotic mottle virus (CCMV) around amphiphilic $\left(\mathrm{Gd}^{3+}\right)$-DOTAC10 micelles is described in Chapter 4. Encapsulation of the micelles by the viral nanoparticles was optically confirmed using an amphiphilic $\mathrm{Zn}^{2+}$ phthalocyanine $(\mathrm{ZnPc})$ dye and by visualization using MRI. The size and paramagnetic properties of these self-assembled protein cages were studied using TEM and MRI, respectively.

In Chapter 5, the synthesis and characterization of multimodal gold nanorods (MMAuNRs) for magnetic resonance and optical imaging are described. The gold nanostructures were modified by thiol-based ligand exchange using various PEG-SH, Gd ${ }^{3+}-$ SUDDO3A and Biotin-SH ratios. The biocompatibility of the MMAuNRs was studied by means of cell viability studies and the gold nanostructures were visualized by MRI and fluorescence microscopy.

In Chapter 6, the versatility of the metal complex-based template described in Chapter 3 (and similar to the one designed for Chapter 5) is demonstrated by its incorporation in carbon-based scaffolds such as small organic molecules and carbon nanotubes (CNTs). The synthetic strategies followed are described and the properties of these novel materials are investigated. Preliminary results illustrate the potential of the approach proposed, thereby introducing a short discussion on the challenges and future perspectives of the IAs presented in this thesis. 


\subsection{References}

(1) Galbán, C. J.; Galbán, S.; Van Dort, M. E.; Luker, G. D.; Bhojani, M. S.; Rehemtulla, A.; Ross, B. D. In Progress in Molecular Biology and Translational Science; Raymond, W. R., Ed.; Academic Press: 2010; Vol. Volume 95, p 237.

(2) Hausner, S. H. In Nanoplatform-Based Molecular Imaging; Chen, X., Ed.; John Wiley \& Sons, Inc.: 2011, p 1.

(3) Weissleder, R.; Mahmood, U. Radiology 2001, 219, 316.

(4) Brindle, K. Nat. Rev. Cancer 2008, 8, 94.

(5) Cassidy, P. J.; Radda, G. K. J. Royal Soc. Interface 2005, 2, 133.

(6) Seaman, M. E.; Contino, G.; Bardeesy, N.; Kelly, K. A. Expert Rev. Mol. Med. 2010, 12, 1.

(7) Weissleder, R.; Pittet, M. J. Nature 2008, 452, 580.

(8) Frullano, L.; Meade, T. J. Biol. Inorg. Chem. 2007, 12, 939.

(9) Jennings, L. E.; Long, N. J. Chem. Commun. 2009, 3511.

(10) Louie, A. Chem. Rev. 2010, 110, 3146.

(11) Cherry, S. R.; Louie, A. Y.; Jacobs, R. E. P. IEEE 2008, 96, 416.

(12) Culver, J.; Akers, W.; Achilefu, S. J. Nucl. Med. 2008, 49, 169.

(13) Hou, Y.; Hao, R. In Nanoplatform-Based Molecular Imaging; Chen, X., Ed.; John Wiley \& Sons, Inc.: 2011, p 529.

(14) Huang, W.-Y.; Davis, J. J. Dalton Trans. 2011, 40, 6087.

(15) Kim, J.; Piao, Y.; Hyeon, T. Chem. Soc. Rev. 2009, 38, 372.

(16) Mulder, W. J. M.; Strijkers, G. J.; van Tilborg, G. A. F.; Cormode, D. P.; Fayad, Z. A.; Nicolay, K. Acc. Chem. Res. 2009, 42, 904.

(17) Pinho, S. L. C.; Faneca, H.; Geraldes, C. F. G. C.; Rocha, J.; Carlos, L. D.; Delville, M.-H. Eur. J. Inorg. Chem. 2012, 2828. 
CHAPTER 1 


\section{CHAPTER 2}

\section{MULTIMODAL AGENTS FOR MAGNETIC RESONANCE AND FLUORESCENCE IMAGING}

\section{Abstract}

In medical diagnosis, combinations of different imaging modalities are often used in order to address the shortcomings of each individual technique while, at the same time, taking advantage of their individual strengths. In this chapter, a concise literature overview of various multimodal agents is presented, with particular interest in those combining magnetic resonance with optical imaging techniques. A selection of different multimodal imaging strategies explored until this day, based on advances in surface chemistry, contrast agent synthesis and nanotechnology, are discussed together with some theoretical background. 


\subsection{Introduction: Molecular Imaging}

The need for tools to facilitate the visualization of the different molecular processes occurring in the human body in a non-invasive, accurate way has led to the development of molecular imaging. ${ }^{1-3}$ Originally started in the field of radiopharmacology, this diagnostic discipline combines knowledge from biology, physics, chemistry and medicine.

\subsubsection{General Aspects}

In the clinic, established (non-invasive) molecular imaging techniques used nowadays include positron emission tomography (PET), single photon emission computed tomography (SPECT), magnetic resonance imaging (MRI) and several optical imaging techniques based on fluorescence, absorption and bioluminescence. ${ }^{3-5}$ For all of these modalities, an imaging agent $(I A)$ is used in order to aid the visualization of certain regions of interest (ROI) without perturbing the molecular pathways to be visualized. ${ }^{6}$ IAs used for the previously mentioned imaging modalities usually consist of radioactive, paramagnetic or fluorescent moieties, alone or in combination with e.g., antibodies or peptides to target specific cells in the body.

General requirements for these IAs, especially when it comes to in vivo applications, include high kinetic $^{7}$ and thermodynamic stability, water solubility, chemical stability at physiological $\mathrm{pH}$, long-term stability at kidney environment $(\mathrm{pH} \sim 4.5)$, and low toxicity. Other features, such as target specificity and excretion time after administration are crucial in a more advanced stage of clinical testing and once the previously mentioned criteria are met. 


\subsubsection{The Need for Different Modalities: Complementarity}

In spite of the various imaging techniques available nowadays, there is not a single technique that provides all the information needed in a single scan. Typical examples are techniques derived from nuclear medicine, such as PET and SPECT, which offer physiological information and are highly sensitive, as they allow detection of IAs in the pico-molar range (Figure 2.1). However, these modalities suffer from limited spatial resolution and, therefore, require a second technique (e.g. computed tomography -CT-) to provide anatomical detail that can be correlated to the information gathered.

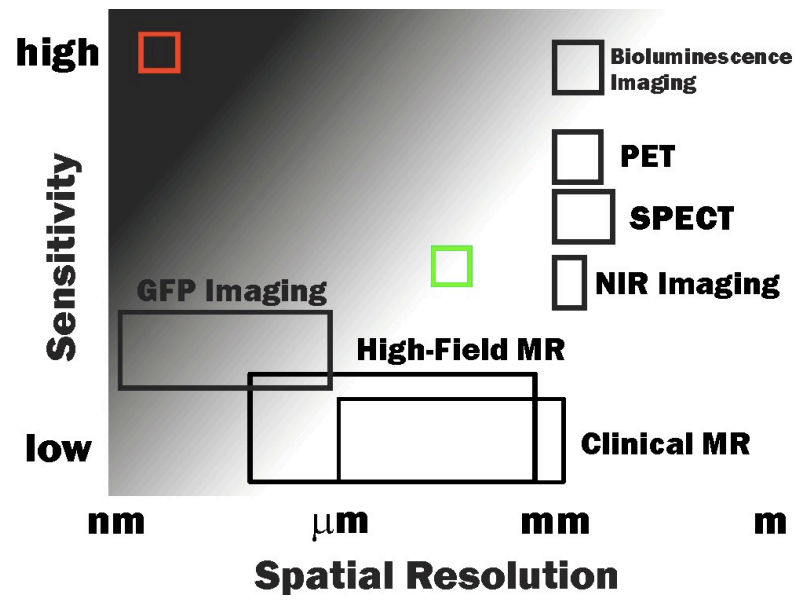

Figure 2.1. Sensitivity vs. spatial resolution of selected imaging modalities, including the position of the ideal imaging modality (red) and its real/achievable positioning (green). Adapted from. ${ }^{4}$

Alternative techniques such as MRI offer anatomic (detailed) information, soft tissue contrast and excellent spatial resolution in the sub millimeter range. Still, MRI suffers from a rather low sensitivity. On a cellular level, optical techniques based on absorption and (self)emission of light using near-infrared dyes and bioluminescent compounds (enzymatic assays) 
have become very popular due to the high sensitivity they offer. For most of these techniques, with the exception of green fluorescent protein (GFP) imaging, the limited spatial resolution remains an issue. Other drawbacks include tissue penetration depth, altered photon transport as a result of scattering and absorption phenomena, photobleaching, autofluorescence and (sometimes) the need for preparates for ex vivo analysis. ${ }^{3,4}$

Ideally, the ultimate imaging technique will offer the best spatial resolution, excellent soft tissue contrast and a very high sensitivity (Figure 2.1, red square). While with the present technical knowledge and imaging modalities available this seems unlikely, the merge of two (or more) imaging techniques appears to be the most intuitive way to reach a compromise between high spatial resolution and sensitivity (Figure 2.1, green square). In order to obtain information from different imaging techniques about a certain $\mathrm{ROI}$, the so-called multimodal agents are used: these are IAs where active moieties for different imaging modalities are combined.

\subsection{Multimodal Imaging}

Multimodal probes have become increasingly popular due to their several advantages, which include the possible visualization of biological material using different, complementary techniques. Additionally, multimodal IAs can help see "hot spots" in the operation room after localization with noninvasive routine techniques ${ }^{8}$ and are excellent labels for in vivo validation of (new) agents. There are innumerable approaches ranging from pure synthetic chemistry (in order to make probes with the different imaging techniques combined in one molecule), to the modification of fairly established IAs that can be combined, with the help of surface chemistry, 
to nanostructures/nanomaterials. These advances have led to a complete library of combinations of various imaging techniques, which will be highlighted in the next sections.

\subsubsection{The Concept of Multimodality}

In literature, terms such as multimodality probes, ${ }^{8}$ multimodal agents ${ }^{9}$ and and/or dual agents ${ }^{10}$ are used when referring to systems where various IAs are combined into one whole. In this way, detection using different imaging modalities can be achieved. Nevertheless, there is some ambiguity regarding the concept of duality, since the terms dual probe or dual agent are also used when referring to probes where little variations (e.g., by changing the lanthanide in a molecule) ${ }^{11,12}$ lead to separate IAs that can be used for different modalities.

\subsubsection{Combinations of Imaging Modalities}

Throughout the years, multimodal agents with functionalities for optical, magnetic and/or nuclear imaging techniques have been developed, studied and, in some cases, pre-clinically tested. ${ }^{8,10}$ An excellent example from the past few years regards the development of the PET/MRI combination. ${ }^{13-15}$ Synergistically, the spatial resolution issues of PET are addressed by MRI while, at the same time, the low sensitivity or MRI is overcome by the pico-molar range concentrations that can be detected using PET (Figure 2.2). ${ }^{16}$ 

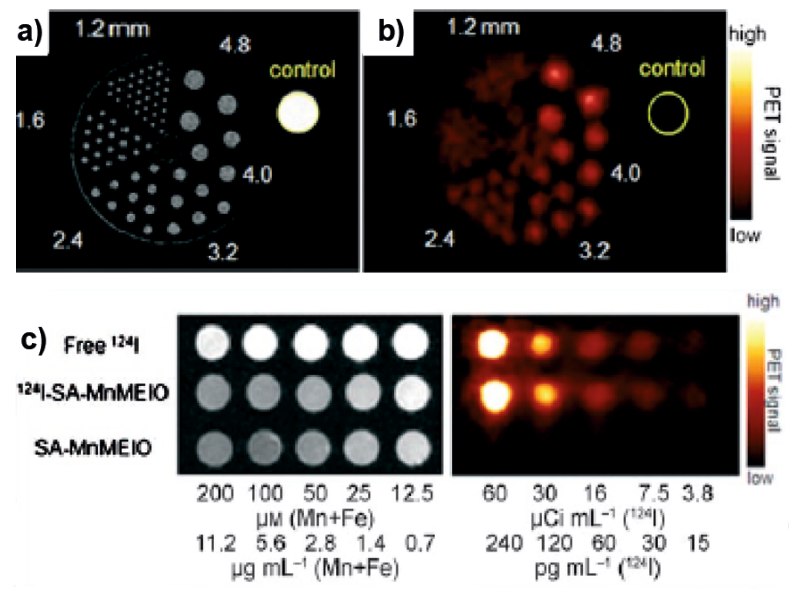

Figure 2.2. Spatial resolution of a) MRI vs. b) PET, illustrated using a Derenzo phantom containing Mn-doped magnetism-engineered iron oxide nanoparticles (MnMEIO). Yellow circle: water (control). c) Sensitivity-related information illustrated by MR (left) and PET (right) images of free ${ }^{124} \mathrm{I},{ }^{124} \mathrm{I}-\mathrm{SA}-\mathrm{MnMEIO}$ and SA-MnMEIO contrast agents at different concentrations (SA: serum albumin). Adapted with permission from. ${ }^{16}$ Copyright 2008 John Wiley \& Sons.

Another example is the combination optical/nuclear imaging, where the unlimited depth penetration of nuclear imaging is combined with the better resolution and (sometimes) higher sensitivity that optical imaging provides for superficially localized lesions..$^{10,17,18}$ In addition to this, nuclear imaging can also be used as a validation technique for (new) optical IAs. ${ }^{19,20}$ Despite the innumerable technical/chemical/biological developments related to the previously mentioned combinations, magnetic resonance/optical imaging remains by far the most studied and developed blend. $^{9}$ 


\subsection{Magnetic Resonance and Optical Imaging}

When it comes to multimodal IAs, one of the prevalent combinations is optical/MR imaging, with the latter technique remaining one of the most popular and investigated imaging modalities today. Interest in MRI arises not only from the excellent spatial resolution and soft tissue contrast it offers, but also because of its non-ionizing character that distinguishes it from nuclear imaging techniques. Additionally, MRI has been used for a broader range of applications, including blood flow, metabolic and cell density studies. ${ }^{21}$ Optical imaging, on the other hand, helps balance out the sensitivity issues related to MRI while still offering spatial resolutions in the sub-micron range and a non-ionizing character. ${ }^{22,23}$

\subsubsection{Magnetic Resonance Imaging (MRI)}

Magnetic resonance imaging (MRI) is a non-invasive technique mostly associated with medical diagnostics due to its ability to discriminate between different kinds of tissue, in 2 and 3-dimensional views, of a certain part of the human body. Unlike other commonly used imaging techniques such as PET, MRI has a non-ionizing character, making it suitable for cell research since no cell damage occurs. Typical magnetic field strengths used for medical/diagnostic imaging include $0.5,1.5$ and 3 Tesla, the latter being the most used in modern hospitals.

In the presence of a strong external magnetic field $B_{0}$, the nuclear magnetic moments of protons ( $(\mathrm{H}$, quantum spin number $\mathrm{I}=1 / 2)$ stop being randomly oriented and adopt parallel or anti-parallel orientations to the magnetic field due to energetic preferences. The population difference that arises from the adopted spin states $( \pm 1 / 2)$ in the presence of $B_{0}$ is known as the macroscopic (or bulk) magnetization $M_{0}$ (average magnetic moment, 
Figure 2.3a,b). If an applied radiofrequency ( $\mathrm{rf}$ ) pulse generates an electromagnetic field of a resonance frequency that can be absorbed by the spins (known as the resonance frequency), the position of the magnetization vector $M_{0}$ changes in space as it inclines away from the reference axis (Figure 2.3c). The phenomenon of realignment of the $M_{0}$ with the external magnetic field $B_{0}$ is known as relaxation (Figure 2.3d).

a)

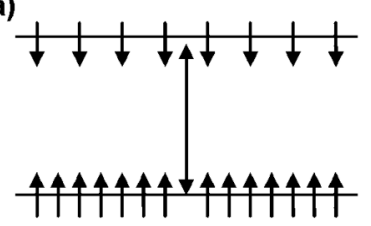

b)

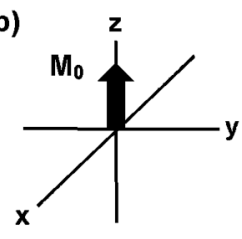

c)

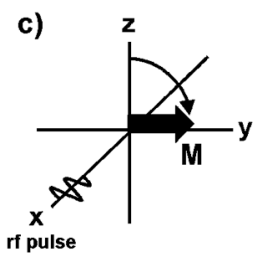

d)

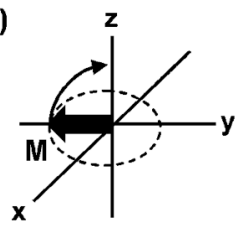

Figure 2.3. a) Population difference, that gives rise to the magnetization vector $M_{0}$, due to the possible spin states. b) Macroscopic magnetization in thermal equilibrium. c) The magnetization vector moves away from the z-axis as an rf pulse is applied. d) Precession of the magnetization vector as it realigns with the magnetic field.

The use of magnetic resonance for medical purposes was first explored in 1971, when Damadian ${ }^{24}$ reported differences in the (water) proton relaxation of both normal and cancer tissues and proposed scanning of living people. Further studies focused on the development of imaging techniques and by 1973, Paul Lauterbur ${ }^{25}$ had proven that, in the presence of a magnetic field, a relationship between location and frequency could be established. This is achieved using a spatial gradient of the static field $\mathrm{B}_{0}$, which causes linear variations of the spatial location of nuclei with their resonance frequency (Figure 2.4a). ${ }^{26}$ In this way, spatial resolution is achieved as the position of a given nucleus can be identified. Inversed Fourier Transformation of the signals, expressed as several points in a coordinate system (the so-called reciprocal space), is used to generate 
and image which shows the proton distribution in the body (real space) (Figure 2.4b).

a)

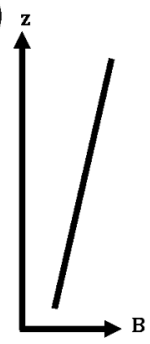

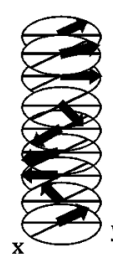

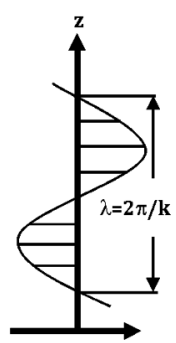

b)

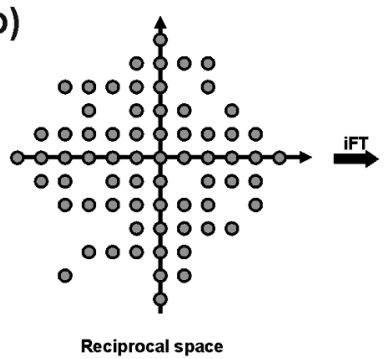

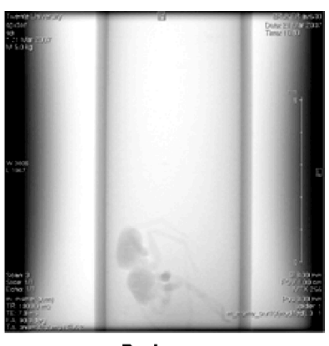

Real space

Figure 2.4. a) In the presence of a linearly space-dependent magnetic field in the zdirection (gradient), the phase of the magnetization in the $y$-direction $\left(M_{y}\right)$ changes in time. Adapted from. ${ }^{27}$ b) MR image is produced by inversed Fourier Transformation (iFT) of the reciprocal space to the real space.

\subsubsection{Proton Relaxation and Image Contrast}

After an rf pulse is applied to a sample in the presence of a magnetic field $\mathrm{B}_{0}$, the magnetization vector will tend to return to the thermal equilibrium state; a phenomenon known as relaxation. During this process, two kinds of relaxation occur:

i) spin-lattice or longitudinal relaxation ( $T_{1}$, Figure 2.5a): described as the time it takes for the magnetization vector to return to its thermal equilibrium state (relaxation in the $z$ direction). This enthalpic process, where spins simply loose energy, can take seconds, minutes, hours or even days.

ii) spin-spin or transverse relaxation ( $T_{2}$, Figure $\left.2.5 \mathrm{~b}\right)$ : described as the time it takes for the magnetization vector to dephase (relaxation in the $x y$ plane). This entropic process, where energy is swapped between spins (flip-flop process), takes usually milliseconds. 

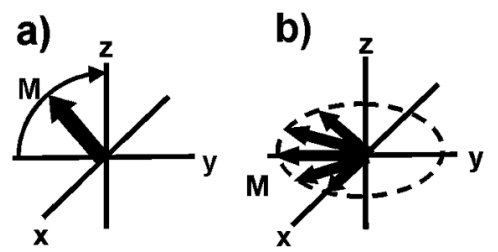

Figure 2.5. a) Spin-lattice or longitudinal relaxation $\left(T_{1}\right)$. b) Spin-spin or transverse relaxation $\left(T_{2}\right)$.

Once the magnetization vector returns to thermal equilibrium, no transverse relaxation in the xy plane can occur. For this reason, longitudinal relaxation times $T_{1}$ are always larger than transverse relaxation times $T_{2}$. The differences between these two relaxation processes form the basis for MR contrast imaging, generated by optimizing if pulses and delay times in order to obtain, for instance, $T_{1}$ - and $T_{2}$-weighted images.

While water protons of different tissues in the body show differences in their relaxation times, visualization of possible ROls can be challenging, as tissues with similar relaxation behavior might be histologically different. In such cases a contrast agent (CA) aids the image contrast by shortening $T_{1}$ and/or $T_{2}$. Shortening of the longitudinal relaxation time $T_{1}$ leads to brighter images due to the increased signal (positive contrast), while shorter transverse relaxation times $T_{2}$ lead to negative contrast (darker images) due to signal loss. Typical $T_{1}$ CAs are based on metal complexes of paramagnetic ions, ${ }^{28-32}$ being $\mathrm{Gd}^{3+}$ the most widely used, while $T_{2}$ CAs are mostly based on super-paramagnetic iron oxides (SPIOs). ${ }^{33-35}$ Other alternatives to $\mathrm{Gd}^{3+}$-based metal chelates are described elsewhere. ${ }^{32,36}$ In the next sections, $T_{1}$ relaxivity and $\mathrm{Gd}^{3+}$-based $T_{1}$ CAs will be discussed in more detail. 


\subsubsection{First Generation $T_{1}$ Contrast Agents}

The $\mathrm{Gd}^{3+}$ ion, with its seven unpaired $f$-electrons, has a totally symmetric electronic state $\left({ }^{8} S_{7 / 2}\right.$ ground state) and a high magnetic moment $(7.9 \mu \mathrm{B})$. For these reasons, $\mathrm{Gd}^{3+}$ has much longer relaxation time than other lanthanide ions. ${ }^{30,37}$ The 8 water molecules in the first coordination sphere of the $\mathrm{Gd}^{3+}$-aquo ion are responsible for the high relaxivity it offers. However, $\mathrm{Gd}^{3+}$ ions are often "clustered" using organic ligands, as this lanthanide is very toxic in its "free" form. For decades, metal complexes have been widely used as IAs/diagnostic tools, ${ }^{31}$ especially for oncologic applications. Since their introduction in the late $80 \mathrm{~s},{ }^{38}$ extracellular CAs based on $\mathrm{Gd}^{3+}$ complexes of polyamino polycarboxylate ligands ${ }^{39}$ are used in approximately one third of all the MRI exams in the world. These CA have an extracellular character since the most stable complexes, formed using negatively charged ligands, are repelled by the negatively charged head groups of the phospholipids in the membrane.

Complexes used nowadays for clinical diagnostics include $\mathrm{Gd}^{3+}$ diethylenetriaminepentaacetic acid $\left(\mathrm{Gd}^{3+}\right.$-DTPA, Magnevist $\circledast$ ) and $\mathrm{Gd}^{3+}$ 1,4,7,10-tetraazacyclododecanetetraacetic acid $\left(\mathrm{Gd}^{3+}\right.$-DOTA, Dotarem®) (Scheme 2.1, left and right, respectively). These CAs enhance the contrast of images in the regions where they are located due to a lowered longitudinal relaxation time $T_{1}$.
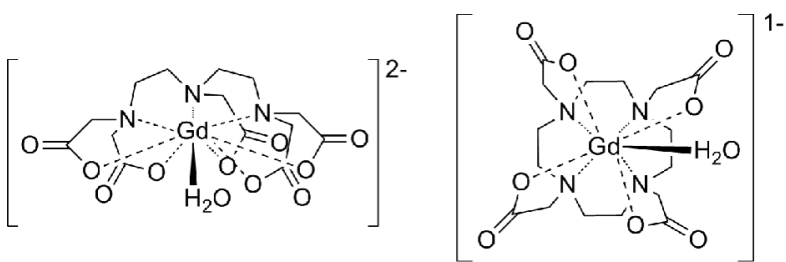

Scheme 2.1. Chemical structure of $\mathrm{Gd}^{3+}$-DTPA (left) and $\mathrm{Gd}^{3+}$-DOTA (right). 


\subsubsection{Relaxivity}

The relaxation effect observed in the presence of metal complexes is due to the interaction of the electron spins of the paramagnetic ion with the hydrogen nuclei of water. In the presence of paramagnetic species, the observed relaxation rate $\left(R_{\mathrm{i}}=1 / T_{\mathrm{i}}\right)_{\text {obs }}$ can be expressed as the contribution from the relaxation rate in: a) the absence of the paramagnetic solute, known as the diamagnetic term $\left(1 / T_{i}\right)_{d}$ and $\left.b\right)$ the presence of the paramagnetic substance $\left(1 / T_{i}\right)_{p}$, which is known to be linearly proportional to the concentration of the paramagnetic solute (Equation 2.1). ${ }^{40}$

$$
\left(\frac{1}{T_{i}}\right)_{o b s}=\left(\frac{1}{T_{i}}\right)_{d}+\left(\frac{1}{T_{i}}\right)_{p}=\left(\frac{1}{T_{i}}\right)_{d}+r_{i}[G d]
$$

The $T_{\mathrm{i}}$-shortening efficiency shown by a CA is known as relaxivity $r_{\mathrm{i}}(\mathrm{i}=1,2)$, defined as the efficacy of a $1 \mathrm{mM}$ solution of CA to increase the relaxation rate $R_{\mathrm{i}}$ of water protons. In Table 2.1, the $r_{1}$ relaxivities rates of some commercially available CA for MRI are shown.

Table 2.1. Relaxivity $\left(r_{1}\right)$ values of some clinically used $\mathrm{Gd}^{3+}$-based contrast agents for MRI, measured at $37^{\circ} \mathrm{C}$ and $1.5 \mathrm{~T}^{30}$

\begin{tabular}{|c|c|c|}
\hline Trademark & Abbreviation of the active component & $r_{1} / \mathrm{mM}^{-1} \mathrm{~s}^{-1}$ \\
\hline Dotarem $\circledast$ & $\mathrm{Gd}^{3+}-$ DOTA & 4.2 \\
\hline ProHance $®$ & $\mathrm{Gd}^{3+}-\mathrm{HP}-\mathrm{DO} 3 \mathrm{~A}$ & 4.4 \\
\hline Gadovist $\circledast$ & $\mathrm{Gd}^{3+}-\mathrm{BT}-\mathrm{DO} 3 \mathrm{~A}$ & 5.3 \\
\hline Magnevist $\AA$ & $\mathrm{Gd}^{3+}-\mathrm{DTPA}$ & 4.3 \\
\hline Omniscan $\AA$ & $\mathrm{Gd}^{3+}$-DTPA-BMA & 4.6 \\
\hline OptiMARK® & $\mathrm{Gd}^{3+}$-DTPA-BMEA & 5.2 \\
\hline MultiHance $®$ & $\mathrm{Gd}^{3+}$-BOPTA & 6.7 \\
\hline Primovist $\AA$ & $\mathrm{Gd}^{3+}$-EOB-DTPA & 7.3 \\
\hline Vasovist ${ }^{\circledR}$ & MS-325 & 19 \\
\hline
\end{tabular}


As shown in Equation 2.2, two mechanisms contribute to the paramagnetic relaxation enhancement effect: inner-sphere relaxation, resulting from the exchange of coordinated water molecules (first coordination sphere) with the bulk environment, and outer-sphere relaxation, related to water molecules in the surroundings:

$$
\left(\frac{1}{T_{i}}\right)_{p}=\left(\frac{1}{T_{i}}\right)_{\text {inner-sphere }}+\left(\frac{1}{T_{i}}\right)_{\text {outer-sphere }}
$$

Inner-Sphere Relaxation. The inner-sphere contribution to the relaxation rate is usually modeled using the Solomon-BloembergenMorgan (SBM) theory. ${ }^{29,40}$ According to this theory, two relaxation mechanisms contribute to both the longitudinal $\left(R_{1}\right)$ and transverse $\left(R_{2}\right)$ relaxation rates, namely the dipole-dipole (DD) and scalar (SC) mechanisms, generally expressed as follows:

$$
\begin{aligned}
& \frac{1}{T_{i M}}=\frac{1}{T_{i}^{D D}}+\frac{1}{T_{i}^{S C}} \quad \mathrm{i}=1,2 \\
& \frac{1}{T_{1}^{D D}}=\frac{2}{15} \frac{\gamma_{I}^{2} g^{2} \mu_{B}^{2} S(S+1)}{r^{6}}\left[\frac{3 \tau_{c 1}}{\left(1+\omega_{I}^{2} \tau_{c 1}^{2}\right)}+\frac{7 \tau_{c 2}}{\left(1+\omega_{s}^{2} \tau_{c 2}^{2}\right)}\right] \\
& \frac{1}{T_{1}^{S C}}=\frac{2}{3} S(S+1)\left(\frac{A}{\hbar}\right)^{2}\left[\frac{\tau_{e 2}}{\left(1+\omega_{s}^{2} \tau_{e 2}^{2}\right)}\right] \\
& \frac{1}{T_{2}^{D D}}=\frac{1}{15} \frac{\gamma_{I}^{2} g^{2} \mu_{B}^{2} S(S+1)}{r^{6}}\left[\frac{3 \tau_{c 1}}{\left(1+\omega_{I}^{2} \tau_{c 1}^{2}\right)}+\frac{13 \tau_{c 2}}{\left(1+\omega_{s}^{2} \tau_{c 2}^{2}\right)}+4 \tau_{c 1}\right]
\end{aligned}
$$




$$
\begin{array}{ll}
\frac{1}{T_{2}^{S C}}=\frac{1}{3} S(S+1)\left(\frac{A}{\hbar}\right)^{2}\left[\frac{\tau_{e 2}}{\left(1+\omega_{s}^{2} \tau_{e 2}^{2}\right)}+\tau_{e 1}\right] & \\
\frac{1}{\tau_{c i}}=\frac{1}{T_{i e}}+\frac{1}{\tau_{M}}+\frac{1}{\tau_{R}} & \mathrm{i}=1,2 \\
\frac{1}{\tau_{e i}}=\frac{1}{T_{i e}}+\frac{1}{\tau_{M}} & \mathrm{i}=1,2
\end{array}
$$

Where: $\quad \tau_{c i}-\tau_{e i}$ : correlation times dipole-dipole and scalar relaxation $\gamma$ : nuclear gyromagnetic ratio

$g$ : electron $g$ factor

S: spin quantum number

$\mu_{\mathrm{B}}$ : Bohr magneton

$\omega_{\mathrm{s}}$ and $\omega_{\mathrm{l}}$ : electron and nuclear Larmor precession frequencies, respectively $(\omega=\gamma B$, where $B$ is the magnetic field)

$r$ : electron spin-solvent nuclear spin distance

$A / \hbar$ : electron-nuclear hyperfine coupling constant

$\tau_{\mathrm{R}}$ : molecular rotational coordination time or tumbling rate

$\tau_{\mathrm{m}}$ : water residence time

$T_{\mathrm{ie}}$ : electronic longitudinal $(\mathrm{i}=1)$ and transverse $(\mathrm{i}=2)$

relaxation times for the metal ion

The SBM equations above (Equation 2.3-2.9) show that relaxation rates (interpreted in terms of zero-field splitting interactions -ZFS-) are field dependent, since the gyromagnetic ratio $\gamma$ depends on the magnitude of 
this field $(\omega=\gamma B)$. Therefore, the maximum achievable relaxivity decreases as the strength of the magnetic field increases. In such cases, the outersphere relaxation mechanisms become more important (see next section). Extra details regarding the SBM theory can be found elsewhere..$^{28,38}$

Since relaxed water protons exchange rapidly with molecules from the surroundings, relaxation is dictated by the number of water molecules coordinated to the paramagnetic center $(q$, known as the hydration number), their residence time $\left(\tau_{m}\right)$, the rotational correlation time or tumbling rate $\left(\tau_{R}\right)$ and the diffusion correlation time $\left(\tau_{D}\right.$, Figure 2.6$)$.

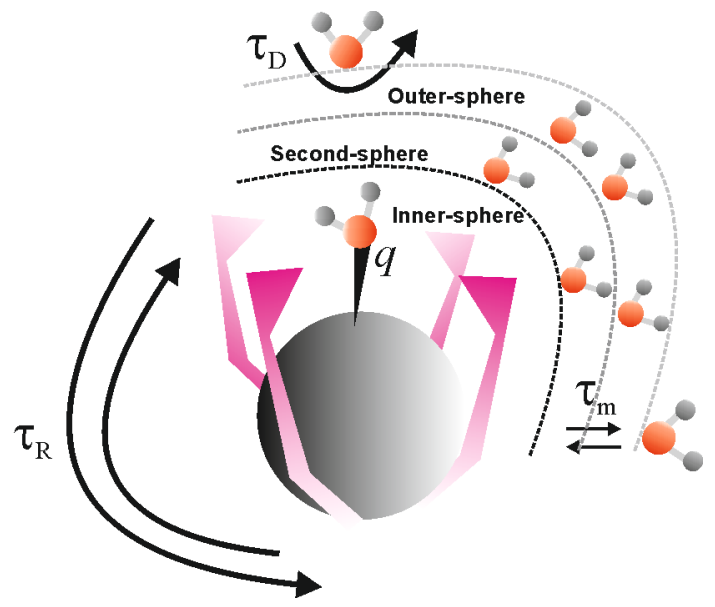

Figure 2.6. Inner- and outer-sphere parameters that dictate the relaxivity of $\mathrm{Gd}^{3+}$-based CAs. Adapted from. ${ }^{28,30}$

Optimization of $\tau_{\mathrm{m}}$ is necessary as the time a coordination site on the metal ion is occupied by a water molecule dictates the relaxation efficiency of a $\mathrm{CA}$. The tumbling rate $\tau_{\mathrm{R}}$ (which becomes slower when the size/volume of CAs increases) also plays a key role as lowered global rotational motion of CAs leads to higher relaxivity values. Intuitively, the relaxivity is strongly affected by the number of bound water molecules per metal ion. However, the ability to increase $q$ is limited by safety issues because, typically, the 
more coordination sites available for water ligation, the lower the thermodynamic stability of the complex. Second generation CAs based on hydroxypyridinone ligands, ${ }^{41-47}$ which will not be discussed in this literature review, address this issue with rather respectable results.

Outer-Sphere Relaxation. The paramagnetic relaxation effect is not only limited to solvent molecules in the first coordination sphere, as some degree of relaxivity has been observed in complexes with $q=0 .{ }^{48}$ When the solvent molecules diffuse to the (magnetized) "external" solute sphere, their protons experience variations in the local magnetic field..$^{49}$ While the exact outer-sphere contributions to the relaxivity are not well understood yet, it is known that the diffusion correlation time $\tau_{D}$ of water molecules, ${ }^{29}$ as well as relaxation by dipolar mechanisms of hydrogen bonded water molecules in the second-sphere, ${ }^{50,51}$ play an important role.

Relaxivity at High Magnetic Fields. Typical magnetic fields used for clinical imaging include $0.5,1.5$ and $3 \mathrm{~T}$. At these field strengths, an optimal balance between fast water exchange and enhanced tumbling rate of the complex is desired, together with a higher contribution from the second coordination sphere water molecules. ${ }^{52}$ As higher magnetic fields are implemented, the maximum relaxivity achievable decreases and tends to depend on careful "fine-tuning" of $\tau_{m}$ and $\tau_{R}{ }^{29}$ Yet, this decrease in the relaxivity achievable is compensated by the higher sensitivity (due to greater signal-to-noise ratio) and increased spatial resolution that can be attained using high-field MRI instrumentation. 


\subsubsection{The Sensitivity Gap: Increasing the MRI Signal}

One of the major challenges when developing new CAs for MRI is dealing with the inherent low sensitivity and the need for high concentrations of CAs: "in order to induce observable contrast in a robust clinical exam, a relaxation rate change of at least about $0.5 \mathrm{~s}^{-1}$ is required. For extracellular commercial contrast agents with a relaxivity of $\sim 4 \mathrm{mM}^{-1} \mathrm{~s}^{-1}$, this means a concentration of $\sim 125 \mu \mathrm{M}$. For targeted imaging and assuming a 1:1 binding stoichiometry $\left(G d^{3+}\right.$ :target molecule), this would require a biological target present with a concentration at least $125 \mu M^{\prime \prime 53}$

Through the years, efforts have focused on the design of new ligands to form metal complexes with optimized relaxivity-related parameters such as the hydration number $q^{41-44}$ the water residence time $\tau_{m}^{54-60}$ and the molecular tumbling rate $\tau_{\mathrm{R}}$. Slower global rotational motion, and therefore higher relaxivity, has successfully been achieved by increasing the molecular weight of the metal complexes. This has been done by means of covalent and non-covalent attachment to e.g., proteins, ${ }^{61,62}$ peptides, ${ }^{63}$ human serum albumin ${ }^{64}$ and ATP. ${ }^{65}$ An alternative strategy followed involves the attachment of $\mathrm{Gd}^{3+}$ complexes to macromolecules ${ }^{66}$ in order to: i) decrease the tumbling rate and ii) increase the local paramagnetic moiety concentration..$^{67,68}$ There are numerous examples in the literature illustrating the improved relaxivity properties that can be achieved by incorporating $\mathrm{Gd}^{3+}$ complexes to, e.g., dendrimers, ${ }^{69-75}$ proteins (polypeptides), ${ }^{61,76-79}$ polymers, ${ }^{80,81}$ proteins cages such as wild-type cowpea mosaic virus (CPMV), ${ }^{82}$ HSP capsids, ${ }^{83}$ cowpea chlorotic mottle virus (CCMV) capsids $^{84}$ and MS2 viral capsids, ${ }^{85-87}$ cyclic oligosaccharides, ${ }^{88}$ carbohydrates $^{89}$ peptides, $^{90-92}$ metallostars $^{93,94}$ and supramolecular aggregates such as micelles and liposomes. ${ }^{95-100}$ 
A more recent strategy uses advances in the field of nanotechnology in order to decrease the molecular motion of complexes (by increasing their volume) and boost the local $\mathrm{Gd}^{3+}$ concentration. Most of these approaches, based on surface modification of diverse nanomaterials, not only show improved relaxivities but also require less synthetic input. In addition to this, surface modification opens up the door to the facile incorporation of other moieties for, e.g., biocompatibility and targeting. Examples of such "paramagnetic nanosystems" include quantum dots, ${ }^{101-}$ 104 nanodiamonds, ${ }^{105}$ (silica) nanoparticles, ${ }^{106-108}$ mesoporous silica nanospheres, ${ }^{109}$ nanoassembled capsules of polymer aggregates inside silica shells, ${ }^{110}$ carbon nanotubes, ${ }^{111-113}$ gold nanoparticles ${ }^{114-118}$ and gold nanorods. ${ }^{119,120}$

\subsubsection{Optical Imaging}

Whereas standard optical techniques are based on interactions of photons with matter, these elementary particles can also interact with tissue. Optical imaging of biological tissues relies on the fact that photons can interact with certain tissue components thereby affecting the number of transmitted photons that pass through irradiated ROls. This imaging modality is usually associated with traditional microscopy techniques which are known to be limited by the mean free path (MFP) of a photon, which is around $100 \mu \mathrm{m}$ in tissue. ${ }^{121}$ Contrary to nuclear or magnetic resonance imaging techniques, where detection is mainly performed in vivo, some optical imaging techniques involve in vitro as well as ex vivo experimentation, where thin tissue sections are usually required. More recently, new optical techniques based on image fusion, multichannel imaging, fiber-optic approaches and tomographic reconstruction are used to generate images in vivo. ${ }^{22}$ 


\subsubsection{Optical Imaging Techniques}

Typical optical imaging techniques currently used in the clinic are based on: i) absorption (absorption of photon energy by tissue) and ii) scattering phenomena (light deviated from its original path) that can occur in three spectral regions, ultraviolet (UV, < $450 \mathrm{~nm}$ ), visible (Vis, 450-650 nm) and near-infrared (NIR, 650-1000 nm). In the next sections, selected examples of optical absorption-based techniques, such as bioluminescence and fluorescence imaging, will be discussed. State-of-the-art optical imaging modalities, e.g. based on protein-protein interactions, will not be discussed here. ${ }^{22,121}$

Bioluminescence Imaging (BLI). Biological activity can be tracked in vivo using enzymes that form light emitting molecules (bioluminescence imaging). Enzymes such as luciferases are known for using energy and oxygen in order to oxidize luciferin, thereby leading to the self-emission of visible light (yellow to green). Emission without the need for external light sources for excitation is particularly interesting, as biological tissues are known to absorb and emit light in the visible range. Using firefly luciferase (FL), non-invasive imaging of gene expression in mice has successfully been achieved (Figure 2.7). ${ }^{122}$ However, the need for detecting devices with sufficient sensitivity remains one of the major issues of this imaging modality. Other examples illustrating the successful implementation of BLI and new developments are described in reviews on optical imaging. ${ }^{3,5,123-}$ 125 


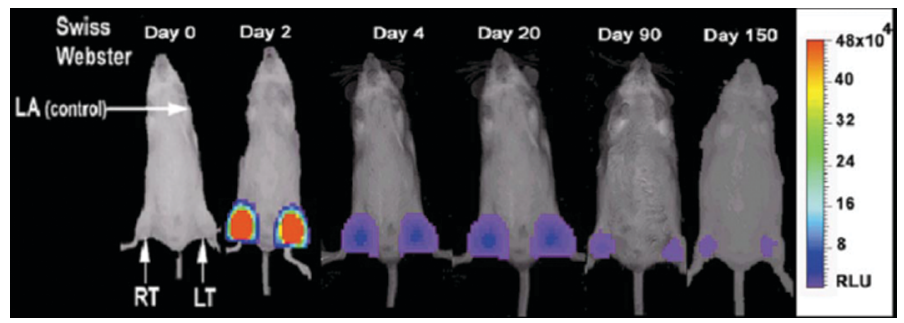

Figure 2.7. Cooled charge-coupled device (CCD) images on a Swiss Webster mouse showing the magnitude and duration of firefly luciferase $(F L)$ gene expression injected. Mice were injected with E1-deleted adenovirus expressing FL driven by a cytomegalovirus promoter (Ad-CMV-FL). Control virus yielded only background signals (55 $\pm 10 \mathrm{RLU} / \mathrm{min})$. Adapted with permission from. ${ }^{122}$ Copyright 2001 Nature Publishing group.

Fluorescence Imaging. Optical microscopy, using fluorescent molecules such as porphyrins and fluorescein for staining, has been widely used since the 1920s for in vitro applications. ${ }^{22}$ These "fluorescent agents" are used in order to alter the absorption, emission or scattering properties of the tissue where it accumulates. In this way, contrast enhancement is achieved. Taking advantage of the technological progress in microscopy and the development of fiber-optic approaches and new imaging probes, the use of fluorescent dyes has been extended to ex vivo and in vivo applications. Current techniques used include, amongst others, optical projection tomography (OPT), diffuse optical tomography (DOT), NIR fluorescence and green fluorescent protein (GFP) imaging., ${ }^{4,22,121}$ In particular, organic dyes and inorganic nanocrystals as IAs for imaging in the NIR region have been extensively studied since scattering is known to be weak in this spectral region as NIR photons travel through tissue much more efficiently. ${ }^{126}$ In the next sections, a selection of NIR IAs will be briefly discussed. 


\subsubsection{Fluorescence Imaging Agents}

Organic Dyes. Typically, organic dyes with absorption and emission properties in the NIR region can be classified in cyanine dyes, tetrapyrroles or lanthanide chelates. Water-soluble cyanine dyes (Scheme 2.2a) are very popular due to their high molar extinction coefficients $\left(>150,000 \mathrm{M}^{-1} \mathrm{~cm}^{-1}\right)$, good fluorescence quantum yields (up to 50\%), ${ }^{127,128}$ commercial availability and their ability to be further functionalized. In this way, these IAs can be linked to virtually any probe, e.g., peptides for targeting purposes (Scheme 2.2b). Other compounds used for NIR detection include tetrapyrrole-based dyes such as porphyrins, chlorins and phthalocyanines (Scheme 2.2c). In addition to the above mentioned spectroscopic properties, the latter dyes are also known for their use as photosensitizing agents in photodynamic therapy (PDT). ${ }^{127}$
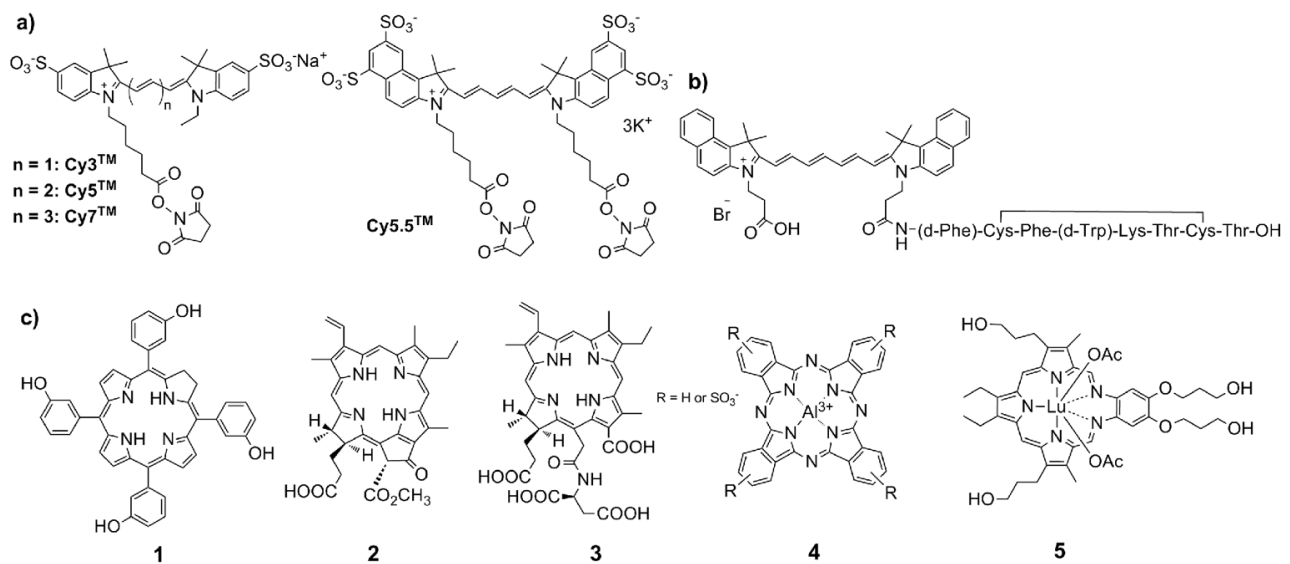

Scheme 2.2. Chemical structures of selected: a) commercially available carbocyanine dyes. b) Peptide-linked (octreotate) Cypate 9 dye. c) Chlorins $(\mathbf{1 , 2 , 3 )}$, phthalocyanines (4) and porphyrins (5). Adapted from. ${ }^{127}$

Lanthanide-based complexes are not limited to biomedical applications involving paramagnetic properties (for MRI), but can also be used for 
alternative detection modalities such as fluorescence. ${ }^{11,129}$ While europium $\left(\mathrm{Eu}^{3+}\right)$ and terbium $\left(\mathrm{Tb}^{3+}\right)^{12,130}$ are poor MRI CAs compared to $\mathrm{Gd}^{3+}$, they are highly attractive as fluorescent probes due to their long fluorescence lifetimes and large Stoke shifts, which allows detection with high selectivity/sensitivity and limited background interference. For lanthanide ions, electronic transitions in the $4 \mathrm{f}^{\mathrm{n}}$ configuration are Laporte forbidden, whereby electronic $f \rightarrow f$ transitions are forbidden for molecules exhibiting a center of symmetry. Within the lanthanide series, $\mathrm{Eu}^{3+}$ and $\mathrm{Tb}^{3+}$ are high emitters and have excited state lifetimes in the 100-3000 $\mu$ s range. However, parity-forbidden $4 \mathrm{f}-4 \mathrm{f}$ absorptions (with very low molar absorption coefficients) are observed as the $4 f$ orbitals are shielded by the filled $5 p^{6} 6 s^{2}$ sub-shells. ${ }^{131}$ Therefore, the luminescence of lanthanide ions alone is weak and requires sensitization. Usually, carefully chosen "antennae or sensitizers" are integrated in the chelating ligand in order to absorb light and promote transitions into the excited states of the coordinated metal ion (Figure 2.8a). ${ }^{131}$ However, sensitization using antennae is a complex process where the energy gap between the lowest ligand triplet state and the lanthanide emitting level needs to be adjusted (Figure 2.8b). Typical examples of sensitizers used for energy transfer include aromatic rings, ${ }^{130,132}$ pyrene, ${ }^{133}$ azathioxanthone ${ }^{134}$ and triazolophthalazine ${ }^{135}$ or transition metals such as rhenium, ${ }^{136}$ chromium ${ }^{137}$ and ruthenium. ${ }^{138}$ 
a)

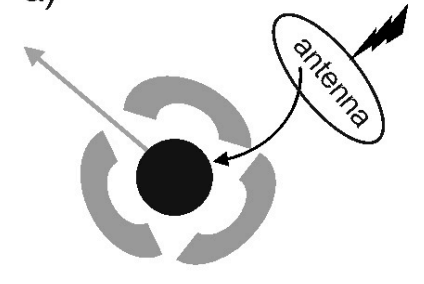

b)

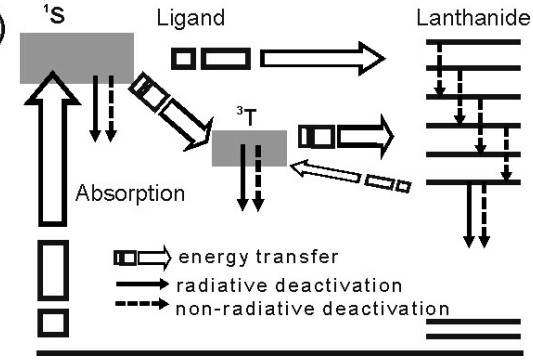

Figure 2.8. a) Sensitization using antennae. b) Diagram showing the main transition states during sensitization of lanthanide luminescence using ligands. Adapted from. ${ }^{131}$

$\mathrm{Eu}^{3+}$ and $\mathrm{Tb}^{3+}$ complexes with various sensitizers have been studied for years $^{139}$ and their potential for imaging applications has been extensively described. ${ }^{139-141}$ More recently, $\mathrm{Nd}^{3+}, \mathrm{Er}^{3+}$ and $\mathrm{Yb}^{3+}$ have become highly attractive since their emission occurs in the NIR region. ${ }^{142-144}$ Nevertheless, these metal ions also exhibit some drawbacks, including their short lifetimes (ns to $\mu \mathrm{s}$, that limit time-resolved detection) and the low sensitization due to the small energy gap between the excited and the ground-state levels.

Inorganic Fluorescent (Semiconductor) Nanocrystals. Semiconductor nanocrystals or quantum dots (QDs) consist of an inorganic core and a metal shell (with an organic outer layer) and are known for their high quantum yield, resistance to photobleaching and tunable emission wavelengths. ${ }^{145}$ Furthermore, ligand exchange of the organic outer layer or (possible) hydrophobic interactions with other molecules provide versatility to these nanoparticles. While their potential use as NIR fluorophores for in vivo optical imaging has been explored, ${ }^{146}$ the toxicity of these nanocrystals remains an issue.

Other nanocrystals with potential for optical imaging are silver nanoclusters. Consisting of a limited number of silver atoms $(n \sim 2-10)$, 
nanoclusters are known to be fluorescent ${ }^{147-150}$ and show differences in emission spectra when differing in number by one single atom. ${ }^{151,152}$ The potential of nanoclusters as IAs for optical imaging is evidenced by their successful application as single-molecule fluorophores ${ }^{153}$ and cell staining labels. ${ }^{154-156}$

\subsection{Strategies for Magnetic Resonance/Optical Multimodal Imaging Agents}

The potential of multimodal agents for applications in magnetic resonance and optical imaging is evidenced by the dramatic increase in literature during the past decades. ${ }^{8,10}$ Generally speaking, multimodal IAs can be divided in three major groups: small molecule IAS, macromolecular IAs and nanostructures. In the next sections, some representative examples of IAs for these two imaging modalities will be presented and shortly discussed.

\subsubsection{Small Molecule Imaging Agents}

Mononuclear Dual Imaging Agents. Owing to the small differences in radii between lanthanides, small molecular templates offer great versatility as they can complex either $\mathrm{Gd}^{3+}$ (for MRI) or $\mathrm{Eu}^{3+} / \mathrm{Tb}^{3+} / \mathrm{Yb}^{3+}$ (for optical imaging). Additionally, these complexes are expected to show the same biological behavior. This concept, often referred to as duality, has been proved using various chelators. ${ }^{157}$ For instance, Picard et al. ${ }^{12}$ used ligands derived from an N,C-pyrazolylpyridine moiety and iminodiacetate units (1, Scheme 2.3) to make lanthanide complexes with high thermodynamic stability. The luminescence and relaxometric properties of the $\mathrm{Tb}^{3+}$ and $\mathrm{Gd}^{3+}$ chelates, respectively, were investigated in aqueous solutions. The 
$\mathrm{Tb}^{3+}$ chelate is strongly luminescent, with a remarkable lifetime and quantum yield ( $\tau=1.82 \mathrm{~ms}$ and $\Phi=0.42$, respectively). Moreover, the longitudinal proton relaxivity of the $\mathrm{Gd}^{3+}$ chelate was found to be comparable to that of the clinically used $\mathrm{Gd}^{3+}$-DTPA. Cyclic polyaminocarboxylates, such as 10-(2-hydroxypropyl)-1,4,7tetraazacyclododecane-1,4,7-triacetic acid (HP-DO3A 2, Scheme 2.3), have also been studied as dual MR/optical imaging IAs. In order to do so, Geninatti Crich et al. ${ }^{11}$ incubated endothelial progenitor cells (EPCs) with $\mathrm{Gd}^{3+}$ and $\mathrm{Eu}^{3+}-\mathrm{HP}-\mathrm{DO} 3 \mathrm{~A}$ complexes. The $\mathrm{Gd}^{3+}$ chelates were used as $T_{1^{-}}$ CAs for MRI visualization while the $\mathrm{Eu}^{3+}$ chelates acted as reporters/"validation" agents for fluorescence microscopy.

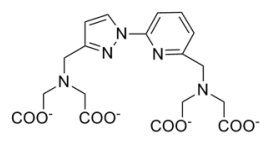

1

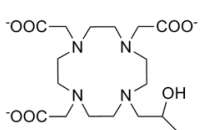

2

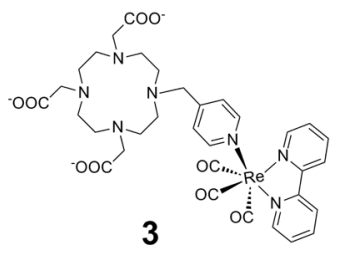

3

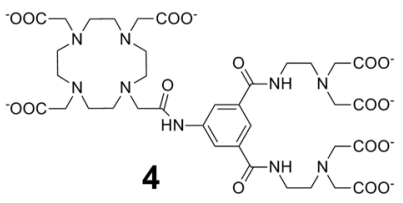

Scheme 2.3. Chemical structures of: polyaminocarboxylate ligand derived from N,Cpyrazolylpyridine (1), ${ }^{12}$ HP-DO3A ligand (2), ${ }^{11}$ methyl pyridine DOTA derivative with bipyridine rhenium complex $(\mathbf{3})^{136}$ and DOTA/5A-PADDTA ligand (4). ${ }^{158}$

Heteronuclear Dual Imaging Agents. Another example of dual IAs is heteronuclear complexes, where two metal ions are integrated in the same molecule. For this purpose, Koullourou et al. ${ }^{136}$ used a methyl pyridine DOTA derivative for lanthanide complexation and a bipyridine rhenium chromophore for sensitization (3, Scheme 2.3). While the $\mathrm{Yb}^{3+}$ complex showed sensitized emission with long luminescence lifetimes, interesting relaxivity properties at high magnetic fields were associated to the $\mathrm{Gd}^{3+}$ complex (ionic $r_{1}=8.6 \mathrm{mM}^{-1} \mathrm{~s}^{-1}$ at $500 \mathrm{MHz}$ ). 
Heteronuclear Multimodal Imaging Agents. Heteronuclear complexes, where two chelating moieties are combined into one molecule, were proposed by Mamedov et al. ${ }^{158}$ Consisting of a cyclic DOTA and the acyclic 5-aminoisophthalamide diethylenediamine tetraacid ligand (5APADDTA, 4, Scheme 2.3), this bis-chelate was found to form stable complexes with $\mathrm{Eu}^{3+}$ and $\mathrm{Gd}^{3+}$, exhibiting luminescence and relaxometric properties, respectively. Complexation of the acyclic chelator was confirmed by ${ }^{1} \mathrm{H}$ NMR spectroscopy, where the spectrum of the ligand, its $\mathrm{Eu}^{3+}$ complex, and that containing both $\mathrm{Eu}^{3+}$ and $\mathrm{Tb}^{3+}$ confirmed the existence of the monometallic and bimetallic species. While this ligand is an impressive example of the potential use of heteronuclear complexes as multimodal agents, controlled integration of two different lanthanides in one molecule remains a difficult task.

Targeting Dual and Multimodal Imaging Agents. A more "targeted" approach to new, small molecule imaging probes for multimodality purposes has been proposed by Mindt et al. ${ }^{159}$ Using the $\mathrm{Cu}(\mathrm{I})$-catalyzed cycloaddition of terminal alkynes and azides, a "clickable" derivative of folic acid (FA) was prepared. Starting from the $\gamma$-azido-FA precursor (5, Scheme 2.4), imaging probes for NIR fluorescence (using a Cy5.5 NIR dye) and MR imaging (using a $\mathrm{Gd}^{3+}$-DOTA derivative) were successfully synthesized. Detailed spectroscopic characterization and in vitro studies showed the expected properties and the affinity of the FA-derivative CAs towards the FA receptor. Moreover, these probes are suitable for other imaging techniques since the DOTA-based ligand can be used for complexation with ${ }^{67} \mathrm{Ga},{ }^{111} \mathrm{In},{ }^{177} \mathrm{Lu}$ (tracer for SPECT) and the $\gamma$-azido-FA precursor can be clicked to ${ }^{18} \mathrm{~F}$ (tracer for PET) or ${ }^{19} \mathrm{~F}$ (for ${ }^{19} \mathrm{~F} \mathrm{MRI}$ ) derivatives. Other targeted CAs include trifunctional macrocyclic ligands 
with two phosphonic acid pendant arms for chelation of $\mathrm{Gd}^{3+} / \mathrm{Eu}^{3+}$ and a quinoline chromophore that acts as an antenna (6, Scheme 2.4). Manning et al. ${ }^{160}$ coupled this CA to 1-(2-chlorophenyl)isoquinoline-3-carboxylic acid, known to target peripheral benzodiazepine receptors (PBR). The $\mathrm{Eu}^{3+}$ complex showed visibly bright fluorescence and the $\mathrm{Gd}^{3+}$ complex had a relaxivity comparable to that of $\mathrm{Gd}^{3+}$-DTPA.

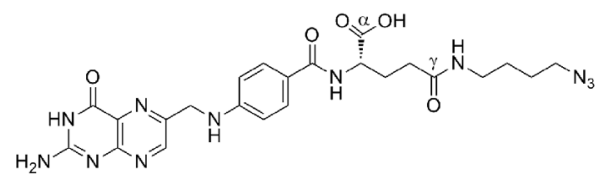

5

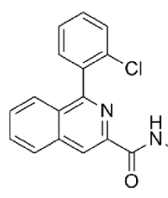

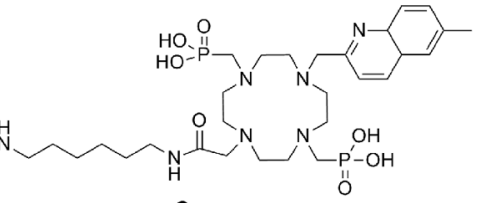

6

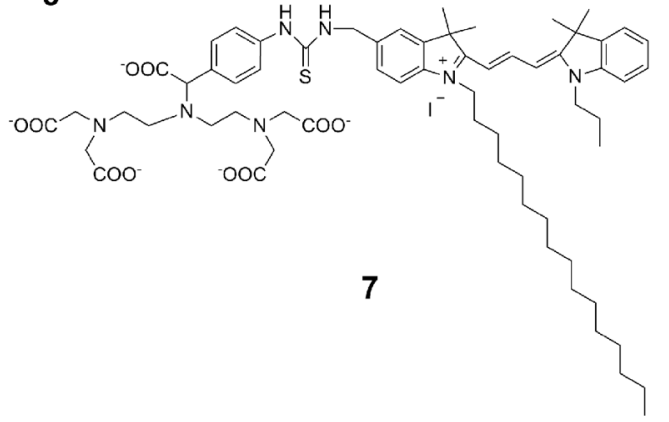

Scheme 2.4. Chemical structures of: folate- $\gamma$-(4-azido)-butane amide ${ }^{159}(5)$, trifunctional macrocyclic ligand ${ }^{160}(6)$ and carbocyanine dye with integrated DTPA (7). ${ }^{161}$

Li et al. ${ }^{161}$ have used a carbocyanine dye and a $\mathrm{Gd}^{3+}$-DTPA chelate with integrated C16 alkyl tails for labeling of low-density lipoprotein receptors (LDLs) via intercalation into their phospholipid monolayer (7, Scheme 2.4). This multimodal IA not only showed a 7 -fold increase in ionic $r_{1}$ relaxivity than $\mathrm{Gd}^{3+}$-DTPA (which allowed in vivo MRI detection of LDL receptors in liver and tumor), but it was successfully observed in vitro by confocal fluorescence microscopy. 


\subsubsection{Macromolecular Imaging Agents}

The synthetic approaches proposed for small molecule IAs have certainly led to versatile probes with improved properties. Still, these IAs are limited by their lack of selectivity and usually require the integration of targeting moieties and, therefore, longer synthetic routes. In contrast, macromolecules are an interesting alternative to achieve local high concentrations whilst still being able to integrate different imaging/targeting moieties. ${ }^{162}$ In the next sections, representative examples of the various macromolecules currently used for multimodal purposes, as well as the results achieved so far, will be highlighted.

Dendrimers. One of the first macromolecules explored for multimodal purposes were dendrimers. ${ }^{163}$ These repeated branch molecules have been used extensively to conjugate, complex or encapsulate IAs, targeting moieties and therapeutic drugs. ${ }^{164}$ Although synthetically demanding, dendrimers are chemically interesting since their surface is an excellent platform for chemical (covalent) modification and their interior is suitable for host-guest chemistry.

Considering the higher sensitivity of optical imaging when compared to $\mathrm{MRI}$, dendrimers are a fascinating platform to reach a high MRI CA ratio per optical imaging unit. For this purpose, Talanov et al. ${ }^{165}$ developed a generation 6 polyamidoamine (PAMAM) dendrimer functionalized with 2(4-isothiocyanatobenzyl)-6-methyl-diethylenetriaminepentaacetic acid (1B4M-DTPA) ligands and a Cy5.5 dye. With this nanoprobe, sentinel lymph nodes in mice were visualized in vivo using both imaging modalities (Figure 2.9). While an increase in the NIR dye content resulted in partial quenching, its quantum yield was not affected by the presence of $\mathrm{Gd}^{3+}$. 


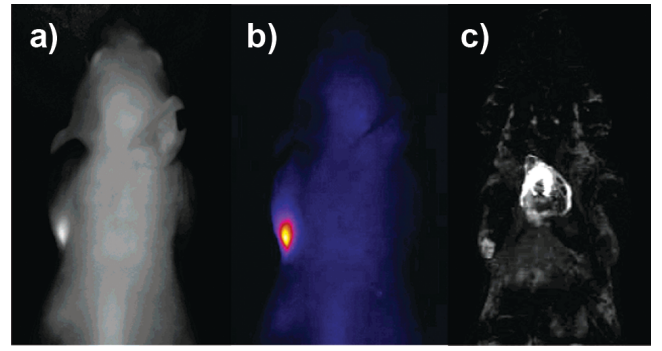

Figure 2.9. a) Optical image obtained under simultaneous white light and filtered (615-665 $\mathrm{nm}$ ) excitation light. Fluorescence emission was detected in the axillary lymph node of the mouse with the emission filter set to $720 \mathrm{~nm}$. b) Image from a) shown in false color. c) Maximum intensity projection calculated from a $3 \mathrm{D}$ spoiled gradient echo MRI, illustrating the draining lymphatics and axillary lymph node depicted in b). Adapted with permission from. ${ }^{163}$ Copyright 1999 American Chemical Society.

Macromolecules offer a distinct advantage since they can be functionalized with targeting units in order to induce selective uptake. One example of such probes was reported by Xu et al., ${ }^{166}$ in which dendrimerbased templates were functionalized with avidin-biotin and used as targeted dual-reporters. By reducing the disulfide bond of the core of $\mathrm{Gd}^{3+}$-1B4M-DTPA chelated generation 2 PAMAM dendrimers, followed by subsequent attachment to a maleimide-functionalized biotin, biotinylated dendrimer-based MRI CAs were obtained. In this work, up to 4 copies of these dendron-like structures were successfully immobilized to rhodamine green labeled avidin. In this way, mice bearing ovarian cancer tumors were efficiently targeted and visualized by both MR and optical imaging. Another targeting-integrated approach was proposed by Boswell et al., ${ }^{167}$ whereby generation 3 PAMAM dendrimers were covalently adorned with RGD-cyclopeptides, Alexa Fluor 594 dyes and Gd $^{3+}-1 B 4 M-D T P A$ complexes (Figure 2.10). In vitro studies demonstrated the capability of these multimodal dendrimers to act as IA for both MR and optical imaging. However, while fluorescence microscopy of the RGD peptide-bearing 
dendrimer with empty 1B4M-DTPA ligands showed that selective binding of $\alpha_{v} \beta_{3}$-expressing cells occurred, a decrease in selectivity was observed after complexation with $\mathrm{Gd}^{3+}$. The same effect was observed in in vivo studies, as optical fluorescence imaging failed to show appreciable tumor uptake.

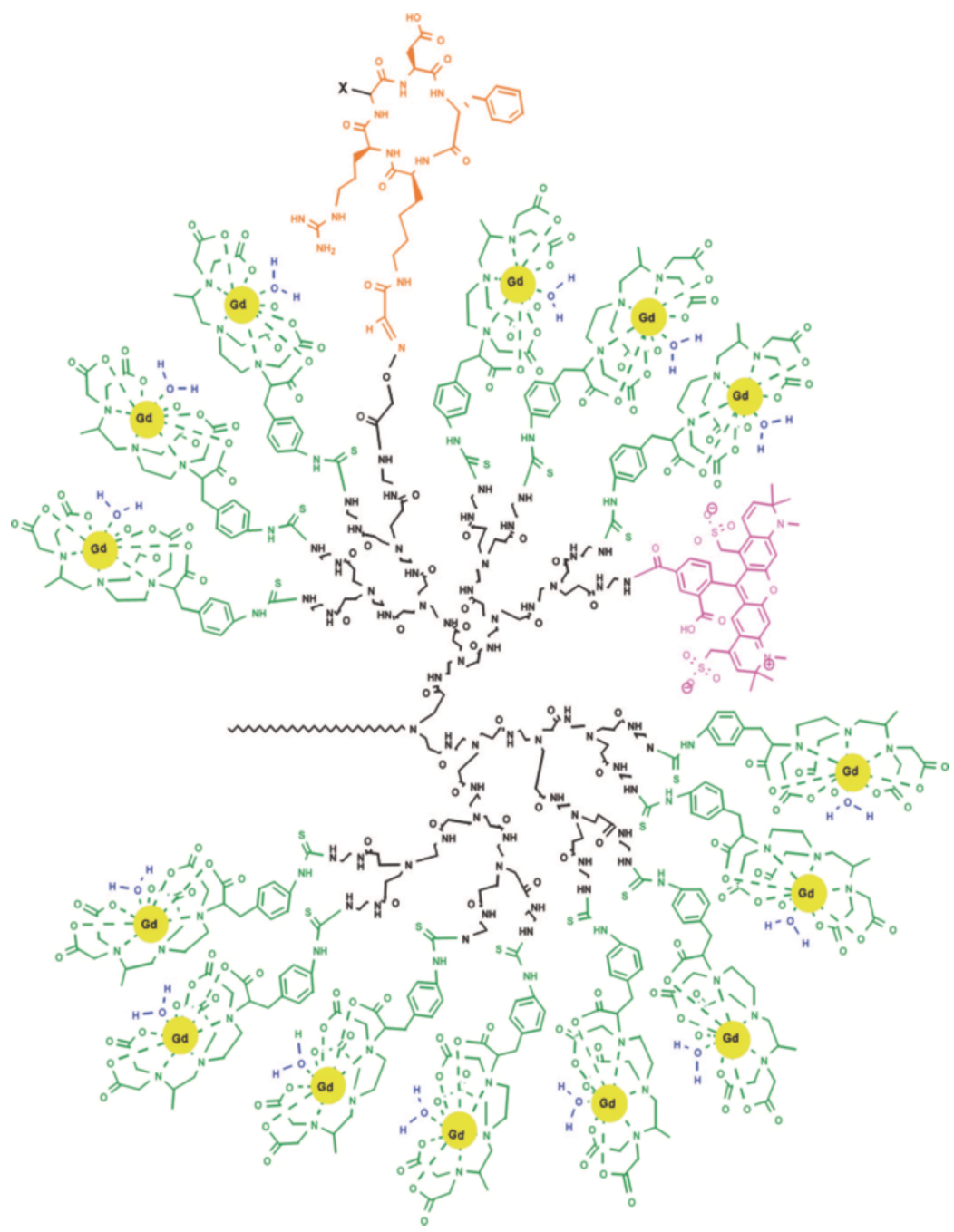

Figure 2.10. Structural representation (half-section) of the modified PAMAM dendrimer. The dendrimer core is shown in black, the $\alpha_{v} \beta_{3}$-targeting peptide (c(RGDfK)) in orange, the 1B4M-DTPA ligand in green and the Alexa Fluor 594 dye in pink. Reprinted with permission from. ${ }^{167}$ Copyright 2008 American Chemical Society. 
Despite several examples of multimodal dendrimers in literature, the use of these macromolecules for biomedical applications is limited by their large size, which often leads to rapid renal excretion even if targeting agents are used. Nevertheless, these macromolecules have successfully been used as IAs for imaging of the lymphatic system. ${ }^{168}$

Supramolecular Aggregates. Another interesting type of macromolecules proposed for multimodal purposes are supramolecular aggregates such as micelles and liposomes. These lipid-based "nanoparticles" are known to lead to higher relaxivities due to decreased molecular motion of the monomer units and are excellent scaffolds for higher local $\mathrm{Gd}^{3+}$ concentrations. ${ }^{169}$ Mulder et al. ${ }^{170}$ used PEGylated multimodal liposomes for dual detection of the expression levels of molecular markers on human umbilical vein endothelial cells (HUVEC). The paramagnetic PEGylated liposomes consisted of $\mathrm{Gd}^{3+}$-DTPAbis(stearylamide) (Gd ${ }^{3+}$-DTPA-BSA), 1,2-distearoyl-sn-glycero-3phosphocholine (DSPC), cholesterol (Chol), 1,2-dipalmitoyl-sn-glycero-3phosphoethanolamine-N-(lissamine rhodamine B sulfonyl) (rhodamine-PE) and 1,2-distearoyl-sn-glycero-3-phosphoethanolamine-N[methoxy(poly(ethylene glycol))-2000] (PEG-DSPE), with antibodies coupled to the distal end of the PEG-chains (Figure 2.11a). While in vitro targeting of HUVEC was shown by fluorescence microscopy (Figure 2.11b), relaxometric studies revealed a higher ionic $r_{1}$ relaxivity for the $\mathrm{Gd}^{3+}$-DTPA-BSA when compared to $\mathrm{Gd}^{3+}$-DTPA, especially at higher temperatures (Figure 2.11c). 

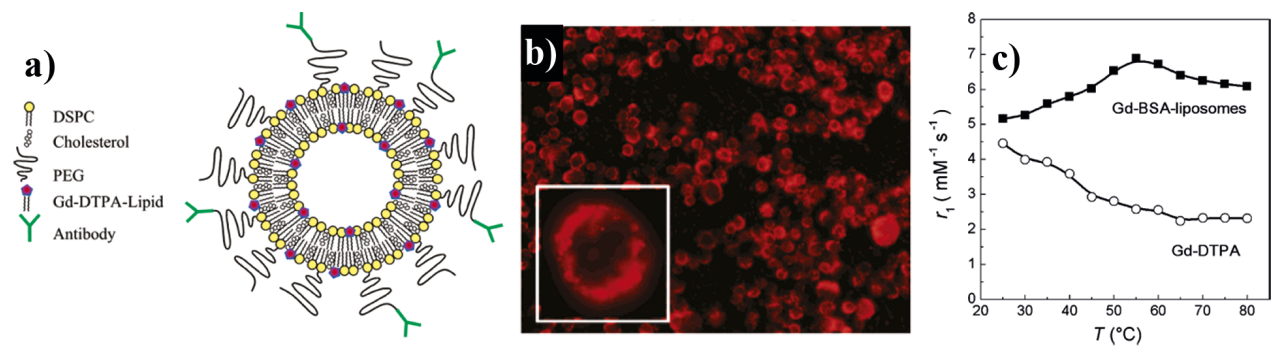

Figure 2.11. A) Schematic representation of a PEGylated paramagnetic liposome consisting of $\mathrm{Gd}^{3+}$-DTPA-BSA, DSPC, Chol and PEG-DSPE. Note that the antibodies are coupled to the distal end of the PEG-chains. B) TNF $\alpha$-treated HUVEC showing the immunoliposomes. Inset: fluorescence partly originates from the cell interior (magnification 630x). C) lonic $r_{1}$ relaxivities of free $\mathrm{Gd}^{3+}$-DTPA (O) and $\mathrm{Gd}^{3+}$-DTPA-BSA containing liposomes ( $\square$ ) as a function of the temperature at $300 \mathrm{MHz}$. Adapted with permission from. ${ }^{170}$ Copyright 2004 American Chemical Society.

Kamaly et al. ${ }^{171}$ used $\mathrm{Gd}^{3+}-2-\{4,7$-bis-carboxymethyl-10-[(N,Ndistearylamidomethyl-N'-amidomethyl]-1,4,7,10-tetraazacyclododec-1-yl\} acetic acid (DOTA-DSA) and rhodamine-labeled 1,2-dioleoyl-sn-glycero-3phosphoethanolamine (DOPE) in order to obtain $\mathrm{Gd}^{3+}$-liposomes suitable for cell labeling and tumor imaging (Figure 2.12a). After optimization to achieve maximum cellular entry, HeLa cells were successfully labeled in vitro. Whereas internalization by the cell was confirmed by optical imaging, (Figure 2.12b), uptake of the $\mathrm{Gd}^{3+}$-liposomes by the incubated cells resulted in lowered $T_{1}$ relaxation times (Figure 2.12c). 


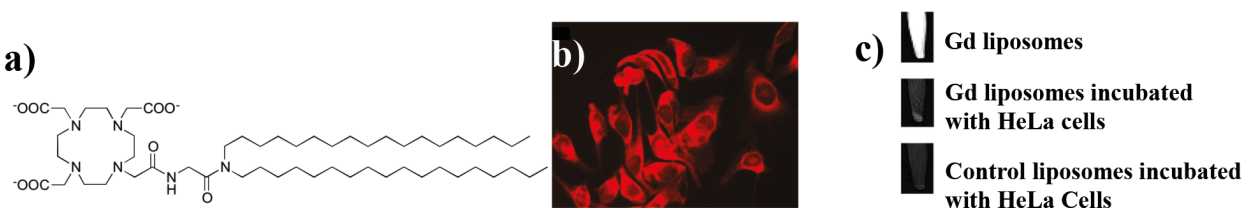

Figure 2.12. a) Chemical structure of the ligand DOTA-DSA. b) Fluorescence image of HeLa cells labeled with $\mathrm{Gd}^{3+}$-liposome formulation (40x magnification). c) $T_{1}$-weighted MR images of the $\mathrm{Gd}^{3+}$-liposome formulation solution (incubated HeLa cells and controls). Adapted with permission from. ${ }^{171}$ Copyright 2008 American Chemical Society.

Paramagnetic micelles of PEG-DSPE and $\mathrm{Gd}^{3+}$-DTPA-BSA with either rhodamine-PE or QDs have also been used for multimodality purposes. These micelles were conjugated with macrophage scavenger receptor (MSR)-specific antibodies by Mulder et al. ${ }^{172}$ The QD-containing micelles were used for identification of abdominal aortas of atherosclerotic apoE$\mathrm{KO}$ mice using UV light, while the rhodamine-PE containing micelles were used as trackers for cellular details by means of fluorescence microscopy (Figure 2.13a). $T_{1}$-weighted high-resolution MR images (before and $24 \mathrm{~h}$ after intravenous administration) showed successful imaging of the abdominal aortas (Figure 2.13b).

Metallostars. Moriggi et al., ${ }^{173}$ inspired by the paramagnetic metallostars published by Livramento et al., ${ }^{93}$ used the same " $\mathrm{Gd}^{3+}$-complex selfassembly around a transition metal center (iron)" concept in order to engineer a new class of dual IAs. Using two diethylenetriamine-N,N,N",N"'tetraacetate (DTTA) chelators integrated in a bipyridyl (bpy) ligand, six paramagnetic centers per molecule were obtained after complexation with ruthenium (Figure 2.14, left). The multimodal properties of the $\left\{R u\left[L n_{2} b p y-\right.\right.$ $\left.\operatorname{DTTA}_{2}\left(\mathrm{H}_{2} \mathrm{O}\right)_{4}\right]_{3} 3^{4-}$ metallostar were illustrated by the enhanced ionic $r_{1}$ relaxivity of the $\mathrm{Gd}^{3+}$ complex and the luminescence properties, due to 
sensitization of $\mathrm{Eu}^{3+}$ upon excitation of the tris(2,2'-bpy) $\mathrm{Ru}^{2+}$ unit, shown by the $\mathrm{Eu}^{3+}$ complex.

a)

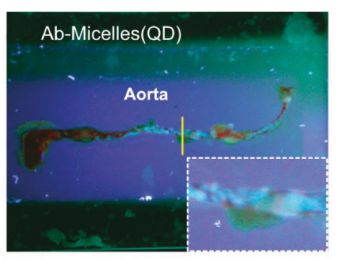

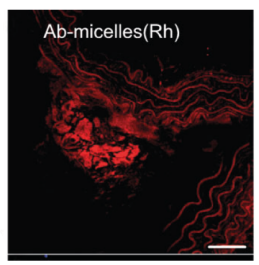

b)
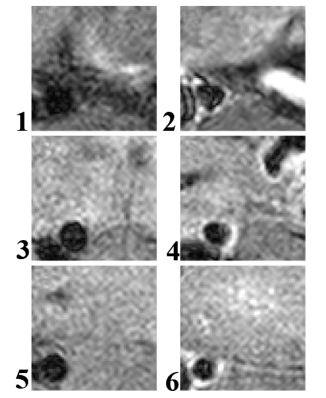

Figure 2.13. Left: a) Left: UV illumination image of an excised aorta (animal injected with QD-micelles) showing green fluorescence-emitting regions (inset). Right: Fluorescence microscopy image showing uptake of the rhodamine-labeled micelles. b) High-resolution $T_{1}$-weighted MR images before $(1,3$, and 5$)$ and $24 \mathrm{~h}$ after administration of rhodaminelabeled micelles (2) and QD-micelles (4 and 6). Adapted with permission from. ${ }^{172}$ Copyright 2007 John Wiley \& Sons.
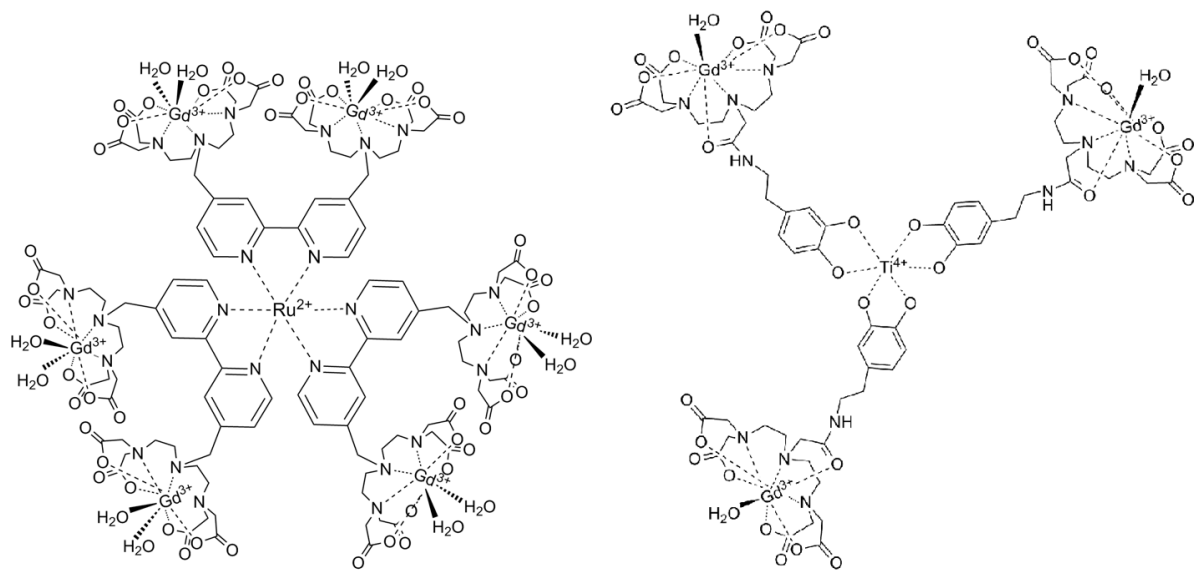

Figure 2.14. Left: structure of the self-assembling $\left\{R u\left[G d_{2} b p y-D T T A_{2}\left(H_{2} O\right)\right]_{3}\right\}^{4-}$ metallostar. ${ }^{173}$ Right: structure of the self-assembling $\left[\left(\mathrm{Gd}^{3+}-\mathrm{DTPA}\right)_{3} \mathrm{Ti}\left(\mathrm{H}_{2} \mathrm{O}\right)_{3}\right]^{5-}$ metallostar. ${ }^{174}$ 
More recently, Dehaen et al. ${ }^{174}$ engineered metallostars used a chelating ligand bearing a catechol and a DTPA moiety. In this way, lanthanide ions were complexed by the DTPA ligand while the metallostar forms as the with $\mathrm{Gd}^{3+}$ complexed ligands self-assemble around a titanium ion (Figure 2.14, right). The $\left[\left(\mathrm{Gd}^{3+}-\mathrm{DTPA}\right)_{3} \mathrm{Ti}\left(\mathrm{H}_{2} \mathrm{O}\right)_{3}\right]^{5-}$ metallostar showed multimodal properties demonstrated by enhanced ionic $r_{1}$ relaxivity upon selfassembly and broadband green emission (400-750 nm) upon UV irradiation.

\subsubsection{Inorganic Nanoparticles}

The attractiveness of inorganic nanoparticles (NPs) for multimodal imaging purposes lies not only in their intrinsic properties (optical detectability, therapeutical properties, etc.), but also in the large surface area-to-volume ratio they offer. This surface can be (relatively) easily modified by ligand exchange, making them excellent platforms for the integration of various IAs. Many inorganic NPs, such as quantum dots (QDs), gold and silica NPs have been successfully implemented for multimodal imaging purposes, as it will be revealed in the next sections. While the standard ionic $r_{1}$ relaxivity (value per $\mathrm{Gd}^{3+}$ ion) is still the mostly used way of expressing the relaxivity properties of such systems, the term "nanoparticle $r_{1}$ " is frequently referred to as a measure of the relaxivity per NP. There are no clear guidelines for the calculation of this value and it is usually done either by: i) calculating the relaxivity per NP concentration (using a standard inversion recovery experiment where instead of plotting $1 / T_{1}$ values as a function of the $\mathrm{Gd}^{3+}$ concentration, the NP concentration is used) or ii) by multiplying the calculated ionic $r_{1}$ relaxivity by the number of $\mathrm{Gd}^{+3}$ ions present. Throughout this review, the calculated nanoparticle $r_{1}$ value refers to the former procedure. 
Quantum Dots. Given the tunable optical properties of QDs, these inorganic NPs have been used as platforms to increase the local $\mathrm{Gd}^{3+}$ concentration and tumbling rate of paramagnetic complexes while, at the same time, making use of their optical detectability. ${ }^{8}$ Furthermore, QDs are excellent alternatives to fluorescent organic dyes, as these NPs can be used for imaging over long periods of time since they are resistant to photobleaching. ${ }^{175} \mathrm{~A}$ popular approach followed to develop multimodal IAs is the entrapment of QDs in paramagnetic supramolecular aggregates and some relevant examples will be discussed in the next sections. A review dealing with multimodal assemblies of amphiphiles and inorganic NPs for molecular imaging can be found elsewhere. ${ }^{176}$

Mulder et al. ${ }^{177}$ coated CdSe/ZnS core/shell, trioctylphosphineoxide/hexadecylamine (TOPO/HAD)-capped QDs with a micellular, paramagnetic coating consisting of PEG-DSPE and $\mathrm{Gd}^{3+}$-DTPABSA (Figure 2.15a). By conjugating the fluorescent QDs to cyclic, $\alpha_{v} \beta_{3}$ specific RGD peptides, their specificity for angiogenic blood vessels was tested (Figure 2.15b). In vitro studies on cultured HUVEC showed an ionic relaxivity $r_{1}$ for these QDs of $12 \mathrm{mM}^{-1} \mathrm{~s}^{-1}\left(3 \times \mathrm{Gd}^{3+}\right.$-DTPA) and a particle relaxivity of $2000 \mathrm{mM}^{-1} \mathrm{~s}^{-1}$, corresponding to $\sim 150 \mathrm{Gd}^{3+}$-DTPA-BSA lipids (Figure 2.15c). 
a)

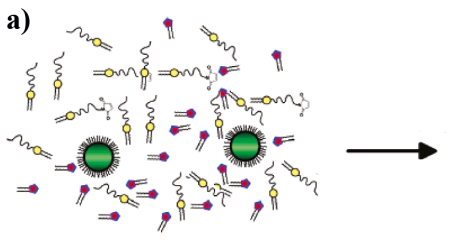

b)

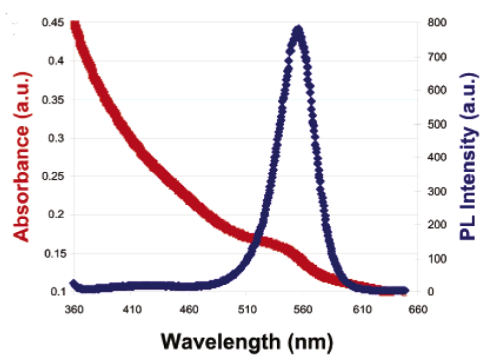

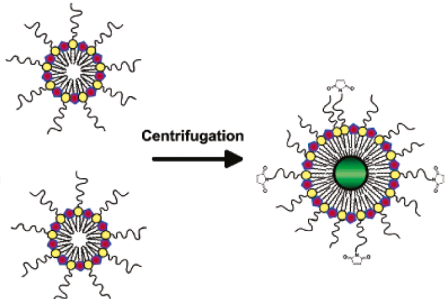

c)

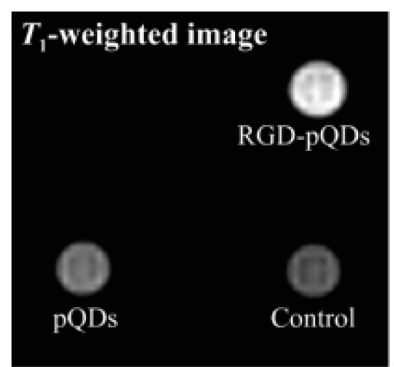

Figure 2.15. a) Schematic representation of the preparation of paramagnetic, micellecoated QDs. b) UV-Vis absorption (red) and PL (blue) spectra of micelle-coated QDs. c) $T_{1^{-}}$ weighted image (at $60 \mathrm{MHz}$ ) of cells incubated with RGD-QDs, QDS, or QDs (no CA). Adapted with permission from. ${ }^{177}$ Copyright 2006 American Chemical Society.

In another example, Van Tilborg et.al. ${ }^{178}$ used CdSe/ZnS core/shell, TOPO/HDA-capped QDs coated with a mixture of paramagnetic $\mathrm{Gd}^{3+}$ DTPA-bis(stearylamide) (Gd ${ }^{3+}$-DTPA-BSA) and 1,2-distearoyl-sn-glycero-3phosphoethanolamine-N-[methoxy(poly(ethylene glycol))-2000] (PEGDSPE) micelles. Multiple recombinant human annexin A5 protein molecules (AnxA5) were covalently attached to maleimide-labeled PEGDSPE (Figure 2.16a). These multimodal AnxA5-conjugated NPs (AnxA5pQD) were fluorescent (Figure 2.16b) and showed enhanced paramagnetic properties, with an ionic $r_{1}$ of $12.4 \mathrm{mM}^{-1} \mathrm{~s}^{-1}$ and a particle relaxivity at least 150 times higher (Figure 2.16c). The potential use of AnxA5-pQDs as in vivo probes for apoptotic cell detection was tested and fluorescence microscopy and MRI demonstrated the successful attachment and targeting. 
a)

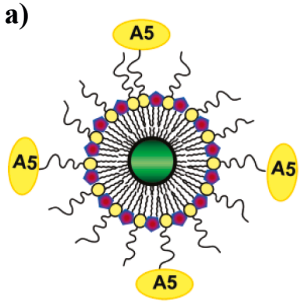

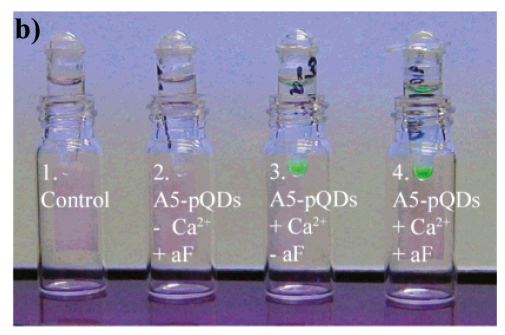

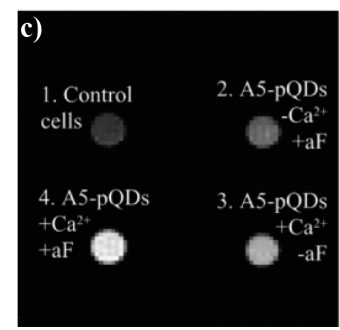

Figure 2.16. a) Schematic representation of AnxA5-pQD. The lipids drawn with the yellow and red head groups represent PEG-DSPE and $\mathrm{Gd}^{3+}$-DTPA-BSA, respectively. b) Cell pellets of the different incubations illuminated with $254 \mathrm{~nm}$ UV light. From left to right: Control cells, apoptotic cells incubated with AnxA5-pQDs, vital cells incubated with AnxA5pQDs, and apoptotic cells incubated with AnxA5-pQDs. c) $T_{1}$-weighted image of the different cell pellets. Adapted with permission from. ${ }^{178}$ Copyright 2006 American Chemical Society.

Another way of engineering multimodal IAs using QDs as fluorescent probes is by surface modification. Prinzen et al. ${ }^{179}$ combined QDs with paramagnetic macromolecules and AnxA5 in order to synthesize multimodal NPs with increased $\mathrm{Gd}^{3+}$ payloads. Using the biotinstreptavidin linkage, streptavidin coated QDs (with an estimated 10 streptavidin molecules per QD) were used to obtain two kinds of NPs: a) single-biotinylated AnxA5 QDs prepared using a 1:1 stoichiometry (AnxA5QD-Gd), with all remaining binding sites filled with biotinylated $\mathrm{Gd}^{3+}$-DTPA and b) $\mathrm{Gd}^{3+}$-lysine wedge biotinylated QDs (AnxA5-QD-Gd-wedge, Figure 2.17), where each wedge contains $8 \mathrm{Gd}^{3+}$ chelates. These QD-based NPs exhibited intense fluorescence and the high $\mathrm{Gd}^{3+}$ payloads were evidenced by the nanoparticle $r_{1}$ relaxivities measured, which were $420-630 \mathrm{mM}^{-1} \mathrm{~s}^{-1}$ for AnxA5-QD-Gd and 3000-4500 $\mathrm{mM}^{-1} \mathrm{~s}^{-1}$ for AnxA5-QD-Gd-wedge. 


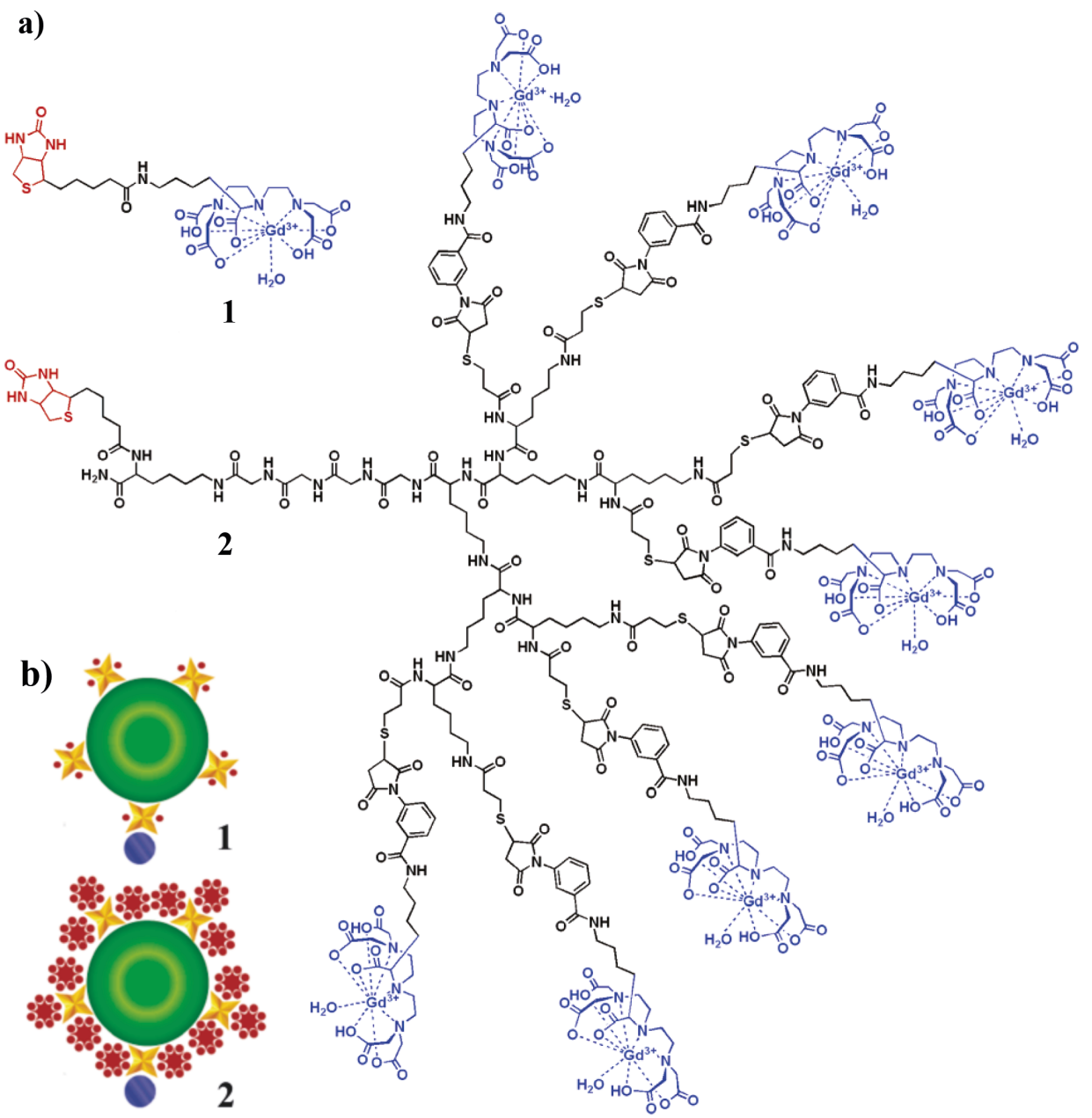

Figure 2.17. a) Chemical structure of biotinylated $\mathrm{Gd}^{3+}$-DTPA (1) and biotinylated $\mathrm{Gd}^{3+}-$ lysine wedge (2). Biotin (red) and $\mathrm{Gd}^{3+}$-DTPA (blue). b) Schematic representation of the two NPs: single biotinylated $\mathrm{Gd}^{3+}$-DTPA QD (1, AnxA5-QD-Gd) and $\mathrm{Gd}^{3+}$-lysine-wedge biotinylated QD (2, AnxA5-QD-Gd-wedge). Green: QD; yellow: streptavidin; red dot: Gd $^{3+}$ DTPA; red star: lysine-wedge; blue: AnxA5. Adapted with permission from. ${ }^{179}$ Copyright 2006 American Chemical Society.

Yang et al. ${ }^{103}$ functionalized water-soluble silica-coated ZnS-passivated CdS:Mn fluorescent QDs with tetraethyl orthosilicate (TEOS), 3- 
(aminopropyl) triethoxysilane (APTS) and 3-(trihydroxysilyl) propyl methylphosphonate (THPMP). Chelates for $\mathrm{Gd}^{3+}$ complexation were added by further functionalization with $n$-(trimethoxysilylpropyl)ethyldiamine, triacetic acid trisodium salt (TSPETE), a metal chelating carboxylate silane coupling agent known to have five reactive coordination sites for $\mathrm{Gd}^{3+}$ (Figure 2.18). Local $\mathrm{Gd}^{3+}$ concentration enhancement was evidenced by the $107 \mathrm{Gd}^{3+}$ ions per QD measured. The high ionic $r_{1}$ relaxivity measured $\left(20.5 \mathrm{mM}^{-1} \mathrm{~s}^{-1}\right)$ was attributed to the lowered tumbling rate due to the strong anchoring of the $\mathrm{Gd}^{3+}$-coordinated TSPETE to the rigid silica coated QDs. The $\mathrm{Gd}^{3+}$-labeled QDs showed fluorescence emission at $590 \mathrm{~nm}\left(\lambda_{\mathrm{ex}}\right.$ $345 \mathrm{~nm})$.

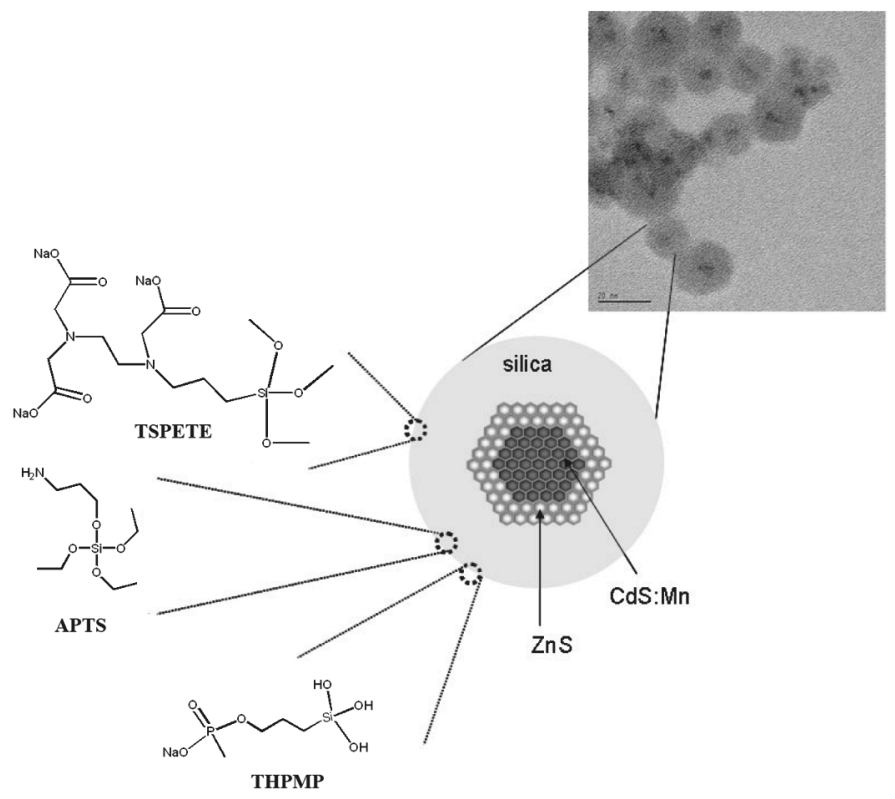

Figure 2.18. Schematic representation of a silica-coated CdS:Mn/ZnS core/shell QD functionalized using APTS, THPMP, and TSPETE. Upper right corner: TEM image of $\mathrm{Gd}^{3+}-$ QD. Scale bar is $20 \mathrm{~nm}$. Adapted with permission from. ${ }^{103}$ Copyright 2006 John Wiley \& Sons. 
A synthetically less demanding approach has also been proposed by Jin et al. ${ }^{101}$ To glutathione (GSH, reduced form) coated CdSeTe/CdS QDs, $\mathrm{Gd}^{3+}$ DOTA (1,4,7,10-tetraazacyclododecane-1,4,7,10-tetraacetic acid) was covalently attached by means of an amide bond. These multimodal QDs, which retain $60 \%$ of their fluorescence after being coated with $\mathrm{GSH}$, emitted in the NIR region and showed enhanced positive MRI contrast when compared to GSH-QDs alone (Figure 2.19).

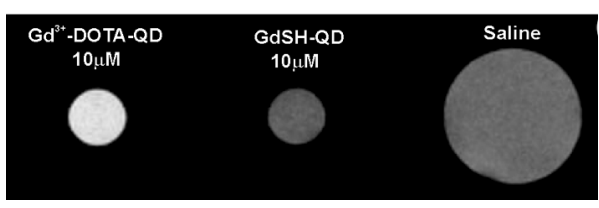

Figure 2.19. $T_{1}$-weighted $M R$ images for saline, GSH-QDs and $\mathrm{Gd}^{3+}$-DOTA-QDs in PBS. Adapted from. ${ }^{101}$

Other chelators used for $\mathrm{Gd}^{3+}$ complexation include S-2-(4isothiocyanatobenzyl)-1,4,7,10-tetraazacyclododecane tetraacetic acid (DOTA-Bn-NCS), used by Bakalova et al. ${ }^{180}$ and DDTA, used by Park et al (Figure 2.20a and $b$ respectively). ${ }^{181}$ The latter DTTA-ligated uridine-QD conjugates showed enhanced nanoparticle $r_{1}$ relaxivities (655 and 571.2 $\mathrm{mM}^{-1} \mathrm{~s}^{-1}$ at 60 and $200 \mathrm{MHz}$, respectively), payloads of approximately $56 \pm$ $6 \mathrm{Gd}^{3+}$ ions per particle and confirmed uptake by RAW 264.7 cells. 
a)

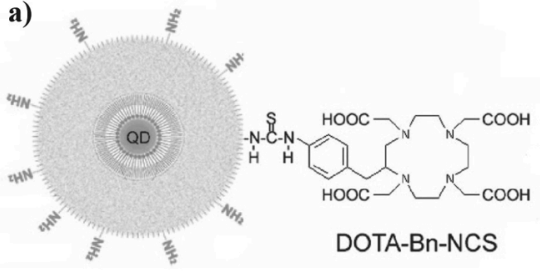

b)

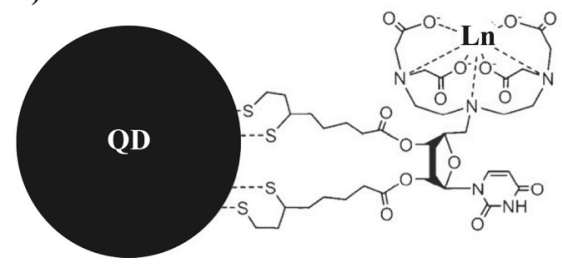

Figure 2.20. a) Schematic representation of multimodal silica-shelled QD probes: the $\mathrm{Gd}^{3+}$ complex is conjugated on the surface of the silica sphere. Reprinted with permission from. ${ }^{180}$ Copyright 2008 American Chemical Society. b) Schematic representation of DTTAligated uridine coated QDs. Adapted from. ${ }^{181}$

Gold Nanoparticles. Gold NPs (AuNPs) are very attractive for biological applications due to their light absorption and scattering properties derived from localized surface plasmon resonance (LSPR), observed as strong extinction peaks in the visible and near-infrared regions. Song et al. ${ }^{182}$ used AuNPs in order to create multimodal, $\mathrm{Gd}^{3+}$ enriched polyvalent DNA-AuNP conjugates (DNA-Gd $\left.{ }^{3+} @ A u N P\right)$. These AuNPs were prepared by reacting citrate-stabilized AuNPs with thiol-labeled 24-mer poly-dT oligonucleotides, the latter containing five conjugation sites for covalent attachment of $\mathrm{Gd}^{3+}$-complexes. The multimodal Cy3-DNA-Gd ${ }^{3+} @ A u N P s$ were obtained by conjugation of Cy3 to the $5^{\prime}$ end of the DNA-Gd ${ }^{3+}$ strands oligomers (Figure 2.21a). While cellular uptake and efficient labeling was confirmed by fluorescence microscopy (Figure 2.21b), the calculated ionic $r_{1}$ relaxivity of the $\mathrm{Gd}^{3+}$ complex after conjugation to DNA $\left(8.7 \mathrm{mM}^{-1} \mathrm{~s}^{-1}\right.$ at $60 \mathrm{MHz}$ ) was twice as high as that of the unconjugated $\mathrm{Gd}^{3+}$ complex (Figure 2.21c). 
a)

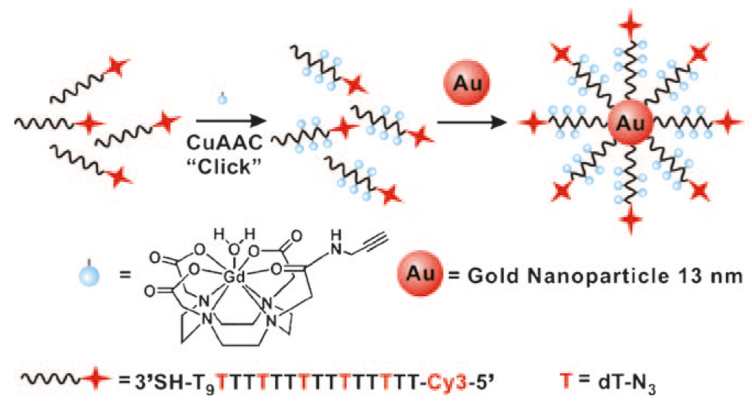

b)

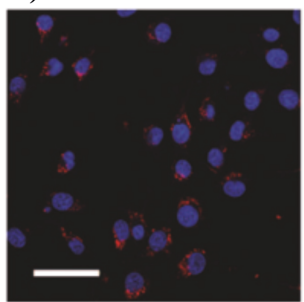

c)

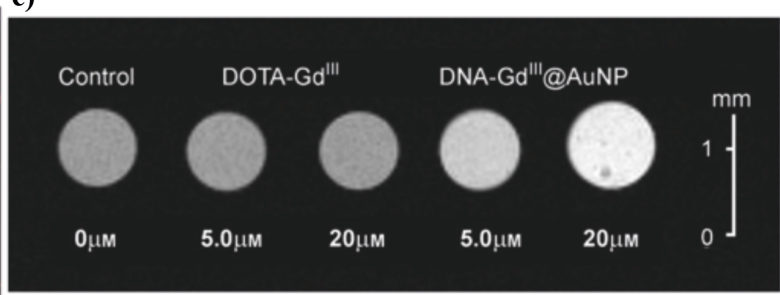

Figure 2.21. a) Synthesis of Cy3-DNA-Gd ${ }^{3+} @ A u N P$ conjugates. b) Confocal fluorescence micrograph of NIH/3T3 cells incubated with $0.2 \mathrm{nM}$ particle concentration of Cy3-DNA$\mathrm{Gd}^{3+} @$ AuNPs (red channel) for $4 \mathrm{~h}$ and a $24 \mathrm{~h}$ leach in fresh media and $1 \mu \mathrm{m}$ DAPI $\left(4^{\prime}, 6-\right.$ diamidino- 2-phenylindole, blue channel) for $10 \mathrm{~min}$. c) $T_{1}$-weighted MR image of NIH/3T3 cells incubated with 5 and $20 \mu \mathrm{M}$ (Gd ${ }^{3+}$ concentration) of DNA-Gd ${ }^{3+} @ A u N P$ and $\mathrm{Gd}^{3+}-$ DOTA for $24 \mathrm{~h}$ at $600 \mathrm{MHz}$. Adapted with permission from. ${ }^{182}$ Copyright 2009 John Wiley \& Sons.

Other Inorganic Nanoparticles. Bimodal silica NPs $\left(\mathrm{SiO}_{2} \mathrm{NPs}\right)$, recently proposed by Pinho et al., ${ }^{183}$ consist of $\mathrm{SiO}_{2}$ NPs coated with a linker, 3aminopropyltriethoxysilane (APS), used to covalently attach DTPA ligands for lanthanide complexation (Figure 2.22a). Studies using hybrid $\mathrm{SiO}_{2} @ \mathrm{APS} / \mathrm{DTPA}: \mathrm{Eu}^{3+} \mathrm{Gd}^{3+}(1: 1)$ and $\mathrm{SiO}_{2} @ \mathrm{APS} / \mathrm{DTPA}: \mathrm{Tb}^{3+} \mathrm{Gd}^{3+}$ (1:1) NPS showed that the optical properties of $\mathrm{Eu}^{3+}$ and $\mathrm{Tb}^{3+}$ were not influenced by the presence of $\mathrm{Gd}^{3+}$. Also, the moderate ionic $r_{1}$ increase observed confirmed the bimodal character of these NPs. Uptake studies on living cells showed increase intensity in the $T_{1}$-weighted $\mathrm{MRI}$ images as well as optical detection (Figure 2.22b and c respectively). The same principle has 
been used in order to make hybrid NPs with antenna-incorporated ligands, e.g., 2,2',2",2"'-[(pyridine-2,6-diyl)bis(methylenenitrilo)]tetra-kis(acetic acid) (PMN), showing comparable relaxometric and emission properties. ${ }^{184}$

a)

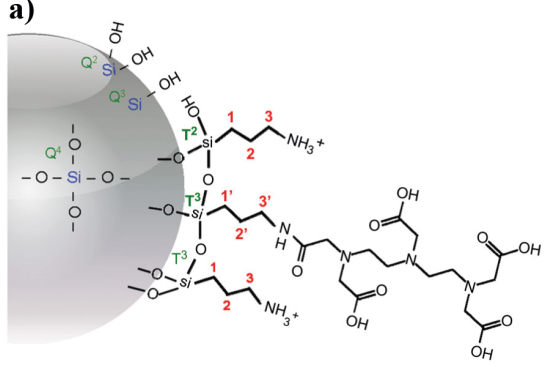

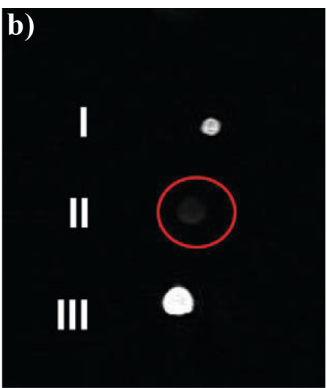

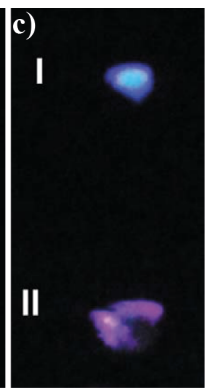

Figure 2.22. a) Schematic representation of a $\mathrm{SiO}_{2}$ NP functionalized with APS and coupled with DPTA. b) $T_{1}$-weighted MR image of cellular pellets corresponding to: I) no NPs internalization (control); II) $\quad \gamma-\mathrm{Fe}_{2} \mathrm{O}_{3} \quad \mathrm{NPS} \quad\left(T_{2}\right) \quad \mathrm{NPS}$ cell internalization and III) $\mathrm{SiO}_{2} @ A P S / D T P A: \mathrm{Eu}^{3+} \mathrm{Gd}^{3+}$ NPs cell internalization. c) Photograph of cellular pellets, excited at $393 \mathrm{~nm}$, corresponding to: I) no NPs cell internalization (control) and II) $\mathrm{SiO}_{2} @ \mathrm{APS} / \mathrm{DTPA}: \mathrm{Eu}^{3+} \mathrm{Gd}^{3+} \mathrm{NPs}$ cell incorporation. Adapted with permission from. ${ }^{183}$ Copyright 2012 Elsevier.

Another type of hybrid silica NPs has been studied by Rieter et al. ${ }^{185}$ These NPs contain a luminescent $\left[\mathrm{Ru}(\mathrm{bpy})_{3}\right] \mathrm{Cl}_{2}$ core (emission at $595 \mathrm{~nm}$ ) and a paramagnetic monolayer coating consisting of single or double silylated $\mathrm{Gd}^{3+}$-DTTA complexes (1 and 2 respectively, Figure 2.23, left). While NP 1 contained around $10200 \mathrm{Gd}^{3+}$ ions and showed an enhanced ionic $r_{1}$ relaxivity of $19.7 \mathrm{mM}^{-1} \mathrm{~s}^{-1}$, an increase in the number of $\mathrm{Gd}^{3+}$ ions did not lead to higher relaxivities, as NP 2 contained $63200 \mathrm{Gd}^{3+}$ ions and showed an ionic $r_{1}=7.8 \mathrm{mM}^{-1} \mathrm{~s}^{-1}$. This decrease in ionic $r_{1}$ is attributed to the nonaccessible $\mathrm{Gd}^{3+}$ centers in the inner layers of $\mathbf{2}$. Also, studies using monocyte cells showed efficient uptake of these NPs, which was confirmed by optical and MR imaging (Figure 2.23, right). 

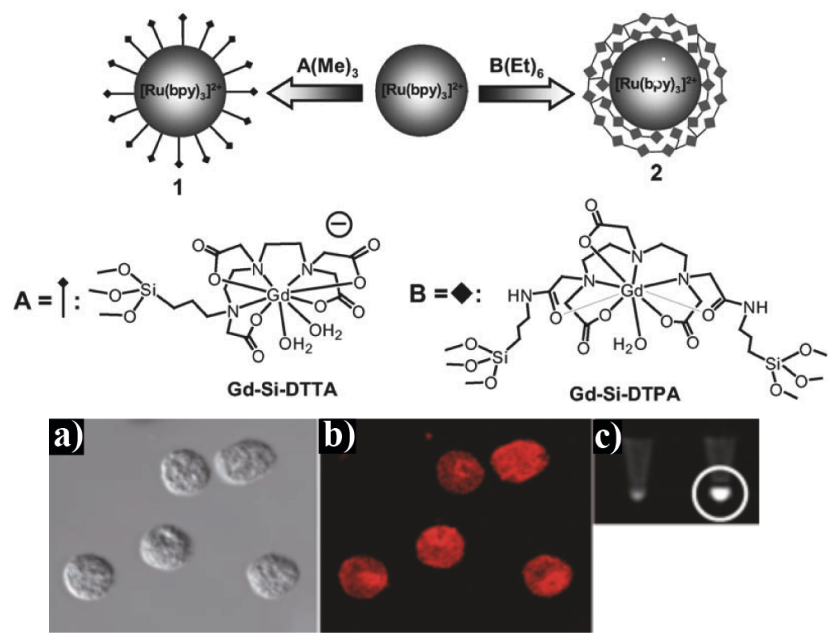

Figure 2.23. Above: schematic representations of hybrid $\left[\mathrm{Ru}(\mathrm{bpy})_{3}\right] \mathrm{Cl}_{2}$ core + silylated $\mathrm{Gd}^{3+} \mathrm{DTTA}$ complex NPs. Below: images of 1-labeled monocyte cells: a) optical microscopy; b) laser scanning confocal fluorescence microcopy; c) $T_{1}$-weighted MR image of unlabeled (left) and 1-labeled (right) monocyte cells. Adapted with permission from. ${ }^{185}$ Copyright 2007 John Wiley \& Sons.

\subsubsection{Nanocontainers}

Surface modification of NPs by means of ligand exchange is not the only method available for multimodal imaging purposes, as nanostructures can also be used as scaffolds where IAs are "entrapped". Examples of both organic and inorganic nanocontainers include organic NPs/protein cages and zeolites, respectively.

Organic Nanoparticles. Nam et al. ${ }^{186}$ recently introduced tumor targeting, self-assembled chitosan NPs (CNPs) for optical and MR imaging. Amphiphilic glycol chitosan $5 \beta$-cholanic acid conjugates were reacted with Cy5.5-NHS (Figure 2.24a) and monoreactive DOTA-NHS in order to obtain $\mathrm{Gd}^{3+}$-encapsulated conjugates. Spontaneous self-assembly of these conjugates in water led to stable, spherical encapsulated chitosan 
NPs (Cy5.5-CNP-Gd ${ }^{3+}$ ) of approx. $350 \mathrm{~nm}$ in diameter (Figure 2.24b) with strong NIR fluorescence emission (Figure 2.24c, left). Contrast enhancement in $T_{1}$-weighted $\mathrm{MR}$ images showed the paramagnetic properties of these CNPs. Whereas CNPs showed similar enhancement at different $\mathrm{pH}$ conditions (Figure 2.24c, right), encapsulation of the $\mathrm{Gd}^{3+}$ chelates in the hydrophobic inner part of the CNPs did affect the ionic $r_{1}$ relaxivity. Likewise, chitosan has also been used to encapsulate both CdSe/ZnS QDs and $\mathrm{Gd}^{3+}$-DTPA, showing optical properties and relatively high relaxivity. ${ }^{187}$

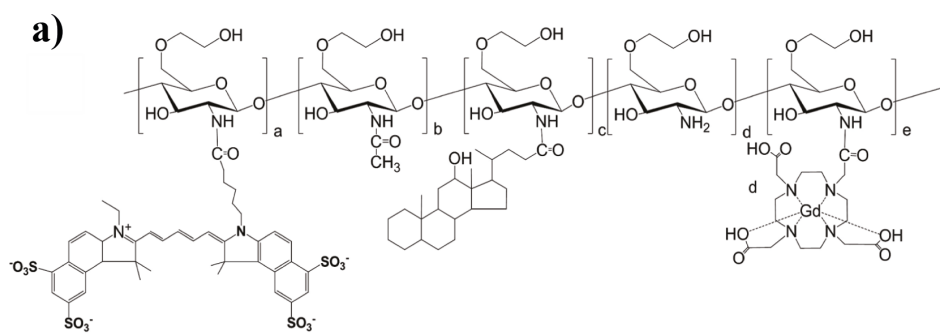

b)

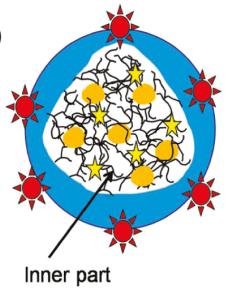

L Glycol chitosan If polymer

Hydrophobic $5 \beta-$ cholanic acids NIRF Cy5.5 Gd(III) c)

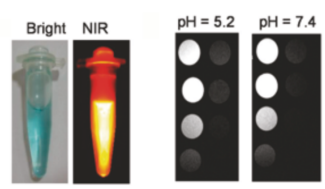

Cy5.5-CNP-Gd(III)

Figure 2.24. a) Chemical structure of Cy5.5-CNP-Gd ${ }^{3+}$. b) Schematic representation of the self-assembled chitosan NPs CNPs. Blue: chitosan shell; white: inner part CNP. c) Left: NIR-fluorescence images of the Cy5.5-CNP-Gd ${ }^{3+}$ in PBS. Right: $T_{1}$-weighted spin-echo images of Cy5.5-CNP-Gd ${ }^{3+}$ in acidic $(\mathrm{pH} 5.2)$ and physiological conditions $(\mathrm{pH}$ 7.4). Adapted with permission from. ${ }^{186}$ Copyright 2010 American Chemical Society.

Protein Cages. Owing to their extensive surface and reactive groups available for covalent functionalization, protein cages or virus-like particles (VLP) have been explored as possible carriers of IAs. Anderson et al. ${ }^{188}$ 
conjugated $\mathrm{Gd}^{3+}$ complexes onto MS2 viral capsids using lysine-reactive 2-(4-isothiocyanatobenzyl)-diethylenetriaminepentaacetic acid (DTPA-ITC). At the same time, VLPs were reacted with fluorescein isothiocyanate (FITC) in order to obtain the multimodal MS2 $\left(\mathrm{Gd}^{3+}-\mathrm{DTPA}-\mathrm{ITC}\right)_{\mathrm{m}}(\mathrm{FITC})_{\mathrm{n}}$ viral capsids (Figure 2.25a). The paramagnetic properties of these labeled VLPS were clearly enhanced (Figure 2.25b), with a calculated ionic $r_{1}$ relaxivity for the bimodal MS2 $\left(\mathrm{Gd}^{3+} \text {-DTPA-ITC }\right)_{360}(\mathrm{FITC})_{55}$ of $16.9 \mathrm{mM}^{-1} \mathrm{~s}^{-1}$ and a nanoparticle $r_{1}$ of $6100 \mathrm{mM}^{-1} \mathrm{~s}^{-1}$ (at $60 \mathrm{MHz}$ ). Using the same approach, Suci et al. ${ }^{189}$ attached $N$-hydroxysuccinimide derivatives of fluorescein and DOTA to multifunctionalize cowpea chlorotic mottle virus (CCMV) protein cages. This multimodal platform was used to observe penetration into a Staphylococcus aureus film, thereby demonstrating their possible application as diagnostic and targeted treatment agents for bacterial infections.

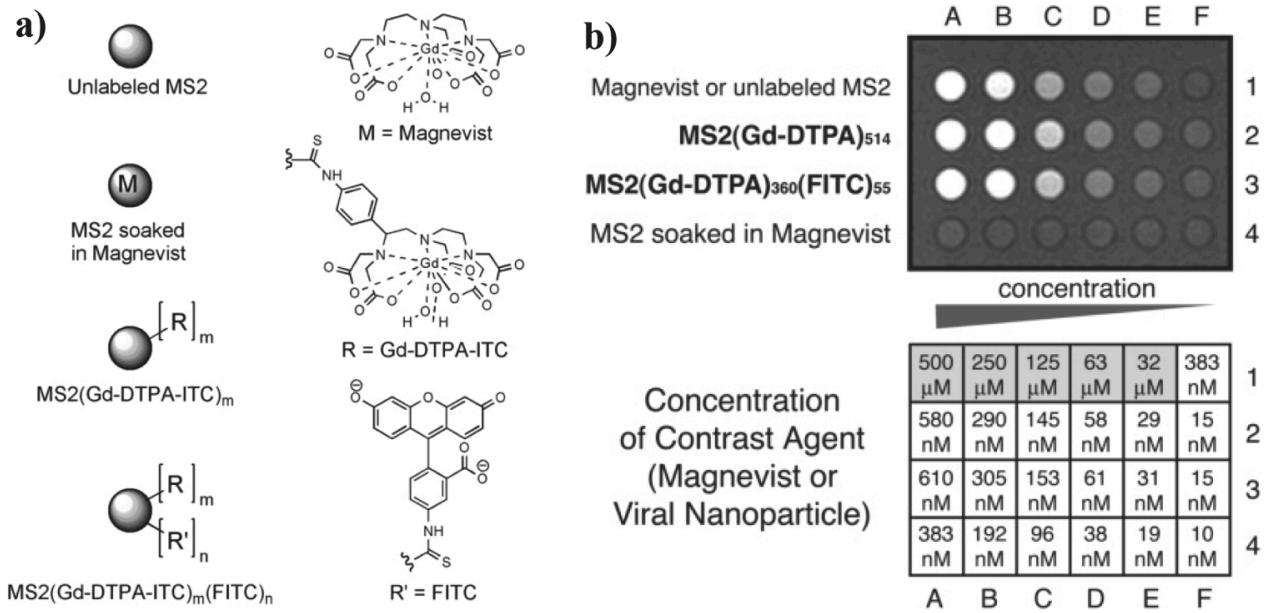

Figure 2.25. a) VLPs used for MRI studies: MS2 capsids, MS2 capsids soaked in Magnevist (control), paramagnetic MS2 $\left(\mathrm{Gd}^{3+} \text {-DTPA-ITC }\right)_{\mathrm{m}}$ and bimodal MS2 $\left(\mathrm{Gd}^{3+}\right.$-DTPAITC $)_{m}(F I T C)_{n}(m+n \approx 540)$. b) $T_{1}$-weighted image of the different VLPs. Adapted with permission from. ${ }^{188}$ Copyright 2006 American Chemical Society. 
Pokorski et al. ${ }^{190}$ increased the number of functionalities that can be attached to a VLP by using these protein cages as multivalent macroinitiators for atom transfer radical polymerization (ATRP). VPLs functionalized with oligo(ethylene glycol)-methacrylate (OEGMA) and its azido-functionalized analogue (OEGMA- $\mathrm{N}_{3}$ ) can be easily modified by means of $\mathrm{Cu}(\mathrm{l})$-catalyzed click chemistry (CuAAC). By allowing the poly $\left(\mathrm{OEGMA}-\mathrm{N}_{3}\right)$ grafts (1) to react with alkyne-substituted Alexa Fluor 488 dyes and DOTAs (under CuAAC conditions), multimodal VPLs were obtained (Figure 2.26a). Successful incorporation of paramagnetic moieties to these VLPs was confirmed by the $r_{1}$ relaxivity per particle calculated for $5\left(7092 \mathrm{mM}^{-1} \mathrm{~s}^{-1}\right)$, leading to values comparable to other covalently attached $\mathrm{Gd}^{3+}$ complex VLPs.

a)

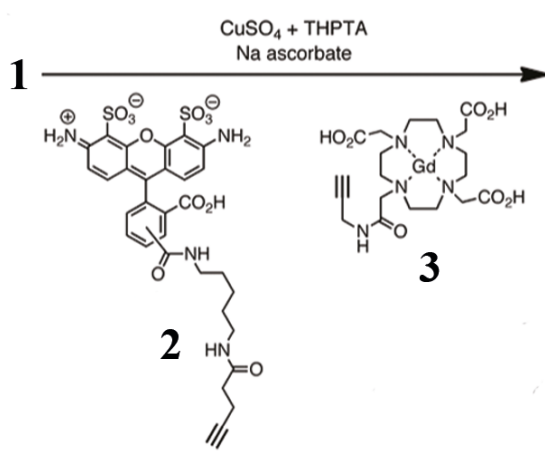

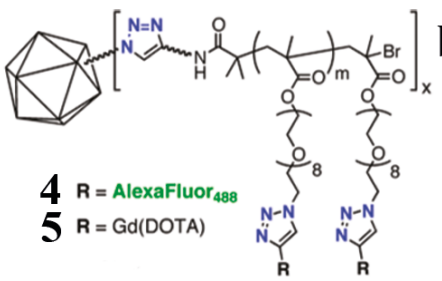

b)

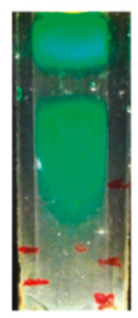

Figure 2.26. a) CuAAC reaction to attach the different functionalities to 1. b) UV illumination of sucrose gradient following CuAAC between 1 and $\mathbf{2}$ (upper band, unreacted 2; lower band, 4). Adapted with permission from. ${ }^{190}$ Copyright 2011 American Chemical Society.

Zeolites. Microporous inorganic scaffolds such as zeolites, characterized by their biocompatibility and high active surface available, have also been explored as platforms for the integration of various imaging modalities. Tsotsalas et al. ${ }^{191}$ used zeolite $L$ crystals as dual optical and paramagnetic 
probes. The channels of the zeolite crystals were filled with pyronine molecules by means of exchange of potassium cations. An APTS linker was then reacted with the free $\mathrm{Si}-\mathrm{OH}$ groups on the zeolite surface and subsequently coupled to DOTA-NHS. The paramagnetic zeolites were obtained by complexation of the DOTA ligand with $\mathrm{Gd}^{3+}$ (Figure 2.27). The enhanced paramagnetic properties of these nanocontainers are evidenced by the high ionic $r_{1}$ relaxivity measured $\left(30 \mathrm{mM}^{-1} \mathrm{~s}^{-1},>6 x\right.$ larger than $\mathrm{Gd}^{3+}-$ DOTA) and the calculated zeolite overall relaxivity (ca. $11000 \mathrm{mM}^{-1} \mathrm{~s}^{-1}$ ).

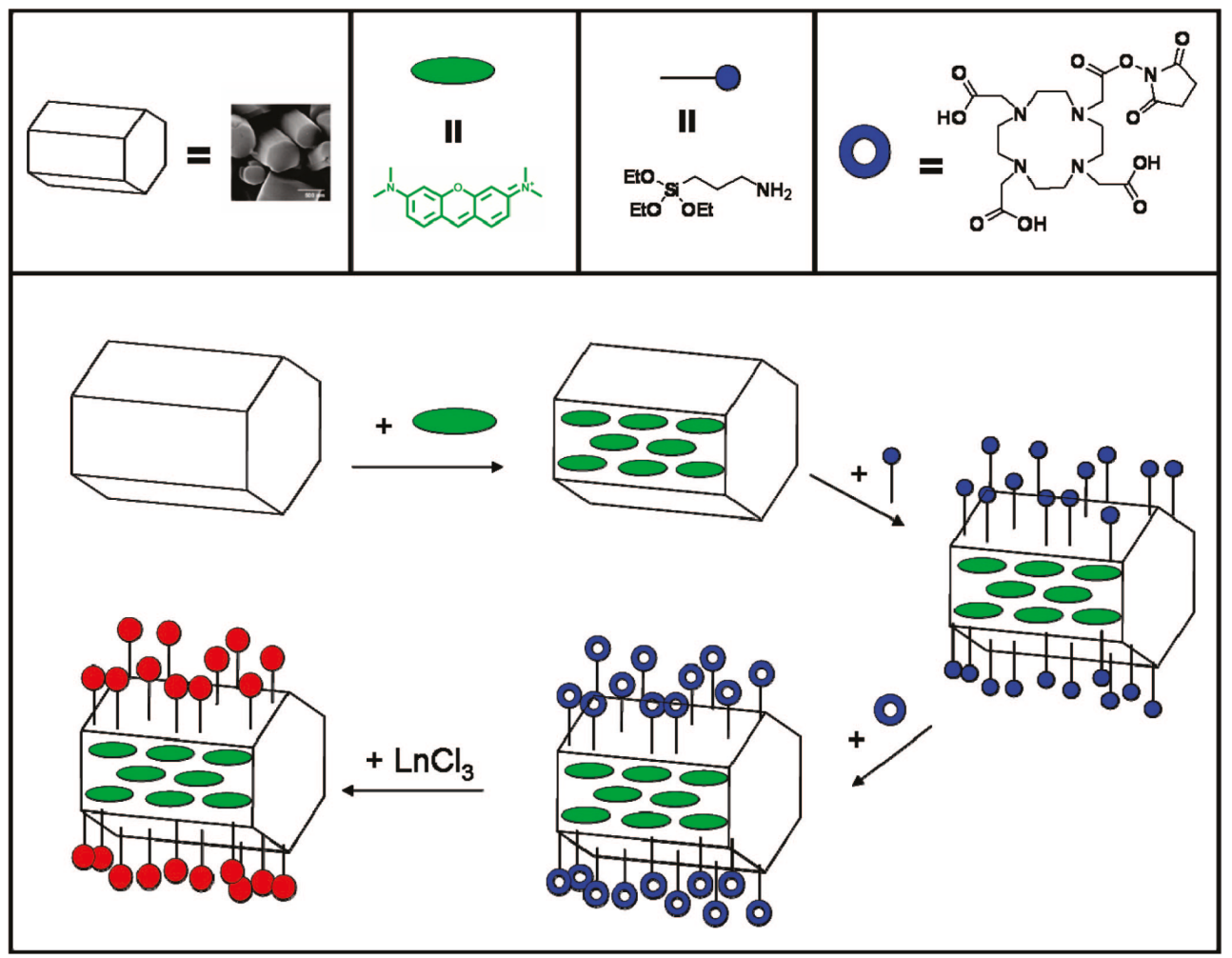

Figure 2.27. Synthesis of $\mathrm{Gd}^{3+}$-DOTA functionalized zeolite $\mathrm{L}$ crystals. First, pyronine molecules are introduced into the channels via ion exchange (green ovals). Then, APTS (linker) is bound to the entire surface of the zeolite L crystals (blue dot). Subsequently, the DOTA ligand is covalently attached to the backside of the linker (blue ring). Finally, the lanthanide $\left(\mathrm{Gd}^{3+}\right)$ ions are complexed to the surface of the zeolite $\mathrm{L}$ crystals (red balls). Adapted with permission from. ${ }^{191}$ Copyright 2008 American Chemical Society. 


\subsection{Conclusions}

Despite the increasing number of imaging modalities available nowadays, the use of more traditional imaging techniques such as MRI, PET, SPECT and optical imaging is still favored. Preference for the combination MR/optical imaging is illustrated by the large number of publications dealing with multimodal IAs for these two techniques shown in this literature survey. Innovative development of multimodal probes is triggered by the rapidly growing knowledge of chemistry of IAs, nanomaterials and molecular self-assembly. In this thesis, new approaches for the development of IAs, inspired by novel nanomaterials and molecular selfassembly, for MR and/or optical imaging are described.

\subsection{References}

(1) Galbán, C. J.; Galbán, S.; Van Dort, M. E.; Luker, G. D.; Bhojani, M. S.; Rehemtulla, A.; Ross, B. D. In Progress in Molecular Biology and Translational Science; Raymond, W. R., Ed.; Academic Press: 2010; Vol. Volume 95, p 237.

(2) Hausner, S. H. In Nanoplatform-Based Molecular Imaging; Chen, X., Ed.; John Wiley \& Sons, Inc.: 2011, p 1.

(3) Weissleder, R.; Mahmood, U. Radiology 2001, 219, 316.

(4) Cassidy, P. J.; Radda, G. K. J. Royal Soc. Interface 2005, 2, 133.

(5) Brindle, K. Nat. Rev. Cancer 2008, 8, 94.

(6) Seaman, M. E.; Contino, G.; Bardeesy, N.; Kelly, K. A. Expert Rev. Mol. Med. 2010, 12, 1.

(7) Brücher, E. In Top Curr Chem; Krause, W., Ed.; Springer Berlin / Heidelberg: 2002; Vol. 221, p 103.

(8) Louie, A. Chem. Rev. 2010, 110, 3146.

(9) Frullano, L.; Meade, T. J. Biol. Inorg. Chem. 2007, 12, 939.

(10) Jennings, L. E.; Long, N. J. Chem. Commun. 2009, 3511.

(11) Geninatti Crich, S. G.; Biancone, L.; Cantaluppi, V.; Esposito, D. D. G.; Russo, S.; Camussi, G.; Aime, S. Magn. Reson. Med. 2004, 51, 938.

(12) Picard, C.; Geum, N.; Nasso, I.; Mestre, B.; Tisnes, P.; Laurent, S.; Muller, R. N.; Vander Elst, L. Bioorg. Med. Chem. Lett. 2006, 16, 5309.

(13) Judenhofer, M. S.; Wehrl, H. F.; Newport, D. F.; Catana, C.; Siegel, S. B.; Becker, M.; Thielscher, A.; Kneilling, M.; Lichy, M. P.; Eichner, M.; Klingel, K.; 
Reischl, G.; Widmaier, S.; Rocken, M.; Nutt, R. E.; Machulla, H.-J.; Uludag, K.; Cherry, S. R.; Claussen, C. D.; Pichler, B. J. Nat. Med. 2008, 14, 459.

(14) Catana, C.; Wu, Y.; Judenhofer, M. S.; Qi, J.; Pichler, B. J.; Cherry, S. R. J. Nucl. Med. 2006, 47, 1968.

(15) Cherry, S. R.; Louie, A. Y.; Jacobs, R. E. P. IEEE 2008, 96, 416.

(16) Choi, J. S.; Park, J. C.; Nah, H.; Woo, S.; Oh, J.; Kim, K. M.; Cheon, G. J.; Chang, Y.; Yoo, J.; Cheon, J. Angew. Chem., Int. Ed. 2008, 47, 6259.

(17) Culver, J.; Akers, W.; Achilefu, S. J. Nucl. Med. 2008, 49, 169.

(18) Cai, W.; Chen, K.; Li, Z.-B.; Gambhir, S. S.; Chen, X. J. Nucl. Med. 2007, 48, 1862.

(19) Edwards, W. B.; Xu, B.; Akers, W.; Cheney, P. P.; Liang, K.; Rogers, B. E.; Anderson, C. J.; Achilefu, S. Bioconjugate Chem. 2007, 19, 192.

(20) Li, C.; Wang, W.; Wu, Q.; Ke, S.; Houston, J.; Sevick-Muraca, E.; Dong, L.; Chow, D.; Charnsangavej, C.; Gelovani, J. G. Nucl. Med. Biol. 2006, 33, 349.

(21) Gore, J. C.; Manning, H. C.; Quarles, C. C.; Waddell, K. W.; Yankeelov, T. E. Magn. Reson. Imaging 2011, 29, 587.

(22) Weissleder, R.; Pittet, M. J. Nature 2008, 452, 580.

(23) Nolting, D. D.; Gore, J. C.; Pham, W. Curr. Org. Synth. 2011, 8, 521.

(24) Damadian, R. Science 1971, 171, 1151.

(25) Lauterbur, P. C. Nature 1973, 242, 190.

(26) Pekar, J. J. IEEE Eng. Med. Biol. Mag. 2006, 25, 24.

(27) Stapf, S.; Han, S.-I. In NMR Imaging in Chemical Engineering; Stapf, S., Han, S.-I., Eds.; Wiley-VCH Verlag GmbH \& Co. KGaA: 2006, p 1.

(28) Bottrill, M.; Kwok, L.; Long, N. J. Chem. Soc. Rev. 2006, 35, 557.

(29) Caravan, P.; Ellison, J. J.; McMurry, T. J.; Lauffer, R. B. Chem. Rev. 1999, 99, 2293.

(30) Hermann, P.; Kotek, J.; Kubicek, V.; Lukes, I. J. Chem. Soc., Dalton Trans. 2008, 3027.

(31) Reichert, D. E.; Lewis, J. S.; Anderson, C. J. Coord. Chem. Rev. 1999, $184,3$.

(32) Terreno, E.; Castelli, D. D.; Viale, A.; Aime, S. Chem. Rev. 2010, 110, 3019.

(33) Laurent, S.; Forge, D.; Port, M.; Roch, A.; Robic, C.; Vander Elst, L.; Muller, R. N. Chem. Rev. 2008, 108, 2064.

(34) Bulte, J. W. M.; Kraitchman, D. L. NMR Biomed. 2004, 17, 484.

(35) Wang, Y.-X.; Hussain, S.; Krestin, G. Eur. Radiol. 2001, 11, 2319.

(36) Viswanathan, S.; Kovacs, Z.; Green, K. N.; Ratnakar, S. J.; Sherry, A. D. Chem. Rev. 2010, 110, 2960.

(37) Aime, S.; Fasano, M.; Terreno, E. Chem. Soc. Rev. 1998, 27, 19.

(38) Sherry, A. D. J. Less-Common Met. 1989, 149, 133.

(39) Gries, H.; Krause, W., Ed.; Springer Berlin / Heidelberg: 2002; Vol. 221, p 1.

(40) Tóth, É.; Helm, L.; Merbach, A. In Top Curr Chem; Krause, W., Ed.; Springer Berlin / Heidelberg: 2002; Vol. 221, p 61.

(41) Cohen, S. M.; Xu, J.; Radkov, E.; Raymond, K. N.; Botta, M.; Barge, A.; Aime, S. Inorg. Chem. 2000, 39, 5747. 
(42) Datta, A.; Hooker, J. M.; Botta, M.; Francis, M. B.; Aime, S.; Raymond, K. N. J. Am. Chem. Soc. 2008, 130, 2546.

(43) Werner, E. J.; Datta, A.; Jocher, C. J.; Raymond, K. N. Angew. Chem., Int. Ed. 2008, 47, 8568.

(44) Pierre, V. C.; Botta, M.; Aime, S.; Raymond, K. N. J. Am. Chem. Soc. 2006, 128, 5344.

(45) Raymond, K. N.; Pierre, V. C. Bioconjugate Chem. 2004, $16,3$.

(46) Werner, E. J.; Kozhukh, J.; Botta, M.; Moore, E. G.; Avedano, S.; Aime, S.; Raymond, K. N. Inorg. Chem. 2008, 48, 277.

(47) Datta, A.; Raymond, K. N. Acc. Chem. Res. 2009, 42, 938.

(48) Aime, S.; Batsanov, A. S.; Botta, M.; Howard, J. A. K.; Parker, D.; Senanayake, K.; Williams, G. Inorg. Chem. 1994, 33, 4696.

(49) Koenig, S. H.; Brown, R. D. Prog. Nucl. Magn. Reson. Spectrosc. 1990, 22, 487.

(50) Botta, M. Eur. J. Inorg. Chem. 2000, 2000, 399.

(51) Bonnet, C. S.; Fries, P. H.; Crouzy, S.; Sénèque, O.; Cisnetti, F.; Boturyn, D.; Dumy, P.; Delangle, P. Chem. Eur. J. 2009, 15, 7083.

(52) Fulton, D. A.; Elemento, E. M.; Aime, S.; Chaabane, L.; Botta, M.; Parker, D. Chem. Commun. 2006, 1064.

(53) Ali, M. M.; Woods, M.; Caravan, P.; Opina, A. C. L.; Spiller, M.; Fettinger, J. C.; Sherry, A. D. Chem. Eur. J. 2008, 14, 7250.

(54) Aime, S.; Gianolio, E.; Longo, D.; Pagliarin, R.; Lovazzano, C.; Sisti, M. ChemBioChem 2005, 6, 818.

(55) Jaszberenyi, Z.; Sour, A.; Toth, E.; Benmelouka, M.; Merbach, A. E. Dalton Trans. 2005, 2713.

(56) Kotek, J.; Lebdušková, P.; Hermann, P.; Vander Elst, L.; Muller, R. N.; Geraldes, C. F. G. C.; Maschmeyer, T.; Lukeš, I.; Peters, J. A. Chem. Eur. J. 2003, 9, 5899.

(57) Laus, S.; Ruloff, R.; Tóth, É.; Merbach, A. E. Chem. Eur. J. 2003, 9, 3555.

(58) Tei, L.; Botta, M.; Lovazzano, C.; Barge, A.; Milone, L.; Aime, S. Magn. Reson. Chem. 2008, 46, S86.

(59) Tei, L.; Gugliotta, G.; Baranyai, Z.; Botta, M. Dalton Trans. 2009, 9712.

(60) Lebduskova, P.; Hermann, P.; Helm, L.; Toth, E.; Kotek, J.; Binnemans, K.; Rudovsky, J.; Lukes, I.; Merbach, A. E. Dalton Trans. 2007, 493.

(61) Karfeld, L. S.; Bull, S. R.; Davis, N. E.; Meade, T. J.; Barron, A. E. Bioconjugate Chem. 2007, 18, 1697.

(62) Yang, J. J.; Yang, J.; Wei, L.; Zurkiya, O.; Yang, W.; Li, S.; Zou, J.; Zhou, Y.; Maniccia, A. L. W.; Mao, H.; Zhao, F.; Malchow, R.; Zhao, S.; Johnson, J.; Hu, X.; Krogstad, E.; Liu, Z.-R. J. Am. Chem. Soc. 2008, 130, 9260.

(63) Abiraj, K.; Jaccard, H.; Kretzschmar, M.; Helm, L.; Maecke, H. R. Chem. Commun. 2008, 3248.

(64) Botta, M.; Quici, S.; Pozzi, G.; Marzanni, G.; Pagliarin, R.; Barra, S.; Geninatti Crich, S. Org. Biomol. Chem. 2004, 2.

(65) Ratnakar, S. J.; Alexander, V. Eur. J. Inorg. Chem. 2005, 2005, 3918.

(66) Villaraza, A. J.; Bumb, A.; Brechbiel, M. W. Chem. Rev. 2010, 110, 2921. 
(67) Aime, S.; Castelli, D. D.; Crich, S. G.; Gianolio, E.; Terreno, E. Acc. Chem. Res. 2009, 42, 822.

(68) Caravan, P. Chem. Soc. Rev. 2006, 35.

(69) Venditto, V. J.; Regino, C. A. S.; Brechbiel, M. W. Mol. Pharm. 2005, 2, 302.

(70) Kobayashi, H.; Kawamoto, S.; Jo, S.-K.; Bryant, H. L.; Brechbiel, M. W.; Star, R. A. Bioconjugate Chem. 2003, 14, 388.

(71) Wiener, E.; Brechbiel, M. W.; Brothers, H.; Magin, R. L.; Gansow, O. A.; Tomalia, D. A.; Lauterbur, P. C. Magn. Reson. Med. 1994, 31, 1.

(72) Langereis, S.; de Lussanet, Q. G.; van Genderen, M. H. P.; Backes, W. H.; Meijer, E. W. Macromolecules 2004, 37, 3084.

(73) Langereis, S.; Dirksen, A.; Hackeng, T. M.; van Genderen, M. H. P.; Meijer, E. W. New J. Chem. 2007, 31, 1152.

(74) Nwe, K.; Xu, H.; Regino, C. A. S.; Bernardo, M.; lleva, L.; Riffle, L.; Wong, K. J.; Brechbiel, M. W. Bioconjugate Chem. 2009, 20, 1412.

(75) Bryant, L. H.; Brechbiel, M. W.; Wu, C.; Bulte, J. W. M.; Herynek, V.; Frank, J. A. J. Magn. Reson. Imaging 1999, 9, 348.

(76) Aime, S.; Frullano, L.; Geninatti Crich, S. Angew. Chem., Int. Ed. 2002, 41, 1017.

(77) Caravan, P. Acc. Chem. Res. 2009, 42, 851.

(78) Assfalg, M.; Gianolio, E.; Zanzoni, S.; Tomaselli, S.; Lo Russo, V.; Cabella, C.; Ragona, L.; Aime, S.; Molinari, H. J. Med. Chem. 2007, 50, 5257.

(79) Tei, L.; Barge, A.; Geninatti Crich, S.; Pagliarin, R.; Negri, V.; Ramella, D.; Cravotto, G.; Aime, S. Chem. Eur. J. 2010, 16, 8080.

(80) Opsahl, L. R.; Uzgiris, E. E.; Vera, D. R. Acad. Radiol. 1995, 2, 762.

(81) Lucas, R. L.; Benjamin, M.; Reineke, T. M. Bioconjugate Chem. 2007, 19, 24.

(82) Prasuhn, J. D. E.; Yeh, R. M.; Obenaus, A.; Manchester, M.; Finn, M. G. Chem. Commun. 2007, 1269.

(83) Liepold, L. O.; Abedin, M. J.; Buckhouse, E. D.; Frank, J. A.; Young, M. J.; Douglas, T. Nano Lett. 2009, 9, 4520.

(84) Liepold, L.; Anderson, S.; Willits, D.; Oltrogge, L.; Frank, J. A.; Douglas, T.; Young, M. Magn. Reson. Med. 2007, 58, 871.

(85) Datta, A.; Hooker, J. M.; Botta, M.; Francis, M. B.; Aime, S.; Raymond, K. N. J. Am. Chem. Soc. 2008, 130, 2546.

(86) Garimella, P. D.; Datta, A.; Romanini, D. W.; Raymond, K. N.; Francis, M. B. J. Am. Chem. Soc. 2011, 133, 14704.

(87) Hooker, J. M.; Datta, A.; Botta, M.; Raymond, K. N.; Francis, M. B. Nano Lett. 2007, 7, 2207.

(88) Song, Y.; Kohlmeir, E. K.; Meade, T. J. J. Am. Chem. Soc. 2008, 130, 6662.

(89) Sirlin, C. B.; Vera, D. R.; Corbeil, J. A.; Caballero, M. B.; Buxton, R. B.; Mattrey, R. F. Acad. Radiol. 2004, 11, 1361.

(90) Overoye-Chan, K.; Koerner, S.; Looby, R. J.; Kolodziej, A. F.; Zech, S. G.; Deng, Q.; Chasse, J. M.; McMurry, T. J.; Caravan, P. J. Am. Chem. Soc. 2008, 130,6025 . 
(91) Ye, F.; Jeong, E.-K.; Jia, Z.; Yang, T.; Parker, D.; Lu, Z.-R. Bioconjugate Chem. 2008, 19, 2300.

(92) Endres, P. J.; MacRenaris, K. W.; Vogt, S.; Meade, T. J. Bioconjugate Chem. 2008, 19, 2049.

(93) Livramento, J. B.; Tóth, É.; Sour, A.; Borel, A.; Merbach, A. E.; Ruloff, R. Angew. Chem., Int. Ed. 2005, 44, 1480.

(94) Parac-Vogt, T. N.; Vander Elst, L.; Kimpe, K.; Laurent, S.; Burtea, C.; Chen, F.; Van Deun, R.; Ni, Y.; Muller, R. N.; Binnemans, K. Contrast Media Mol. Imaging 2006, 1, 267.

(95) Accardo, A.; Tesauro, D.; Aloj, L.; Pedone, C.; Morelli, G. Coord. Chem. Rev. 2009, 253, 2193.

(96) André, J. P.; Tóth, É.; Fischer, H.; Seelig, A.; Mäcke, H. R.; Merbach, A. E. Chem. Eur. J. 1999, 5, 2977.

(97) Kielar, F.; Tei, L.; Terreno, E.; Botta, M. J. Am. Chem. Soc. 2010, 132, 7836 .

(98) Schuhle, D. T.; Polasek, M.; Lukes, I.; Chauvin, T.; Toth, E.; Schatz, J.; Hanefeld, U.; Stuart, M. C. A.; Peters, J. A. Dalton Trans. 2010, 39, 185.

(99) Torres, S.; Prata, M. I. M.; Santos, A. C.; André, J. P.; Martins, J. A.; Helm, L.; Tóth, É.; García-Martín, M. L.; Rodrigues, T. B.; López-Larrubia, P.; Cerdán, S.; Geraldes, C. F. G. C. NMR Biomed. 2008, 21, 322.

(100) Liu, T.; Qian, Y.; Hu, X.; Ge, Z.; Liu, S. J. Mater. Chem. 2012, 22, 5020.

(101) Jin, T.; Yoshioka, Y.; Fujii, F.; Komai, Y.; Seki, J.; Seiyama, A. Chem. Commun. 2008, 5764.

(102) Mulder, W. J. M.; Koole, R.; Brandwijk, R. J.; Storm, G.; Chin, P. T. K.; Strijkers, G. J.; de Mello Donegá, C.; Nicolay, K.; Griffioen, A. W. Nano Lett. 2005, $6,1$.

(103) Yang, H.; Santra, S.; Walter, G. A.; Holloway, P. H. Adv. Mater. 2006, 18, 2890.

(104) Gerion, D.; Herberg, J.; Bok, R.; Gjersing, E.; Ramon, E.; Maxwell, R.; Kurhanewicz, J.; Budinger, T. F.; Gray, J. W.; Shuman, M. A.; Chen, F. F. J. Phys. Chem. C 2007, 111, 12542.

(105) Manus, L. M.; Mastarone, D. J.; Waters, E. A.; Zhang, X.-Q.; SchultzSikma, E. A.; MacRenaris, K. W.; Ho, D.; Meade, T. J. Nano Lett. 2009, 10, 484.

(106) Feldmann, V.; Engelmann, J.; Gottschalk, S.; Mayer, H. A. J. Colloid Interface Sci. 2012, 366, 70.

(107) Vivero-Escoto, J. L.; Taylor-Pashow, K. M. L.; Huxford, R. C.; Della Rocca, J.; Okoruwa, C.; An, H.; Lin, W.; Lin, W. Small 2011, 7, 3519.

(108) Voisin, P.; Ribot, E. J.; Miraux, S.; Bouzier-Sore, A. K.; Lahitte, J. F.; Bouchaud, V.; Mornet, S.; Thiaudiere, E.; Franconi, J. M.; Raison, L.; Labrugere, C.; Delville, M. H. Bioconjugate Chem. 2007, 18, 1053.

(109) Taylor, K. M. L.; Kim, J. S.; Rieter, W. J.; An, H.; Lin, W.; Lin, W. J. Am. Chem. Soc. 2008, 130, 2154.

(110) Plush, S. E.; Woods, M.; Zhou, Y.-F.; Kadali, S. B.; Wong, M. S.; Sherry, A. D. J. Am. Chem. Soc. 2009, 131, 15918.

(111) Richard, C.; Doan, B. T.; Beloeil, J. C.; Bessodes, M.; Toth, E.; Scherman, D. Nano Lett. 2008, 8, 232. 
(112) Matson, M. L.; Wilson, L. J. Future Med. Chem. 2010, 2, 491.

(113) Ananta, J. S.; Wilson, L. J. In Chemistry of Nanocarbons; Akasaka, T., Wudl, F., Nagase, S., Eds.; John Wiley \& Sons, Ltd: 2010, p 287.

(114) Alric, C.; Taleb, J.; Duc, G. L.; Mandon, C.; Billotey, C.; Meur-Herland, A. L.; Brochard, T.; Vocanson, F.; Janier, M.; Perriat, P.; Roux, S.; Tillement, O. J. Am. Chem. Soc. 2008, 130, 5908.

(115) Debouttière, P. J.; Roux, S.; Vocanson, F.; Billotey, C.; Beuf, O.; FavreRéguillon, A.; Lin, Y.; Pellet-Rostaing, S.; Lamartine, R.; Perriat, P.; Tillement, O. Adv. Funct. Mater. 2006, 16, 2330.

(116) Moriggi, L. C.; Cannizzo, C.; Dumas, E.; Mayer, C. d. R.; Ulianov, A.; Helm, L. J. Am. Chem. Soc. 2009, 131, 10828.

(117) Park, J.-A.; Reddy, P. A. N.; Kim, H.-K.; Kim, I.-S.; Kim, G.-C.; Chang, Y.; Kim, T.-J. Bioorg. Med. Chem. Lett. 2008, 18, 6135.

(118) Warsi, M. F.; Chechik, V. Phys. Chem. Chem. Phys. 2011, 13, 9812.

(119) Liu, L.; Ding, H.; Yong, K.-T.; Roy, I.; Law, W.-C.; Kopwitthaya, A.; Kumar, R.; Erogbogbo, F.; Zhang, X.; Prasad, P. Plasmonics 2011, 6, 105.

(120) Sun, H.; Yuan, Q.; Zhang, B.; Ai, K.; Zhang, P.; Lu, L. Nanoscale 2011, 3, 1990.

(121) Ntziachristos, V. Nat. Meth. 2010, 7, 603.

(122) Wu, J. C.; Sundaresan, G.; Iyer, M.; Gambhir, S. S. Mol. Ther. 2001, 4, 297.

(123) Choy, G.; Choyke, P.; Libutti, S. K. Mol. Imaging 2003, 2, 303.

(124) Villalobos, V.; Naik, S.; Piwnica-Worms, D. Annu. Rev. Biomed. Eng. 2007, 9,321 .

(125) Thorne, S. H.; Contag, C. H. P. IEEE 2005, 93, 750.

(126) Jobsis, F. Science 1977, 198, 1264.

(127) Licha, K. In Top Curr Chem; Krause, W., Ed.; Springer Berlin / Heidelberg: 2002; Vol. 222, p 1.

(128) Sevick-Muraca, E. M.; Houston, J. P.; Gurfinkel, M. Curr. Opin. Chem. Biol. 2002, 6, 642 .

(129) Faulkner, S.; Pope, S. J. A.; Burton-Pye, B. P. Appl. Spectrosc. Rev. 2005, 40,1

(130) Terai, T.; Kikuchi, K.; Iwasawa, S.; Kawabe, T.; Hirata, Y.; Urano, Y.; Nagano, T. J. Am. Chem. Soc. 2006, 128, 8699.

(131) Bunzli, J.-C. G.; Piguet, C. Chem. Soc. Rev. 2005, 34, 1048.

(132) Beeby, A.; Bushby, L. M.; Maffeo, D.; Gareth Williams, J. A. J. Chem. Soc., Dalton Trans. 2002, 48.

(133) Faulkner, S.; Carrie, M.-C.; Pope, S. J. A.; Squire, J.; Beeby, A.; Sammes, P. G. J. Chem. Soc., Dalton Trans. 2004, 1405.

(134) Pal, R.; Parker, D. Org. Biomol. Chem. 2008, 6.

(135) Burton-Pye, B. P.; Heath, S. L.; Faulkner, S. J. Chem. Soc., Dalton Trans. 2005, 146.

(136) Koullourou, T.; Natrajan, L. S.; Bhavsar, H.; Pope, S. J. A.; Feng, J.; Narvainen, J.; Shaw, R.; Scales, E.; Kauppinen, R.; Kenwright, A. M.; Faulkner, S. J. Am. Chem. Soc. 2008, 130, 2178. 
(137) Imbert, D.; Cantuel, M.; Bünzli, J.-C. G.; Bernardinelli, G.; Piguet, C. J. Am. Chem. Soc. 2003, 125, 15698.

(138) Klink, S. I.; Keizer, H.; Veggel, F. C. J. M., van Angew. Chem., Int. Ed. 2000, 39, 4319.

(139) Pandya, S.; Yu, J.; Parker, D. J. Chem. Soc., Dalton Trans. 2006, 2757.

(140) Montgomery, C. P.; Murray, B. S.; New, E. J.; Pal, R.; Parker, D. Acc. Chem. Res. 2009, 42, 925.

(141) Bünzli, J.-C. G. Chem. Rev. 2010, 110, 2729.

(142) Beeby, A.; S. Dickins, R.; Faulkner, S.; Parker, D.; A. Gareth Williams, J. Chem. Commun. 1997, 1401.

(143) Werts, M. H. V.; Woudenberg, R. H.; Emmerink, P. G.; van Gassel, R.; Hofstraat, J. W.; Verhoeven, J. W. Angew. Chem., Int. Ed. 2000, 39, 4542.

(144) Bünzli, J.-C. G. Acc. Chem. Res. 2005, 39, 53.

(145) Smith, A. M.; Gao, X.; Nie, S. Photochem. Photobiol. 2004, 80, 377.

(146) Xing, Y. In Nanoplatform-Based Molecular Imaging; Chen, X., Ed.; John Wiley \& Sons, Inc.: 2011, p 159.

(147) Diez, I.; Ras, R. H. A. Nanoscale 2011, 3, 1963.

(148) Patel, S. A.; Richards, C. I.; Hsiang, J.-C.; Dickson, R. M. J. Am. Chem. Soc. 2008, 130, 11602.

(149) Xu, H.; Suslick, K. S. Adv. Mater. 2010, 22, 1078.

(150) Zheng, J.; Dickson, R. M. J. Am. Chem. Soc. 2002, 124, 13982.

(151) Petty, J. T.; Zheng, J.; Hud, N. V.; Dickson, R. M. J. Am. Chem. Soc. 2004, $126,5207$.

(152) Richards, C. I.; Choi, S.; Hsiang, J.-C.; Antoku, Y.; Vosch, T.; Bongiorno, A.; Tzeng, Y.-L.; Dickson, R. M. J. Am. Chem. Soc. 2008, 130, 5038.

(153) Vosch, T.; Antoku, Y.; Hsiang, J.-C.; Richards, C. I.; Gonzalez, J. I.; Dickson, R. M. Proc. Natl. Acad. Sci. U. S. A. 2007, 104, 12616.

(154) Yu, J.; Choi, S.; Richards, C. I.; Antoku, Y.; Dickson, R. M. Photochem. Photobiol. 2008, 84, 1435.

(155) Yu, J.; Patel, S. A.; Dickson, R. M. Angew. Chem., Int. Ed. 2007, 46, 2028.

(156) Yu, J.; Choi, S.; Dickson, R. M. Angew. Chem., Int. Ed. 2009, 48, 318.

(157) Pellegatti, L.; Zhang, J.; Drahos, B.; Villette, S.; Suzenet, F.; Guillaumet, G.; Petoud, S.; Toth, E. Chem. Commun. 2008, 6591.

(158) Mamedov, I.; Parac-Vogt, T. N.; Logothetis, N. K.; Angelovski, G. Dalton Trans. 2010, 39, 5721.

(159) Mindt, T. L.; Müller, C.; Stuker, F.; Salazar, J.-F. d. r.; Hohn, A.; Mueggler, T.; Rudin, M.; Schibli, R. Bioconjugate Chem. 2009, 20, 1940.

(160) Manning, H. C.; Goebel, T.; Thompson, R. C.; Price, R. R.; Lee, H.; Bornhop, D. J. Bioconjugate Chem. 2004, 15, 1488.

(161) Li, H.; Gray, B. D.; Corbin, I.; Lebherz, C.; Choi, H.; Lund-Katz, S.; Wilson, J. M.; Glickson, J. D.; Zhou, R. Acad. Radiol. 2004, 11, 1251.

(162) Tekade, R. K.; Kumar, P. V.; Jain, N. K. Chem. Rev. 2009, 110, 2574.

(163) Bosman, A. W.; Janssen, H. M.; Meijer, E. W. Chem. Rev. 1999, 99, 1665.

(164) Menjoge, A. R.; Kannan, R. M.; Tomalia, D. A. Drug Discov. Today 2010, $15,171$. 
(165) Talanov, V. S.; Regino, C. A. S.; Kobayashi, H.; Bernardo, M.; Choyke, P. L.; Brechbiel, M. W. Nano Lett. 2006, 6, 1459.

(166) Xu, H.; Regino, C. A. S.; Koyama, Y.; Hama, Y.; Gunn, A. J.; Bernardo, M.; Kobayashi, H.; Choyke, P. L.; Brechbiel, M. W. Bioconjugate Chem. 2007, 18, 1474.

(167) Boswell, C. A.; Eck, P. K.; Regino, C. A. S.; Bernardo, M.; Wong, K. J.; Milenic, D. E.; Choyke, P. L.; Brechbiel, M. W. Mol. Pharm. 2008, 5, 527.

(168) Barrett, T.; Choyke, P. L.; Kobayashi, H. Contrast Media Mol. Imaging 2006, 1, 230.

(169) Mulder, W. J. M.; Strijkers, G. J.; van Tilborg, G. A. F.; Griffioen, A. W.; Nicolay, K. NMR Biomed. 2006, 19, 142.

(170) Mulder, W. J. M.; Strijkers, G. J.; Griffioen, A. W.; van Bloois, L.; Molema, G.; Storm, G.; Koning, G. A.; Nicolay, K. Bioconjugate Chem. 2004, 15, 799.

(171) Kamaly, N.; Kalber, T.; Ahmad, A.; Oliver, M. H.; So, P.-W.; Herlihy, A. H.; Bell, J. D.; Jorgensen, M. R.; Miller, A. D. Bioconjugate Chem. 2008, 19, 118.

(172) Mulder, W. J. M.; Strijkers, G. J.; Briley-Saboe, K. C.; Frias, J. C.; Aguinaldo, J. G. S.; Vucic, E.; Amirbekian, V.; Tang, C.; Chin, P. T. K.; Nicolay, K.; Fayad, Z. A. Magn. Reson. Med. 2007, 58, 1164.

(173) Moriggi, L.; Aebischer, A.; Cannizzo, C.; Sour, A.; Borel, A.; Bunzli, J.-C. G.; Helm, L. Dalton Trans. 2009, 2088.

(174) Dehaen, G.; Eliseeva, S. V.; Kimpe, K.; Laurent, S.; Vander Elst, L.; Muller, R. N.; Dehaen, W.; Binnemans, K.; Parac-Vogt, T. N. Chem. Eur. J. 2012, 18, 293.

(175) SalmanOgli, A. Cancer Nanotechnology 2011, 2, 1.

(176) Mulder, W. J. M.; Strijkers, G. J.; van Tilborg, G. A. F.; Cormode, D. P.; Fayad, Z. A.; Nicolay, K. Acc. Chem. Res. 2009, 42, 904.

(177) Mulder, W. J. M.; Koole, R.; Brandwijk, R. J.; Storm, G.; Chin, P. T. K.; Strijkers, G. J.; deMelloDonega, C.; Nicolay, K.; Griffioen, A. W. Nano Lett. 2006, 6, 1.

(178) van Tilborg, G. A. F.; Mulder, W. J. M.; Chin, P. T. K.; Storm, G.; Reutelingsperger, C. P.; Nicolay, K.; Strijkers, G. J. Bioconjugate Chem. 2006, 17, 865.

(179) Prinzen, L.; Miserus, R.-J. J. H. M.; Dirksen, A.; Hackeng, T. M.; Deckers, N.; Bitsch, N. J.; Megens; Douma, K.; Heemskerk, J. W.; Kooi, M. E.; Frederik, P. M.; Slaaf, D. W.; van Zandvoort, M. A. M. J.; Reutelingsperger, C. P. M. Nano Lett. 2006, 7, 93.

(180) Bakalova, R.; Zhelev, Z.; Aoki, I.; Masamoto, K.; Mileva, M.; Obata, T.; Higuchi, M.; Gadjeva, V.; Kanno, I. Bioconjugate Chem. 2008, $19,1135$.

(181) Park, J.; Bhuniya, S.; Lee, H.; Noh, Y.-W.; Lim, Y. T.; Jung, J. H.; Hong, K. S.; Kim, J. S. Chem. Commun. 2012, 48, 3218.

(182) Song, Y.; Xu, X.; MacRenaris, K. W.; Zhang, X.-Q.; Mirkin, C. A.; Meade, T. J. Angew. Chem., Int. Ed. 2009, 121, 9143.

(183) Pinho, S. L. C.; Faneca, H.; Geraldes, C. F. G. C.; Delville, M.-H.; Carlos, L. D.; Rocha, J. Biomaterials 2012, 33, 925.

(184) Pinho, S. L. C.; Faneca, H.; Geraldes, C. F. G. C.; Rocha, J.; Carlos, L. D.; Delville, M.-H. Eur. J. Inorg. Chem. 2012, 2828. 
(185) Rieter, W. J.; Kim, J. S.; Taylor, K. M. L.; An, H.; Lin, W.; Tarrant, T.; Lin, W. Angew. Chem., Int. Ed. 2007, 46, 3680.

(186) Nam, T.; Park, S.; Lee, S.-Y.; Park, K.; Choi, K.; Song, I. C.; Han, M. H.; Leary, J. J.; Yuk, S. A.; Kwon, I. C.; Kim, K.; Jeong, S. Y. Bioconjugate Chem. 2010, 21, 578.

(187) Tan, W. B.; Zhang, Y. J. Nanosci. Nanotechnol. 2007, 7, 2389.

(188) Anderson, E. A.; Isaacman, S.; Peabody, D. S.; Wang, E. Y.; Canary, J. W.; Kirshenbaum, K. Nano Lett. 2006, 6, 1160.

(189) Suci, P. A.; Berglund, D. L.; Liepold, L.; Brumfield, S.; Pitts, B.; Davison, W.; Oltrogge, L.; Hoyt, K. O.; Codd, S.; Stewart, P. S.; Young, M.; Douglas, T. Chem. Biol. 2007, 14, 387.

(190) Pokorski, J. K.; Breitenkamp, K.; Liepold, L. O.; Qazi, S.; Finn, M. G. J. Am. Chem. Soc. 2011, 133, 9242.

(191) Tsotsalas, M.; Busby, M.; Gianolio, E.; Aime, S.; De Cola, L. Chem. Mater. 2008, 20, 5888. 
"You're never too old, never too bad, never too late and never too sick to start from the scratch once again" Bikram Choudhury

\section{CHAPTER 3}

\section{MULTIFUNCTIONAL HYBRID SILVER NANOSTRUCTURES ( $h$ AgNSS) GROWN USING A POLYAMINO CARBOXYLIC ACID SCAFFOLD}

\section{Abstract}

In this chapter, the aqueous-based, one-step synthesis of hybrid silver nanostructures (hAgNSs) is described. hAgNSs were grown using a polyamino carboxylic acid scaffold, namely 1,4,7,10-tetraaza-1-(1-aminobutyl)-4,7,10triacetic acid cyclododecane (AB-DO3A). While the carboxylic moieties of the ABDO3A scaffold provide nucleation sites for nanoparticle (NP) growth and contribute to the further stabilization of the species formed, the amine group provides both water solubility and an active group (for further conjugation) to the final product. A feasible arrangement for these $h$ AgNSs is proposed based on UVVis absorbance spectroscopy, transmission electron microscopy, dynamic light scattering, fluorescence (lifetime and steady-state), NMR, infrared spectroscopy and mass spectrometry. The engineered $h A g N S s$ consist of two functional units: a "large" AgNP stabilized by the carboxylate moieties of the AB-DO3A scaffold, that can act simultaneously as a template for surface modification and potentially as a therapeutic agent, and "smaller" Ag nanoclusters (AgNCs) that can be used as optical trackers. 


\subsection{Introduction}

Noble metal nanoparticles (NPs), known for their chameleon-like assets, are interesting for solar energy cells ${ }^{1,2}$ and biological $\left.\right|^{3-5}$ applications. Due to the large surface areas they offer, in which virtually any functional probe can be integrated via surface modification, ${ }^{6}$ noble metal NPs have found several applications in both therapeutics and optical diagnostics. ${ }^{4-6}$ Their optical properties, characterized by strong light scattering and absorption due to localized surface plasmon resonance, are strongly dependent on their shape and size..$^{7-9}$

Silver NPs (AgNPs, Ag > 100 atoms) are characterized by their antiviral, antibacterial and antifungal properties as well as their applications for surface enhanced Raman scattering (SERS) and metal enhanced fluorescence (MEF). ${ }^{10,11}$ While the use of AgNPs in cancer therapy is still limited, these nanostructures are known to inhibit cell proliferation in human cancer cells. ${ }^{12,13}$ In contrast, noble metal nanoclusters (NCs), containing up to only 100 atoms, ${ }^{14,15}$ show very different behavior than their larger NP analogues. In particular, silver NCs $\left(\mathrm{Ag}_{\mathrm{n}} \mathrm{NCs}\right)$, consisting of a limited number of silver atoms $(n \sim 2-10)$ exhibit fluorescence ${ }^{16}$, electro(chemi)luminescence ${ }^{17}$ and two-photon fluorescence; ${ }^{18}$ properties that are highly appealing for medical applications.

Despite several fairly established synthetic routes for the fabrication of AgNPs, ${ }^{3,8,11}$ the need for synthetic protocols that contribute to a more controlled fabrication of these nanostructured materials leaves space for improvement. In this chapter, the templating effect of a polyamine carboxylic acid scaffold, namely 1-(4-aminobutyl)-4,7,10tris(carboxymethyl)-1,4,7,10-tetraazacyclododecane (AB-DO3A, Figure 3.1A), on the formation of AgNPs is investigated. The formation of AgNPs 
of nanometer dimensions is triggered by a single-step, aqueous, chemical reduction of $\mathrm{AgNO}_{3}$ with $\mathrm{NaBH}_{4}$. During and after formation, $\mathrm{AB}-\mathrm{DO} 3 \mathrm{~A}$ can stabilize the AgNPs and, surprisingly, this also leads to the concomitant formation of fluorescent $\mathrm{Ag}_{\mathrm{n}} \mathrm{NCs}$. The hybrid silver nanostructures (hAgNSs) formed consist of AgNPs presumably stabilized by the cyclen core and the carboxylic moieties of the pendant arms of the AB-DO3A scaffold where, also, $\mathrm{Ag}_{\mathrm{n}} \mathrm{NCs}$ have grown (Figure 3.1B).

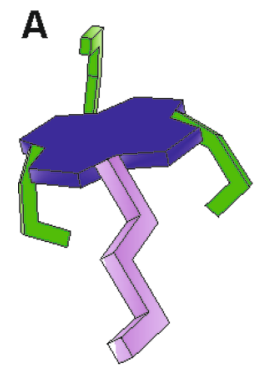

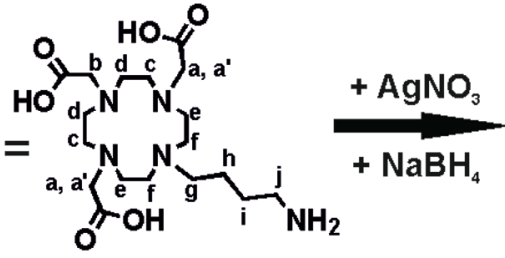

AB-DO3A

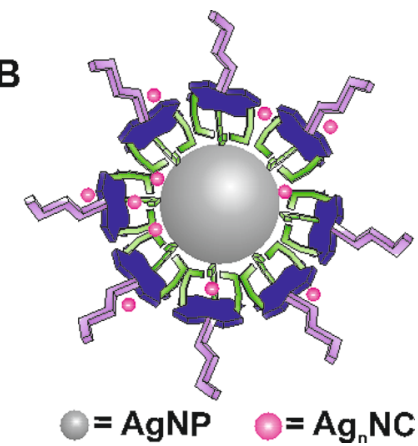

Figure 3.1. Hybrid silver nanostructures (hAgNSs) formed by the chemical reduction of $\mathrm{AgNO}_{3}$ in the presence of the 1,4,7,10-tetraaza-1-(1-aminobutyl)-4,7,10-triacetic acid cyclododecane (AB-DO3A) scaffold. The localization of the AgNCs within the AgNP is only a schematic representation.

\subsection{Design and Synthesis}

Several stabilizing templates/scaffolds have been used for the fabrication of AgNPs, including peptides, ${ }^{19}$ aminated $\beta$-cyclodextrins, ${ }^{20}$ polymers, ${ }^{21-24}$ macrocycles such as cucurbituril ${ }^{12}$ and dendrimers. ${ }^{25,26}$ The various reducing agents used, e.g., citrate, ascorbic acid and sodium borohydride ${ }^{3}$ illustrate the extensive work that has been done in this particular field. Moreover, following similar procedures together with scaffolds such as dendrimers, ${ }^{27}$ oligonucleotides ${ }^{28-30}$ polymers $^{31-34}$ and small organosulfur compounds, ${ }^{35-38}$ fluorescent AgNCs have been 
successfully synthesized. Despite the wide variety of wet chemical approaches described for the synthesis of AgNPs, ${ }^{3,16}$ there is need for new synthetic methodologies that can, hopefully, contribute to a more controlled fabrication of these nanostructures.

Here, it is expected that the multi-functional AB-DO3A scaffold provides the initial nucleation sites for NP growth by means of structurally flexible acarboxylic moieties, known to show strong affinity towards $\mathrm{Ag}^{+}$ions and previously used for the synthesis of AgNCs in "macromolecular" templates.31,32,34 Moreover, the highly polar carboxylic moieties of the acetate pendant arms, as well as the aminobutyl pendant arm, provide water solubility to the scaffold, a vital feature for biological applications. The AB-DO3A scaffold is an interesting, multifunctional platform as other metal functionalities can be included by complexing inside the azacycle and its aminobutyl pendant arm can be used for further functionalization via amide formation.

\subsubsection{Synthesis of the Polyamino Carboxylic Acid Scaffold AB-DO3A}

The synthesis of the polyamino carboxylic acid scaffold 1,4,7,10-tetraaza1-(4-aminobutyl)-4,7,10-triacetic acid cyclododecane (AB-DO3A, 5), is described in Scheme 3.1. First, 1,4,7,10-tetraaza-1-(4-butyl phthalimide)4,7,10-tris(tert-butoxycarboxymethyl)cyclododecane (3) was obtained by alkylation of 1,4,7-tris(tert-butoxycarboxymethyl)-1,4,7,10tetraazacyclododecane (1) with $\mathrm{N}$-(4-bromobutyl)phthalimide (2) in dry acetonitrile using $\mathrm{K}_{2} \mathrm{CO}_{3}$ (under $\mathrm{N}_{2}$ ) for two days at $50^{\circ} \mathrm{C}$. The $\mathrm{N}$-alkylated phthalimide in (3) was cleaved in ethanol using hydrazine. The AB-DO3A ligand (5) was obtained by means of Boc-deprotection of (4) using TFA $/ \mathrm{CH}_{2} \mathrm{Cl}_{2}$ at room temperature overnight. The final product was obtained with an overall yield (after purification) of $37 \%$. 


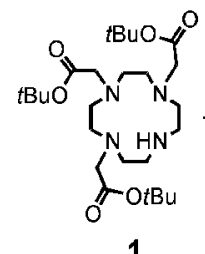<smiles>O=C1c2ccccc2C(=O)N1CCCCBr</smiles>

2

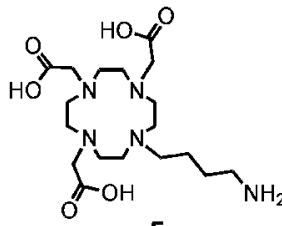

5
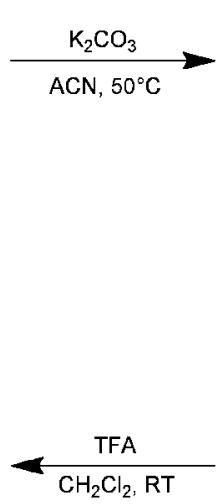

$\mathrm{H}_{2}$

(1)
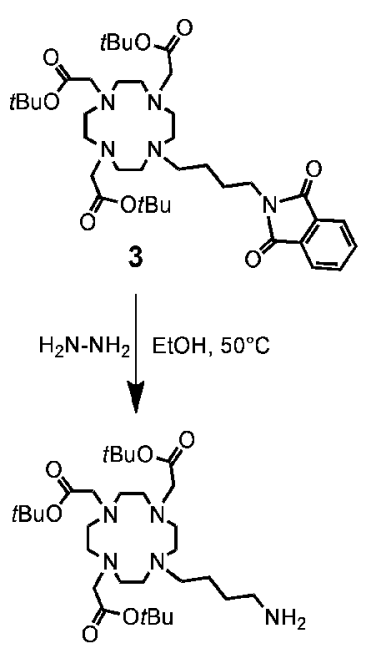

4

Scheme 3.1. Synthetic route followed for the synthesis of AB-DO3A (5).

\subsubsection{Synthesis of hAgNSS.}

hAgNSs were grown by adding $50 \mu \mathrm{L}$ of $\mathrm{AgNO}_{3}$ in water (from a 0.01, 0.02 or $0.03 \mathrm{M}$ stock solution, depending on the desired stoichiometry) to a 4.9 $\mathrm{mL}$ solution of $\mathrm{AB}-\mathrm{DO} 3 \mathrm{~A}$ (final concentration of $\mathrm{AB}-\mathrm{DO} 3 \mathrm{~A}$ is $1 \mathrm{mM}$ ) in water. The solution was allowed to stir for 30 minutes before $50 \mu \mathrm{L}$ of $\mathrm{NaBH}_{4}$ in water (from a $0.01,0.02$ and $0.03 \mathrm{M}$ stock solution, depending on the desired stoichiometry) were slowly added. The solution was stirred for 2 minutes and subsequently left to stand, protected from light, for $24 \mathrm{~h}$. All hAgNS solutions were analyzed and characterized without further purification.

\subsection{Results and Discussion}

\subsubsection{Spectroscopic Characterization of AgNPS}

In order to confirm the formation of AgNPs, a series of control reactions were performed. In the absence of the scaffold and upon addition of 
$\mathrm{NaBH}_{4}$, the solution became turbid, with almost no color (Figure 3.2A, left) and an absorption peak with a maximum at $388 \mathrm{~nm}$ was observed (Figure 3.2B, black line). This absorption band in attributed to AgNPs of various sizes (> $75 \mathrm{~nm}$, whose extinction is dominated by scattering rather than by absorption), ${ }^{39,40}$ that have grown uncontrollably due to the absence of a "stabilizing" agent. By comparison, in the absence of $\mathrm{NaBH}_{4}$ the solution is colorless (Figure 3.2A, right) and a relatively weak UV absorption is observed (Figure 3.2B, grey line).

A

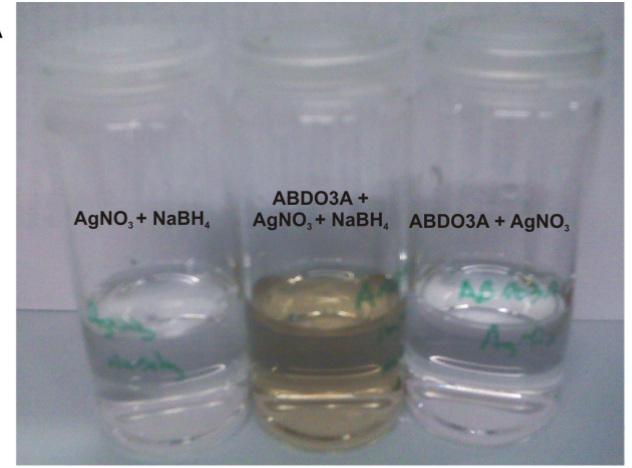

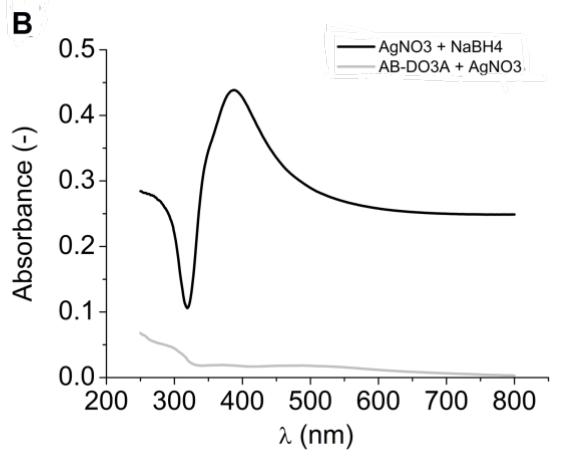

Figure 3.2. A) Image of the solutions of $0.2 \mathrm{mM} \mathrm{AgNO}_{3} / 0.2 \mathrm{mM} \mathrm{NaBH}$ (left), $1 \mathrm{mM}$

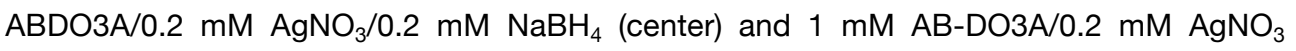
(right) after $24 \mathrm{~h}$. B) UV-Vis spectra of the solutions of $0.2 \mathrm{mM} \mathrm{AgNO}_{3} / 0.2 \mathrm{mM} \mathrm{NaBH}$

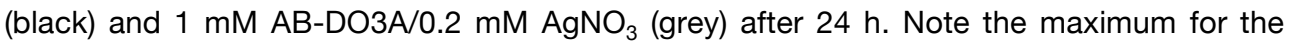
former solution at $388 \mathrm{~nm}$.

In order to guarantee colloidal stability of the NPs formed over time, the growth of AgNPs in the presence of the AB-DO3A scaffold was studied using a five-fold excess of ligand. The stabilizing effect of the AB-DO3A scaffold is demonstrated in Figure 3.2A (center), as the solution becomes visibly yellow upon addition of $\mathrm{NaBH}_{4}$, strongly suggesting the formation of AgNPs of a defined size. In Figure 3.3, the UV spectra of three solutions with different $\mathrm{AB}-\mathrm{DO} 3 \mathrm{~A}: \mathrm{AgNO}_{3}$ ratios, $24 \mathrm{~h}$ after being chemically reduced, 
are presented. Upon reduction, an absorption peak at $402 \mathrm{~nm}$ appears with increasing intensity as the $\mathrm{AB}-\mathrm{DO} 3 \mathrm{~A}: \mathrm{AgNO}_{3}$ ratio decreases. Previously synthesized AgNPs (< $10 \mathrm{~nm}$ ) using pseudo-dendritic polymers $^{41}$ and surfactants ${ }^{42}$ show strong absorption bands around 400 $\mathrm{nm}$. Hence, we attribute this plasmonic absorption to the formation of AgNPs stabilized by the AB-DO3A scaffold. Remarkably, for the 10:1 ABDO3A: $\mathrm{AgNO}_{3}$ solution, a broad shoulder towards the red region of the spectrum is observed (inset Figure 3.3). This peak, which becomes less evident as the relative $\mathrm{AgNO}_{3}$ amount increases, can either point to a less monodisperse sample where larger Ag aggregates are formed or it can originate from other species formed during or after reduction. The position of the shoulder in the UV/Vis spectrum (> $500 \mathrm{~nm}$ ) might indicate the formation of $\mathrm{Ag}_{n} \mathrm{NCs}(\mathrm{n}=2-4)$, known for their electronic transitions between 420 and $520 \mathrm{~nm}$. 17,27,28

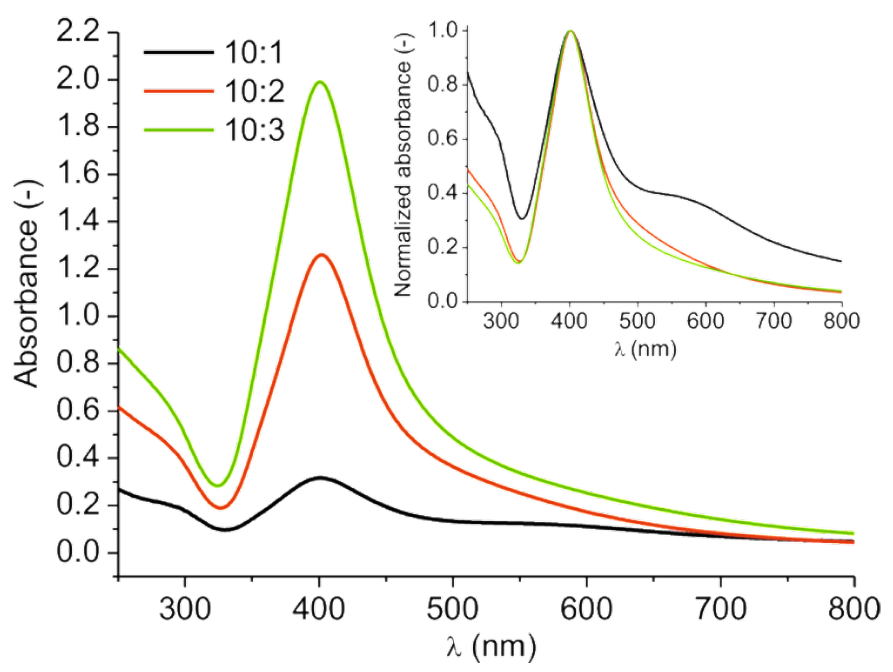

Figure 3.3. UV-Vis spectra of $h A g N S s$ prepared using stoichiometries 10:1, 10:2 and 10:3 $\left(\mathrm{AB}-\mathrm{DO} 3 \mathrm{~A}: \mathrm{AgNO}_{3}\right.$ ) after $24 \mathrm{~h}$. Inset: normalized spectra. 


\subsubsection{AgNP Size Characterization}

The size distribution of the AgNPs formed was measured using two techniques: dynamic light scattering (DLS) and transmission electron microscopy (TEM). $24 \mathrm{~h}$ After reduction of a 10:2 AB-DO3A:AgNO solution, DLS measurements showed that the AgNPs formed had an average size of $3.2 \pm 0.7 \mathrm{~nm}$ (Figure 3.4A). This size is comparable to previously reported AgNPs synthesized using pseudo-dendritic polymers, ${ }^{41}$ with sizes between 2.6 and $4.2 \mathrm{~nm}$ and strong absorption bands around $400 \mathrm{~nm}$. A smaller average size, namely $2.3 \pm 0.7 \mathrm{~nm}$ (Figure 3.4B), was observed by TEM analysis and energy-dispersive X-ray spectroscopy (EDX) analysis showed that these NPs are composed of metallic silver. The difference in measured average size between DLS and TEM $(\sim 1 \mathrm{~nm})$ is attributed to the nature of the scattering measured by each technique. While TEM images illustrate the electron scattering caused by the AgNP metal core only, DLS measurements are based on light scattering caused by spheres of a certain hydrodynamic radius (with solvent contributions included).
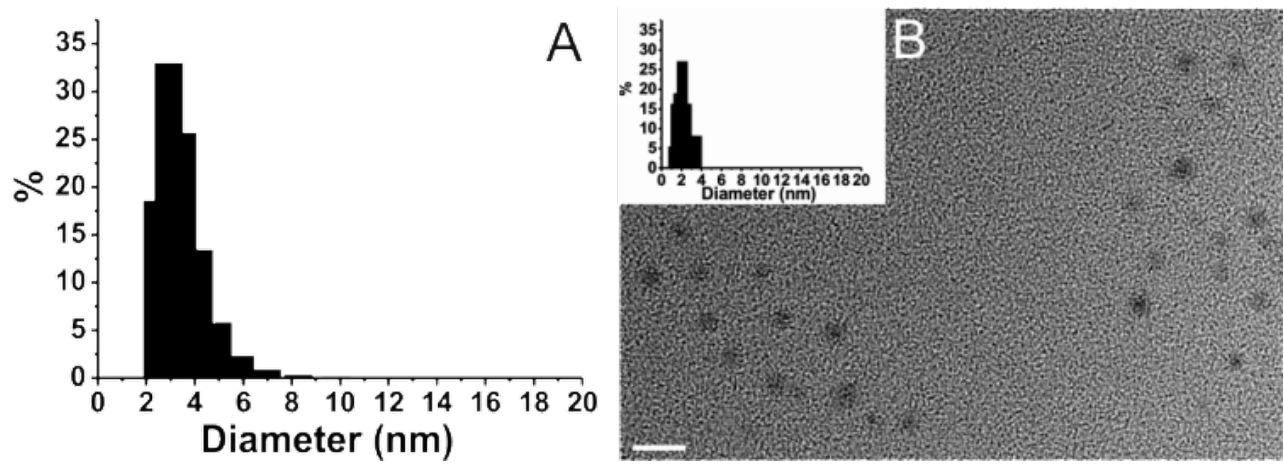

Figure 3.4. Characterization of the AgNP size: A) DLS data of a $10: 2 \mathrm{AB}-\mathrm{DO} 3 \mathrm{~A}: \mathrm{AgNO}_{3}$ sample (after $24 \mathrm{~h}$ ) showing an average particle size of $3.2 \pm 0.7 \mathrm{~nm}$ (SD). B) TE micrograph of the same sample (Scale bar $=10 \mathrm{~nm}$ ). Inset: corresponding histogram showing an average particle size of $2.3 \pm 0.7 \mathrm{~nm}(\mathrm{SD})$. 


\subsubsection{Characterization of $A g_{n} N C S$}

Whilst the UV absorbance at $\sim 400 \mathrm{~nm}$ has been attributed to AgNPs successfully observed by TEM, the shoulder at > $500 \mathrm{~nm}$ strongly suggests the formation of smaller species such as $\mathrm{Ag}_{n} \mathrm{NCs}$. However, it is rather difficult to confirm any $\mathrm{Ag}_{\mathrm{n}} \mathrm{NC}$ absorption due to the strong plasmon resonance peak coming from the AgNPs (which overshadows the signals of the smaller $\left.\mathrm{Ag}_{\mathrm{n}} \mathrm{NCs}\right)^{40,43}$ and the sub-nanometric size of $\mathrm{Ag}_{\mathrm{n}} \mathrm{NCs}$, which is hard to visualize by TEM. Contrary to larger AgNPs, small $\mathrm{Ag}_{n} \mathrm{NCs}$ are known to be fluorescent, ${ }^{16,27,44-46}$ with clear differences in emission spectra when differing in number by only one single atom. ${ }^{28,30}$ Therefore, one way to determine the presence of $\mathrm{Ag}_{\mathrm{n}} \mathrm{NCs}$ is by identifying their (possible) fluorescence emission upon excitation. In order to do so, the emission properties of all three (reduced) solutions of $\mathrm{AB}-\mathrm{DO} 3 \mathrm{~A}: \mathrm{AgNO}_{3}$ with ratios $10: 1,10: 2$ and 10:3 were compared, exciting in the range $\lambda_{\mathrm{exc}}=400$ to 600 $\mathrm{nm}$. As depicted in Figure 3.5, fluorescence emission spectra at 595, 635 and $670 \mathrm{~nm}$, when exciting at 440, 480 and $520 \mathrm{~nm}$ respectively, is observed. Moreover, the maximum fluorescence emission for the 10:1 solution (Figure 3.5A) is observed at $595 \mathrm{~nm}$, for the 10:2 solution (Figure $3.5 \mathrm{~B}$ ) at $635 \mathrm{~nm}$ and for the 10:3 solution (Figure 3.5C) at $670 \mathrm{~nm}$. None of these emission maxima were observed in either the $\mathrm{AgNO}_{3}$ solution nor in the AB-DO3A scaffold solution (Figure 3.10, Appendix 3.1). While emission of $\mathrm{Ag}_{\mathrm{n}} \mathrm{NCs}$ has been previously identified in the regions $610-650 \mathrm{~nm}$ $(n=2,3,5),{ }^{17} 650 \mathrm{~nm}(n=4,5),{ }^{38}$ and $530-650 \mathrm{~nm}(n=1-4),{ }^{27}$ the differences in fluorescence intensity observed for each $\lambda_{\mathrm{exc}}$ show that, as the ABDO3A:AgNO3 ratio increases, the emission maximum shifts to the red. One possible explanation for the difference in emission intensities could come from the interaction with the organic scaffold, as it has been previously observed that the absorption and emission properties of few- 
atom $\mathrm{Ag}_{\mathrm{n}} \mathrm{NCs}$ change dramatically according to the chemical environment surrounding them. ${ }^{17}$
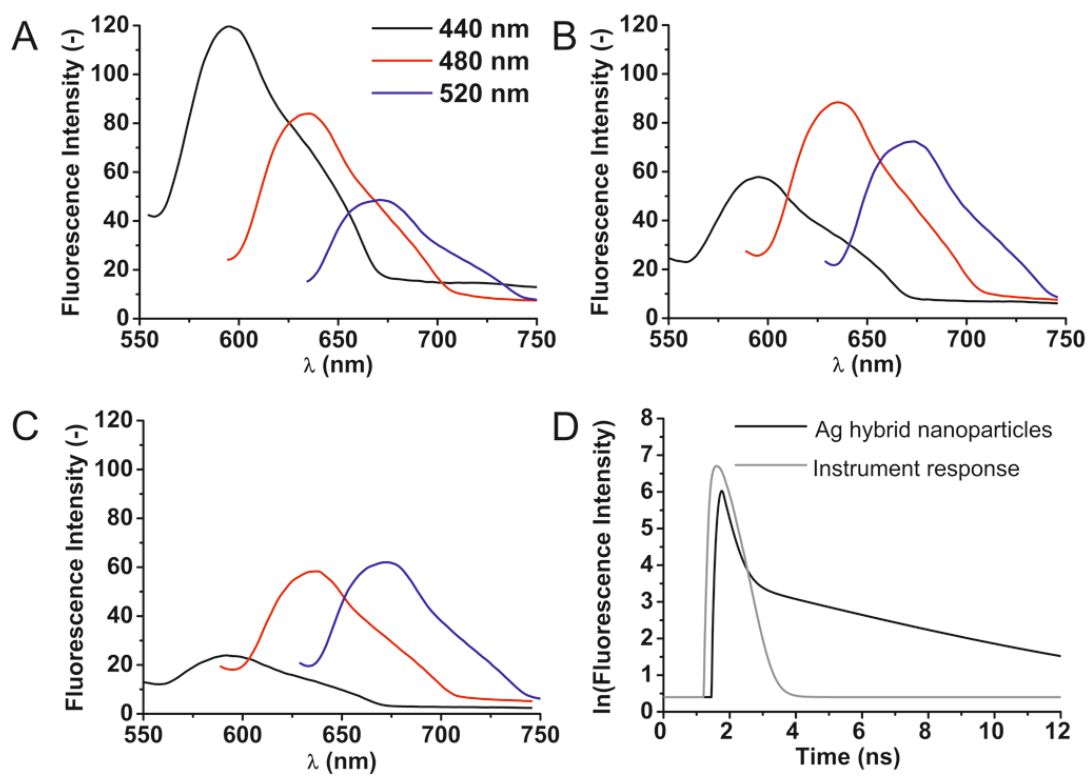

Figure 3.5. Fluorescence spectra of chemically reduced $\mathrm{AB}-\mathrm{DO} 3 \mathrm{~A}: \mathrm{AgNO}_{3}$ solutions (after $24 \mathrm{~h}$ ) at different excitation wavelengths, namely $\lambda_{\text {exc }} 440 \mathrm{~nm}, 480 \mathrm{~nm}$ and $520 \mathrm{~nm}$. A) 10:1 AB-DO3A: $\mathrm{AgNO}_{3}$ solution. B) 10:2 AB-DO3A:AgNO ${ }_{3}$ solution. C) 10:3 AB-DO3A:AgNO 3 solution. D) Fluorescence lifetime (black line) of the 10:2 $\mathrm{AB}-\mathrm{DO} 3 \mathrm{~A}: \mathrm{AgNO}_{3}$ solution (diluted) with excitation at $450 \mathrm{~nm}$. The instrument response (grey line) is also shown.

For all three solutions, shifting of the emission maxima to longer wavelengths upon increase of the excitation wavelength used is observed, which is often associated to a distribution of different NC sizes. ${ }^{28,32-34}$ Interestingly, the highest fluorescence intensity is observed in the 10:1 ABDO3A: $\mathrm{AgNO}_{3}$ sample (Figure 3.5A), which might be explained by the fact that, at this ratio, less AgNPs are formed and, therefore, more carboxylate groups are available for $\mathrm{Ag}_{n} \mathrm{NC}$ stabilization. ${ }^{34}$ This hypothesis is further supported by the UV absorption data, as a dramatic increase in the 
intensity at $400 \mathrm{~nm}$ (attributed to AgNPs) for the 10:2 and the 10:3 ABDO3A: $\mathrm{AgNO}_{3}$ samples is observed.

Other optical properties of the $\mathrm{Ag}_{n} \mathrm{NCs}$ formed under the previously described conditions were explored using lifetime measurements. In order to remove the AgNPs from the solution, a 10:2 AB-DO3A: $\mathrm{AgNO}_{3}$ sample was centrifuged at $11000 \mathrm{rpm}$ for 10 minutes. The supernatant (still containing $\mathrm{Ag}_{n} \mathrm{NCs}$ ) was subsequently diluted 10 times and the lifetime was measured. The decay of this sample could be fitted to a biexponential function with a slow component of $\tau_{1} 4.3 \mathrm{~ns}$ and a fast component of $\tau_{2}$ 245 ps (Figure 3.5D). Bifunctionally fitted decays with comparable values have previously been observed for multiple population $(n=2-5) A_{n} N C s$ grown in both peptides (1.9-2.9 ns and 73-420 ps) ${ }^{47}$ and polymer matrixes (2.4-3.1 ns and $960 \mathrm{ps}){ }^{17}$

A well-established technique used to determine the exact number of $\mathrm{Ag}$ atoms in NCs is mass spectrometry (MS). ${ }^{28,29,35,36,48}$ Chemical species containing $\mathrm{Ag}$ can be easily identified by the characteristic isotope pattern generated by the two abundant isotopes ${ }^{107} \mathrm{Ag}$ and ${ }^{109} \mathrm{Ag}$. Figure 3.6 shows the matrix-assisted laser desorption ionization (MALDI)-MS spectrum of three solutions of 10:1, 10:2 and 10:3 $\mathrm{AB}-\mathrm{DO} 3 \mathrm{~A}: \mathrm{AgNO}_{3}$ ratios, obtained in the negative ion mode. Only three sets of peaks centered at $\mathrm{m} / \mathrm{z} 670.8$, 894.9 and 1119 were successfully identified as Ag-containing compounds in all three samples (labeled 1, 2 and 3 in Figure 3.6, respectively). The isotope's unity spacing indicates that the corresponding species bear a -1 charge. The highest intensity for all three peaks was observed in the 10:2 sample (Figure 3.6A) and the isotope pattern agrees well with the presence of two $\mathrm{Ag}$ atoms embedded in AB-DO3A. The isotopic pattern and $\mathrm{m} / \mathrm{z}$ values of the set of peaks $1(\mathrm{~m} / \mathrm{z}=670.8$, theoretical molecular weight: 671.02), corresponding to the ion $\left[\mathrm{Ag}_{2} \mathrm{~L}-\mathrm{H}+\mathrm{K}\right]^{-}(\mathrm{L}=\mathrm{AB}-\mathrm{DO} 3 \mathrm{~A}$, Figure 3.6B), match the simulated isotopic pattern in Figure 3.6C. The same 
isotopic pattern is observed for peaks 2 and 3 , but their $\mathrm{m} / \mathrm{z}$ values (theoretical molecular weights: 895.09 and 1119.16 respectively) are higher due to the presence of one or two molecules of the matrix (sinapic acid, Figure 3.11 in Appendix 3.2).
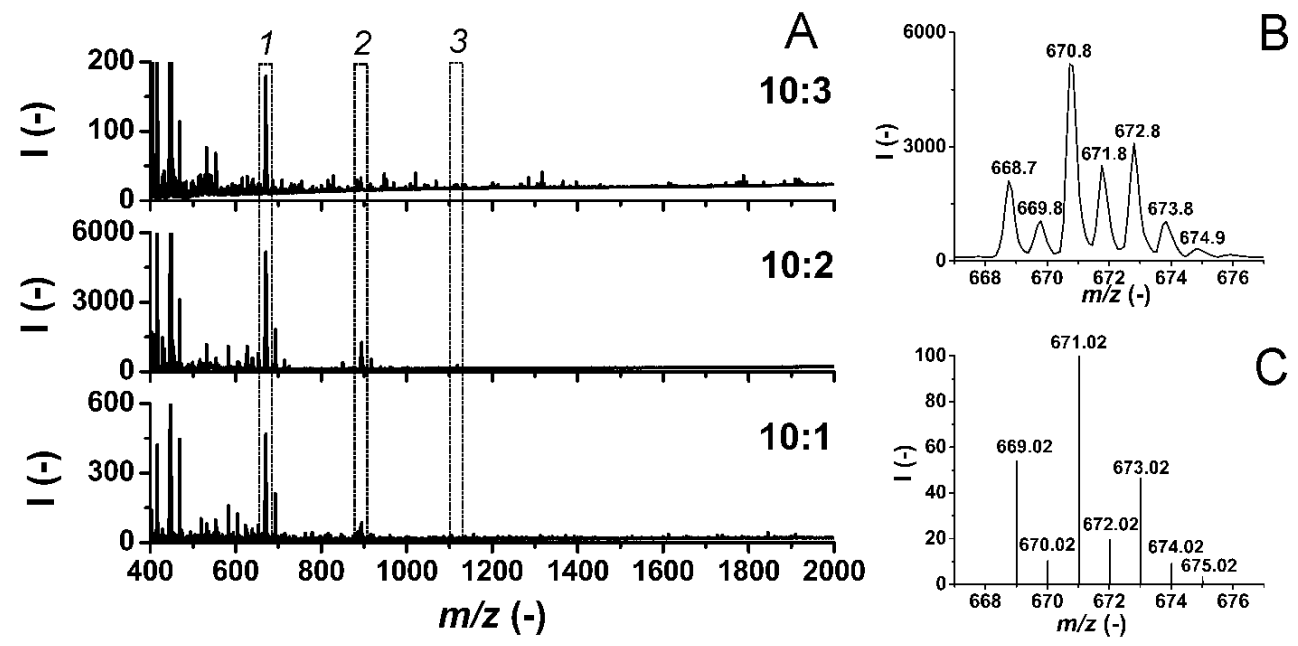

Figure 3.6. MALDI-MS spectra. A) Chemically reduced 10:1, 10:2 and 10:3 ABDO3A: $\mathrm{AgNO}_{3}$ solutions. Ag-containing species: 1) $\left[\mathrm{Ag}_{2} \mathrm{~L}-\mathrm{H}+\mathrm{K}\right]^{-}$; 2) $\left[\mathrm{Ag}_{2} \mathrm{~L}-\mathrm{H}+\mathrm{K}+\mathrm{M}\right]^{-}$; 3) $\left[\mathrm{Ag}_{2} \mathrm{~L}-\mathrm{H}+\mathrm{K}+2 \mathrm{M}\right]^{-}$; $\mathrm{L}$ is $\mathrm{AB}-\mathrm{DO} 3 \mathrm{~A}, \mathrm{M}$ is the matrix (sinapic acid). B) Amplification of the set of peaks $1(\mathrm{~m} / \mathrm{z}=670.8)$. C) Simulated isotopic pattern of 1 showing the characteristic isotopic pattern of $\mathrm{Ag}$.

In all cases, no assignable peaks for $\mathrm{Ag}_{1}$ or any other $\mathrm{Ag}$ containing species with a number of atoms higher than two could be identified. While this observation suggests that (within the scaffold) only $\mathrm{Ag}_{2} \mathrm{NCs}$ are formed, the steady-state and lifetime fluorescence data show that the presence of other $\mathrm{Ag}_{\mathrm{n}} \mathrm{NC}$ populations cannot be excluded.

\subsubsection{Interaction $A B-D O 3 A / A g$ Before and After Reduction}

To determine the initial metal-to-ligand complex formation and its possible stoichiometry, a titration of $\mathrm{AB}-\mathrm{DO} 3 \mathrm{~A}$ with $\mathrm{Ag}^{+}$was carried out and the $\overline{76}$ 
chemical shifts ( $\delta$ ) of the different protons of AB-DO3A were monitored by ${ }^{1} \mathrm{H}$-NMR. The differences between ${ }^{1} \mathrm{H}$-NMR spectra of the scaffold in the presence of $\mathrm{Ag}^{+}$at different concentrations are shown in Figure 3.7A-D. The peaks corresponding to $\mathrm{H}_{\mathrm{a}, \mathrm{a}, \mathrm{b}, \mathrm{b}, \mathrm{d}, \mathrm{e}, \mathrm{f}}$ shift as the concentration of $\mathrm{Ag}^{+}$ increases, therefore corroborating that indeed $\mathrm{Ag}^{+}$ions are captured by the acetate arms $\left(\mathrm{H}_{\mathrm{a}, \mathrm{a}^{\prime}, \mathrm{b}}\right)$ and that the $\mathrm{Ag}^{+}$ions are in close vicinity of the cyclen core $\left(H_{c, d, e, t}\right)$. Conversely, the chemical shifts of protons $H_{i}$ and $H_{j}$ do not change upon addition of $\mathrm{Ag}^{+}$, indicating that the aminobutyl arm does not participate in the initial complexation process to the metal ion precursor. Likewise, protons $H_{i}$ and $H_{j}$ remain unaltered after addition of $\mathrm{NaBH}_{4}$ (Figure 3.7E), thereby strongly suggesting that both the cyclen core and the carboxylic acids of the three acetate pendant arms (and not the aminobutyl arm) contribute to the stabilization of the AgNPs formed.

The chemical shifts corresponding to protons $\mathrm{H}_{\mathrm{a}, \mathrm{a}^{\prime}, \mathrm{b}, \mathrm{c}, \mathrm{d}, \mathrm{e}, \mathrm{f}}$ of $\mathrm{AB}-\mathrm{DO} 3 \mathrm{~A}$ upon titration of the precursor $\mathrm{Ag}^{+}$salt were fitted collectively to several complexation models with different binding stoichiometries (assuming independent binding sites, see Appendix 3.3). AB-DO3A can bind (at least) three $\mathrm{Ag}^{+}$atoms with an intrinsic equilibrium constant of $4 \times 10^{3} \mathrm{M}^{-1}$. The data and modeling do not imply any cooperativity or preference for the formation of a complex with a particular stoichiometry. Therefore, any preference for the formation of only one population of $\mathrm{Ag}_{n} \mathrm{NCs}$ (e.g., $\mathrm{Ag}_{2} \mathrm{NCs}$, as the MS data suggest) cannot be attributed to any predilection during the preceding $\mathrm{Ag}^{+}$complexation step, and hence must occur during or shortly after the reduction step. 


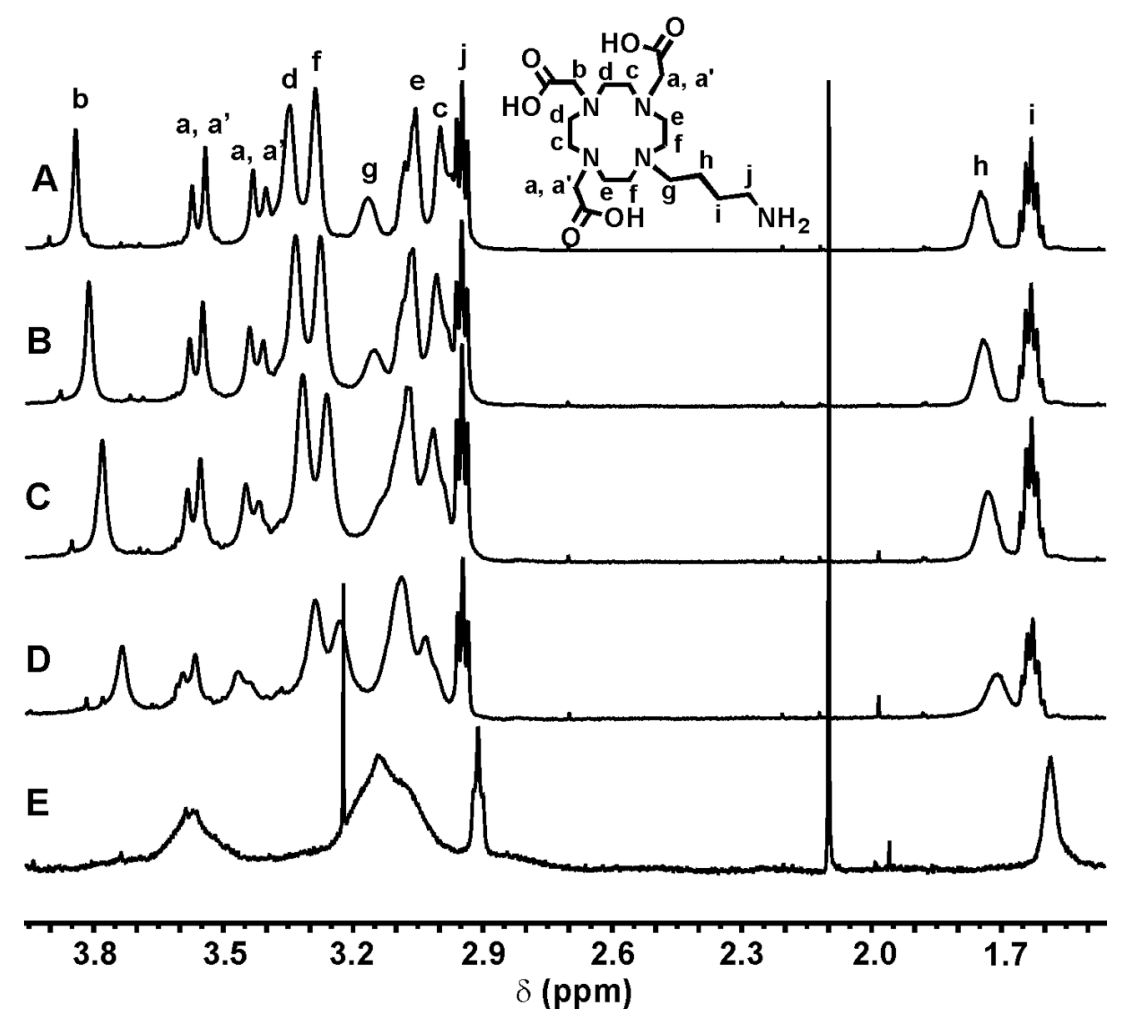

Figure 3.7. ${ }^{1} \mathrm{H}-\mathrm{NMR}$ spectra, including peak assignment and structure of $\mathrm{AB}-\mathrm{DO} 3 \mathrm{~A}$. A-D) Relevant spectra of $A B-D O 3 A$ in $\mathrm{D}_{2} \mathrm{O}$ upon addition of aliquots of $10 \mathrm{mM} \mathrm{AgNO}_{3}$ solution (see Appendix 3.3). $\mathrm{AgNO}_{3} / \mathrm{AB}-\mathrm{DO} 3 \mathrm{~A}$ concentrations $(\mathrm{mM} / \mathrm{mM})$ : A) 0/10; B) $2.65 / 7.35$; C) $4.60 / 5.40$ and D) $6.60 / 3.40$. E) Spectrum after chemical reduction $\left(\mathrm{AgNO}_{3} / \mathrm{AB}-\mathrm{DO} 3 \mathrm{~A}\right.$ ratio: 0.88$)$. Note that the highest $\mathrm{AB}-\mathrm{DO} 3 \mathrm{~A}: \mathrm{AgNO}_{3}$ ratio studied $(10: 3)$ is reached after the second titration point $(B)$.

The by NMR proposed role of the cyclen core and the carboxylic moieties with respect to the stabilization of the newly formed AgNPs was further reinforced by infrared spectroscopy (Figure 3.8). After reduction, a decrease in the intensity of the $\mathrm{C}=\mathrm{O}$ stretch frequency band corresponding to the carboxylic moieties of AB-DO3A (1665-1645 $\left.\mathrm{cm}^{-1}\right)$ is observed, as well as two new frequency bands that can be attributed to $\mathrm{N}-\mathrm{H}$ bending $\left(1595 \mathrm{~cm}^{-1}\right)$ and stretching $\left(3195 \mathrm{~cm}^{-1}\right)$ vibrations. From these observations 
it can be concluded that the aminobutyl arm of $A B-D O 3 A$ does not contribute to the stabilization of the AgNPs formed.

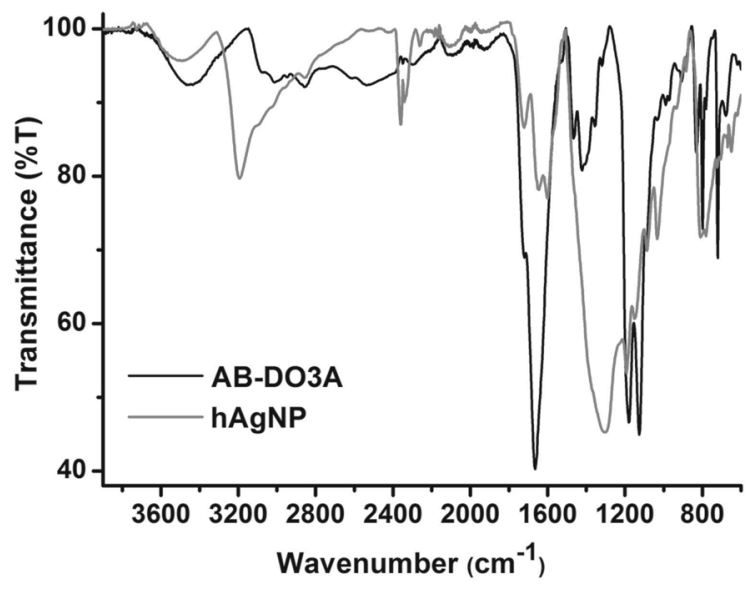

Figure 3.8. IR Spectra of AB-DO3A (black line) and hAgNSs (grey line).

\subsubsection{The Hybrid AgNP-AgnNC System}

The potential scaffold-mediated physical connection of the NCs to the NPs or hybrid nature of the AgNSs formed was tested by centrifuging a 10:2 $\mathrm{AB}-\mathrm{DO} 3 \mathrm{~A}: \mathrm{AgNO}_{3}$ solution after reduction and measuring the UV absorption and fluorescence emission at the maximum emission value $(635 \mathrm{~nm})$ before and after centrifugation. This was done by centrifuging the sample at $11000 \mathrm{rpm}$ for 10 minutes and (re)dissolving the pellet in the same water volume as the original solution. Assuming that any independently formed $\mathrm{Ag}_{n} \mathrm{NCs}$ (so not physically connected to AgNPs) do not precipitate out of solution after centrifugation, any fluorescence observed in the re-dissolved pellet must be related to species physically connected to the AgNPs. Figure 3.9A and B, which correspond to the absorption and fluorescence of the solution before centrifugation (black line) and of the pellet after centrifugation (red line), respectively, show that the ratio initial:final absorbance and initial:final fluorescence is, in both 
cases, the same $(\sim 2)$. This is clearly not the case when comparing the initial solution with the supernatant (Figure 3.9C and D, green lines), as the ratios initial:final absorbance and initial:final fluorescence are different (4 and 8 respectively). While still some NPs are observed in the supernatant after centrifugation, very weak fluorescence is detected. Since all of our previous observations corroborate the fact that both $\mathrm{Ag}_{\mathrm{n}} \mathrm{NCs}$ as well as AgNPs are present in solution, this last observation strongly suggests some degree of attachment between these two species. Hence, we foresee a physical connection between the NPs and the NCs, thereby confirming the formation of $h A g N S s$. Lastly, while a feasible role of the ABDO3A in the AgNP stabilization is clearly portrayed in Figure 3.1, how this scaffold stabilizes the $\mathrm{Ag}_{n} \mathrm{NCs}$ formed is not fully understood yet.
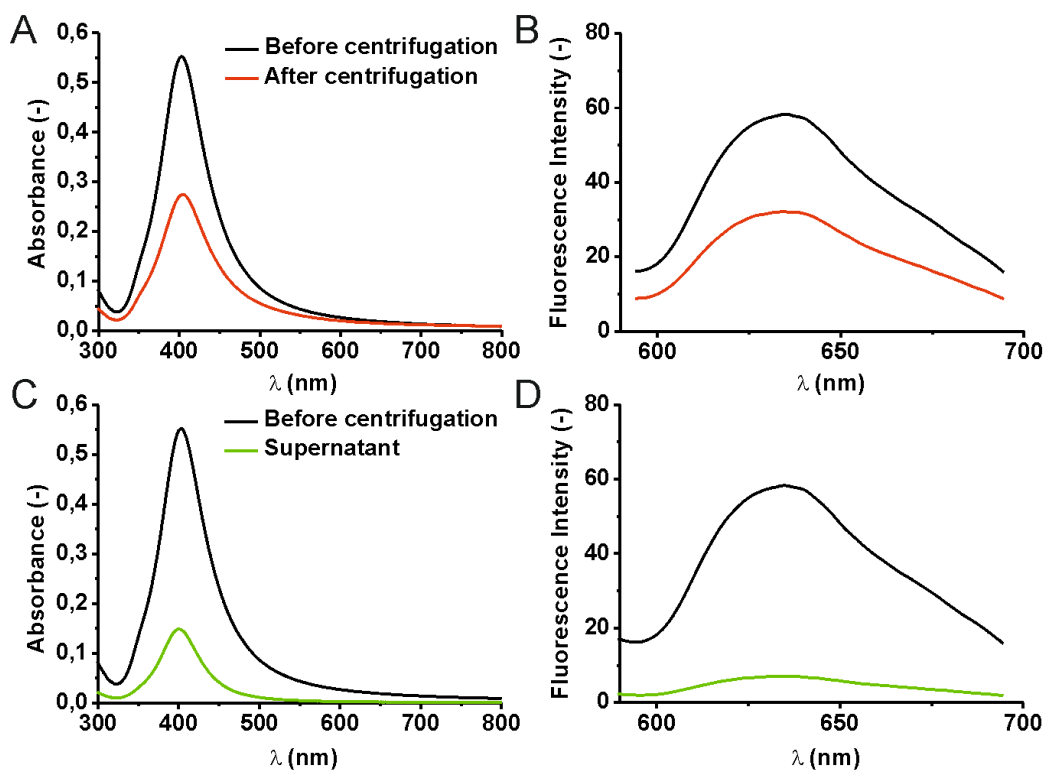

Figure 3.9. Spectra of chemically-reduced 10:2 AB-DO3A: $\mathrm{AgNO}_{3}$ solution. The black line corresponds to the spectrum before centrifugation, the red line to the redispersed pellet (the same initial water volume) and the green line to the supernatant. A, C) UV spectra. $B, D)$ Fluorescence emission $\left(\lambda_{\text {exc }} 480 \mathrm{~nm}\right.$ ) spectra. 
Overall, AB-DO3A-like scaffolds are believed to be an interesting platform for engineering fluorescent $h$ AgNSs with precise size of both NPs and NCs. This control is thought to arise from the lower amount of degrees of freedom compared to bigger organic scaffolds, where the growth of clusters is governed by a large number of parameters such as $\mathrm{pH}$, shape and conformation in space. ${ }^{30,31,33}$ Nevertheless, proper characterization of such systems proved to be challenging as fairly established techniques such as MS did not seem to provide full information with respect to the $\mathrm{Ag}_{n} \mathrm{NC}$ composition.

\subsection{Conclusions}

Altogether, the chemical reduction of $\mathrm{AgNO}_{3}$ in the presence of the $\mathrm{AB}$ DO3A scaffold yields AgNPs stabilized by the AB-DO3A scaffold that, at the same time, stabilizes fluorescent $\mathrm{Ag}_{\mathrm{n}} \mathrm{NCs}$. While $\mathrm{MS}$ is a fairly established, standard technique for the quantification of the number atoms in $\mathrm{Ag}_{n} \mathrm{NCs},{ }^{28,29,35,36,48}$ our steady-state fluorescence studies reveal the presence of other $\mathrm{Ag}_{\mathrm{n}} \mathrm{NC}$ populations not detected by MS. Therefore, the formation of NCs of various sizes, grown in or outside of the scaffold, cannot be ruled out. While fluorescent AgNPs have previously been described in the literature ${ }^{49,50}$ this is, to our knowledge, the first example of multifunctional $h$ AgNSs grown using a polyamino carboxylic acid scaffold. This new class of hybrid nanostructures opens up the door for a simpler, straightforward controlled synthesis of multifunctional materials for molecular imaging and (possibly) therapeutic purposes. 


\subsection{Experimental}

Materials. Commercial grade reagents were purchased from Sigma Aldrich and $\mathrm{TCl}$ Europe and were used without further purification unless otherwise stated. N-(4-bromobutyl)phthalimide was obtained from Sigma Aldrich. Solvents were dried over molecular sieves. Deuterated solvents were purchased from Cambridge Isotope Laboratories and NMR tubes from Wilmad LabGlass. All Ultrapure (Milli-Q) water was used in all experiments.

\section{Synthesis of 1,4,7,10-tetraaza-1-(4-butylphthalimide)-4,7,10-} tris (tert-butoxycarboxymethyl) cyclododecane

(Bphth-

tbutylD03A, 3). In a three-neck round-bottom flask, $\mathrm{K}_{2} \mathrm{CO}_{3}(420 \mathrm{mg}$, $3.04 \mathrm{mmol}$ ) was added to a stirring solution of 1,4,7-Tris(tertbutoxycarboxymethyl)-1,4,7,10-tetraazacyclododecane 1 (DO3A, synthesized as previously described in literature $)^{51}(500 \mathrm{mg}, 0.97 \mathrm{mmol})$ in $40 \mathrm{~mL}$ dry acetonitrile. The mixture was kept under $\mathrm{N}_{2}$ for 10 minutes under vigorous stirring. Afterwards, N-(4-bromobutyl)phthalimide (2) (274 mg, $0.97 \mathrm{mmol}$ ) dissolved in $10 \mathrm{~mL}$ dry acetonitrile was added slowly to the reaction mixture and allowed to react for 2 days at $50^{\circ} \mathrm{C}$. The mixture was left to cool, filtered to remove inorganic salts and the solvent evaporated under reduced pressure. The desired compound was obtained as a yellow oil after chromatography on alumina $\left(94: 6 \mathrm{CH}_{2} \mathrm{Cl}_{2}: \mathrm{MeOH}, \mathrm{R}_{\mathrm{f}}\right.$ 0.5). Yield: $70 \%$ (489 mg, $0.683 \mathrm{mmol}) .{ }^{1} \mathrm{H} \mathrm{NMR}\left(\mathrm{CDCl}_{3}, 600 \mathrm{MHz}\right) \delta(\mathrm{ppm})$ : $7.86(\mathrm{~m}, 2 \mathrm{H}), 7.75(\mathrm{~m}, 2 \mathrm{H}), 3.66(\mathrm{t}, J(\mathrm{H}, \mathrm{H})=7.3 \mathrm{~Hz}, 2 \mathrm{H}), 3.6-2.1(\mathrm{bs}, 24 \mathrm{H})$, $1.63(\mathrm{~m}, J(\mathrm{H}, \mathrm{H})=7.3,7.6 \mathrm{~Hz}, 2 \mathrm{H}), 1.45$ (bs, 2H), 1.45-1.49 (m, 27H). ${ }^{13} \mathrm{C}$ $\mathrm{NMR}\left(\mathrm{CDCl}_{3}, 150 \mathrm{MHz}\right) \delta$ (ppm): 172.7, 168.3, 134.1, 132.1, 123.2, 82.7, 
82.2, 56.5, 55.8, 53.8, 52.8, 50, 47.95, 37.6, 28, 27.8, 26.8, 23.7. MS (ESI+): $m / z: 738.3\left[\mathrm{M}^{+}+\mathrm{Na}\right]$.

\section{Synthesis of 1,4,7,10-tetraaza-1-(4-aminobutyl)-4,7,10-tris (tert-} butoxycarboxymethyl)cyclododecane (AB-tbutylD03A, 4). Compound 4 was synthesized using a procedure adapted from literature. ${ }^{52}$ To a stirring solution of 3 (450 $\mathrm{mg}, 0.63 \mathrm{mmol})$ in ethanol, $100 \%$ hydrazine monohydrate $(1.4 \mathrm{~mL}, 28.35 \mathrm{mmol})$ was added and the solution was allowed to react at $50^{\circ} \mathrm{C}$ for 2 days. The mixture was evaporated under reduced pressure and the residue was co-evaporated with ethanol three times in order to remove excess hydrazine. Afterwards, $40 \mathrm{~mL}$ of $\mathrm{CHCl}_{3}$ were added to the residue and the mixture was filtered after sonication. The product was extracted from the organic phase acid water (acetic acid, $\mathrm{pH} 3,3 \times 20 \mathrm{~mL}$ ). Subsequently, the $\mathrm{pH}$ of the combined aqueous phases was adjusted to 10 with $1 \mathrm{M} \mathrm{NaOH}$ and the final product extracted from the aqueous phase with chloroform $(3 \times 30 \mathrm{~mL})$. After drying the combined organic phases over $\mathrm{MgSO}_{4}$, the solvent was evaporated under reduced pressure and the desired compound was obtained as a yellow oil. Yield: $53 \%$ (197 mg, $0.34 \mathrm{mmol}$ ). ${ }^{1} \mathrm{H}$ NMR ( $\left.\mathrm{CDCl}_{3}, 600 \mathrm{MHz}\right) \delta$ (ppm): 3.31-3.29 (s, 6H), 2.86-2.48 (bs, 20H) $1.89(\mathrm{~m}, 2 \mathrm{H}), 1.76(\mathrm{~m}, 2 \mathrm{H}), 1.46-1.48(\mathrm{~m}, 27 \mathrm{H})$. ${ }^{13} \mathrm{C}$ NMR $\left(\mathrm{CDCl}_{3}, 150 \mathrm{MHz}\right) \delta(\mathrm{ppm}): 171.8,171.6,81.9,81.4,57.2,56.9$, 54.3, 50.9, 39, 28.1, 28.07, 26.8, 25.8. MS (ESI+): $\mathrm{m} / \mathrm{z}: 586.0\left[\mathrm{M}^{+}+\mathrm{H}\right]$.

\section{Synthesis of 1,4,7,10-tetraaza-1-(4-aminobutyl)-4,7,10-triacetic} acid cyclododecane (AB-D03A, 5). In a round-bottom flask, TFA ( $5 \mathrm{~mL}, 67 \mathrm{mmol})$ was added to a solution of 4 (200 mg, $0.34 \mathrm{mmol})$ in $5 \mathrm{~mL}$ $\mathrm{CH}_{2} \mathrm{Cl}_{2}$ and the reaction mixture was allowed to stir at room temperature overnight. The mixture was evaporated under reduced pressure and the 
residue co-evaporated with dichloromethane three times in order to remove excess TFA. Subsequently, the residue was re-dissolved in water and washed with dichloromethane three times. The aqueous solution was freeze dried and subsequently triturated with diisopropyl ether. Compound 5 was obtained as a yellow oil (quantitative yield). ${ }^{1} \mathrm{H}$ NMR $\left(\mathrm{D}_{2} \mathrm{O}, 600 \mathrm{MHz}\right)$

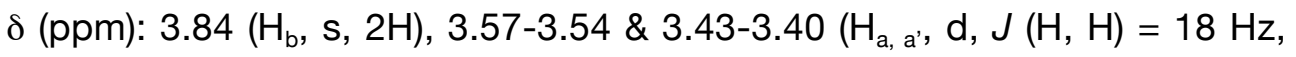
$4 \mathrm{H}), 3.35\left(\mathrm{H}_{\mathrm{d}}\right.$, bs, $\left.4 \mathrm{H}\right), 3.29\left(\mathrm{H}_{\mathrm{f}}, \mathrm{bs}, 4 \mathrm{H}\right), 3.16\left(\mathrm{H}_{\mathrm{g}}\right.$, bs, $\left.2 \mathrm{H}\right), 3.06\left(\mathrm{H}_{\mathrm{e}}, \mathrm{bs}, 4 \mathrm{H}\right)$, $3\left(\mathrm{H}_{\mathrm{c}}\right.$, bs, 4H), $2.95\left(\mathrm{H}_{\mathrm{j}}, \mathrm{t}, J(\mathrm{H}, \mathrm{H})=7.6 \mathrm{~Hz}, 2 \mathrm{H}\right), 1.75\left(\mathrm{H}_{\mathrm{h}}, \mathrm{bs}, 2 \mathrm{H}\right), 1.63$ $\left.\left(\mathrm{H}_{\mathrm{i}}, \mathrm{m}, J(\mathrm{H}, \mathrm{H})=7.6,7.8 \mathrm{~Hz}, 2 \mathrm{H}\right) \cdot{ }^{13} \mathrm{C} \mathrm{NMR}\left(\mathrm{D}_{2} \mathrm{O}\right), 150 \mathrm{MHz}\right) \delta(\mathrm{ppm}): 174.1$, 173.9, 169.4, 55.3, 53.3, 53, 52.8, 51.3, 49.8, 48.4, 48, 38.5, 23.6, 20.1. MS (ESI+): $m / z: 418.3\left[\mathrm{M}^{+}+\mathrm{H}\right]$.

UV-Vis Spectroscopy. UV spectra were acquired on a Perkin Elmer Lambda 850 UV-Vis spectrometer. All samples were measured in UV quality quartz cuvettes with a path length of $1 \mathrm{~cm}$.

Transmission Electron Microscopy (TFM). TEM images were obtained using analytical TEM FEI instruments. In order to prepare the samples, $5 \mu \mathrm{L}$ of the desired sample was applied onto Formvar-carbon coated grids (from electron microscopy sciences). After leaving the sample for 1 minute, the excess of liquid was removed using a piece of filter paper. The size distribution of hAgNPs was determined by measuring the particle core diameter of more than 80 counts from TEM images using Image $\mathrm{J}$ software. Energy-dispersive $\mathrm{X}$-ray analysis was done using a Thermo Noran System 6 with a resolution of $138 \mathrm{eV}$ on a Manganese standard. 
Dynamic Light Scattering (DLS). The hydrodynamic sizes of individual $h$ AgNPs were characterized by dynamic light scattering using a Zetatrac Microtrac Flex 10.6.1 particle analyzer with a $180^{\circ}$ back-scatter (Microtrac Inc., PA, USA). The particle diameter was calculated using a heterodyne technique to obtain a characteristic frequency, which is then related to the particle diameter by the Stokes-Einstein equation. $1 \mathrm{~mL}$ hAgNPs samples were inserted in a cell inside the DLS-setup. The size distribution of the nanoparticles was determined by measuring their diameter for more than 450 counts. The number-average diameter was recorded for the sample and averaged over 5 measurements.

\section{Nuclear Magnetic Resonance (NMR) Characterization and}

Titration. All NMR experiments were performed on a Bruker Avance II NMR spectrometer operating at $600.35 \mathrm{MHz}$ for ${ }^{1} \mathrm{H}$ and $150.09 \mathrm{MHz}$ for ${ }^{13} \mathrm{C}$. Chemicals shifts are given in ppm using the residual solvent signal as reference. The multiplicity of the peaks is reported using the following abbreviations: $\mathrm{s}=$ singlet, $\mathrm{d}=$ doublet, $\mathrm{t}=$ triplet, quint $=$ quintuplet, $\mathrm{m}=$ multiplet. All NMR spectra were processed using TOPSPIN 2.1 and MestReC software packages.

Mass Spectrometry. Mass spectra were acquired on a Waters Micromass LCT (ESI) mass spectrometer and on a applied Biosystems Voyager-DE RP (MALDI-TOF) mass spectrometer, using sinapic acid as matrix. ${ }^{36}$

Fluorescence Spectroscopy. All steady-state spectra were recorded using a Perkin Elmer Fluorescence Spectrometer. 
Infrared Spectroscopy. All IR spectra were recorded using a Fourier Transform (FT-IR) Thermo Scientific Spectrometer.

Lifetime Measurements. Lifetime measurements were done using a single-molecule confocal microscope consisting of an Olympus IX71 microscope equipped with a PicoQuant Diode Laser, an Acousto Optic tunable filter from Crystal Technologies, a single mode fiber, objectives from Olympus (60x/1.20 water) and Nikon (Plan APO) and an ADP with an active area diameter of $100 \mu \mathrm{m}$. Excitation was done at $450 \mathrm{~nm}$ and a 488 $\mathrm{nm}$ long-pass filter was used during measurements.

\subsection{References}

(1) Günes, S.; Sariciftci, N. S. Inorg. Chim. Acta 2008, 361, 581.

(2) Saunders, B. R.; Turner, M. L. Adv. Colloid Interface Sci. 2008, 138, 1.

(3) García-Barrasa, J.; López-de-Luzuriaga, J.; Monge, M. Cent. Eur. J. Chem. 2011, 9, 7 .

(4) Bhattacharya, R.; Mukherjee, P. Adv. Drug Delivery Rev. 2008, 60, 1289.

(5) Bhattacharyya, S.; Kudgus, R.; Bhattacharya, R.; Mukherjee, P. Pharm. Res. 2011, 28, 237.

(6) Thanh, N. T. K.; Green, L. A. W. Nano Today 2010, 5, 213.

(7) Banin, U.; Millo, O. In Nanoparticles: from Theory to Application; Schmid, G., Ed.; Wiley-VCH Verlag GmbH \& Co. KGaA: Weinheim, FRG, 2005, p 305.

(8) Bradley, J. S.; Schmid, G.; Talapin, D. V.; Shevchenko, E. V.; Weller, H. In Nanoparticles: from Theory to Application; Schmid, G., Ed.; Wiley-VCH Verlag GmbH \& Co. KGaA: Weinheim, FRG, 2005, p 185.

(9) Jain, P. K.; Huang, X.; El-Sayed, I. H.; El-Sayed, M. A. Acc. Chem. Res. 2008, 41, 1578.

(10) Galdiero, S.; Falanga, A.; Vitiello, M.; Cantisani, M.; Marra, V.; Galdiero, M. Molecules 2011, 16, 8894.

(11) Nair, L. S.; Laurencin, C. T. J. Biomed. Nanotechnol. 2007, 3, 301.

(12) Premkumar, T.; Lee, Y.; Geckeler, K. E. Chem. Eur. J. 2010, 16, 11563.

(13) Wu, Q.; Cao, H.; Luan, Q.; Zhang, J.; Wang, Z.; Warner, J. H.; A. R. Watt, A. Inorg. Chem. 2008, 47, 5882.

(14) Wilcoxon, J. P.; Abrams, B. L. Chem. Soc. Rev. 2006, 35, 1162.

(15) Zheng, J.; Nicovich, P. R.; Dickson, R. M. Annu. Rev. Phys. Chem. 2007, $58,409$.

(16) Diez, I.; Ras, R. H. A. Nanoscale 2011, 3, 1963. 
(17) Díez, I.; Pusa, M.; Kulmala, S.; Jiang, H.; Walther, A.; Goldmann, A. S.; Müller, A. H. E.; Ikkala, O.; Ras, R. H. A. Angew. Chem., Int. Ed. 2009, 48, 2122.

(18) Patel, S. A.; Richards, C. I.; Hsiang, J.-C.; Dickson, R. M. J. Am. Chem. Soc. 2008, 130, 11602.

(19) Graf, P.; Mantion, A.; Foelske, A.; Shkilnyy, A.; Mašić, A.; Thünemann, A. F.; Taubert, A. Chem. Eur. J. 2009, 15, 5831.

(20) Abou-Okeil, A.; Amr, A.; Abdel-Mohdy, F. A. Carbohydr. Polym. 2012, 89, 1.

(21) Sun, Y.; Xia, Y. Science 2002, 298, 2176.

(22) Huang, H. H.; Ni, X. P.; Loy, G. L.; Chew, C. H.; Tan, K. L.; Loh, F. C.; Deng, J. F.; Xu, G. Q. Langmuir 1996, 12, 909.

(23) Tan, Y.; Dai, X.; Li, Y.; Zhu, D. J. Mater. Chem. 2003, 13, 1069.

(24) He, R.; Qian, X.; Yin, J.; Zhu, Z. J. Mater. Chem. 2002, 12, 3783.

(25) Sun, X.; Dong, S.; Wang, E. Macromolecules 2004, 37, 7105.

(26) Lesniak, W.; Bielinska, A. U.; Sun, K.; Janczak, K. W.; Shi, X.; Baker, J. R.; Balogh, L. P. Nano Lett. 2005, 5, 2123.

(27) Zheng, J.; Dickson, R. M. J. Am. Chem. Soc. 2002, 124, 13982.

(28) Petty, J. T.; Zheng, J.; Hud, N. V.; Dickson, R. M. J. Am. Chem. Soc. 2004, 126, 5207.

(29) Ritchie, C. M.; Johnsen, K. R.; Kiser, J. R.; Antoku, Y.; Dickson, R. M.; Petty, J. T. J. Phys. Chem. C 2006, 111, 175.

(30) Richards, C. I.; Choi, S.; Hsiang, J.-C.; Antoku, Y.; Vosch, T.; Bongiorno, A.; Tzeng, Y.-L.; Dickson, R. M. J. Am. Chem. Soc. 2008, 130, 5038.

(31) Zhang, J.; Xu, S.; Kumacheva, E. Adv. Mater. 2005, 17, 2336.

(32) Shen, Z.; Duan, H.; Frey, H. Adv. Mater. 2007, 19, 349.

(33) Shang, L.; Dong, S. Chem. Commun. 2008, 1088.

(34) Xu, H.; Suslick, K. S. ACS Nano 2010, 4, 3209.

(35) Udaya Bhaskara Rao, T.; Pradeep, T. Angew. Chem., Int. Ed. 2010, 49, 3925.

(36) Wu, Z.; Lanni, E.; Chen, W.; Bier, M. E.; Ly, D.; Jin, R. J. Am. Chem. Soc. 2009, 131, 16672.

(37) Cathcart, N.; Kitaev, V. J. Phys. Chem. C 2010, 114, 16010.

(38) Adhikari, B.; Banerjee, A. Chem. Mater. 2010, 22, 4364.

(39) Evanoff, D. D.; Chumanov, G. ChemPhysChem 2005, 6, 1221.

(40) Evanoff, D. D.; Chumanov, G. J. Phys. Chem. B 2004, 108, 13957.

(41) Kuo, P.-L.; Chen, W.-F. J. Phys. Chem. B 2003, 107, 11267.

(42) Manna, A.; Imae, T.; lida, M.; Hisamatsu, N. Langmuir 2001, 17, 6000.

(43) Schultz, D.; Gwinn, E. Chem. Commun. 2011, 47, 4715.

(44) Peyser, L. A.; Vinson, A. E.; Bartko, A. P.; Dickson, R. M. Science 2001, 291, 103.

(45) Xu, H.; Suslick, K. S. Adv. Mater. 2010, 22, 1078.

(46) Yu, J.; Choi, S.; Richards, C. I.; Antoku, Y.; Dickson, R. M. Photochem. Photobiol. 2008, 84, 1435.

(47) Yu, J.; Patel, S. A.; Dickson, R. M. Angew. Chem., Int. Ed. 2007, 46, 2028.

(48) Gwinn, E. G.; O'Neill, P.; Guerrero, A. J.; Bouwmeester, D.; Fygenson, D. K. Adv. Mater. 2008, 20, 279. 
(49) Maretti, L.; Billone, P. S.; Liu, Y.; Scaiano, J. C. J. Am. Chem. Soc. 2009, $131,13972$.

(50) Stamplecoskie, K. G.; Scaiano, J. C. J. Am. Chem. Soc. 2011, 133, 3913.

(51) Beeby, A.; Bushby, L. M.; Maffeo, D.; Gareth Williams, J. A. J. Chem. Soc., Dalton Trans. 2002, 48.

(52) Sasaki, T.; Minamoto, K.; Itoh, H. J. Org. Chem. 1978, 43, 2320. 


\section{Appendix 3.1. Control Fxperiments: Fluorescence of $\mathrm{AgNO}_{3}$ and AB-DO3A Solutions}
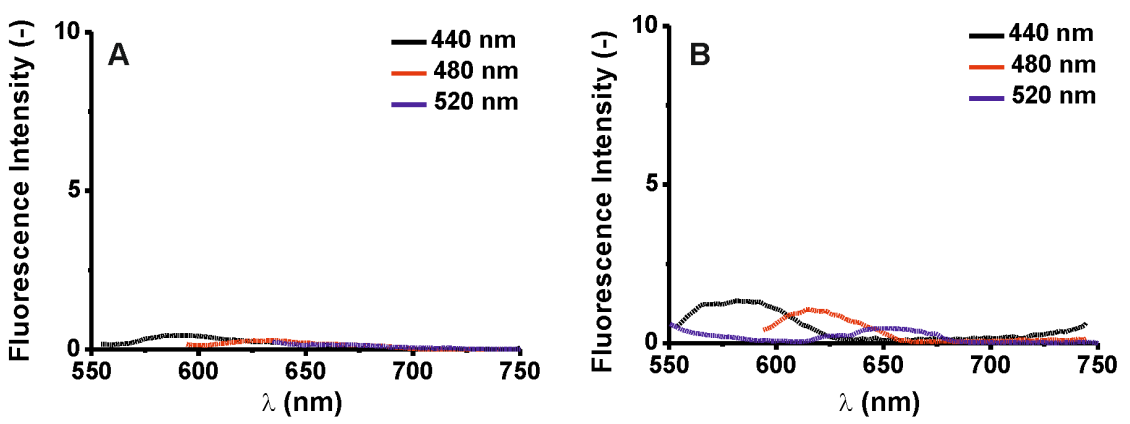

Figure 3.10. A) Fluorescence spectra (taken after $24 \mathrm{~h}$ ) of a $0.2 \mathrm{mM} \mathrm{AgNO}_{3}$ solution excited at $\lambda_{\text {excitation }}$ : 440, 480 and $520 \mathrm{~nm}$ (black, red and blue, respectively). B) Fluorescence spectra (taken after $24 \mathrm{~h}$ ) of a $1 \mathrm{mM}$ AB-DO3A solution excited at $\lambda_{\text {excitation }}: 440,480$ and $520 \mathrm{~nm}$ (black, red and blue, respectively). Note the very small intensity scale. 


\section{Appendix 3.2. Amplification of MALDI-MS Regions}

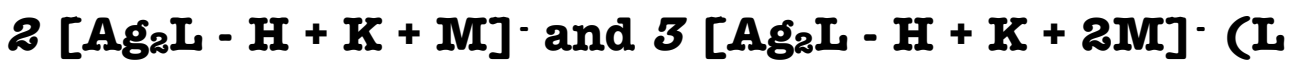

is AB-DO3A, $M$ is the matrix -sinapic acid-)
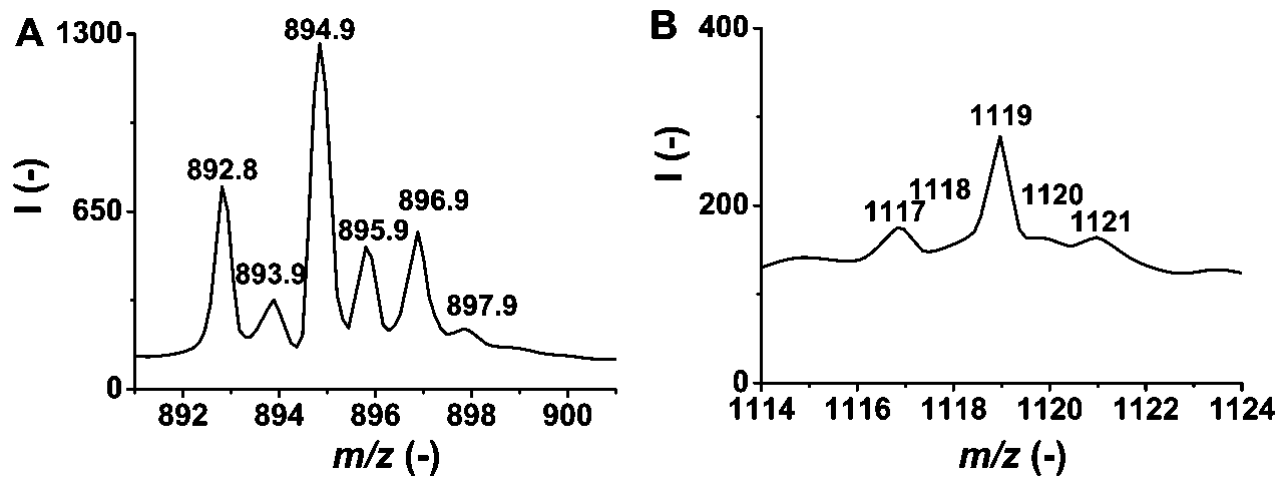

Figure 3.11. Amplification of MALDI-MS (-) regions: A) 2, B) 3. 


\section{Appendix 3.3. Titration of AB-D03A with $\mathbf{A g}^{+}$ Followed by ${ }^{1}$ H-NMR}

In order to study the complexation process, a $10 \mathrm{mM}$ solution of AB-DO3A in $\mathrm{D}_{2} \mathrm{O}$ was titrated with $\mathrm{Ag}^{+}$. This was done by adding $100 \mu \mathrm{L}$ of $\mathrm{AgNO}_{3}$ (10 $\mathrm{mM}$ in $\mathrm{D}_{2} \mathrm{O}$ ) to $600 \mu \mathrm{L}$ of $\mathrm{AB}-\mathrm{DO} 3 \mathrm{~A}$ in an NMR tube and measuring the spectrum. For each subsequent aliquot addition of $100 \mu \mathrm{L} \mathrm{AgNO}, 600 \mu \mathrm{L}$ of the previously measured solution were used and the NMR spectrum was taken. This procedure was repeated 15 times; although for the final fitting only 12 points were used due to overlapping of ${ }^{1} \mathrm{H}$ NMR signals. The

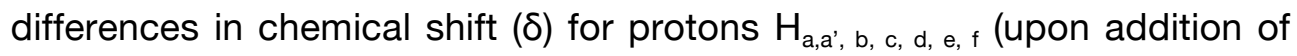
$\mathrm{Ag}^{+}$) were quantified and the equilibrium constants were calculated by non-linear least squares fitting all peaks simultaneously to a 1:2 and a 1:3 model, given by:

1:2 Model:

$\mathrm{L}+\mathrm{M} \stackrel{\mathrm{K}_{1}}{\longrightarrow} \mathrm{LM}+\mathrm{M} \stackrel{\mathrm{K}_{2}}{\longrightarrow} \mathrm{LM}_{2}$

1:3 Model:

$\mathrm{L}+\mathrm{M} \stackrel{\mathrm{K}_{1}}{\longrightarrow} \mathrm{LM}+\mathrm{M} \stackrel{\mathrm{K}_{2}}{\longrightarrow} \mathrm{LM}_{2}+\mathrm{M} \stackrel{\mathrm{K}_{3}}{\longrightarrow} \mathrm{LM}_{3}$

Where $L$ is $A B-D O 3 A$ and $M$ is $\mathrm{Ag}^{+}$and the equilibrium constants are given by:

$\mathrm{K}_{1}=\frac{[\mathrm{LM}]}{[\mathrm{L}][\mathrm{M}]} \quad \mathrm{K}_{2}=\frac{\left[\mathrm{LM}_{2}\right]}{[\mathrm{LM}][\mathrm{M}]} \quad \mathrm{K}_{3}=\frac{\left[\mathrm{LM}_{3}\right]}{\left[\mathrm{LM}_{2}\right][\mathrm{M}]}$ 


\section{1:2 Model}

The data was fitted to a 1:2 model assuming independent binding sites (Figure 3.12), where the calculated intrinsic equilibrium constant $\mathrm{K}_{\mathrm{i}}$ $\left(2.4 \times 10^{3} \mathrm{M}^{-1}\right)$ is given by:

$\mathrm{K}_{1} / 2=\mathrm{K}_{\mathrm{i}}=2 \mathrm{~K}_{2}$
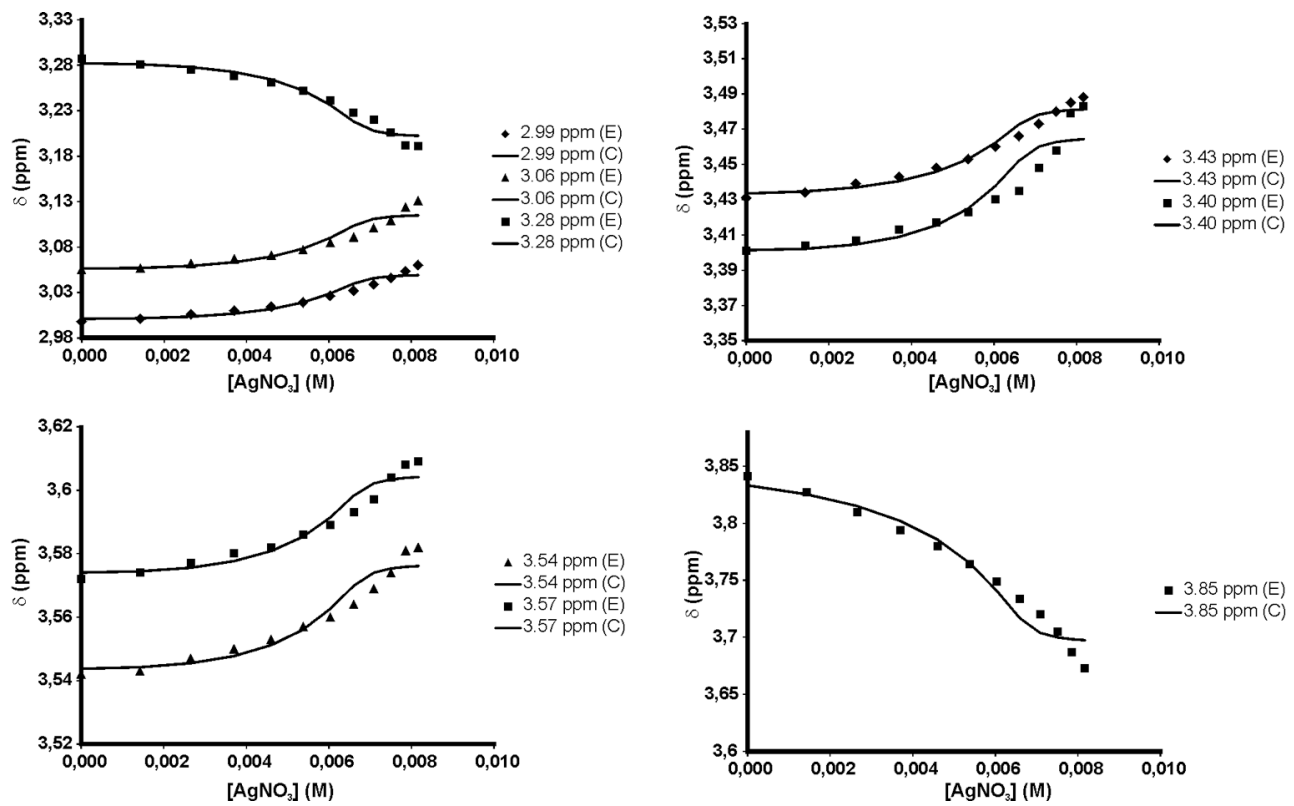

Figure 3.12. Titration curves, including experimental $(E)$ and calculated $(C)$ values, nonlinear least squares fitted to a 1:2 model assuming independent binding sites $\left(K_{i}=2.4 \times 10^{3} \mathrm{M}^{-1}\right)$, for the peaks $\mathrm{H}_{\mathrm{a}, \mathrm{a}^{\prime}, \mathrm{b}, \mathrm{c}, \mathrm{d}, \mathrm{e}, \mathrm{f}}$ of $\mathrm{AB}-\mathrm{DO} 3 \mathrm{~A}\left([\mathrm{AB}-\mathrm{DO} 3 \mathrm{~A}]_{\text {initial }}=10 \mathrm{mM}\right.$, $\left.\left[\mathrm{AgNO}_{3}\right]_{\mathrm{added}}=10 \mathrm{mM}, 25^{\circ} \mathrm{C}\right)$

The poor fitting of the data to the 1:2 model (Figure 3.12) demonstrates that a 1:2 complex is not the only species formed. Subsequently, the fitting of this data to less restrictive 1:2 models was checked, assuming positive cooperativity $\left(\mathrm{K}_{2}>\mathrm{K}_{1} / 4\right)$ as well as negative cooperativity $\left(\mathrm{K}_{2}<\mathrm{K}_{1} / 4\right)$. 
Assuming negative cooperativity $\left(\mathrm{K}_{1}=1 \times 10^{4} \mathrm{M}^{-1}\right.$ and $\left.\mathrm{K}_{2}=1.3 \times 10^{3} \mathrm{M}^{-1}\right)$, the quality of the fit did not change when compared to the $1: 2$ (independent binding sites) model, while for positive cooperativity $\left(\mathrm{K}_{1}=7 \times 10^{0} \mathrm{M}^{-1}\right.$ and $\left.\mathrm{K}_{2}=4 \times 10^{4} \mathrm{M}^{-1}\right)$ a slightly better fit was obtained.

\section{1:3 Model}

Given the poor fitting of the data to the $1: 2$ model, non-linear least squares fitting to a 1:3 model assuming independent binding sites (Figure 3.13) was performed, where the intrinsic equilibrium constant $\mathrm{K}_{\mathrm{i}}$ is given by:

$\mathrm{K}_{1} / 3=\mathrm{K}_{\mathrm{i}}=\mathrm{K}_{2}=3 \mathrm{~K}_{3}$
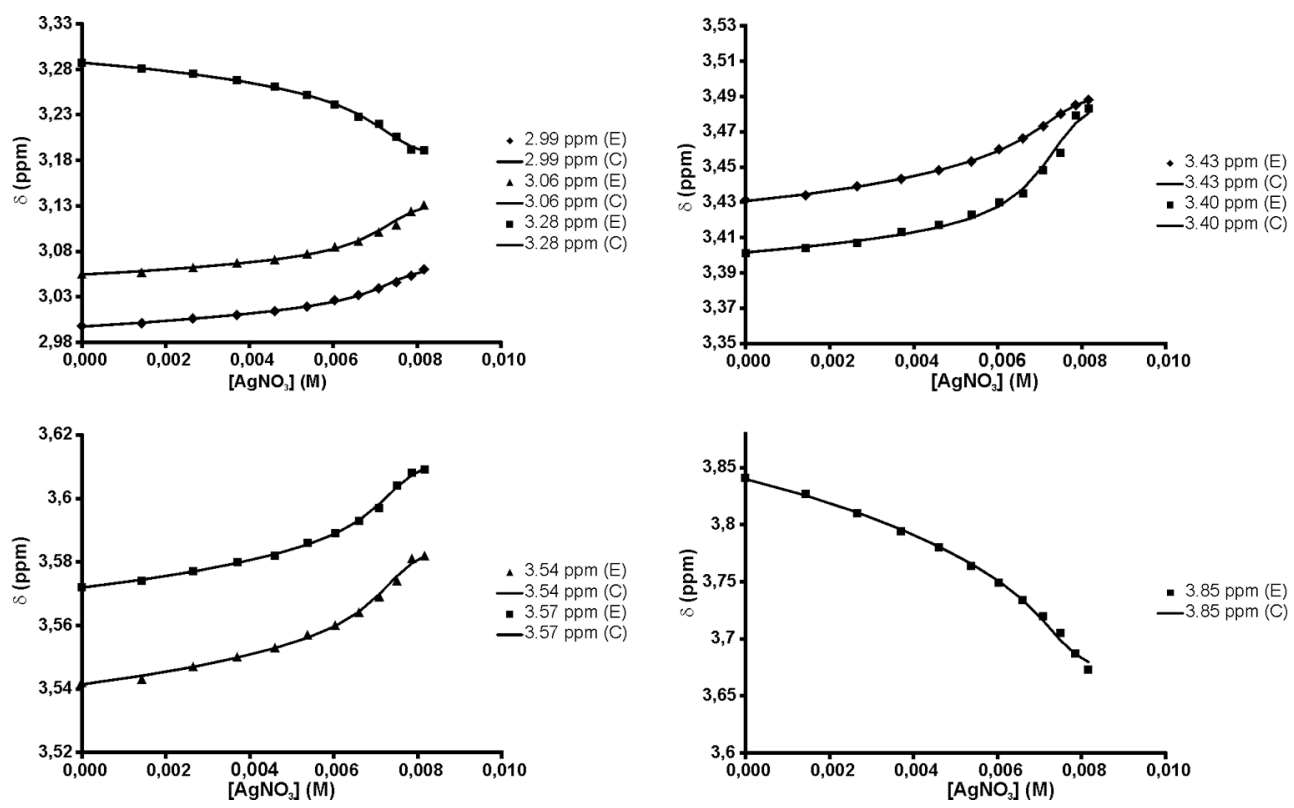

Figure 3.13. Titration curves, including experimental $(E)$ and calculated $(C)$ values, nonlinear least squares fitted to a 1:3 model assuming independent binding sites $\left(\mathrm{K}_{\mathrm{i}}=3.9 \times 10^{3} \mathrm{M}^{-1}\right)$, for the peaks $\mathrm{H}_{\mathrm{a}, \mathrm{a}, \mathrm{b}, \mathrm{c}, \mathrm{d}, \mathrm{e}, \mathrm{f}}$ of $\mathrm{AB}-\mathrm{DO} 3 \mathrm{~A}\left([\mathrm{AB}-\mathrm{DO} 3 \mathrm{~A}]_{\text {initial }}=10 \mathrm{mM}\right.$, $\left.\left[\mathrm{AgNO}_{3}\right]_{\text {added }}=10 \mathrm{mM}, 25^{\circ} \mathrm{C}\right)$ 
The titration data was successfully fitted to this 1:3 model (Figure 3.13), with $\mathrm{K}_{\mathrm{i}}=3.9 \times 10^{3} \mathrm{M}^{-1}$, strongly suggesting that at least three $\mathrm{Ag}^{+}$atoms are captured by AB-DO3A. 


\section{CHAPTER 4}

\section{SELF-ASSEMBLY TRIGGERED BY SELF-ASSEMBLY: PROTEIN CAGE ENCAPSULATED MICELLES VISUALIZED BY MRI}

\section{Abstract}

In this chapter, the successful encapsulation of paramagnetic micelles inside protein cages of the cowpea chlorotic mottle virus (CCMV) is reported. The stable assemblies formed were engineered by pure hierarchical self-assembly of micelles of the 1,4,7,10-tetraza-1-(1-carboxymethylundecane)-4,7,10-triacetic acid cyclododecane (DOTAC10) ligand inside CCMV. The DOTAC10 ligand was used to either complex with $\mathrm{Gd}^{3+}$ in order to form paramagnetic micelles visualized by $\mathrm{MRI}$ or to encapsulate amphiphilic $\mathrm{Zn}^{2+}$ phthalocyanine $(\mathrm{ZnPc})$ dye molecules, which optically confirmed the encapsulation of the micelles. Subsequent incorporation of the $\mathrm{ZnPc}$ dye in the $\mathrm{Gd}^{3+}$-DOTAC10 paramagnetic micelles led to noticeably higher capsid loadings. Protein cage formation was confirmed by transmission electron microscopy, elemental analysis and size exclusion chromatography. These purely self-assembled protein cages containing paramagnetic micelles showed enhanced ionic and capsid $r_{1}$ relaxivities, highlighting the potential of these nanostructures as contrast agents for MRI. The enhanced relaxivity properties are attributed to aggregation and altered micelle association kinetics resulting from local confinement inside the protein cage and ZnPc dye encapsulation. 


\subsection{Introduction}

Magnetic resonance imaging (MRI) is a non-invasive technique with a nonionizing character widely used to distinguish between different types of tissue while offering excellent spatial resolution., ${ }^{1,2}$ To enhance image contrast, extracellular contrast agents (CAs) based on $\mathrm{Gd}^{3+}$ complexes ${ }^{3}$ are extensively used. These CAs shorten the longitudinal relaxation times $\left(T_{1}\right)$ of the water molecules present in the tissues where they accumulate. The capability of a CA to shorten $T_{1}$, and therefore increase the signal, is expressed in terms of the relaxivity $r_{1}$.

A well-established, popular way of improving the relaxometric properties of $\mathrm{Gd}^{3+}$ complexes regards the used of amphiphilic molecules in order to make paramagnetic micelles and liposomes. ${ }^{4,5}$ The combination of high $\mathrm{Gd}^{3+}$ loadings (per aggregate) and low tumbling rates $\tau_{\mathrm{R}}$ (global rotational motion) in these self-assembled nanostructures leads to enhanced contrast resulting from increased $r_{1}$ relaxivities. ${ }^{6}$ However, a major drawback of these supramolecular aggregates is their propensity to disaggregate once below the critical aggregation concentration (cac). Yet, one way of overcoming this issue while still benefitting from relaxivity enhancement is well-illustrated by the various nanoparticles that have successfully been encapsulated in micelles and liposomes of paramagnetic amphiphiles. ${ }^{7}$

Viral protein cages have attracted much attention as potential nanometersized carriers of CAs for MRI. ${ }^{8}$ To date, the highest (ionic) relaxivity measured is attributed to paramagnetic protein cages consisting of $\mathrm{Gd}^{3+}$ ions bound to the metal binding sites of cowpea chlorotic mottle virus (CCMV) capsids. ${ }^{9}$ In order to overcome $\mathrm{Gd}^{3+}$ release from such systems, both internal and external covalent functionalization with $\mathrm{Gd}^{3+}$ complexes 
has been explored. ${ }^{10-17}$ However, covalent functionalization of viral capsids still remains a synthetically demanding task.

In this chapter, we work under the hypothesis that the undesired disaggregation of supramolecular aggregates (e.g., micelles) upon dilution can be overcome by means of confinement within CCMV capsid (i.e., protein cage without RNA inside). Herein, we describe the encapsulation of negatively charged paramagnetic micelles inside CCMV capsids by pure hierarchical self-assembly. As CCMV coat proteins (CPs) are known to self-assemble around negatively charged molecules, ${ }^{18-21}$ the negatively charged micelles are expected to trigger capsid CP self-assembly by acting as "nucleation points", yielding stable protein cages (Figure 4.1). ${ }^{22}$ MRI was used to visualize the newly formed paramagnetic capsids.

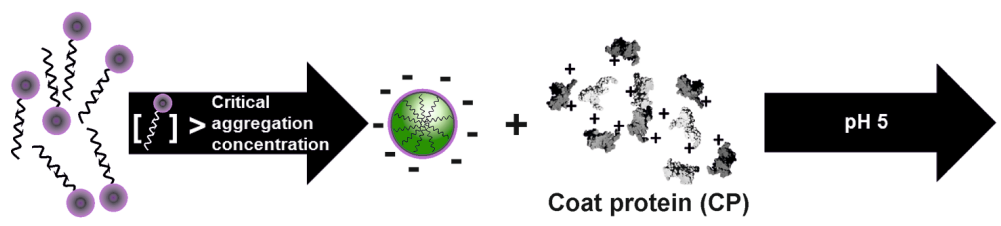

$=$ Protein cage or capsid

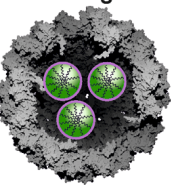

Paramagnetic capsid

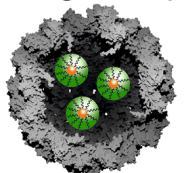

Optically active capsid

Figure 4.1. Schematic representation of the self-assembly triggered by self-assembly process: micelles are formed when the monomer concentration is higher than the critical aggregation concentration (cac). The negatively charged micelles act as "nucleation points" for self-assembly of CCMV coat proteins (CPs), yielding stable paramagnetic and optically active protein cages. The localization and orientation of the $\mathrm{ZnPc}$ molecules within the micelles is only schematic. 


\subsection{Design and Synthesis}

The building blocks of the paramagnetic micelles are $\mathrm{Gd}^{3+}$ complexes of the amphiphilic ligand 1,4,7,10-tetraza-1-(1-carboxymethylundecane)4,7,10-triacetic acid cyclododecane ( $\mathrm{Gd}^{3+}$-DOTAC10, 6), known to show enhanced $r_{1}$ relaxivities in its aggregated form (Figure 4.2). ${ }^{6}$ The (increased) local concentration effect due to cage confinement and the reduced molecular exchange of the micelles' building blocks inside the cage are envisaged to contribute to improved relaxivity properties, thereby allowing visualization by MRI. Moreover, the DOTAC10 ligand (5) was used to optically confirm encapsulation of the micelles within CCMV by means of entrapment of amphiphilic $\mathrm{Zn}^{2+}$ phthalocyanine dye molecules $(\mathrm{ZnPc})$, whose amphiphilic nature favors its inclusion into the DOTAC10 micelles.

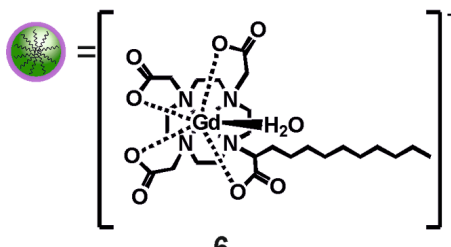

6

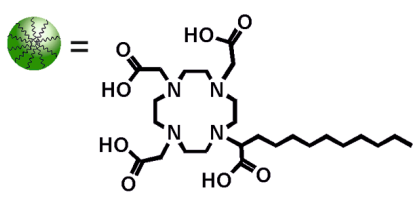

5

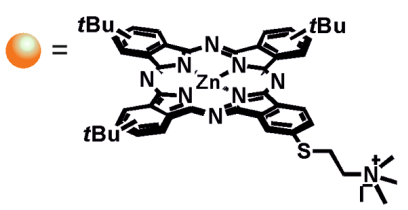

9

Figure 4.2. Chemical structure of Gd-DOTAC10 (6), DOTAC10 (5) and the amphiphilic ZnPc dye.

\subsubsection{Synthesis of the Micelles' Building Blocks}

The synthesis of the building blocks of the two types of micelles encapsulated in this study is shown in Scheme 4.1. The ligand 1,4,7,10tetraaza-1-(1-carboxymethylundecane)-4,7,10-triacetic

acid cyclododecane (DOTAC10, 5) was synthesized as follows. First, tert-butyl alcohol, 4-(dimethylamino)pyridine (DMAP), 1-ethyl-3-(3dimethylaminopropyl) carbodiimide hydrochloride (EDC) and 2- 
bromododecanoic acid (1) were allowed to react in $\mathrm{CH}_{2} \mathrm{Cl}_{2}$ for $2 \mathrm{~h}$ at $0^{\circ} \mathrm{C}$ and at room temperature overnight in order to Boc-protect the carboxylic acid of (1). Subsequently, compound (4) was obtained by alkylation of 1,4,7-tris(tert-butoxycarboxymethyl)-1,4,7,10-tetraazacyclododecane with (2) in dry acetonitrile using $\mathrm{K}_{2} \mathrm{CO}_{3}$ and $\mathrm{KI}$ (under $\mathrm{N}_{2}$ ) for three days at $50^{\circ} \mathrm{C}$. The DOTAC 10 ligand (5) was obtained by means of Bocdeprotection of (4) using $\mathrm{TFA} / \mathrm{CH}_{2} \mathrm{Cl}_{2}$ at room temperature overnight. Finally, the $\mathrm{Gd}^{3+}$ complex of DOTAC10 $\left(\mathrm{Gd}^{3+}\right.$-DOTAC10, 6) was prepared by adding one equivalent of $\mathrm{GdCl}_{3}$ to a solution of (5) in water, adjusting the $\mathrm{pH}$ to 6 and stirring at $90^{\circ} \mathrm{C}$ for 1 day. The final product (6) was obtained with an overall yield (after purification) of $13 \%$.
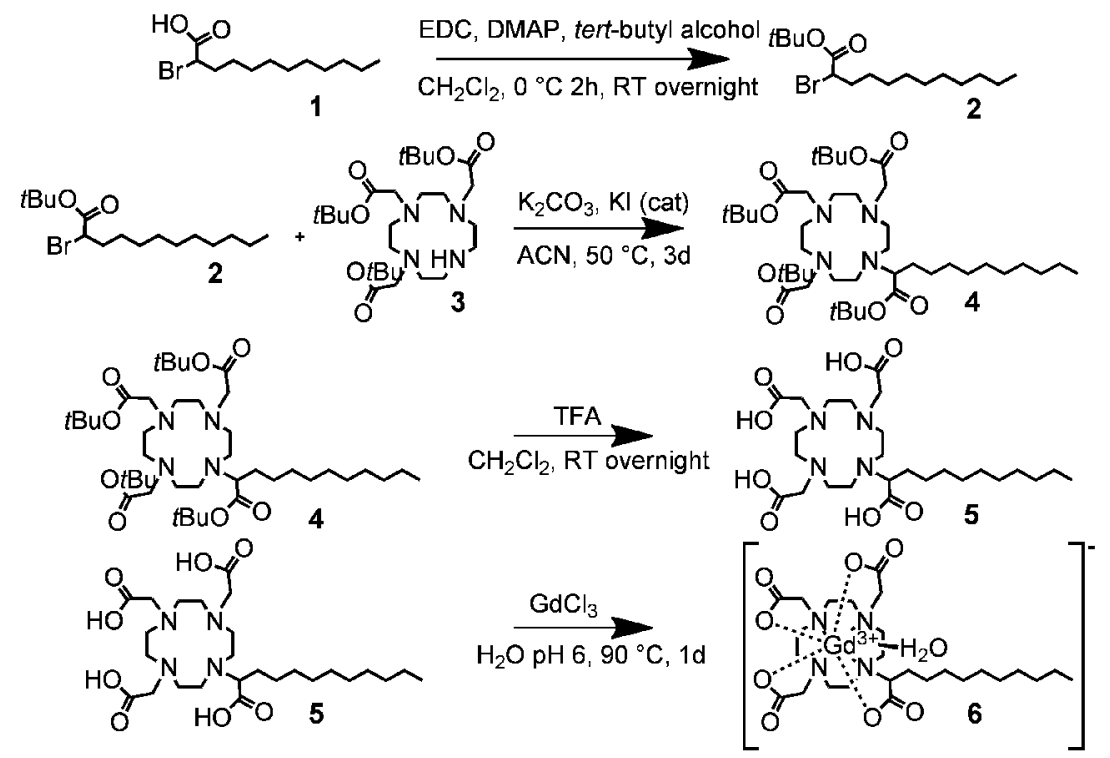

Scheme 4.1. Synthetic route followed for the synthesis of DOTAC10 (5) and its $\mathrm{Gd}^{3+}$ complex $\mathrm{Gd}^{3+}$-DOTAC10 (6). 


\subsubsection{Encapsulation in CCMV}

The building blocks of the micelles, in the form of the ligand DOTAC10 or its $\mathrm{Gd}^{3+}$ complex, were dissolved in milli-Q water to a final concentration of 0.04 M (5.5 times the $\mathrm{Cac}^{6}$ of $\mathrm{Gd}^{3+}$-DOTAC10). The "optically active" DOTAC10/ZnPc micelles were formed by addition (drop wise) of $50 \mu \mathrm{L}$ of a $5.5 \times 10^{-6} \mathrm{M} \mathrm{ZnPc}$ solution in $\mathrm{MeOH}$ to 5 . The resulting solution was heated at $80^{\circ} \mathrm{C}$ for 2 minutes, allowed to cool down and refilled with water to the initial volume. Subsequently, the $\mathrm{Gd}^{3+}$-DOTAC10 and DOTAC10/ZnPC micelle solutions were mixed with a $0.4 \mathrm{mM} \mathrm{CCMV} \mathrm{CP} \mathrm{solution}(\mathrm{pH} 7.5,50$ $\mathrm{mM}$ Tris, $300 \mathrm{mM} \mathrm{NaCl}, 1 \mathrm{mM}$ DTT) in a 1:1 ratio ( $\mathrm{v} / \mathrm{v})$. In all solutions, the final ratio was 100 monomeric micelle units per CCMV CP. The mixtures were incubated for $30 \mathrm{~min}$ at $4^{\circ} \mathrm{C}$ and dialyzed to $\mathrm{pH} 5$ for $12 \mathrm{~h}$ at the same temperature, in order to allow the CCMV CPs to self-assemble around the negatively charged micelles to form capsids. The non-encapsulated $\mathrm{ZnPc}$ molecules that precipitated out of solution were removed by centrifugation. The assembled capsids were purified by means of size exclusion chromatography (fast protein liquid chromatography, FPLC) and monitored at $\lambda=280 \mathrm{~nm}$ (characteristic of the CP).

\subsection{Results and Discussion}

\subsubsection{Evidence for Capsid Formation and Encapsulation of Micelles}

The newly formed, stable capsid assemblies eluted at $1.3 \mathrm{~mL}$, whereas unassembled CCMV CPs eluted at $2.3 \mathrm{~mL}$. In Figure 4.3a (left), which corresponds to the encapsulation of paramagnetic $\mathrm{Gd}^{3+}$-DOTAC10 micelles, the elution peak at around $1.3 \mathrm{~mL}$ is only indicative of the formation of a protein cage, as the paramagnetic micelles do not absorb in the UV region. However, successful encapsulation of DOTAC10/ZnPc 
micelles within CCMV under the previously described conditions was confirmed by the co-elution peak at $1.3 \mathrm{~mL}$ when monitoring at $\lambda=682 \mathrm{~nm}$ (Figure 4.3b, left). This absorption corresponds to encapsulated ZnPc molecules in DOTAC10 micelles (see Appendix 4.1). In addition, total reflection X-ray fluorescence (TXRF) measurements further confirmed the presence of $\mathrm{Gd}^{3+}$ and $\mathrm{Zn}^{2+}$ in both the encapsulated $\mathrm{Gd}^{3+}$-DOTAC10 and DOTAC10/ZnPc micelles, respectively (Appendix 4.2, Table 4.1 and Table 4.3).

a)

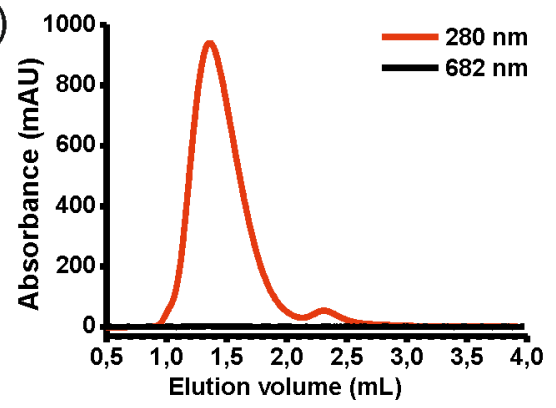

b)

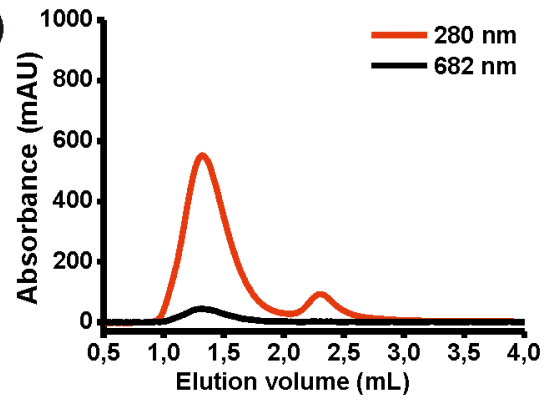

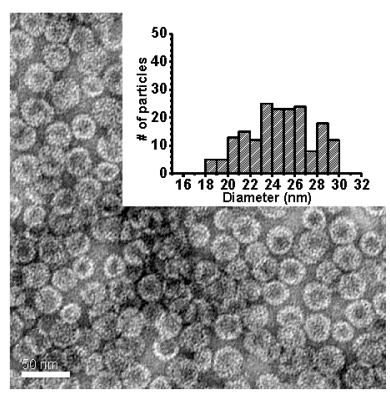

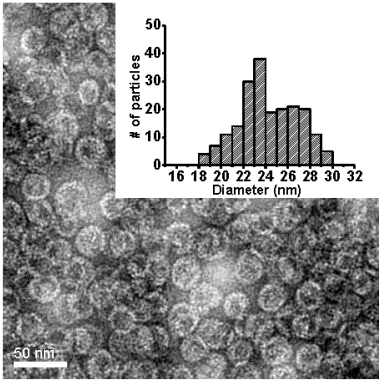

Figure 4.3. Size exclusion chromatograms (left) and transmission electron micrographs (TEM) (right) of: a) paramagnetic $\mathrm{Gd}^{3+}$-DOTAC10 micelles encapsulated in the CCMV and b) optically active DOTAC10/ZnPc micelles encapsulated in the CCMV. The red trace is characteristic of the capsid CP (monitored at $280 \mathrm{~nm}$ ), whereas the black trace is characteristic of the $\mathrm{ZnPc}$ dye (monitored at $682 \mathrm{~nm}$ ). Elution volume of the protein cages $\sim 1.3 \mathrm{~mL}$. Insets of TEM images: histograms showing the respective diameter distributions of capsids. Scale bar: $50 \mathrm{~nm}$. 
TXRF is an excellent alternative to other elemental analysis techniques (e.g., inductively coupled plasma optical emission spectroscopy ICP-OES) as it involves simple sample preparation and requires very little amounts of material. Furthermore, recent studies have demonstrated the validity of this technique for determining $\mathrm{Gd}^{3+}$ concentrations in organic matrixes (urine samples), with variable limits of detection ( $L O D=100 \mu \mathrm{g} / \mathrm{L}$ ) and quantification $(\mathrm{LOQ}=330 \mu \mathrm{g} / \mathrm{L}){ }^{23}$ While our TXRF results do confirm the presence of $\mathrm{Gd}^{3+}$ in the capsid filled with $\mathrm{Gd}^{3+}$-DOTAC10 micelles (167 $\mu \mathrm{g} / \mathrm{L}>\mathrm{LOD}$ ), quantification is not possible as the $\mathrm{Gd}^{3+}$ concentration measured is below the previously reported LOQ. Most likely, the short lifetime of the $\mathrm{Gd}^{3+}$-DOTAC10 micelles results in quick disaggregation. Since the non-aggregated monomers are lost through the pores of the capsid during dialysis, very low micelle encapsulation efficiencies are achieved. For the encapsulated DOTAC10/ZnPc micelles, where TXRF results show an average of $3 \mathrm{ZnPc}$ dye molecules/micelles hosted in a single protein cage, higher encapsulation efficiency is observed. Tentatively, the dynamics and overall charge of the micelles appear to be altered by the inclusion of the $\mathrm{ZnPc}$ dye, possibly leading to more stable, easier to encapsulate aggregates.

The formation of stable protein cages was further confirmed by transmission electron microscopy (TEM). Additionally, these images were used to determine the size distribution of the newly formed protein cages (Appendix 4.3, Table 4.5). The results showed that for the CCMV encapsulated paramagnetic $\mathrm{Gd}^{3+}$-DOTAC10 micelles, a mean size of $24.6 \pm 3 \mathrm{~nm}$ was obtained (Figure 4.3a, right). The mean size for the CCMV encapsulated optically active DOTAC10/ZnPc micelles was calculated to be $24.3 \pm 3 \mathrm{~nm}$ (Figure 4.3b, right). Both values are comparable to that of the capsid containing only DOTAC10 micelles (24.8 $\pm 3 \mathrm{~nm}$, Appendix 4.3) 
and smaller than $\mathrm{T}=3 \mathrm{CCMV}$ particles $(28 \mathrm{~nm}){ }^{24,25}$ The observed size distribution, with an average value between $20 \pm 2 \mathrm{~nm}$ ("pseudo $\mathrm{T}=2 ")^{22,26,27}$ and $28 \mathrm{~nm}(\mathrm{~T}=3)$, suggests a mixture of filled and empty capsids.

\subsubsection{Improved Properties by Means of Encapsulation of} Amphiphilic Dyes

Encouraged by the successful encapsulation of the optically active DOTAC10/ZnPc micelles, we investigated a combination of the $\mathrm{ZnPc}$ dye with the $\mathrm{Gd}^{3+}$-DOTAC10 monomers in detail (Figure 4.4a). This was done as described for DOTAC10/ZnPc micelles, but using $\mathrm{Gd}^{3+}$-DOTAC10 micelles instead, with subsequent self-assembly of the CPs around them. FPLC results confirmed the formation of a protein cage eluting at $1.3 \mathrm{~mL}$ (Figure 4.4b). However, a much lower intensity of the trace recorded at 682 $\mathrm{nm}$ was observed when compared to the case of encapsulated DOTAC10/ZnPc micelles (Figure 4.3b, left). Surprisingly, TXRF measurements showed that, on average, a protein cage assembled under these conditions contained 15 dye molecules and $350 \mathrm{Gd}^{3+}$-DOTAC10 molecules (Appendix 4.2). Assuming that the micelles formed consist of at least 15 monomers (as previously reported for $\mathrm{Gd}^{3+}$-DOTAC14 micelles), ${ }^{6}$ these data imply a maximum of 23 micelles encapsulated per capsid. Furthermore, the newly formed capsids appeared to be somewhat smaller than those containing each "functionality" alone, showing an average mean diameter of $22.4 \pm 2 \mathrm{~nm}$ (Figure 4.4c). This value is only slightly larger than the previously reported values $(20 \pm 2 \mathrm{~nm})$ corresponding to protein cages with "pseudo $T=2$ " architectures. ${ }^{22,26,27}$ Given the previously discussed assumption that a mixture of both "pseudo $T=2$ " and $T=3$ particles are formed after the encapsulation of $\mathrm{Gd}^{3+}$-DOTAC10 and DOTAC10/ZnPc micelles, the average mean diameter obtained for the 
$\mathrm{Gd}^{3+}$-DOTAC10/ZnPc loaded micelles points out to the formation of more "pseudo $\mathrm{T}=2$ " particles than in the former cases.

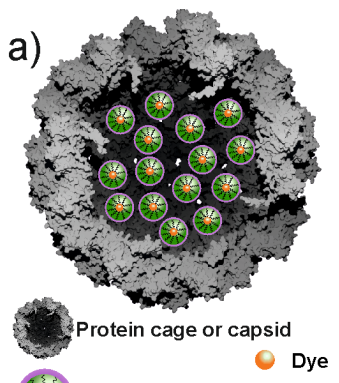

Gd-DOTAC10 micelles
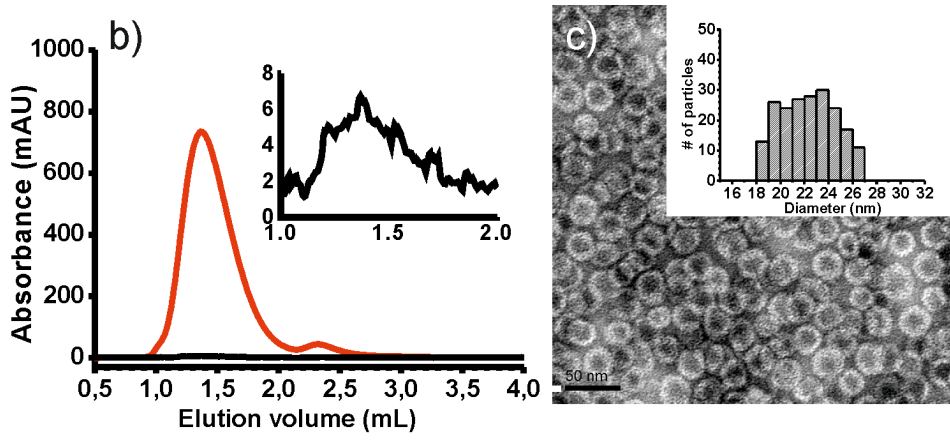

Figure 4.4. a) Schematic representation of the CCMV capsid containing $\mathrm{Gd}^{3+}$ DOTAC10/ZnPc micelles. b) Size exclusion chromatogram. The red trace is characteristic of the capsid CP (monitored at $280 \mathrm{~nm}$ ), whereas the black trace is characteristic of the $\mathrm{ZnPc}$ dye (monitored at $682 \mathrm{~nm}$ ). Elution volume of the protein cages $\sim 1.3 \mathrm{~mL}$. c) TEM image. Inset: Histogram showing the diameter distribution of capsids filled with $\mathrm{Gd}^{3+}-$ DOTAC10/ZnPc micelles. Scale bar: $50 \mathrm{~nm}$.

Noticeably, the initial observation suggesting that the encapsulation efficiency of DOTAC10-based micelles is affected by the presence of the ZnPc dye coincides with the previously presented TEM and TXRF data. Although our EM studies do not provide conclusive data on the actual filled/empty capsid ratio, the smaller size distribution found for the $\mathrm{Gd}^{3+}$ DOTAC10/ZnPc encapsulated micelles (Figure 4.4c), together with the significantly higher $\mathrm{Gd}^{3+}$ loadings found by TXRF analysis, points towards a considerably improved filled/empty capsid ratio. Moreover, TXRF confirmed the presence of the $\mathrm{ZnPc}$ dye and the affected optical properties observed upon encapsulation in $\mathrm{Gd}^{3+}$-DOTAC10 micelles might be a consequence of $\mathrm{ZnPc}$ dye stacking inside the micelles. Therefore, either a micelle consists of more than $15 \mathrm{Gd}^{3+}$-DOTAC10 monomers, or the 
$\mathrm{ZnPc}$ dye encapsulation efficiency of the micelles varies. Altogether, the encapsulation of the $\mathrm{ZnPc}$ dye seems to lead to a significant change in the overall surface charge of the $\mathrm{Gd}^{3+}$-DOTAC10 micelles, which might be responsible for promoting a stronger interaction between the $\mathrm{Gd}^{3+}$ DOTAC10 micelle surface and the CP, leading to smaller structures with (clearly) higher loadings.

\subsubsection{Paramagnetic Properties of the Encapsulated Micelles}

The encapsulation of paramagnetic micelles was confirmed by determining their relaxation efficiency using MRI. Here, the longitudinal relaxation times $\left(T_{1}\right)$ of a series of dilutions (of known $\mathrm{Gd}^{3+}$ and capsid concentrations) were measured by means of a standard saturation recovery experiment at 600 $\mathrm{MHz}(14.1 \mathrm{~T})$. By plotting the measured $1 / T_{1}$ values as a function of $\mathrm{Gd}^{3+}$ or capsid concentration, the relaxivity $r_{1}\left(\mathrm{mM}^{-1} \mathrm{~s}^{-1}\right)$ was obtained as the slope of the curve (Appendix 4.4). For comparison purposes, the ionic $r_{1}$ of a 45 $\mu \mathrm{M}$ solution of the free $\mathrm{Gd}^{3+}$-DOTAC10 complex $\left(\left[\mathrm{Gd}^{3+}\right.\right.$-DOTAC10] < cac) was also calculated (2.52 $\mathrm{mM}^{-1} \mathrm{~s}^{-1}$, Figure 4.5). Owing to the impossible quantification of the $\mathrm{Gd}^{3+}$ concentration of the encapsulated paramagnetic $\mathrm{Gd}^{3+}$-DOTAC10 micelles, any changes in the global rotational motion of $\mathrm{Gd}^{3+}$-DOTAC10 monomers upon micellization inside the capsid are not properly assessable. 


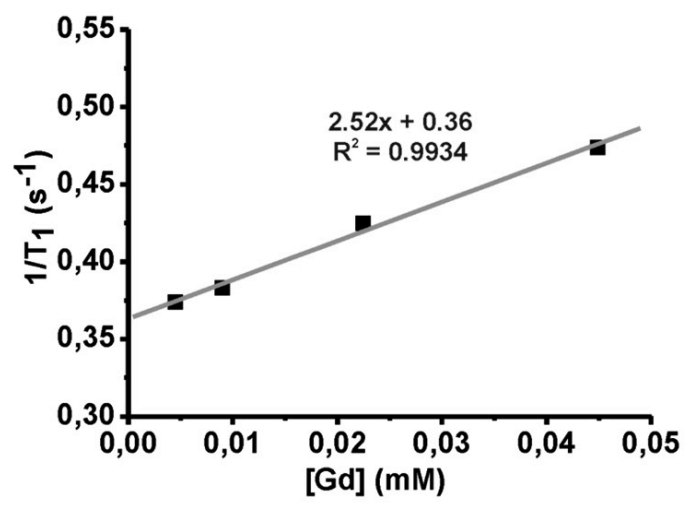

Figure 4.5. Relaxivity plots at $600 \mathrm{MHz}$ and $25^{\circ} \mathrm{C}$ of the $\mathrm{Gd}^{3+}$-DOTAC10 complex (6, $\left.r_{1}=2.52 \mathrm{mM}^{-1} \mathrm{~s}^{-1}\right)$.

A decrease in the tumbling rate $\tau_{R}$ of the complex due to micellization inside the capsid ${ }^{4-6}$ is confirmed by the calculated ionic $r_{1}$ value for the $\mathrm{Gd}^{3+}$-DOTAC10/ZnPc encapsulated micelles (3.85 $\mathrm{mM}^{-1} \mathrm{~s}^{-1}$, Figure 4.6a), which is higher than that obtained for the free $\mathrm{Gd}^{3+}$-DOTAC10 complex in solution $\left(2.52 \mathrm{mM}^{-1} \mathrm{~s}^{-1}\right)$. This ionic $r_{1}$ value is comparable to previously reported values (measured at high magnetic field strengths) involving covalently attached $\mathrm{Gd}^{3+}$-DOTA complexes to wild-type cowpea mosaic virus (CPMV, 3-4.4 $\mathrm{mM}^{-1} \mathrm{~s}^{-1}$ at $500 \mathrm{MHz}$ ). ${ }^{15}$ Nevertheless, making a fair comparison between our "paramagnetic capsid" and previously reported values $^{15}$ is not straightforward as the enhancement shown by the latter systems is related to the slower tumbling rate of the $\mathrm{Gd}^{3+}$ complexes due to covalent attachment to the protein cage. In our case, as the micellar structures are (non-covalently) encased inside the capsid, we believe that the differences in relaxivity can be attributed to the altered dynamics and molecular exchange kinetics of the micelle monomers as a result of capsid confinement (e.g., decreased intermicellar distance). ${ }^{30,3128,29}$ Moreover, the dynamic behavior of the $\mathrm{Gd}^{3+}$-DOTAC10 micelles might vary as the micelle 
robustness and properties change upon encapsulation of the $\mathrm{ZnPc}$ dye. Likewise, the higher flexibility of the non-covalently attached paramagnetic moieties can also contribute to high relaxivity values, as recent studies suggest that (at high magnetic fields) some degree of flexibility of the $\mathrm{Gd}^{3+}$ chelate is vital. ${ }^{30}$
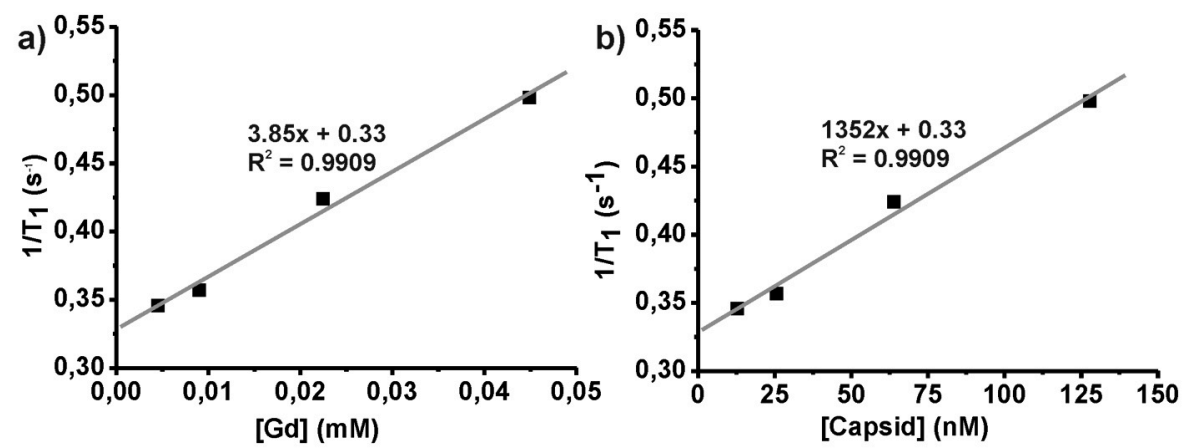

Figure 4.6. Relaxivity plot of capsid filled with $\mathrm{Gd}^{3+}$-DOTAC10/ZnPc micelles at $600 \mathrm{MHz}$ and $25^{\circ} \mathrm{C}$ : a) based on $\mathrm{Gd}^{3+}$ concentration $\left(r_{1}=3.85 \mathrm{mM}^{-1} \mathrm{~s}^{-1}\right)$ and b) based on capsid concentration $\left(r_{1}=1352 \mathrm{mM}^{-1} \mathrm{~s}^{-1}\right)$.

With respect to the capsid $r_{1}$ value for encapsulated $\mathrm{Gd}^{3+}$-DOTAC10/ZnPc micelles (1352 $\mathrm{mM}^{-1} \mathrm{~s}^{-1}$, Figure 4.6b), the observed enhancement is attributed to the high local $\mathrm{Gd}^{3+}$ concentration (confirmed by TXRF) that can be achieved upon encapsulation. Owing to the high amount of $\mathrm{Gd}^{3+}$ encapsulated, a decreased ionic $r_{1}$ relaxivity is expected as studies on $\mathrm{Gd}^{3+}$-chelate micellar systems have shown that decreased distances between $\mathrm{Gd}^{3+}$-monomers affect the electronic relaxation times. ${ }^{31}$ Therefore, although we are not able to quantify the $\mathrm{Gd}^{3+}$ payload of the protein cage encapsulated $\mathrm{Gd}^{3+}$-DOTAC10 micelles, their $r_{1}$ value must be higher than $3.85 \mathrm{mM}^{-1} \mathrm{~s}^{-1}\left(\mathrm{Gd}^{3+}\right.$-DOTAC10/ZnPc micelles). 


\subsubsection{Visualization of Encapsulated Micelles by MRI}

The encapsulation of paramagnetic $\mathrm{Gd}^{3+}$-DOTAC10 and $\mathrm{Gd}^{3+}$ DOTAC10/ZnPc micelles is illustrated in the $T_{1}$-weighted MR image shown in Figure 4.7. The aggregation inside the capsid of the paramagnetic micelles is demonstrated by the higher contrast both show at low concentrations of $\mathrm{Gd}^{3+}$ and far below the cac of $\mathrm{Gd}^{3+}$-DOTAC10 in solution. ${ }^{6}$ Also, the encapsulation of paramagnetic Gd-DOTAC10 micelles ( $<1.1 \mu \mathrm{M} \mathrm{Gd}^{3+}$ concentration) is evidenced by the higher contrast it shows when compared to $\mathrm{Gd}^{3+}$-DOTAC10 in solution at higher concentrations (45 $\mu \mathrm{M} \mathrm{Gd}^{3+}$ ) and their differences in signal-to-noise ratio (83 vs. 65, respectively).

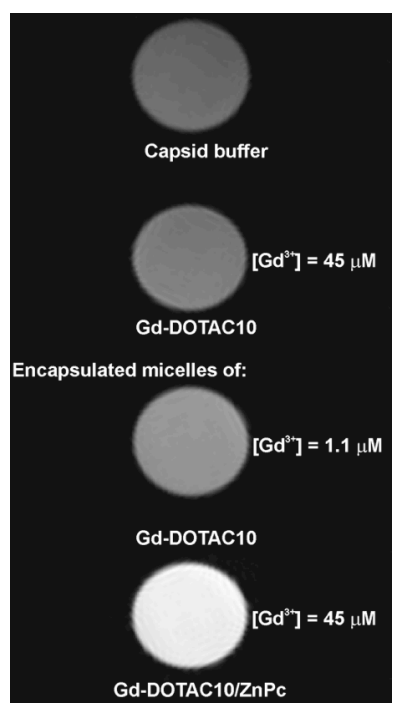

Figure 4.7. $T_{1}$-weighted $\mathrm{MR}$ image of encapsulated paramagnetic $\mathrm{Gd}^{3+}$-DOTAC10 and $\mathrm{Gd}^{3+}$-DOTAC10/ZnPC micelles at $600 \mathrm{MHz}$ (TE $=6 \mathrm{~ms}$, TR $\left.=500 \mathrm{~ms}\right)$. For direct comparison, the contrast given by capsid buffer $(50 \mathrm{mM}$ sodium acetate, $1 \mathrm{M}$ sodium chloride and $1 \mathrm{mM}$ sodium azide, $\mathrm{pH}$ 5) and a solution containing $\mathrm{Gd}^{3+}$-DOTAC10, at the highest $\mathrm{Gd}^{3+}$ concentration measured $(45 \mu \mathrm{M})$, are also shown. 
Whereas the encapsulation of $\mathrm{Gd}^{3+}$-DOTAC10 micelles in CCMV is clearly less efficient, inclusion of the $\mathrm{ZnPc}$ dye in these micelles leads to improved ionic $r_{1}$ relaxivities and high capsid loadings, as shown by the MR image in Figure 4.7.

\subsection{Conclusions}

In summary, we report the MRI visualization of encapsulated paramagnetic micelles inside protein cages. $T_{1}$-weighted $\mathrm{MR}$ images and relaxivity curves showed that, upon encapsulation of $\mathrm{Gd}^{3+}$-DOTAC10 micelles within the CCMV capsid, enhanced $r_{1}$ values are obtained. This enhancement is attributed to i) aggregation resulting from local confinement inside the protein cage and ii) altered micelle association kinetics probably due to a decreased intermicellar distance. The higher payloads, altered size distribution and clear enhancement of the ionic $r_{1}$ values of encapsulated $\mathrm{Gd}^{3+}$-DOTAC10/ZnPc micelles show that the encapsulation of these "combined" micelles is more successful than that of only $\mathrm{Gd}^{3+}$-DOTAC10 micelles. Furthermore, these paramagnetic protein cages might show therapeutically relevant properties, since $\mathrm{ZnPc}$ dyes are known for their use in photodynamic therapy. ${ }^{18,32,33}$ Our approach is based on hierarchical self-assembly and offers a simple, fast, synthetically straightforward methodology that can be extended to micelles with other dyes for (multimodal) optical imaging. While little is known about the behavior of these new protein cages at physiological $\mathrm{pH}$, the present work is a first step towards the potential use of self-assembled nanocages in vivo. 


\subsection{Experimental}

Materials. Commercial grade reagents were purchased from Sigma Aldrich and $\mathrm{TCl}$ Europe and were used without further purification unless otherwise stated. Solvents were dried over molecular sieves. Deuterated solvents were purchased from Cambridge Isotope Laboratories and NMR and MRI tubes from Wilmad LabGlass. All chemicals used for the preparation of buffers were of analytical quality and were prepared in ultrapure (Milli-Q) water.

2-Bromododecanoic acid tert-butyl ester (2). This compound was synthesized following an adapted procedure. ${ }^{34}$ 2-bromododecanoic acid (1) $(2 \mathrm{~g}, 7.1 \mathrm{mmol}), 4$-(dimethylamino)pyridine $(0.44 \mathrm{~g}, 3.6 \mathrm{mmol})$ and tertbutanol $(0.6 \mathrm{~g}, 8.1 \mathrm{mmol})$ were dissolved in $40 \mathrm{~mL}$ dry $\mathrm{CH}_{2} \mathrm{Cl}_{2}$ and cooled in an ice bath. Subsequently, I-ethyl-3-(3-dimethylaminopropyl) carbodiimide hydrochloride $(1.5 \mathrm{~g}, 7.8 \mathrm{mmol})$ was added and the reaction mixture was stirred at $0^{\circ} \mathrm{C}$ for $2 \mathrm{~h}$ and at room temperature overnight. The solution was washed with saturated sodium bicarbonate $(2 \times 60 \mathrm{~mL})$, water $\left(3 \times 60 \mathrm{~mL}\right.$ ), and dried over $\mathrm{MgSO}_{4}$. The organic phase was passed through a layer of silica and the solvent was removed under reduced pressure. The desired compound was obtained as a colorless liquid after chromatography on silica gel 60 (hexane/EtOAc 20:1, later 9:1). Yield: $34 \%$ (810 mg, $2.4 \mathrm{mmol}) .{ }^{1} \mathrm{H}$ NMR (600 MHz, $\left.\mathrm{CDCl}_{3}, 25^{\circ} \mathrm{C}, \mathrm{TMS}\right): \delta$ (ppm):

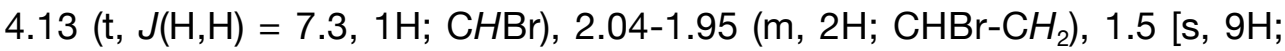
$\left.\mathrm{C}\left(\mathrm{CH}_{3}\right)_{3}\right], 1.28\left(\mathrm{~m}, 16 \mathrm{H} ; \mathrm{CH}_{2}\right), 0.9\left(\mathrm{t}, \mathrm{J}(\mathrm{H}, \mathrm{H})=6.8,3 \mathrm{H} ; \mathrm{CH}_{3}\right) .{ }^{13} \mathrm{C}$ NMR $(150$ $\left.\mathrm{MHz}, \mathrm{CDCl}_{3}, 25^{\circ} \mathrm{C}, \mathrm{TMS}\right) \delta$ (ppm): 169 (COOtBu), $82.2\left[\mathrm{C}\left(\mathrm{CH}_{3}\right)_{3}\right], 47.9$ $(\mathrm{HCBr}), 34.9\left(\mathrm{HCBr}-\mathrm{CH}_{2}\right), 31.9\left(\mathrm{HCBr}-\mathrm{CH}_{2}-\mathrm{CH}_{2}\right), 29.5,29.5,29.4$, 29.3, 
28.9, 27.3, $22.7\left(\mathrm{CH}_{2}\right)$, $27.8\left[\mathrm{C}\left(\mathrm{CH}_{3}\right)_{3}\right], 14.1\left(\mathrm{CH}_{3}\right) . \mathrm{MS}\left(\mathrm{ESI}+, \mathrm{CH}_{2} \mathrm{Cl}_{2} / \mathrm{MeOH}\right)$ : $\mathrm{m} / \mathrm{z}: 257.5[\mathrm{M}-\mathrm{Br}+\mathrm{H}]^{+}, 301.6$, (fragm), 311.8 (fragm), $357.8[\mathrm{M}+\mathrm{Na}]^{+}$.

\section{1,4,7,10-Tetraaza-1-(1-carboxy-tert-butoxymethylundecane)-}

4,7,10-triacetic acid tert-butyl ester cyclododecane (4). In a threeneck round-bottom flask, $\mathrm{K}_{2} \mathrm{CO}_{3}(370 \mathrm{mg}, 2.7 \mathrm{mmol})$ and $\mathrm{KI}$ (catalytic amount) were added to a stirring solution of 1,4,7-tris(tertbutoxycarboxymethyl)-1,4,7,10-tetraazacyclododecane 3 (DO3A, synthesized as previously described ${ }^{35}$ ) $(460 \mathrm{mg}, 0.9 \mathrm{mmol})$ in $40 \mathrm{~mL}$ dry acetonitrile. The mixture was kept under $\mathrm{N}_{2}$ for 10 minutes under vigorous stirring. Afterwards, 2-bromododecanoic acid tert-butyl ester (2) (300 mg, $0.9 \mathrm{mmol}$ ) dissolved in $10 \mathrm{~mL}$ dry $\mathrm{CH}_{2} \mathrm{Cl}_{2}$ was added slowly to the reaction mixture which was, subsequently, allowed to react for 3 days at $50^{\circ} \mathrm{C}$. The mixture was left to cool down, filtered to remove inorganic salts and the solvent was evaporated under reduced pressure. The desired compound was obtained as a yellow oil after chromatography on silica $\left(\mathrm{CH}_{2} \mathrm{Cl}_{2} / \mathrm{EtOH}\right.$ 9:1, later $\mathrm{EtOH} / \mathrm{NH}_{3}$ 95:5). Yield: $37 \%$ (253 mg, $\left.0.3 \mathrm{mmol}\right) .{ }^{1} \mathrm{H}$ NMR (600 $\left.\mathrm{MHz}, \mathrm{CDCl}_{3}, 25^{\circ} \mathrm{C}, \mathrm{TMS}\right) \delta(\mathrm{ppm}): 3.67$ (dd, $\left.1 \mathrm{H} ; \mathrm{CHN}\right), 3.5-2(\mathrm{~m}, 22 \mathrm{H} ; \mathrm{CHN}-$ $\left.\mathrm{CH}_{2}, \mathrm{CH}_{2}-\mathrm{COOtBu}\right), 1.58\left(\mathrm{~m}, 2 \mathrm{H} ; \mathrm{CHN}-\mathrm{CH}_{2}\right), 1.46-1.44\left(\mathrm{~s}, 36 \mathrm{H} ; \mathrm{C}\left(\mathrm{CH}_{3}\right)_{3}\right)$, $1.25\left(\mathrm{~m}, 16 \mathrm{H} ; \mathrm{CH}_{2}\right), 0.88\left(\mathrm{t}, \mathrm{J}(\mathrm{H}, \mathrm{H})=6.9,3 \mathrm{H} ; \mathrm{CH}_{3}\right) .{ }^{13} \mathrm{C} \mathrm{NMR}(150 \mathrm{MHz}$, $\mathrm{CDCl}_{3}, 25^{\circ} \mathrm{C}$, TMS) $\delta(\mathrm{ppm}): 173,172.9,(\mathrm{COOtBu}), 81.6,81.7\left[\mathrm{C}\left(\mathrm{CH}_{3}\right)_{3}\right]$, $70.6\left(\mathrm{HCNCH}_{2}\right), 61.2,55.9,55.6,55.3,53.4,52.5,48.9,48.5,47.3,45$ $\left(\mathrm{HCNCH}_{2}, \mathrm{NCH}_{2}, \mathrm{CH}_{2} \mathrm{COOtBu}\right), 31.9,31.92,31,29.7,29.7,29.6,29.4$, 29.4, 29.3, 29.1, 27.94, 27.92, 27.9, $27.8\left[\mathrm{CH}_{2}, \mathrm{C}\left(\mathrm{CH}_{3}\right)_{3}\right], 14.1\left(\mathrm{CH}_{3}\right)$. MS (ESI+, $\left.\mathrm{CH}_{2} \mathrm{Cl}_{2} / \mathrm{MeOH}\right): \mathrm{m} / \mathrm{z}: 791.4\left[\mathrm{M}^{+}+\mathrm{Na}\right]$.

\section{1,4,7,10-Tetraaza-1-(1-carboxymethylundecane)-4,7,10-triacetic acid cyclododecane (DOTAC10, 5). In a round-bottom flask, TFA}


(4 mL, $52 \mathrm{mmol}$ ) was added to a solution of 4 (100 $\mathrm{mg}, 0.13 \mathrm{mmol})$ in $4 \mathrm{~mL}$ $\mathrm{CH}_{2} \mathrm{Cl}_{2}$ and the reaction mixture was allowed to stir at room temperature overnight. The mixture was evaporated under reduced pressure and the residue co-evaporated with $\mathrm{CH}_{2} \mathrm{Cl}_{2} 3$ times in order to remove excess TFA. 5 was obtained as a solid after precipitation from diethyl ether and it was subsequently used for the next reaction. Yield: $98 \%$ (69 $\mathrm{mg}, 0.127 \mathrm{mmol}$ ). MS (ESI+, $\left.\mathrm{H}_{2} \mathrm{O} / \mathrm{ACN}+0.1 \% \mathrm{TFA}\right): \mathrm{m} / \mathrm{z}: 545.4\left[\mathrm{M}^{+}+\mathrm{H}\right]$.

\section{Gd $^{3+}-1,4,7,10-T e t r a a z a-1-(1-c a r b o x y m e t h y l u n d e c a n e)-4,7,10-$}

triacetic acid cycloddodecane (Gd ${ }^{3+}$-DOTAC10, 6). In a roundbottom flask, 5 (40 mg, $0.073 \mathrm{mmol}$ ) and $\mathrm{GdCl}_{3}(19 \mathrm{mg}, 0.073 \mathrm{mmol}$ ) were dissolved in $10 \mathrm{~mL}$ water. The $\mathrm{pH}$ of the mixture was adjusted to 6 using $\mathrm{NaOH}(1 \mathrm{M})$ and the reaction mixture was allowed to stir at $90^{\circ} \mathrm{C}$ overnight. The solution was allowed to cool for 30 minutes and it was subsequently stirred for 10 minutes with (previously washed with acetone) DOWEX Monosphere $650 \mathrm{C}$ cation exchange resin. After filtering in order to remove the resin, the absence of free $\mathrm{Gd}^{3+}$ ions was confirmed by the xylenol orange test. Compound $\mathbf{6}$ was obtained after the solvent was evaporated under reduced pressure. Yield: 99\% (50 mg, $0.072 \mathrm{mmol}$ ). MS (MALDI-, $\beta$-indoleacrylic acid ${ }^{36}$ ): $\mathrm{m} / \mathrm{z}$ : 698.217 [M-]. The BMS shift for a $100 \mu \mathrm{M}$ of $\mathrm{Gd}^{3+}$-DOTAC10 was $0.012 \mathrm{ppm}$ (theoretical value $=0.0109 \mathrm{ppm}$ ).

Zinc (II) $\quad 2(3), 9(10), 16(17)$-tri-tert-butyl-23 (24)-(2trimethylaminoethylsulfanyl) phthalocyaninato)(ฉ-)-N29, $\mathbf{N}^{30}$, $\mathbf{N}^{31}, \mathbf{N}^{32}$ iodide (ZnPc dye). PhD thesis of Eduardo Anaya-Plaza, Universidad Autónoma de Madrid. 
CCMV Synthesis and Characterization. Purification of CCMV, removal of RNA and isolation of the CP were carried out according to literature procedures. $^{37,38}$ The purity of the viral CP was characterized by fast protein liquid chromatography (FPLC; elution volume of $\mathrm{CP}=2.3 \mathrm{~mL}$ ) and UV-Vis absorbance spectroscopy.

Nuclear Magnetic Resonance (NMR) Characterization. All NMR experiments were performed on a Bruker Avance II NMR spectrometer. Chemicals shifts are given in ppm using the residual solvent signal as reference. The multiplicity of the peaks is reported using the following abbreviations: $\mathrm{s}=$ singlet, $\mathrm{d}=$ doublet, $\mathrm{t}=$ triplet, $\mathrm{q}=$ quartet, $\mathrm{m}=$ multiplet. All NMR spectra were processed using TOPSPIN 2.1 and MestReC software packages. Quality of the $\mathrm{Gd}^{3+}$-DOTAC10 complex was assessed by measuring the Bulk Magnetic Susceptibility (BMS) shift $\left(\Delta_{x}\right)$ of a $100 \mu \mathrm{M}$ solution of the complex (measured according to literature procedures $^{39}$ ) and comparing it to the theoretical BMS shift given by:

$$
\Delta_{x}=\frac{4 \pi c s}{T}\left(\frac{\mu_{e f f}}{2.84}\right)^{2} \times 10^{3}
$$

Where $\mathrm{c}$ is the concentration of the paramagnetic solute in $\mathrm{mol} / \mathrm{L}$, $\mathrm{s}$ is dependent on the shape of the sample $(s=1 / 3$ for a cylinder parallel to the main field), $T$ is the absolute temperature (in $\mathrm{K}$ ) and $\mu_{\text {eff }}$ is the effective magnetic moment for $\mathrm{Gd}^{3+}\left(7.94^{40}\right)$.

Mass Spectrometry (MS). Mass spectra were acquired on a Waters Micromass LCT ESI mass spectrometer, an Applied Biosystems QSTAR ESI mass spectrometer, a Bruker Ultraflex III MALDI-TOF mass spectrometer and a Voyager-DE RP mass spectrometer. 
UV-Vis Spectroscopy. UV spectra were acquired on a Perkin Elmer Lambda 850 UV-Vis spectrometer, a Jasco V-660 spectrometer and a NanoDrop 1000 spectrophotometer from Thermo Scientific.

Fast Protein Liquid Chromatography (FPLC). Size exclusion chromatography was performed using the following buffers: $50 \mathrm{mM}$ sodium acetate, $1 \mathrm{M}$ sodium chloride and $1 \mathrm{mM}$ sodium azide $(\mathrm{pH} 5)$. The FPLC was equipped with a Superose 6 size exclusion column 10/100 GL (GE Healthcare) with a bed volume of $2.4 \mathrm{~mL}$. For each run, the injection volume was $100 \mu \mathrm{L}$.

Transmission Electron Microscopy (TFM). TEM images were obtained using analytical TEM FEI instruments. In order to prepare the samples, $5 \mu \mathrm{L}$ of the desired sample was applied onto Formvar-carbon coated grids (from electron microscopy sciences). After leaving the sample for 1 minute, the excess of liquid was removed using a piece of filter paper. Uranyl acetate $(5 \mathrm{~mL}, 1 \% \mathrm{w} / \mathrm{v})$ was then applied and the drying procedure was repeated. The size distribution of capsids was determined by measuring their diameter, for more than 200 counts, from TEM images.

Total Reflection X-ray Fluorescence (TXRF). TXRF measurements were performed by a Bruker S2 Picofox TXRF (Bruker AXS GmbH, Karlsruhe, Germany) portable system. The primary $X$ rays were generated by an air-cooled low power X-ray tube using a molybdenum anode. The excitation settings were $50 \mathrm{kV}$ and $750 \mathrm{~mA}$. Measurements were performed by signal integration over 1500 seconds. Quartz glass disks were used as sample carriers, which were previously cleaned in $1 \mathrm{~N}$ nitric 
acid followed by flushing in water for $1 \mathrm{~h}$ at $80^{\circ} \mathrm{C}$. After that, they were dried, coated with silicon solution for better wettability and dried again. Before measuring the samples, the quartz glass sample carriers were analyzed to ensure the absence of $\mathrm{Gd}^{3+}$ contamination. The quantification is performed by the Bruker S2 Picofox TXRF software (Spectra, Version 6.1.5.0), based on the known quantity of the gallium internal standard added. Samples for TXRF analysis were prepared by adding equal amounts of the gallium internal standard $(10 \mathrm{mg} / \mathrm{L})$ and the sample to be analyzed (volume). After vortexing, a drop of the solution was placed on the quartz disk and dried under vacuum for 15 minutes. For control purposes, elemental analysis of the empty capsid (in buffer) was conducted and the values obtained, for each capsid sample in question, were corrected.

Magnetic Resonance Imaging (MRI). All experiments were carried out on a $600 \mathrm{MHz}(14.1 \mathrm{~T})$ Avance II NMR Spectrometer from Bruker (Karlsruhe, Germany) equipped with a vertical narrow bore magnet (14.1 $T)$, a great $B_{0}$ compensation unit (BGU-II) and 3 great 1/60 amplifier units $(X, Y$ and $Z$ ) also from Bruker. A micro 5 imaging probe, equipped with a $10 \mathrm{~mm}$ diameter saddle coil insert from Bruker (Karlsruhe, Germany) was used. The maximum gradient strength was $4.8 \mathrm{G} / \mathrm{cm} / \mathrm{A}$ and the temperature inside the probe was kept at $\sim 25^{\circ} \mathrm{C}$ by a gradient water cooling unit BCU20 from Bruker. All NMR/MRI tubes (5/10 mm diameter) were purchased from Wilmad Labglass (New Jersey, U.S.A.). Glass capillary tubes (melting point tubes, inner diameter $1 \mathrm{~mm}$ ) used for MRI were purchased from VWR. $T_{1}$ measurements were performed in a $1 \mathrm{~cm}$ (outer diameter) glass tube as follows. Stock solutions of the $\mathrm{Gd}^{3+}$-DOTA complex and the encapsulated $\mathrm{Gd}^{3+}$-DOTA and $\mathrm{Gd}^{3+}$-DOTA/ZnPc micelles (with known concentrations of $\mathrm{Gd}^{3+}$ and capsid) were diluted 2, 5 and 10 
times. For each experiment, capillary tubes of $1 \mathrm{~mm}$ inner diameter were filled with these solutions, placed in a custom-made Kel-F capillary holder and subsequently introduced in the $1 \mathrm{~cm}$ tube for imaging. Longitudinal relaxation times were measured using a saturation recovery pulse sequence with static TE $=6 \mathrm{~ms}$ and 16 TR values, namely 100, 150, 200, $350,500,600,700,800,1000,1500,2000,3000,4000,6000,8000,10000$ $\mathrm{ms}$. The measurements were done on 5 subsequent axial slices of $1 \mathrm{~mm}$, with a field of view (FOV) of $10 \mathrm{~mm} \times 10 \mathrm{~mm}$, a matrix size of $128 \times 128$ and 2 averages. $T_{1}$ values were obtained by mono-exponentially fitting the intensities of selected regions of interest (ROIs) for each axial slice using the image sequence analysis tool in Paravision 4.0 software. $T_{1}$-weighted images were obtained using a spin echo sequence with the following parameters: TR $=500 \mathrm{~ms}, \mathrm{TE}=6 \mathrm{~ms}, 1$ axial slice of $6 \mathrm{~mm}$ thickness, FOV $=10 \mathrm{~mm} \times 10 \mathrm{~mm}$, matrix size $=256 \times 256$ and 10 averages. The longitudinal relaxivity $r_{1}$ (in $\mathrm{mM}^{-1} \mathrm{~s}^{-1}$ ) was obtained as the slope of the plot $1 / T_{1}$ vs. $\mathrm{Gd}^{3+}$ (or capsid) concentration:

$$
\left(\frac{1}{T_{1}}\right)_{o b s}=\left(\frac{1}{T_{1}}\right)_{d}+r_{1}[G d]
$$

\subsection{References}

(1) Cassidy, P. J.; Radda, G. K. J. Royal Soc. Interface. 2005, 2, 133.

(2) Weissleder, R.; Mahmood, U. Radiology 2001, 219, 316.

(3) Caravan, P.; Ellison, J. J.; McMurry, T. J.; Lauffer, R. B. Chem. Rev. 1999, 99, 2293.

(4) Accardo, A.; Tesauro, D.; Aloj, L.; Pedone, C.; Morelli, G. Coord. Chem. Rev. 2009, 253, 2193.

(5) Mulder, W. J. M.; Strijkers, G. J.; van Tilborg, G. A. F.; Griffioen, A. W.; Nicolay, K. NMR Biomed. 2006, 19, 142.

(6) Nicolle, G. M.; Tóth, É.; Eisenwiener, K.-P.; Mäcke, H. R.; Merbach, A. E. J. Biol. Inorg. Chem. 2002, 7, 757. 
(7) Mulder, W. J. M.; Strijkers, G. J.; van Tilborg, G. A. F.; Cormode, D. P.; Fayad, Z. A.; Nicolay, K. Acc. Chem. Res. 2009, 42, 904.

(8) Werner, E. J.; Datta, A.; Jocher, C. J.; Raymond, K. N. Angew. Chem., Int. Ed. 2008, 47, 8568.

(9) Allen, M.; Bulte, J. W. M.; Liepold, L.; Basu, G.; Zywicke, H. A.; Frank, J. A.; Young, M.; Douglas, T. Magn. Reson. Med. 2005, 54, 807.

(10) Datta, A.; Hooker, J. M.; Botta, M.; Francis, M. B.; Aime, S.; Raymond, K. N. J. Am. Chem. Soc. 2008, 130, 2546.

(11) Garimella, P. D.; Datta, A.; Romanini, D. W.; Raymond, K. N.; Francis, M. B. J. Am. Chem. Soc. 2011, 133, 14704.

(12) Hooker, J. M.; Datta, A.; Botta, M.; Raymond, K. N.; Francis, M. B. Nano Lett. 2007, 7, 2207.

(13) Liepold, L.; Anderson, S.; Willits, D.; Oltrogge, L.; Frank, J. A.; Douglas, T.; Young, M. Magn. Reson. Med. 2007, 58, 871.

(14) Liepold, L. O.; Abedin, M. J.; Buckhouse, E. D.; Frank, J. A.; Young, M. J.; Douglas, T. Nano Lett. 2009, 9, 4520.

(15) Prasuhn, J. D. E.; Yeh, R. M.; Obenaus, A.; Manchester, M.; Finn, M. G. Chem. Comm. 2007, 1269.

(16) Anderson, E. A.; Isaacman, S.; Peabody, D. S.; Wang, E. Y.; Canary, J. W.; Kirshenbaum, K. Nano Lett. 2006, 6, 1160.

(17) Pokorski, J. K.; Breitenkamp, K.; Liepold, L. O.; Qazi, S.; Finn, M. G. J. Am. Chem. Soc. 2011, 133, 9242.

(18) Brasch, M.; de la Escosura, A. s.; Ma, Y.; Uetrecht, C.; Heck, A. J. R.; Torres, T.; Cornelissen, J. J. L. M. J. Am. Chem. Soc. 2011, 133, 6878.

(19) de la Escosura, A.; Janssen, P. G. A.; Schenning, A. P. H. J.; Nolte, R. J. M.; Cornelissen, J. J. L. M. Angew. Chem., Int. Ed. 2010, 49, 5335.

(20) Minten, I. J.; Ma, Y.; Hempenius, M. A.; Vancso, G. J.; Nolte, R. J. M.; Cornelissen, J. J. L. M. Org. Biomol. Chem. 2009, 7, 4685.

(21) Sikkema, F. D.; Comellas-Aragones, M.; Fokkink, R. G.; Verduin, B. J. M.; Cornelissen, J. J. L. M.; Nolte, R. J. M. Org. Biomol. Chem. 2007, 5, 54.

(22) Kwak, M.; Minten, I. J.; Anaya, D.-M.; Musser, A. J.; Brasch, M.; Nolte, R. J. M.; Müllen, K.; Cornelissen, J. J. L. M.; Herrmann, A. J. Am. Chem. Soc. 2010, 132, 7834.

(23) Telgmann, L.; Holtkamp, M.; Kunnemeyer, J.; Gelhard, C.; Hartmann, M.; Klose, A.; Sperling, M.; Karst, U. Metallomics 2011, 3, 1035.

(24) Reddy, V. S.; Natarajan, P.; Okerberg, B.; Li, K.; Damodaran, K. V.; Morton, R. T.; Brooks, C. L.; Johnson, J. E. J. Virol. 2001, 75, 11943.

(25) Speir, J. A.; Munshi, S.; Wang, G.; Baker, T. S.; Johnson, J. E. Structure 1995, 3, 63.

(26) Cadena-Nava, R. D.; Hu, Y.; Garmann, R. F.; Ng, B.; Zelikin, A. N.; Knobler, C. M.; Gelbart, W. M. J. Phys. Chem. B 2011, 115, 2386.

(27) Aniagyei, S. E.; Kennedy, C. J.; Stein, B.; Willits, D. A.; Douglas, T.; Young, M. J.; De, M.; Rotello, V. M.; Srisathiyanarayanan, D.; Kao, C. C.; Dragnea, B. Nano Lett. 2009, 9, 393.

(28) Aniansson, E. A. G.; Wall, S. N.; Almgren, M.; Hoffmann, H.; Kielmann, I.; Ulbricht, W.; Zana, R.; Lang, J.; Tondre, C. J. Phys. Chem. 1976, 80, 905.

(29) Patist, A.; Oh, S. G.; Leung, R.; Shah, D. O. Colloids Surf., A 2001, 176, 3. 
(30) Ferreira, M. F.; Mousavi, B.; Ferreira, P. M.; Martins, C. I. O.; Helm, L.; Martins, J. A.; Geraldes, C. F. G. C. Dalton Trans. 2012, 41, 5472.

(31) Nicolle, G. M.; Helm, L.; Merbach, A. E. Magn. Reson. Chem. 2003, 41, 794.

(32) Jori, G.; Coppellotti, O. Anti-Infect. Agents Med. Chem. 2007, 6, 119.

(33) O’Connor, A. E.; Gallagher, W. M.; Byrne, A. T. Photochem. Photobiol. 2009, 85, 1053.

(34) Dhaon, M. K.; Olsen, R. K.; Ramasamy, K. J. Org. Chem. 1982, 47, 1962.

(35) Beeby, A.; Bushby, L. M.; Maffeo, D.; Gareth Williams, J. A. J. Chem. Soc., Dalton Trans. 2002, 48.

(36) Vetterlein, K.; Bergmann, U.; Büche, K.; Walker, M.; Lehmann, J.; Linscheid, M. W.; Scriba, G. K. E.; Hildebrand, M. Electrophoresis 2007, 28, 3088.

(37) Comellas-Aragones, M.; Engelkamp, H.; Claessen, V. I.; Sommerdijk, N. A. J. M.; Rowan, A. E.; Christianen, P. C. M.; Maan, J. C.; Verduin, B. J. M.; Cornelissen, J. J. L. M.; Nolte, R. J. M. Nat. Nanotechnol. 2007, 2, 635.

(38) Verduin, B. J. M. FEBS Lett. 1974, 45, 50.

(39) Corsi, D. M.; Platas-Iglesias, C.; Bekkum, H. v.; Peters, J. A. Magn. Reson. Chem. 2001, 39, 723.

(40) Peters, J. A.; Huskens, J.; Raber, D. J. Prog. Nucl. Magn. Reson. Spectrosc. 1996, 283. 


\section{Appendix 4.1. Encapsulation of ZnPc Dye}

In order to study the behavior (in aqueous solution) of the $\mathrm{ZnPc}$ dye, absorption spectra in the absence and presence of ligand 5 were measured. This was done by adding (drop wise) $50 \mu \mathrm{L}$ of a $5.5 \times 10^{-6} \mathrm{M}$ $\mathrm{ZnPc}$ solution in $\mathrm{MeOH}$ to $1 \mathrm{~mL}$ of water and to a $0.04 \mathrm{M} 1 \mathrm{~mL}$ solution of 5. After addition of the dye, both solutions were heated for 2 minutes, allowed to cool down and refilled to $1 \mathrm{~mL}$ with water. While a (blue) precipitate was observed in the solution containing only water, the solution containing 5 remained colored (blue) after treatment. This last observation indicates the encapsulation of one $\mathrm{ZnPc}$ dye per DOTAC10 micelle, as the optical properties of the $\mathrm{ZnPc}$ dye are affected when forming $\pi$-stacked aggregates in water. Spectroscopic evidence for this observation is shown in Figure 4.8; in the presence of ligand 5 (black line) a strong absorption peak at around $682 \mathrm{~nm}$ appears, while in the absence of $\mathbf{5}$ (grey line) very weak absorption is observed.

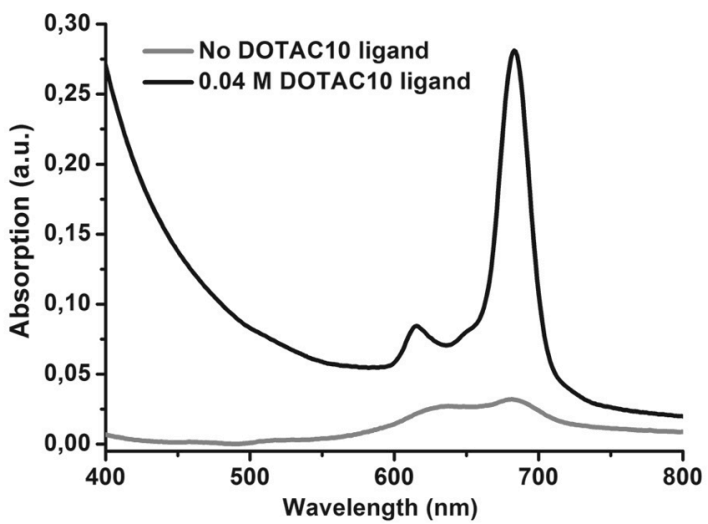

Figure 4.8. UV absorption spectrum of $\mathrm{ZnPc}$ before (grey line) and after (black line) encapsulation in a $0.04 \mathrm{M}$ aqueous solution of ligand $\mathbf{5}$. 


\section{Appendix 4.2. TXRF Data, Capsid Concentration and Fncapsulation Ffficiency Calculations}

Table 4.1. Capsid, $\mathrm{Zn}^{2+}$ and $\mathrm{Gd}^{3+}$ concentrations for all samples

\begin{tabular}{|c|c|c|c|c|}
\hline $\begin{array}{l}\text { Protein cage filled } \\
\text { with micelles of }\end{array}$ & $\begin{array}{c}\text { [capsid] } \\
\text { (nM) }\end{array}$ & $\begin{array}{l}{\left[\mathrm{Zn}^{2+}\right] \mathrm{mg} / \mathrm{L} /} \\
{\left[\mathrm{Gd}^{3+}\right] \mathrm{mg} / \mathrm{L}^{*}}\end{array}$ & $\begin{array}{c}\text { \# } \mathrm{Zn}^{2+} \text { atoms per } \\
\text { capsid/ }\left[\mathrm{Zn}^{2+}\right] \\
\mathrm{mM}\end{array}$ & $\begin{array}{c}\text { \# } \mathrm{Gd}^{3+} \text { atoms } \\
\text { per capsid / } \\
{\left[\mathrm{Gd}^{3+}\right] \mathrm{mM}}\end{array}$ \\
\hline $\mathrm{Gd}^{3+}$-DOTAC10 & 364 & $---/ 0.167$ & --- & $3 / 0.001062$ \\
\hline $\mathrm{ZnPc}$ & 125 & $\begin{array}{l}0.075 \text { (corrected } \\
\text { value } 0.026) /---\end{array}$ & $3 / 0.0003975$ & --- \\
\hline $\begin{array}{c}\mathrm{Gd}^{3+}-\mathrm{DOTAC} 10 / \mathrm{ZnPc} \\
\text { (non-diluted) }\end{array}$ & 1022 & $\begin{array}{c}1.048 \text { (corrected } \\
\text { value } \\
0.99) / 56.471\end{array}$ & $15 / 0.015$ & $350 / 0.359$ \\
\hline $\begin{array}{c}\mathrm{Gd}^{3+}- \\
\text { DOTAC } 10 / \mathrm{ZnPc}{ }^{\star *}\end{array}$ & 128 & $0.124 / 7.059$ & $15 / 0.0019$ & $350 / 0.0449$ \\
\hline
\end{tabular}

* Values as shown in Tables S2, S3 and S4. As $\mathrm{Zn}^{2+}$ was detected in capsid buffer, these values are corrected

${ }^{\star \star}$ This sample was diluted 8 times (after elemental analysis) for MRI/relaxivity measurements

Table 4.2. TXRF analysis data of the capsid filled with $\mathrm{Gd}^{3+}$-DOTAC10 micelles

\begin{tabular}{|c|c|c|c|c|c|c|c|c|}
\hline Element & Line & $\begin{array}{c}\text { Energy } \\
(\mathbf{k e V})\end{array}$ & Net & Backgr & $\sigma$ & $\begin{array}{c}{[\mathbf{C}]} \\
(\mathbf{m g} / \mathbf{l})\end{array}$ & $\begin{array}{c}\sigma[\mathbf{C}] \\
(\mathbf{m g} / \mathbf{l})\end{array}$ & $\begin{array}{c}\text { LLD } \\
(\mathbf{m g} / \mathbf{l})\end{array}$ \\
$\mathrm{Cl}$ & $\mathrm{K} 12$ & 2.622 & 19620461 & 134674 & 4460 & 1100354 & 10.624 & 0.617 \\
$\mathrm{~K}$ & $\mathrm{~K} 12$ & 3.314 & 1232 & 97895 & 444 & 0.29 & 0.105 & 0.221 \\
$\mathrm{Ca}$ & $\mathrm{K} 12$ & 3.692 & 18824 & 96328 & 460 & 3.182 & 0.08 & 0.157 \\
$\mathrm{Fe}$ & $\mathrm{K} 12$ & 6.405 & 9400 & 94935 & 446 & 0.35 & 0.017 & 0.034 \\
$\mathrm{Ga}$ & $\mathrm{K} 12$ & 9.251 & 585008 & 95556 & 881 & 10 & 0.022 & 0.016 \\
$\mathrm{Ga}$ & $\mathrm{L} 1$ & 1.098 & 73072 & 203406 & 693 & --- & --- & --- \\
$\mathrm{Br}$ & $\mathrm{K} 12$ & 11.924 & 262532 & 130632 & 724 & 3.221 & 0.011 & 0.013 \\
$\mathrm{Br}$ & $\mathrm{L} 1$ & 1.481 & 10911 & 192045 & 628 & --- & --- & -- \\
$\mathrm{Gd}$ & $\mathrm{L} 1$ & 6.053 & 2815 & 91840 & 432 & 0.167 & 0.026 & 0.054 \\
\hline
\end{tabular}


Table 4.3. TXRF analysis data of the capsid filled with DOTAC10/ZnPc micelles

\begin{tabular}{|c|c|c|c|c|c|c|c|c|}
\hline Element & Line & $\begin{array}{c}\text { Energy } \\
(\mathbf{k e V})\end{array}$ & Net & Backgr & $\sigma$ & $\begin{array}{c}{[\mathbf{C}]} \\
(\mathbf{m g} / \mathbf{l})\end{array}$ & $\begin{array}{c}\sigma[\mathbf{C}] \\
(\mathbf{m g} / \mathbf{l})\end{array}$ & $\begin{array}{c}\text { LLD } \\
(\mathbf{m g} / \mathbf{l})\end{array}$ \\
\hline $\mathrm{Cl}$ & $\mathrm{K} 12$ & 2.622 & 10574625 & 57564 & 3270 & 11742.05 & 14.471 & 0.799 \\
$\mathrm{~K}$ & $\mathrm{~K} 12$ & 3.314 & 2843 & 39852 & 287 & 1.326 & 0.135 & 0.279 \\
$\mathrm{Ca}$ & $\mathrm{K} 12$ & 3.692 & 4061 & 38274 & 284 & 1.359 & 0.096 & 0.196 \\
$\mathrm{Fe}$ & $\mathrm{K} 12$ & 6.405 & 2219 & 38538 & 282 & 0.163 & 0.021 & 0.043 \\
$\mathrm{Zn}$ & $\mathrm{K} 12$ & 8.637 & 1922 & 38133 & 280 & 0.075 & 0.011 & 0.023 \\
$\mathrm{Ga}$ & $\mathrm{K} 12$ & 9.251 & 295465 & 39068 & 611 & 10 & 0.03 & 0.02 \\
$\mathrm{Ga}$ & $\mathrm{L} 1$ & 1.098 & 28078 & 89470 & 455 & --- & --- & -- \\
$\mathrm{Br}$ & $\mathrm{K} 12$ & 11.924 & 46203 & 51933 & 387 & 1.122 & 0.01 & 0.017 \\
$\mathrm{Br}$ & $\mathrm{L} 1$ & 1.481 & 1 & 84976 & 412 & --- & --- & --- \\
\hline
\end{tabular}

Table 4.4. TXRF analysis data of the capsid filled with $\mathrm{Gd}^{3+}$-DOTAC10/ZnPc micelles

\begin{tabular}{|c|c|c|c|c|c|c|c|c|}
\hline Element & Line & $\begin{array}{l}\text { Energy } \\
\text { (keV) }\end{array}$ & Net & Backgr & $\sigma$ & [C] (mg/l) & $\begin{array}{c}\sigma[\mathrm{C}] \\
(\mathrm{mg} / \mathrm{l})\end{array}$ & $\begin{array}{l}\text { LLD } \\
\text { (mg/l) }\end{array}$ \\
\hline $\mathrm{Cl}$ & K12 & 2.622 & 15265615 & 55336 & 3921 & 22817.842 & 73.901 & 1.055 \\
\hline $\mathrm{K}$ & K12 & 3.314 & 4536 & 35447 & 275 & 2.847 & 0.181 & 0.354 \\
\hline $\mathrm{Ca}$ & K12 & 3.692 & 9874 & 34884 & 282 & 4.449 & 0.14 & 0.252 \\
\hline $\mathrm{Fe}$ & K12 & 6.405 & 1884 & 36742 & 275 & 0.187 & 0.028 & 0.057 \\
\hline $\mathrm{Zn}$ & K12 & 8.637 & 20051 & 36377 & 305 & 1.048 & 0.019 & 0.03 \\
\hline $\mathrm{Ga}$ & K12 & 9.251 & 219495 & 35924 & 540 & 10 & 0.054 & 0.026 \\
\hline $\mathrm{Ga}$ & $\mathrm{L} 1$ & 1.098 & 32703 & 87820 & 456 & --- & --- & --- \\
\hline $\mathrm{Br}$ & K12 & 11.924 & 93549 & 45807 & 430 & 3.059 & 0.023 & 0.021 \\
\hline $\mathrm{Br}$ & L1 & 1.481 & 3628 & 84274 & 415 & --- & --- & --- \\
\hline Gd & L1 & 6.053 & 358050 & 35163 & 655 & 56.471 & 0.272 & 0.089 \\
\hline
\end{tabular}




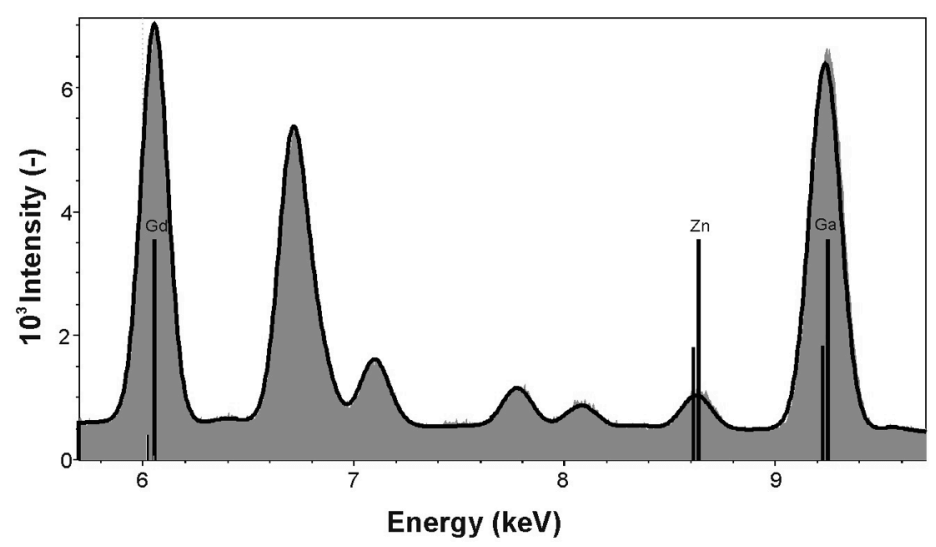

Figure 4.9. TXRF spectrum of the capsid filled with $\mathrm{Gd}^{3+}$-DOTAC10/ZnPc micelles.

\section{DOTAC10/ZnPc Encapsulation}

Calculations based on a $1 \mathrm{~mL}$ sample:

\# capsids $=1 \mathrm{~mL}$ solution $x \frac{125 \mathrm{nmol}}{\mathrm{L}} \times \frac{1 \mathrm{~mol}}{10^{9} \mathrm{nmol}} \times \frac{6.023 \times 10^{23} \mathrm{capsids}}{1 \mathrm{~mol}} \times \frac{1 \mathrm{~L}}{1000 \mathrm{~mL}}$

$$
=7.53 \times 10^{13}
$$

\# ZnPc dyes

$=1 \mathrm{~mL}$ solution $x \frac{0.0003975 \mathrm{mmol} \mathrm{Zn}{ }^{2+}}{L} \times \frac{1 \mathrm{~mol}}{1000 \mathrm{mmol}} \times \frac{6.023 \times 10^{23} \mathrm{atoms} \mathrm{Zn}^{2+}}{1 \mathrm{~mol}} \times \frac{1 \mathrm{~L}}{1000 \mathrm{~mL}}$

$=2.39 \times 10^{14}$

Assuming that 1 dye is encapsulated by one micelle:

$\#$ micelles $=\#$ ZnPc dyes $=2.39 \times 10^{14}$

$\# Z n^{2+}$ per capsid $=\frac{2.39 \times 10^{14} \mathrm{Zn}^{2+} \text { dyes }}{7.53 \times 10^{13} \text { capsids }}=3 Z^{2+}$ dyes capsid 
Based on these numbers, on average, three micelles are present in one $(T=3)$ capsid.

\section{$G^{3+}$-DOTAC10/ZnPc Encapsulation}

Calculations based on a $1 \mathrm{~mL}$ sample:

$$
\begin{aligned}
& \text { \# monomers } \\
& =1 \mathrm{~mL} \text { solution } x \frac{0.0449 \mathrm{mmol} \mathrm{Gd} d^{3+}}{L} \times \frac{1 \mathrm{~mol}}{1000 \mathrm{mmol}} \times \frac{6.023 \times 10^{23}{\mathrm{atoms} G d^{3+}}_{1 \mathrm{~mol}}}{1000 \mathrm{~mL}} \frac{1 \mathrm{~L}}{1000} \\
& =2.7 \times 10^{16}
\end{aligned}
$$

\# ZnPc dyes

$=1 \mathrm{~mL}$ solution $x \frac{0.00191 \mathrm{mmol} \mathrm{Zn}^{2+}}{\mathrm{L}} \times \frac{1 \mathrm{~mol}}{1000 \mathrm{mmol}} \times \frac{6.023 \times 10^{23} \mathrm{atoms}^{2+}}{1 \mathrm{~mol}} \times \frac{1 \mathrm{~L}}{1000 \mathrm{~mL}}$ $=1.15 \times 10^{15}$

$\#$ micelles $=2.7 \times 10^{16}$ monomers $x \frac{1 \text { micelle }}{15 \text { monomers }}=1.8 \times 10^{15}$

\# capsids $=1 \mathrm{~mL}$ solution $x \frac{128 \mathrm{nmol}}{\mathrm{L}} \times \frac{1 \mathrm{~mol}}{10^{9} \mathrm{nmol}} \times \frac{6.023 \times 10^{23} \mathrm{capsids}}{1 \mathrm{~mol}} \times \frac{1 \mathrm{~L}}{1000 \mathrm{~mL}}$

$$
=7.71 \times 10^{13}
$$

$\# Z n^{2+}$ per capsid $=\frac{1.15 \times 10^{15} \mathrm{Zn}^{2+} \text { dyes }}{7.71 \times 10^{13} \text { capsids }}=15 \mathrm{Zn}^{2+}$ dyes capsid

$\# G d^{3+}$ per capsid $=\frac{2.7 \times 10^{16} G d^{3+} \text { ions or monomers }}{7.71 \times 10^{13} \text { capsids }}=350 G d^{3+}$ per capsid

$\#$ micelles per capsid $=\frac{1.8 \times 10^{15} \text { micelles }}{7.71 \times 10^{13} \text { capsids }}=23$

Based on these numbers, on average, a maximum of $\mathbf{2 3}$ micelles are present in one $(T=3)$ capsid. 


\section{Appendix 4.3. TFM Analysis}

For the sake of comparison, the size distribution of the capsid filled with only the ligand (5) was assessed (Figure 4.10), showing a mean internal diameter of $24.8 \mathrm{~nm}$. Mean sizes of each capsid are summarized in Table 4.5 .

Table 4.5. Average size of the different protein cages

\begin{tabular}{|c|c|c|c|c|}
\hline $\begin{array}{c}\text { Protein cage filled with } \\
\text { micelles of }\end{array}$ & DOTAC10 & $\begin{array}{c}\mathrm{Gd}^{3+}- \\
\text { DOTAC10 }\end{array}$ & DOTAC10/ZnPc & $\begin{array}{c}\mathrm{Gd}^{3+}- \\
\text { DOTAC10/ZnPc }\end{array}$ \\
\hline Mean diameter $/ \mathrm{nm}$ & 24.8 & 24.6 & 24.3 & 22.4 \\
\hline Standard deviation / & 3 & 3 & 3 & 2 \\
\hline
\end{tabular}

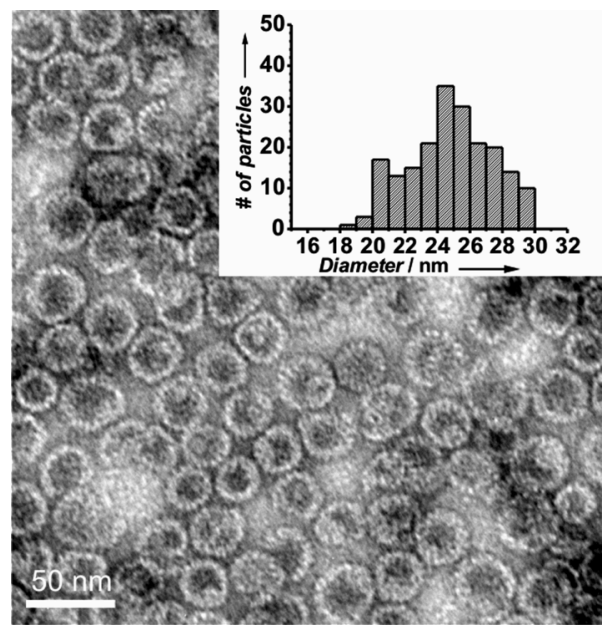

Figure 4.10. TE micrograph of protein cages filled with only micelles of DOTAC10 (5). Inset: particle size distribution histogram showing that the mean internal diameter is $24.8 \mathrm{~nm}$. Scale bar: $50 \mathrm{~nm}$. 


\section{Appendix 4.4. MRI Data}

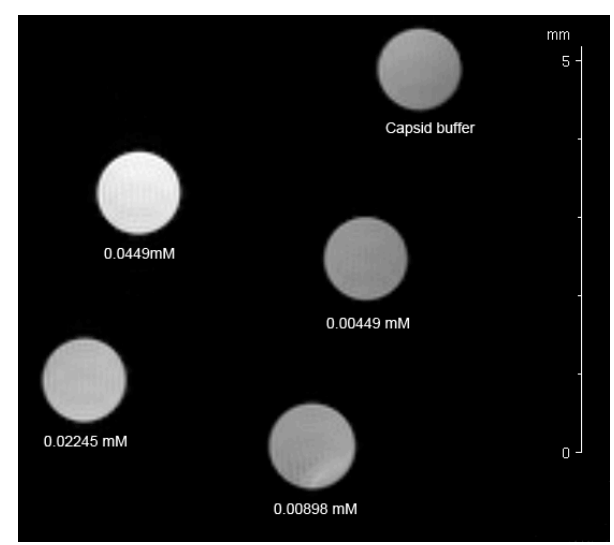

Figure 4.11. $T_{1}$-weighted image of solutions of different concentrations of capsid filled with $\mathrm{Gd}^{3+}$-DOTAC10/ZnPc micelles (shown as $\mathrm{Gd}^{3+}$ concentration) at $600 \mathrm{MHz}$ and $25^{\circ} \mathrm{C}$. Slice thickness $6 \mathrm{~mm}, 10$ averages, matrix size: $256 \times 256$, TE $=6 \mathrm{~ms}$ and TR $=500 \mathrm{~ms}$

Table 4.6. $T_{1}$ values obtained for the capsid filled with $\mathrm{Gd}^{3+}$-DOTAC10/ZnPc micelles at $600 \mathrm{MHz}$ and $25^{\circ} \mathrm{C}$

\begin{tabular}{|c|c|c|c|c|c|c|c|c|}
\hline $\begin{array}{c}\text { Gd }^{3+} \text { ] } \\
\mathbf{m M}\end{array}$ & $\begin{array}{c}\boldsymbol{T}_{\mathbf{1}} \text { Slice } \\
\mathbf{1}(\mathbf{m s})\end{array}$ & $\begin{array}{c}\boldsymbol{T}_{1} \text { Slice } \\
\mathbf{2}(\mathbf{m s})\end{array}$ & $\begin{array}{c}\boldsymbol{T}_{\mathbf{1}} \text { Slice } \\
\mathbf{3}(\mathbf{m s})\end{array}$ & $\begin{array}{c}\boldsymbol{T}_{\mathbf{1}} \text { Slice } \\
\mathbf{4}(\mathbf{m s})\end{array}$ & $\begin{array}{c}\boldsymbol{T}_{\mathbf{1}} \text { Slice } \\
\mathbf{5}(\mathbf{m s})\end{array}$ & $\begin{array}{c}\text { Average } \\
(\mathbf{m s})\end{array}$ & $\begin{array}{c}\text { Standard } \\
\text { Deviation } \\
(\mathbf{m s})\end{array}$ & $\begin{array}{c}\mathbf{1 / T _ { 1 }} \\
\left(\mathbf{s}^{-1}\right)\end{array}$ \\
\hline 0.0449 & 1946.8 & 2015.6 & 2037.0 & 2019.5 & 2019.5 & 2007.7 & 35.0 & 0.498 \\
\hline 0.02245 & 2294.1 & 2386.7 & 2360.4 & 2385.7 & 2354.0 & 2356.2 & 37.7 & 0.424 \\
\hline 0.00898 & 2796.3 & 2835.3 & 2807.5 & 2799.9 & 2755.4 & 2798.9 & 28.7 & 0.357 \\
\hline 0.00449 & 2948.2 & 2954.7 & 2878.0 & 2864.4 & 2795.6 & 2888.2 & 65.7 & 0.346 \\
\hline 0 & 3125.8 & 3243.8 & 3218.4 & 3301.2 & 3279.2 & 3233.7 & 68.2 & 0.309 \\
\hline
\end{tabular}


CHAPTER 4 
"The only difference between the Sane and the Insane, is IN and yet within this world, the Sane have the power to have the Insane locked up" Hunther S. Thompson

\section{CHAPTER 5}

\section{MULTIMODAL GOLD NANORODS FOR MAGNETIC RESONANCE AND FLUORESCENCE IMAGING}

\section{Abstract}

In this chapter, the synthesis, characterization and study of the cytotoxicity of multimodal gold nanorods (MMAuNRs) for magnetic resonance and optical imaging is described. The surface of these nanostructures was modified by means of thiol-based ligand exchange and the resulting AuNRs were characterized by means of UV-Vis absorption spectroscopy, transmission electron microscopy (TEM) and total reflection $x$-ray fluorescence (TXRF). Using various incubation percentages of O-[2-(3-mercaptopropionylamino)ethyl]-O'-methylpolyethylene glycol (PEG-SH) and $\mathrm{Gd}^{3+}-1-(11-$ sulfanylundecyl)-4,7,10-tris(carboxymethyl)1,4,7,10-tetraazacyclododecane ( $\mathrm{Gd}^{3+}$-SUDDO3A) a good compromise between biocompatibility/colloidal stability and $\mathrm{Gd}^{3+}$ loading is attainable. The second imaging modality was added by incorporating (3aS,4S,6aR)-11-sulfanylundecyl 5(hexahydro-2-oxo-1H-thieno[3,4-d]imidazol-4-yl) pentanoate (Biotin-SH) during the ligand exchange process. Subsequent incubation with Cy5-streptavidin led to paramagnetic and fluorescent AuNRs, visualized by $\mathrm{MRI}$ and fluorescence microscopy. The MMAuNRs obtained using this one-pot synthesis are biocompatible, show median lethal concentration $\left(\mathrm{LC}_{50}\right)$ values > $150 \mathrm{pM}$ AuNR, improved relaxivity properties, high $\mathrm{Gd}^{3+}$ loadings and can be optically colocalized with mouse myoblast (C2C12) cells in vitro. 


\subsection{Introduction}

Magnetic resonance imaging (MRI) is a well-established technique used in medical diagnostics due to its non-ionizing character and the excellent soft tissue contrast and spatial resolution it offers. ${ }^{1,2}$ This technique takes advantage of the different longitudinal $\left(T_{1}\right)$ relaxation times most body tissues have. However, when different tissues show similar relaxation behavior, a contrast agent (CA, typically a paramagnetic $\mathrm{Gd}^{3+}$ complex) $)^{3,4}$ is used to aid the image contrast by shortening $T_{1}$. The $T_{1}$-shortening efficiency shown by a CA is known as the relaxivity $r_{1}$.

Due to the inherent lack of sensitivity of MRI and the large amount of water found in the human body, high concentrations of CAs are usually required. In the past, CAs with enhanced paramagnetic properties were developed by: i) increasing the "local" $\mathrm{Gd}^{3+}$ concentration and ii) decreasing the global rotational motion (the tumbling rate $\tau_{R}$ ) of the $\mathrm{Gd}^{3+}$ complexes used. One of the most popular strategies followed is the surface modification of inorganic spherical nanostructures such as fluorescent quantum dots ${ }^{5-7}$ and gold nanoparticles ${ }^{8-13}$ with functionalized $\mathrm{Gd}^{3+}$ chelates. These nanoparticle-based systems ${ }^{16,17}$ not only show enhanced relaxivity properties but have also been used for other imaging modalities such as optical imaging ${ }^{5-7,12}$ or X-ray computed tomography (CT). ${ }^{8}$

Gold nanoparticles are appealing for biological applications due to their light absorption and scattering properties derived from localized surface plasmon resonance (LSPR), often observed as strong extinction peaks in the visible and near-infrared regions. ${ }^{14}$ Fine-tuning of the optical properties of these nanostructures can be achieved by altering their size and morphology. For example, gold nanorods (AuNRs) ${ }^{15,16}$ show two prominent SPR peaks originating from the excitation of surface plasmon oscillations 
along both the short axis (transverse band, located around $520 \mathrm{~nm}$ ) and the long axis (longitudinal band, located in the near-infrared region). The near-infrared absorption band, whose position strongly depends on the aspect ratio (length/width) of the AuNRs, favors their use for biomedical applications, ${ }^{17-19}$ e.g, as photothermal therapy agents ${ }^{20,21}$ or for photoacoustic imaging. ${ }^{22}$

More recently, AuNR-based CAs for MRI have been synthesized by means of ligand exchange and subsequent covalent ${ }^{23}$ and non-covalent ${ }^{24}$ functionalization with paramagnetic ligands. In both cases, CAs with enhanced relaxivity properties were obtained. While these examples nicely illustrate the potential of AuNRs as multimodal imaging agents (IAs) by combining MRI with $\mathrm{CT}^{24}$ and plasmonic imaging, ${ }^{23}$ the integration of a more widespread second modality as optical imaging has not been investigated yet. In this chapter, the use of AuNRs as platforms for the one-pot synthesis of multimodal IAs is proposed. Herein, the facile synthesis of biocompatible, multimodal AuNRs (MMAuNRs) suitable for magnetic resonance and optical imaging is described. The elongated structure of the AuNRs, available for ligand exchange, makes them excellent platforms for the integration of paramagnetic, fluorescent and biocompatible moieties. Consequently, MMAuNRs represent a novel class of IAs with expected improved relaxivity properties due to high $\mathrm{Gd}^{3+}$ payloads per particle and interesting optical properties due to the incorporation of fluorescent dyes.

\subsection{Design and Synthesis}

Whereas one of the major advantages of AuNRs is their straightforward and well-described synthesis, large quantities of cetyl trimethylammonium 
bromide (CTAB, which is known to be toxic) ${ }^{25,26}$ are required. However, surface modification of AuNRs allows the incorporation of biocompatible ligands, ${ }^{27,28}$ as well as biomarkers (targeting), ${ }^{20}$ ligands for enhanced cellular uptake $\mathrm{e}^{29}$ and quantum $\operatorname{dots}^{30}$ and organic dyes for optical imaging purposes. $^{31}$

Here, the gold nanostructures are coated, by simultaneous thiol-based ligand exchange, with an optimized combination of the $\mathrm{Gd}^{3+}$ complex of 1-(11-sulfanylundecyl)-4,7,10-tris(carboxymethyl)-1,4,7,10-

tetraazacyclododecane (6, $\left.\mathrm{Gd}^{3+}-\mathrm{SUDDO} 3 \mathrm{~A}\right)$ for $\mathrm{MRI}$ and O-[2-(3mercaptopropionylamino)ethyl]-O'-methylpolyethylene glycol (PEG-SH, $M_{w}=5000$ ) in order to increase their biocompatibility and solubility. Furthermore, (3aS,4S,6aR)-11-sulfanylundecyl 5-(hexahydro-2-oxo-1Hthieno[3,4-d]imidazol-4-yl) pentanoate (8, Biotin-SH) is used to incorporate (in a later stage) a fluorescent dye (Cy5) for optical imaging by means of the biotin-streptavidin interaction (Figure 5.1). ${ }^{32}$

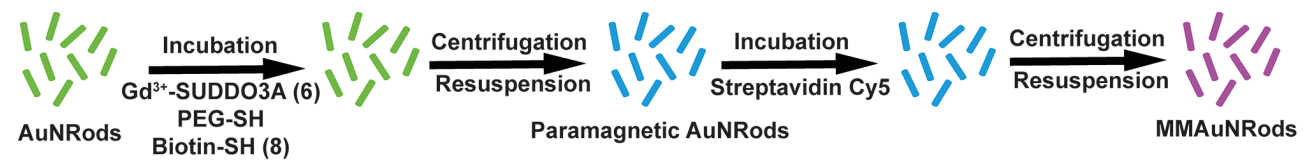

Figure 5.1. Schematic representation of the synthesis of MMAuNRs

\subsubsection{Synthesis of $\mathrm{Gd}^{3+-}$ SUDDO3A}

To synthesize the thiolated $\mathrm{Gd}^{3+}$ complex 1-(11-sulfanylundecyl)-4,7,10tris(carboxymethyl)-1,4,7,10-tetraazacyclododecane $\quad\left(\mathrm{Gd}^{3+}\right.$-SUDDO3A, Scheme 5.1), bromo-1-undecanthiol (1) was protected by reacting it with dihydropyran and pyridinium $p$-toluene sulfonate in $\mathrm{CH}_{2} \mathrm{Cl}_{2}$ at room temperature overnight. Compound (4) was then synthesized by alkylating 1,4,7-tris(tert-butoxycarboxymethyl)-1,4,7,10-tetraazacyclododecane 
with the protected thiol 11-bromo-1-undecyltetrahydropyranyl sulfide (2) in dry acetonitrile using $\mathrm{K}_{2} \mathrm{CO}_{3}$ (under $\mathrm{N}_{2}$ ) for three days at $50^{\circ} \mathrm{C}$. The SUDDO3A ligand (5) was obtained by means of Boc-deprotection of (4) using TFA $/ \mathrm{CH}_{2} \mathrm{Cl}_{2}$ at room temperature overnight. Lastly, the $\mathrm{Gd}^{3+}$ complex of SUDDO3A (6) was prepared by adding one equivalent of $\mathrm{GdCl}_{3}$ to a solution of (5) in water, adjusting the $\mathrm{pH}$ to 6 and stirring at $60^{\circ} \mathrm{C}$ for 1 day. The final product 6 was obtained with an overall yield (after purification) of $25 \%$.
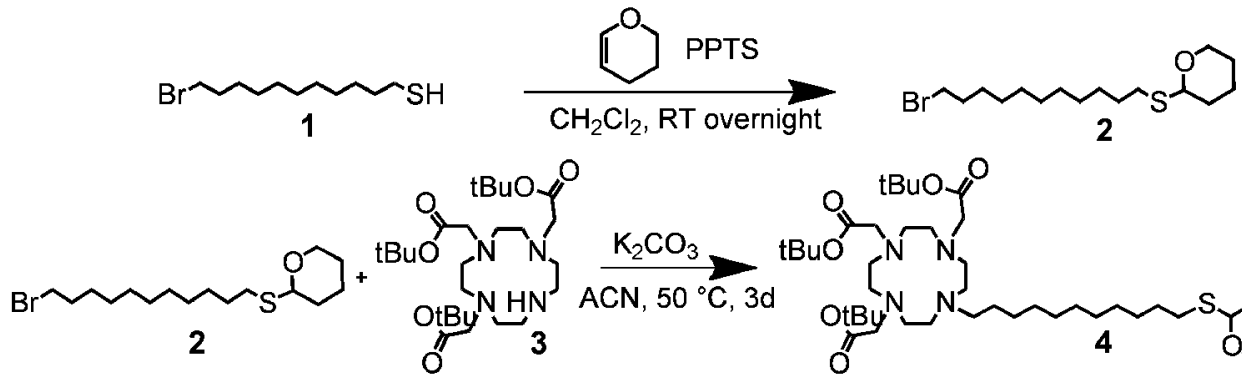

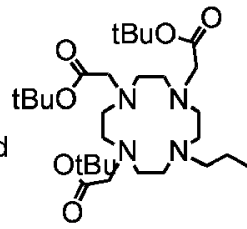
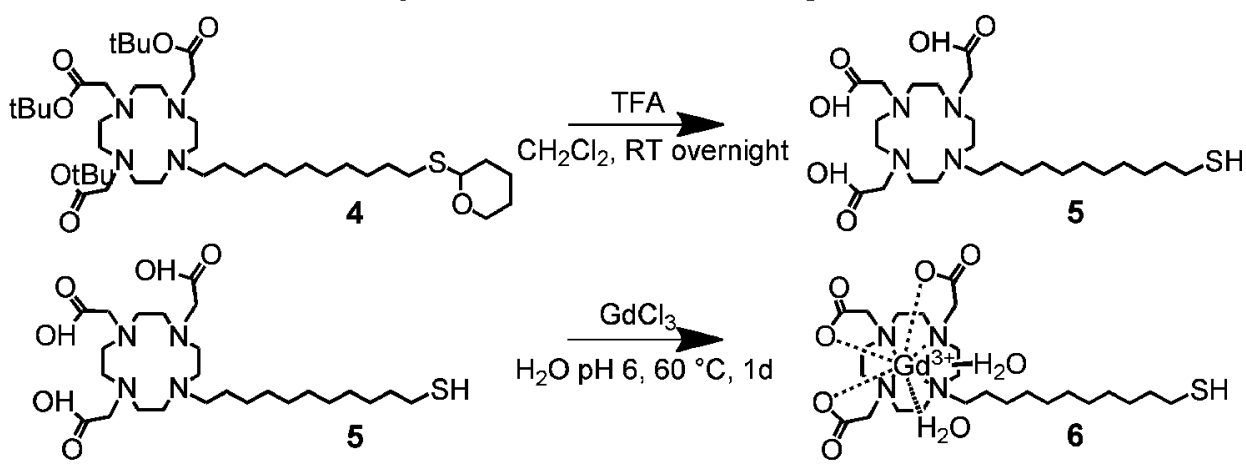

Scheme 5.1. Synthetic route followed for the synthesis of the $\mathrm{Gd}^{3+}$ complex of 1-(11sulfanylundecyl)-4,7,10-tris(carboxymethyl)-1,4,7,10-tetraazacyclododecane $\left(\mathrm{Gd}^{3+}-\right.$ SUDDO3A, 6).

\subsubsection{Synthesis of Biotin-SH}

The syntheses of (3aS,4S,6aR)-11-sulfanylundecyl 5-(hexahydro-2-oxo$1 \mathrm{H}$-thieno[3,4-d]imidazol-4-yl) pentanoate (biotin-SH) and its brominated 
precursor (Scheme 5.2, 7 and 8, respectively) were carried out following a literature procedure (see experimental section). ${ }^{33}$ The final product 8 was obtained with an overall yield of $60 \%$.
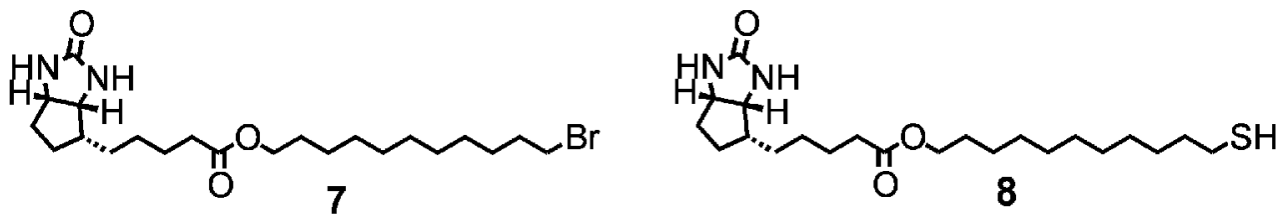

Scheme 5.2. Structures of (3aS,4S,6aR)-11-sulfanylundecyl 5-(hexahydro-2-oxo-1Hthieno[3,4-d]imidazol-4-yl) pentanoate (biotin-SH, 8) and the brominated precursor 7

\subsubsection{AuNRs Synthesis and Characterization}

The AuNRs were grown following a previously described seed-mediated growth procedure. ${ }^{34}$ All glassware was cleaned with a 3:1 solution of concentrated $\mathrm{H}_{2} \mathrm{SO}_{4}$ and $30 \% \mathrm{H}_{2} \mathrm{O}_{2}$ prior to use, rinsed abundantly with Milli-Q water and dried in the oven overnight $\left(60^{\circ} \mathrm{C}\right)$. The gold seeds were prepared by placing a (water) solution consisting of $5 \mathrm{~mL}$ of $0.0005 \mathrm{M}$ $\mathrm{HAuCl}_{4}$ and $5 \mathrm{~mL}$ of $0.2 \mathrm{M} \mathrm{CTAB}$ in an oil bath at $27^{\circ} \mathrm{C}$, stirring at 1000 rpm. Rapid addition of a solution of $600 \mu \mathrm{L}$ ice cold $\mathrm{NaBH}_{4}(0.01 \mathrm{M})$ led to the formation of seed particles. This seed solution was stirred for exactly 2 min and allowed to stand for another $10 \mathrm{~min}$ in an oil bath at $27^{\circ} \mathrm{C}$. Meanwhile, the growth solution was prepared by mixing in the following order $25 \mu \mathrm{L}$ freshly prepared $0.04 \mathrm{M} \mathrm{AgNO}_{3}, 5 \mathrm{~mL} 0.2 \mathrm{M} \mathrm{CTAB}, 20 \mu \mathrm{L}$ $0.5 \mathrm{M} \mathrm{H}_{2} \mathrm{SO}_{4}, 5 \mathrm{~mL} 0.001 \mathrm{M} \mathrm{HAuCl}_{4}$ and $70 \mu \mathrm{L}$ freshly prepared $0.0788 \mathrm{M}$ ascorbic acid. The solution was gently mixed immediately until the color changed from dark yellow to colorless. Finally, $12 \mu \mathrm{L}$ of the seed solution were slowly added to the growth solution in an oil bath at $27^{\circ} \mathrm{C}$. The final solution was incubated for at least 2 hours and analyzed without further purification. The synthesized AuNRs were characterized by UV-Vis 
absorption spectroscopy and transmission electron microscopy (TEM). Results revealed that the two synthesized batches, Batch \#1 and Batch \#2, show longitudinal plasmon band maxima at 744 and $771 \mathrm{~nm}$ (Figure 5.2 , left) and aspect ratios of 3.5 and 3.8 respectively (Figure 5.2, right).
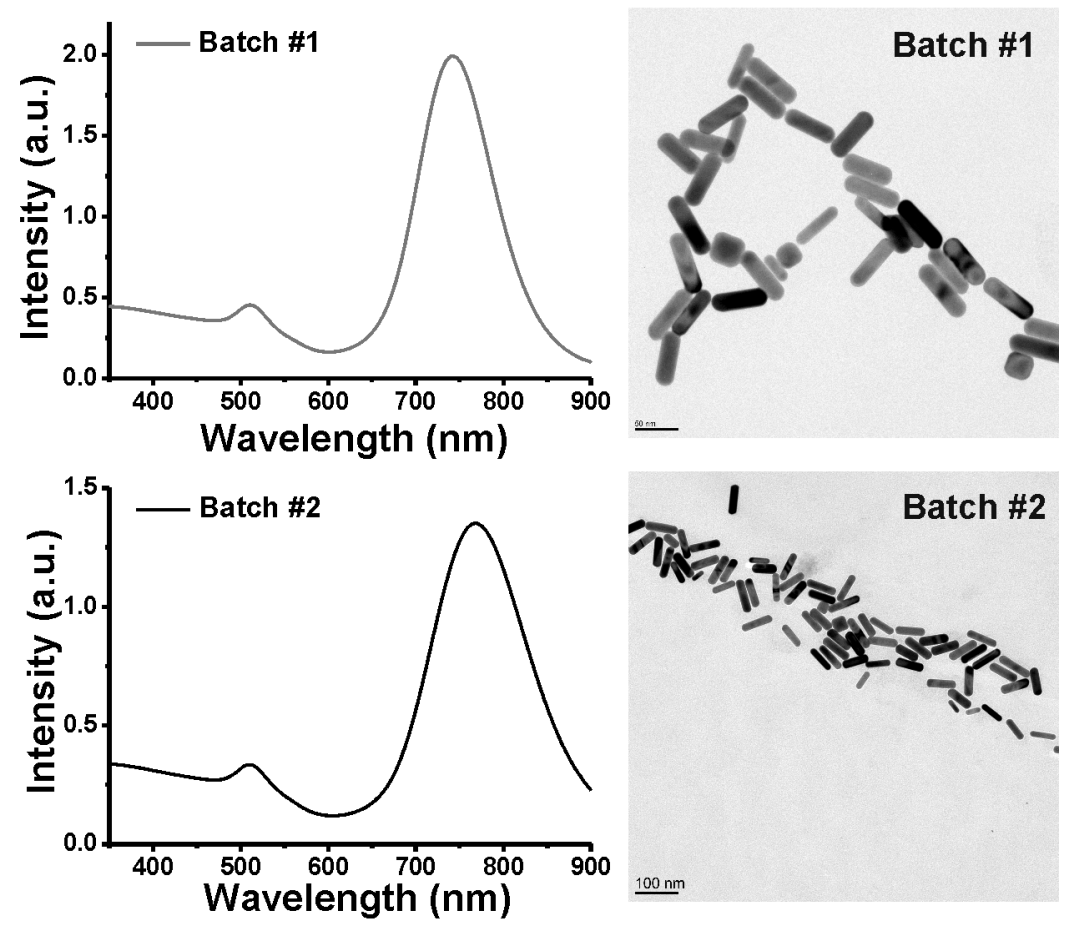

Figure 5.2. UV-Vis spectra (left) and TEM images (right) of Batch \#1 and Batch \#2 AuNRs. Scale bar for Batch \#1 is $50 \mathrm{~nm}$ and for Batch \#2 $100 \mathrm{~nm}$.

The concentration of AuNRs in solution was calculated using the BeerLambert law (Equation 5.1):

$A=\varepsilon \times l \times c$

Where $A$ is the absorbance, I the length of the cuvette $(1 \mathrm{~cm})$ and $\varepsilon$ the molar extinction coefficient $\left(\mathrm{M}^{-1} \mathrm{~cm}^{-1}\right)$. The latter parameter was obtained 
using a calibration plot $^{35}$ where the longitudinal plasmon band peak maximum was used to estimate $\varepsilon$. The calculated concentrations of Batch\#1 and Batch \#2 AuNRs are shown in Table 5.1.

Table 5.1. AuNRs concentrations of Batch \#1 and Batch \#2

\begin{tabular}{|c|c|c|c|c|c|}
\hline Batch \# & $\begin{array}{c}\text { Corrected } \\
\text { Absorbance }\end{array}$ & $\begin{array}{c}\text { Extinction } \\
\text { coefficient } \\
\left(\mathbf{M}^{-1} \mathbf{c m}^{-1}\right)\end{array}$ & $\begin{array}{c}\text { Aspect ratio } \\
(\mathbf{I} / \mathbf{w}) \text { in } \mathbf{~ n m}\end{array}$ & $\begin{array}{c}\lambda_{\max } \\
\text { (nm) }\end{array}$ & $\begin{array}{c}\text { [AuNRs] pM } \\
\text { (in a 10mL } \\
\text { solution) }\end{array}$ \\
\hline $\mathbf{1}$ & 1.86 & $4.120 \times 10^{9}$ & $3.5(63 / 18)$ & 743 & 451 \\
\hline $\mathbf{2}$ & 1.17 & $4.464 \times 10^{9}$ & $3.8(68 / 18)$ & 771 & 262 \\
\hline
\end{tabular}

\subsection{Results and Discussion}

\subsubsection{Functionalization of AuNRs using $G d^{3+}{ }_{-S U D D O 3 A}$ and PEG-SH}

The biocompatibility of AuNRs synthesized using seed-mediated methods is often compromised by the large quantities of CTAB used. However, improved biocompatibility and colloidal stability can be achieved by functionalizing these nanostructures with polyethylene glycol (PEG)-based ligands. ${ }^{23,27,28}$ Here, the biocompatibility and stability of AuNRs coated with various $\mathrm{Gd}^{3+}$-SUDDO3A:PEG-SH percentage ratios were studied (Figure 5.3); thereby allowing us to understand the influence of the $\mathrm{Gd}^{3+}$ SUDDO3A coating on the stability, biocompatibility and attainable $\mathrm{Gd}^{3+}$ loading of the AuNRs. This was done by incubating $1 \mathrm{~mL}$ of Batch \#1 AuNRs $\left([A u N R]_{\text {stock solution }}=900 \mathrm{pM}\right)$ with $5 \mathrm{mM} \mathrm{Gd}^{3+}$-SUDDO3A (filtered with a $0.2 \mu \mathrm{m}$ filter before using) and $5 \mathrm{mM}$ PEG-SH at 5 different volume percentages (Table 5.2). For all solutions, tris(2-carboxyethyl)phosphine (TCEP, $5 \mathrm{mg}$ ) was added in order to both break any disulfide bonds and to prevent their formation. After incubation for 2 days, all 5 solutions were centrifuged 3 times at 11.000 rpm for 15 minutes (in PBS buffer, $\mathrm{pH}$ 7.5), in 
order to remove excess $\mathrm{Gd}^{3+}$-SUDDO3A. The final pellets were resuspended in PBS $+5 \%$ BSA.
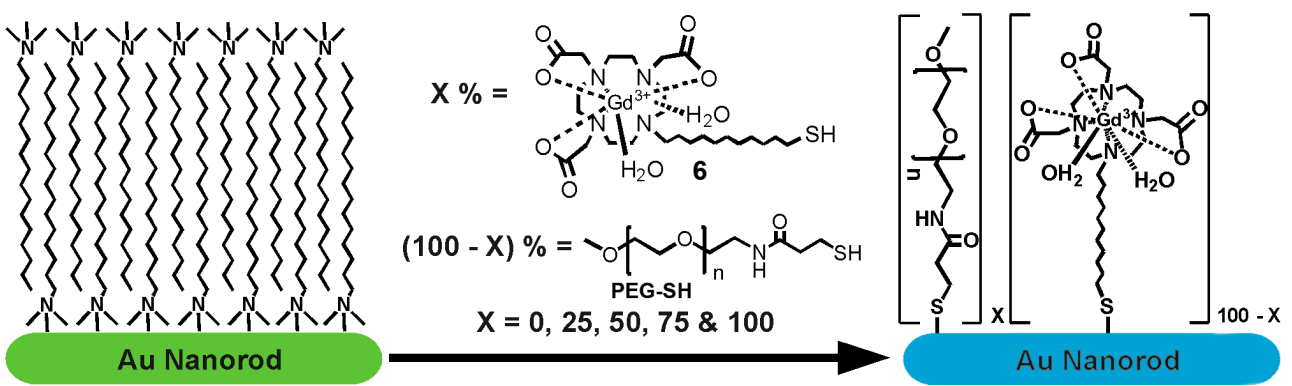

Figure 5.3. AuNRs ligand exchange using different $\mathrm{Gd}^{3+}-$ SUDDO3A:PEG-SH (volume) percentages, expressed as $\% X$ and \% (100 - X), respectively. The localization, size and distribution of the ligands along the AuNRs are only indicative.

Table 5.2. Quantities (volumes) of $5 \mathrm{mM} \mathrm{Gd}^{3+}-$ SUDDO3A and $5 \mathrm{mM}$ PEG-SH used to incubate $1 \mathrm{~mL}$ of Batch \#1 AuNRs

\begin{tabular}{|c|c|c|}
\hline $\mathrm{Gd}^{3+}$-SUDDO3A:PEG-SH (\%) & $\mathrm{mL} \mathrm{Gd}^{3+}$-SUDDO3A & $\mathrm{mL}$ PEG-SH \\
\hline $\mathbf{0 : 1 0 0}$ & 0 & 5 \\
\hline $\mathbf{2 5 : 7 5}$ & 1.25 & 3.75 \\
\hline $\mathbf{5 0 : 5 0}$ & 2.5 & 2.5 \\
\hline $\mathbf{7 5 : 2 5}$ & 3.75 & 1.25 \\
\hline $\mathbf{1 0 0 : 0}$ & 5 & 0 \\
\hline
\end{tabular}

The newly coated AuNRs were characterized using UV-Vis absorbance spectroscopy and TEM analysis. In most of the cases, the AuNRs exhibited a red-shifted longitudinal plasmon band peak maximum upon ligand exchange by incubation using various $\mathrm{Gd}^{3+}$-SUDDO3A:PEG-SH percentages (Figure 5.4, left). While the largest red shifts are observed in the AuNRs incubated with the highest $\mathrm{Gd}^{3+}$-SUDDO3A percentage (pink and grey lines), those incubated with 50:50\% showed almost no shift in the absorption spectra (green line). In our hands, we have constantly observed this dissimilarity when using this ligand exchange protocol. The 
broadened longitudinal plasmon bands observed in all cases indicate modified colloidal stabilities as the coating composition of the AuNRs changes. As expected, the lowest colloidal stability is observed in the AuNRs incubated with only $\mathrm{Gd}^{3+}$-SUDDO3A (grey line), as these nanostructures tend to flocculate/aggregate rapidly. Furthermore, no changes in the morphology of the functionalized AuNRs are observed, as demonstrated by TEM (Figure 5.4, right). Overall, higher colloidal stability was observed at decreasing $\mathrm{Gd}^{3+}$-SUDDO3A incubation percentages. Therefore, under the studied conditions, a 25:75\% $\mathrm{Gd}^{3+}$-SUDDO3A:PEG$\mathrm{SH}$ incubation ratio leads to the most stable assemblies.
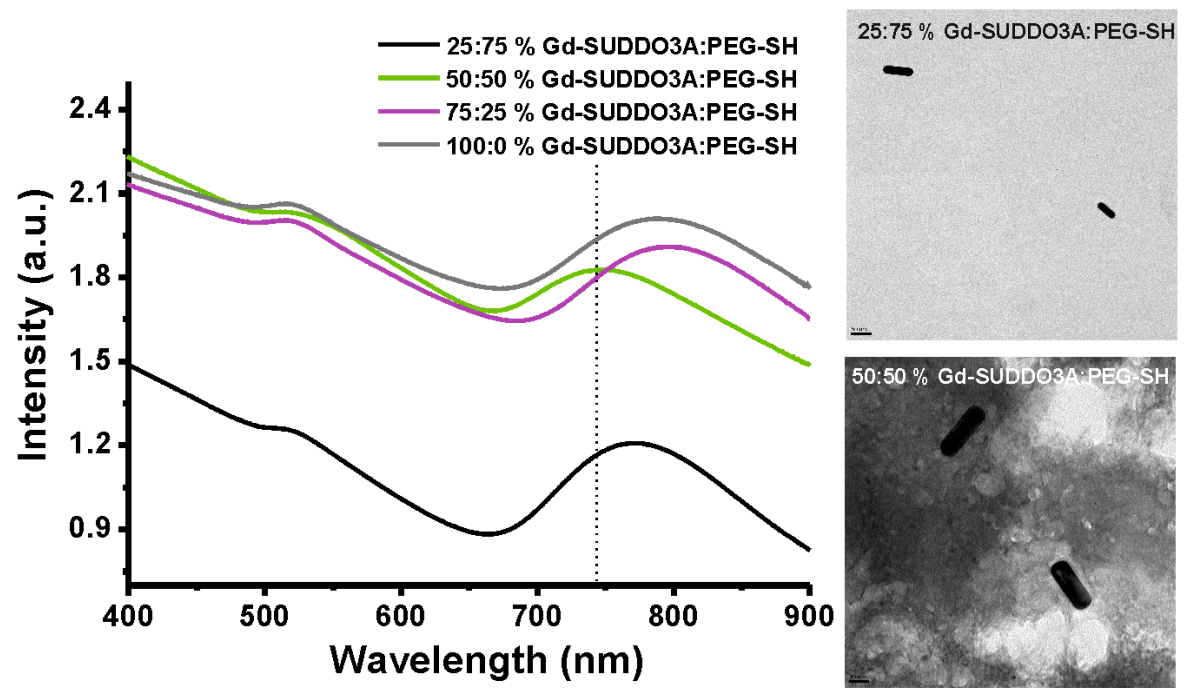

Figure 5.4. Left: UV-Vis spectrum (in PBS + 5\% BSA) of AuNRs incubated with different $\mathrm{Gd}^{3+}$-SUDDO3A:PEG-SH percentage ratios. Dashed line represents the longitudinal plasmon band peak maximum of Batch \#1 at $744 \mathrm{~nm}$. Right: TEM images of 25:75 (top) and 50:50 (bottom) \% Gd ${ }^{3+}$-SUDDO3A:PEG-SH incubated AuNRs showing that the morphology of the AuNRs does not change after ligand exchange. Note the background contrast in the $50: 50 \%$ picture given by the combination of albumin, salts from buffer and $\mathrm{Gd}^{3+}-$ SUDDO3A surrounding the AuNRs as the nanostructures collapse upon appliance on the TEM grid. Scale bar is $50 \mathrm{~nm}$ and $20 \mathrm{~nm}$ respectively. 
In previous studies, the $\mathrm{Gd}^{3+}$-to-Au ratio (in weight) of functionalized $\mathrm{AuNRs}^{24}$ and $\mathrm{Au}$ nanoparticles ${ }^{12}$ has been successfully studied using inductively coupled plasma (ICP)-based techniques, namely ICP-AES and ICP-MS, respectively. In this way, the degree of functionalization of the final nanostructures was quantified. Here, in order to quantify both the $\mathrm{Gd}^{3+}$ and $\mathrm{Au}$ concentrations, all samples were analyzed using total reflection X-ray fluorescence (TXRF). The average volume of a single AuNR was calculated assuming ideal cylinder nanorods (Equation 5.2). ${ }^{24}$ The number of Au atoms per AuNR and the number of AuNRs were calculated using Equation 5.3 and Equation 5.4, respectively.

$V_{N R}=\pi r^{2} l$

\# Au atoms per AuNR $=\rho_{A u} V_{N R}$

$\# A u N R s=\frac{\# \text { Au atoms }}{\# \text { atoms per AuNR }}$

Where $\rho_{A u}$ is 59 atoms $/ \mathrm{nm}^{3}$ (FCC structure) ${ }^{36}$ and $\mathrm{r}$ is half the width and $I$ the length of the NRs. Based on TXRF, the incubation of AuNRs with various $\mathrm{Gd}^{3+}$-SUDDO3A:PEG-SH ratios led to successful thiol-based ligand exchange as increasing $\mathrm{Gd}^{3+}$ ions were detected (Table 5.3). Furthermore, as the $\mathrm{Gd}^{3+}$-SUDDO3A incubation percentages increased, higher $\mathrm{Gd}^{3+}: \mathrm{Au}$ ratios (weight:weight) were obtained. Surprisingly, the calculated number of $\mathrm{Gd}^{3+}$ ions per AuNR is 40 to 90 times higher than previously reported AuNRs functionalized with $\mathrm{Gd}^{3+}$-DTPA-based ligands. ${ }^{24}$ However, this number decreases when calculating the AuNR concentration using the Beer-Lambert law (section 5.2.3, Table 5.3). More specifically, the maximal $\mathrm{Gd}^{3+}$ per AuNR loading is reached at an incubation 
percentage of 75:25 $\mathrm{Gd}^{3+}$-SUDDO3A:PEG-SH. This discrepancy in the estimation of the AuNR concentration, leading to differences in the calculated $\mathrm{Gd}^{3+}$ per AuNR loadings, suggests a sensitivity/detection issue when using TXRF for the determination of the Au concentration which might arise from the presence of a large organic matrix (BSA). ${ }^{37}$

Table 5.3. Elemental analysis data for all incubated AuNRs

\begin{tabular}{|c|c|c|c|c|}
\hline Gd $^{3+}$-SUDDO3A:PEG-SH (\%) & $25: 75$ & $50: 50$ & $75: 25$ & $100: 0$ \\
\hline \multicolumn{5}{|c|}{ From TXRF Data } \\
\hline Ratio $\mathrm{Gd}^{3+}: \mathrm{Au}(\mathrm{w} / \mathrm{w})$ & $100: 6.5$ & $100: 4.1$ & $100: 3.2$ & $100: 2.7$ \\
\hline$\left[\mathrm{Gd}^{3+}\right](\mathrm{mM})$ & 1.9 & 3.6 & 4.8 & 4.2 \\
\hline [Au] (M) & $9.6 \times 10^{-5}$ & $11.8 \times 10^{-5}$ & $12.5 \times 10^{-5}$ & $8.9 \times 10^{-5}$ \\
\hline \# atoms $\mathrm{Au}$ & $5.8 \times 10^{19}$ & $7.1 \times 10^{19}$ & $7.5 \times 10^{19}$ & $5.4 \times 10^{19}$ \\
\hline Volume one AuNR (nm) & 16023 & 16023 & 16023 & 16023 \\
\hline \# atoms per AuNR & 945382 & 945382 & 945382 & 945382 \\
\hline \# AuNRs & $6.1 \times 10^{13}$ & $7.5 \times 10^{13}$ & $8 \times 10^{13}$ & $5.7 \times 10^{13}$ \\
\hline \# $\mathrm{Gd}^{3+}$ atoms & $1.1 \times 10^{21}$ & $2.2 \times 10^{21}$ & $2.9 \times 10^{21}$ & $2.5 \times 10^{21}$ \\
\hline \# Gd ${ }^{3+}$ ions per AuNR & $1.8 \times 10^{7}$ & $2.9 \times 10^{7}$ & $3.6 \times 10^{7}$ & $4.4 \times 10^{7}$ \\
\hline \multicolumn{5}{|c|}{ From UV Data (AuNR concentration) and TXRF (Gd ${ }^{3+}$ concentration) } \\
\hline \# AuNRs & $1.2 \times 10^{14}$ & $1.2 \times 10^{14}$ & $1 \times 10^{14}$ & $1 \times 10^{14}$ \\
\hline$\# \mathrm{Gd}^{3+}$ atoms & $1.1 \times 10^{21}$ & $2.2 \times 10^{21}$ & $2.9 \times 10^{21}$ & $2.5 \times 10^{21}$ \\
\hline \# Gd ${ }^{3+}$ ions per AuNR & $9.3 \times 10^{6}$ & $1.9 \times 10^{7}$ & $2.9 \times 10^{7}$ & $2.5 \times 10^{7}$ \\
\hline
\end{tabular}

While high $\mathrm{Gd}^{3+}$ loadings are expected when using such nanostructures due to their large surface to volume ratios, the higher values obtained strongly suggests the formation of highly dense "paramagnetic networks" of $\mathrm{Gd}^{3+}-\mathrm{SUDDO} 3 \mathrm{~A}$ molecules surrounding the AuNRs and possibly entrapped in the long PEG chains. The formation of such highly dense coatings around the AuNRs is likely to occur, as the amphiphilic $\mathrm{Gd}^{3+}$ SUDDO3A ligand used is known to self-assemble in water, with a critical aggregation concentration (cac) of $0.63 \mathrm{mM}$ (Appendix 5.1). Therefore, it is thinkable that multiple layers can form around the AuNRs as the $\mathrm{Gd}^{3+}$ - 
SUDDO3A concentration increases. This observation is in line with previously studied AuNRs, where the formation of thick, non-uniform polymer shells surrounding AuNRs was observed. ${ }^{31}$ In the absence of PEG-SH, these highly dense "paramagnetic packing" around the AuNRs is evident from the low colloidal stability AuNRs show as the water solubility of $\mathrm{Gd}^{3+}-$ SUDO3A is lower than that of PEG-SH. Moreover, the lower $\mathrm{Gd}^{3+}$ per AuNR loading obtained for the 100:0\% Gd ${ }^{3+}$-SUDDO3A:PEG-SH incubated sample (when using UV data for the calculation of the AuNR concentration) strongly suggest the need for a PEG-SH in order to entrap more $\mathrm{Gd}^{3+}-$ SUDDO3A molecules. However, direct observation of any network of molecules using, e.g., TEM, is challenging since the sample preparation is a critical factor. Most likely, these densely packed paramagnetic networks will tend to collapse during sample preparation thereby leading to dark backgrounds consisting of organic (albumin, $\mathrm{Gd}^{3+}$ SUDDO3A) and inorganic (salts from buffer) matter (as observed in Figure 5.4 , right bottom).

The biocompatibility of $\mathrm{Gd}^{3+}$-SUDDO3A:PEG-SH coated AuNRs was evaluated by studying the viability of $\mathrm{C} 2 \mathrm{C} 12$ mouse myoblast cells upon incubation for $24 \mathrm{~h}$ with 4 serial dilutions of the 5 different $\mathrm{Gd}^{3+}$ SUDDO3A:PEG-SH coated AuNRs ([AuNRs $\left.]_{\text {stock solution }}=200 \mathrm{pM}\right)$ in triplicate. As shown in Figure 5.5, cells incubated with only PEG-SH coated AuNRs (pink line) show the highest survival percentage at the concentration rage studied, followed by those incubated with $25 \% \mathrm{Gd}^{3+}$ SUDDO3A (blue line). On the other hand, cells incubated with $100 \% \mathrm{Gd}^{3+}$ SUDDO3A coated AuNRs showed the lowest median lethal concentration $\left(\mathrm{LC}_{50}, 50 \mathrm{pM}\right)$ upon $24 \mathrm{~h}$ exposure (black line) while, for all of the other cases, this value was higher than $100 \mathrm{pM}$. These results confirm that the biocompatibility of the AuNRs studied is lowered as the $\mathrm{Gd}^{3+}$-SUDDO3A incubation percentage increases. 


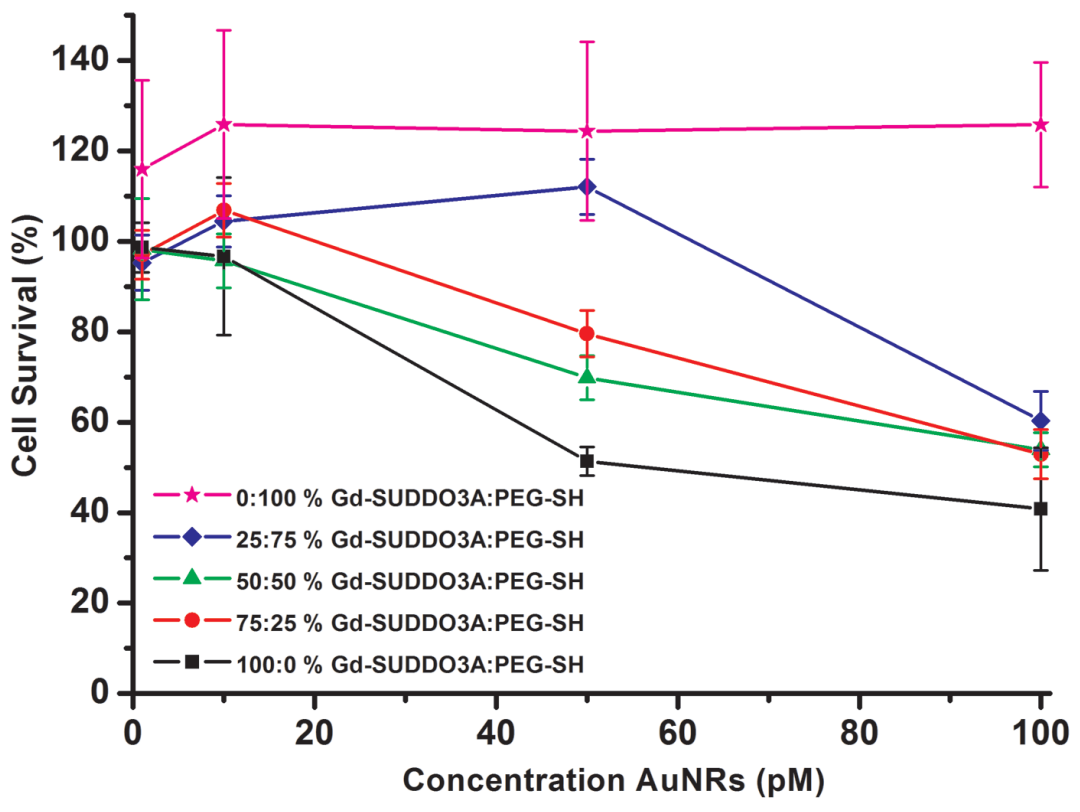

Figure 5.5. Mouse myoblast $\mathrm{C} 2 \mathrm{C} 12$ cell response upon exposure to $\mathrm{Gd}^{3+}$-SUDDO3A:PEG$\mathrm{SH}$ coated AuNRs. Cells were incubated for $24 \mathrm{~h}$ with different $\mathrm{Gd}^{3+}-$ SUDDO3A:PEG-SH percentages at increasing AuNR concentrations. For comparison purposes, response upon exposure to PEG-SH coated AuNRs is also shown (pink line).

5.3.2 MMAuNRs: Functionalization of AuNRs using Gd ${ }^{3+}{ }_{-} S U D D O 3 A$, $P E G-S H$ and Biotin-SH

The incorporation of the second imaging modality to AuNRs was achieved using the biotin-streptavidin system. Here, AuNRs were biotinylated by incubation with $\mathrm{Gd}^{3+}$-SUDDO3A (6), PEG-SH and Biotin-SH (8, Figure 5.6) at different ratios. As described in previous sections, the (optimum) $\mathrm{Gd}^{3+}-$ SUDOD3A percentage was $25 \%$. The AuNRs were prepared by incubating $1 \mathrm{~mL}$ of Batch \#2 AuNRs ([AuNR $]_{\text {stock solution }} 873 \mathrm{pM}$ ) with $5 \mathrm{mM} \mathrm{Gd}^{3+}$ SUDDO3A (filtered before using) and $5 \mathrm{mM}$ PEG-SH and $5 \mathrm{mM}$ Biotin-SH (filtered before using) at 3 different volume percentages (quantities shown in Table 5.4). For all solutions, tris(2-carboxyethyl)phosphine (TCEP, $5 \mathrm{mg}$ ) 
was added in order to both brake any disulfide bonds and to prevent their formation. After incubation for 2 days, the 3 solutions were centrifuged 3 times at $11.000 \mathrm{rpm}$ for 15 minutes (in PBS buffer, $\mathrm{pH} 7.5$ ) and resuspended in PBS buffer $+5 \%$ BSA. The fluorescent dye was incorporated into the system by incubation with $0.6 \mu \mathrm{M}$ Streptavidin Cy5 for one hour. Once again, the samples were centrifuged 3 times at 11.000 rpm for 15 minutes, in order to remove excess $\mathrm{Gd}^{3+}$-SUDDO3A. The final pellets were resuspended in PBS $+5 \%$ BSA.
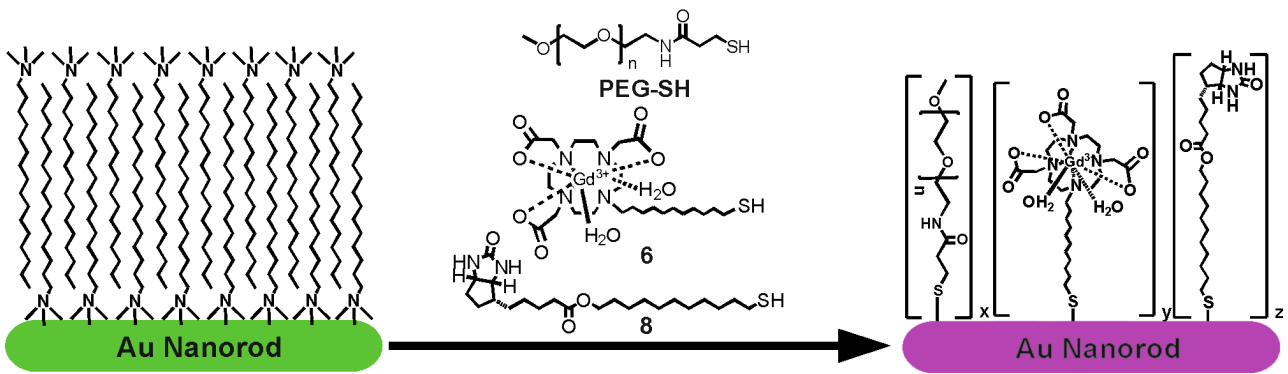

Figure 5.6. Schematic representation of multimodal gold nanorods (MMAuNRs), prepared by simultaneous thiol-based ligand exchange, using optimal percentages of PEG-SH (x), $\mathrm{Gd}^{3+}-\mathrm{SUDDO} 3 \mathrm{~A}(\mathrm{y})$ and Biotin-SH (z). The localization, size and distribution of the ligands along the AuNRs are only indicative.

Table 5.4. Quantities (volumes) of $5 \mathrm{mM} \mathrm{Gd}^{3+}$-SUDDO3A, $5 \mathrm{mM}$ PEG-SH and 5mM Biotin$\mathrm{SH}$ used to incubate $1 \mathrm{~mL}$ of Batch \#2 AuNRs

\begin{tabular}{|c|c|c|c|}
\hline $\begin{array}{c}\mathrm{Gd}^{3+} \text {-SUDDO3A:PEG-SH:Biotin-SH } \\
(\%)\end{array}$ & $\begin{array}{c}\mathrm{mL} \mathrm{Gd}^{3+}- \\
\text { SUDDO3A }\end{array}$ & $\begin{array}{c}\mathrm{mL} \text { PEG- } \\
\text { SH }\end{array}$ & $\begin{array}{c}\mathrm{mL} \text { Biotin- } \\
\text { SH }\end{array}$ \\
\hline $\mathbf{0 : 1 0 0 : 0}$ & 0 & 7 & 0 \\
\hline $\mathbf{0 : 5 0 : 5 0}$ & 0 & 3.5 & 3.5 \\
\hline $\mathbf{2 5 : 5 0 : 2 5}$ & 2.33 & 2.33 & 2.33 \\
\hline
\end{tabular}


After ligand exchange of the AuNRs using various $\mathrm{Gd}^{3+}$-SUDDO3A:PEG$\mathrm{SH}$ :Biotin-SH percentage ratios, a red shift of the peak corresponding to the longitudinal plasmon band occurred (Figure 5.7, left). Furthermore, no changes in this peak are observed after incubation with Streptavidin Cy5 (black line). The higher colloidal stability of these nanostructures, when compared to those incubated with only $\mathrm{Gd}^{3+}-$ SUDDO3A and PEG-SH (Figure 5.4, left), is evident from the smaller broadening of the longitudinal plasmon bands observed. No changes in the morphology of these ligand exchanged-AuNRs are observed by TEM either before (top) or after (bottom) incubation with Streptavidin Cy5 (Figure 5.7, right).
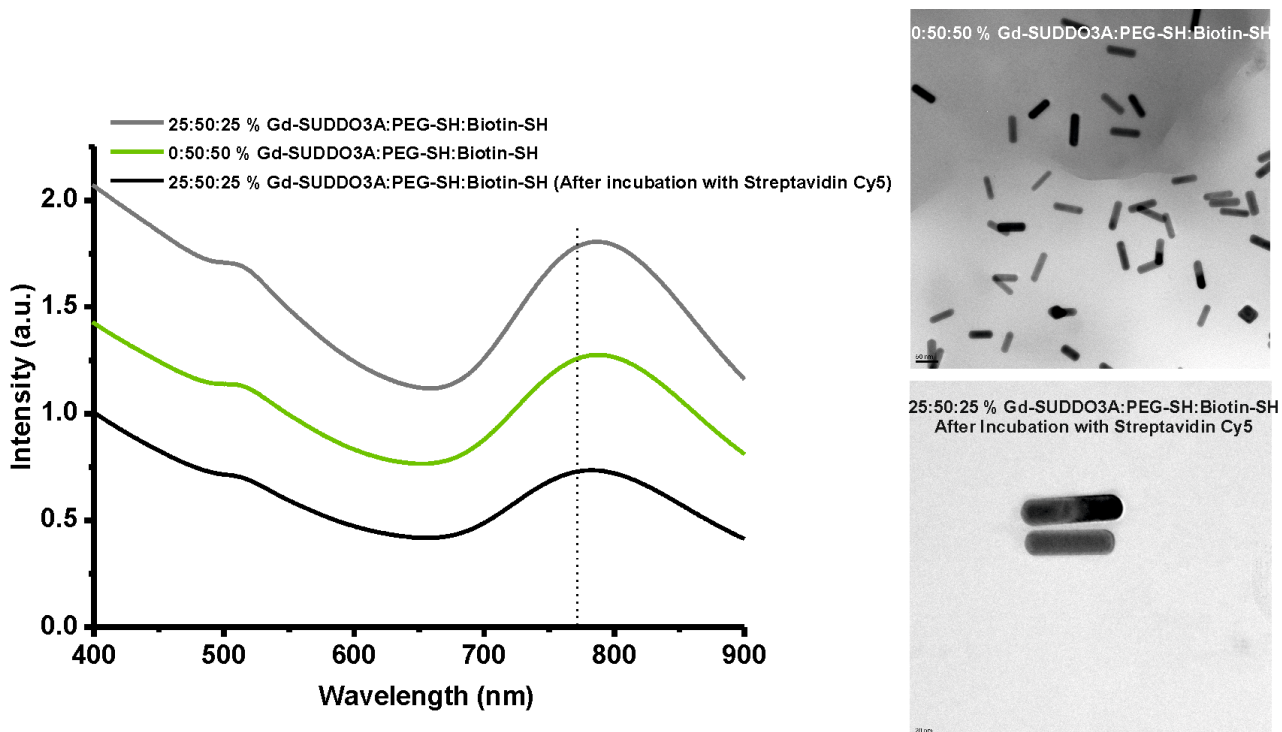

Figure 5.7. Left: UV-Vis spectrum (in PBS + 5\% BSA) of AuNRs incubated with different percentages of $\mathrm{Gd}^{3+}$-SUDDO3A:PEG-SH:Biotin-SH. Dashed line represents the longitudinal plasmon band peak maximum of Batch \#2 at $771 \mathrm{~nm}$. Right: TEM images of 0:50:50 (top) and 25:50:25 (bottom) \% Gd ${ }^{3+}$-SUDDO3A:PEG-SH:Biotin-SH incubated AuNRs showing that the morphology of the AuNRs does not change either after ligand exchange or subsequent incubation with Streptavidin-Cy5. Scale bar is $50 \mathrm{~nm}$ and $20 \mathrm{~nm}$, respectively. 
Analogous to the AuNRs previously studied, the $\mathrm{Gd}^{3+}$ concentration was assessed using TXRF analysis, while the AuNR concentration was obtained from UV data (section 5.2.3). After incubation of AuNRs using 25:50:25 Gd $^{3+}$-SUDDO3A:PEG-SH:Biotin-SH percentage ratios (and subsequent incubation with Streptavidin Cy5), successful ligand exchange was confirmed by the detection of $\mathrm{Gd}^{3+}$ ions. Curiously, the number of $\mathrm{Gd}^{3+}$ ions per AuNR observed is only 4 times higher than that reported for non-covalently functionalized paramagnetic $A_{u N R s^{24}}\left(2.1 \times 10^{6}\right.$ vs. $5.1 \times 10^{5}$ ) and lower than that measured for the $75: 25 \mathrm{Gd}^{3+}$ SUDDO3A:PEG-SH sample $\left(2.1 \times 10^{6}\right.$ vs. $9.3 \times 10^{6}$, respectively). Analogous to previously synthesized AuNRs, the formation of a highly dense paramagnetic $\mathrm{Gd}^{3+}$-SUDDO3A layer around the AuNRs is thought to be responsible for the higher $\mathrm{Gd}^{3+}$ loadings observed.

The biocompatibility of the biotinylated AuNRs was studied in vitro by incubating $\mathrm{C} 2 \mathrm{C} 12$ cells with 5 serial dilutions of the 3 different AuNR samples (Table 5.5) in triplicate. As shown in Figure 5.8, the maximum percentage of cell survival after $24 \mathrm{~h}$ of exposure corresponded to cells incubated with 0:50:50\% $\mathrm{Gd}^{3+}-$ SUDDO3A:PEG-SH-Biotin-SH (red line). Moreover, AuNRs incubated with 25 and 50\% Biotin-SH showed median lethal concentrations $\left(\mathrm{LC}_{50}\right)$ higher than $150 \mathrm{pM}$, both higher than that obtained for AuNRs incubated with only $\mathrm{Gd}^{3+}$-SUDDO3A and PEG-SH (green/red and blue lines, respectively). Whereas previous cell viability studies showed that AuNRs incubated with 25\% Gd $\mathrm{Gd}^{3+}$-SUDDO3A showed an $\mathrm{LC}_{50}$ value higher than $100 \mathrm{pM}$ (blue line), the incorporation of the biotin moiety results in an even higher $\mathrm{LC}_{50}$ value (> $\left.150 \mathrm{pM}\right)$. This increase in cell viability percentage is not surprising, since biotin is natively found in many biological systems with negligible toxicity. Moreover, the $\mathrm{LC}_{50}$ values obtained are comparable to previously reported PEGylated AuNRs (> 165 $\mathrm{pM}){ }^{28}$ 


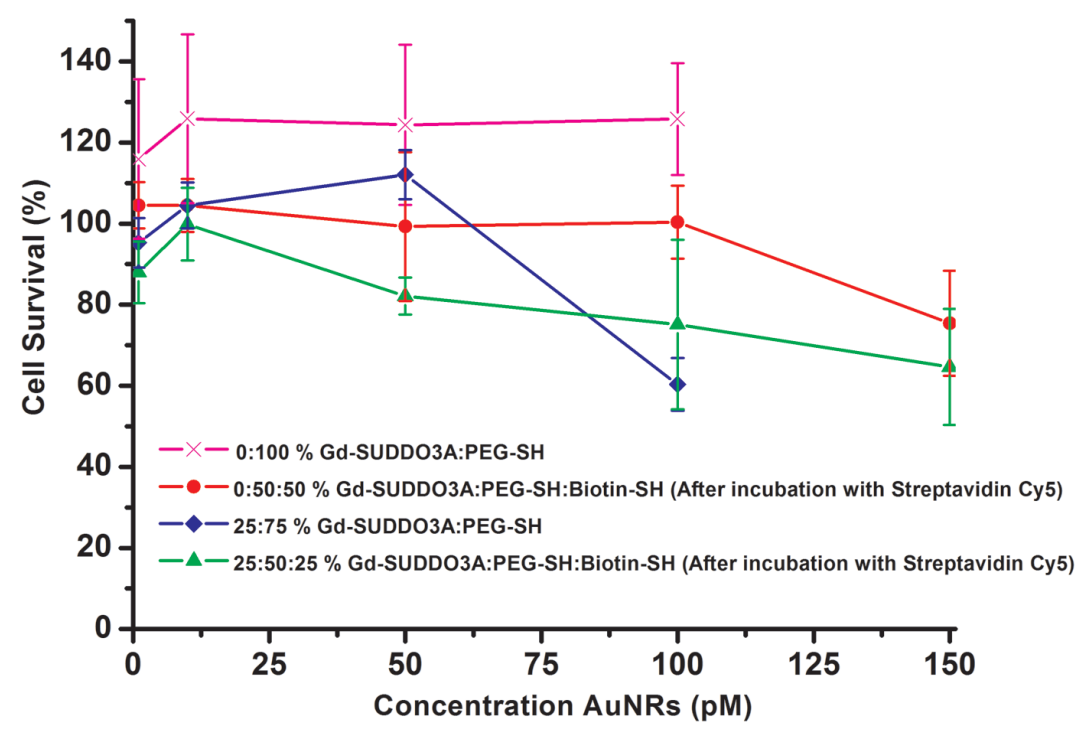

Figure 5.8. Mouse myoblast $\mathrm{C} 2 \mathrm{C} 12$ cell response upon exposure to $\mathrm{Gd}^{3+}$-SUDDO3A:PEG$\mathrm{SH}$ :Biotin-SH coated AuNRs. Cells were incubated for $24 \mathrm{~h}$ with different $\mathrm{Gd}^{3+}-$ SUDDO3A:PEG-SH:Biotin-SH percentages at increasing AuNR concentrations. For comparison purposes, cell response upon exposure to $\mathrm{Gd}^{3+}$-SUDDO3A:PEG-SH and PEG$\mathrm{SH}$ coated AuNRs is also shown (pink line).

\subsubsection{Properties of MMAuNRS}

Cy 5 fluorescence quenching after functionalization of the AuNRs is expected, as previous reports using fluorescein have shown a dramatic decrease of its quantum yield after AuNR functionalization. ${ }^{31}$ Nevertheless, the residual fluorescence proved to be sufficient for imaging applications. Therefore, the fluorescent MMAuNRs were visualized in vitro using $\mathrm{C} 2 \mathrm{C} 12$ cells incubated with 150 pM MMAuNRs. After exposure for $24 \mathrm{~h}$ to these nanostructures, the cells were stained with Alexa Fluor 488 (green) and DAPI (blue) in order to elucidate their location in the wells. As shown in Figure 5.9 (top), fluorescence linked to both the cells (left image) and the MMAuNRs (center image) was successfully observed. While cell 
internalization of the MMAuNRs remains inconclusive, the overlay of these two images (right image) shows that the MMAuNRs are colocalized in the surroundings of the cells. For control purposes, wells without incubated cells were also treated with the same staining procedure and the same MMAuNR solution. As Figure 5.9 (bottom) shows, fainted red fluorescence (corresponding to the MMAuNRs) was observed. This lower fluorescence intensity observed (even at higher exposure times) strongly suggests that the MMAuNRs are not colocalized and clustered due to the absence of cells.
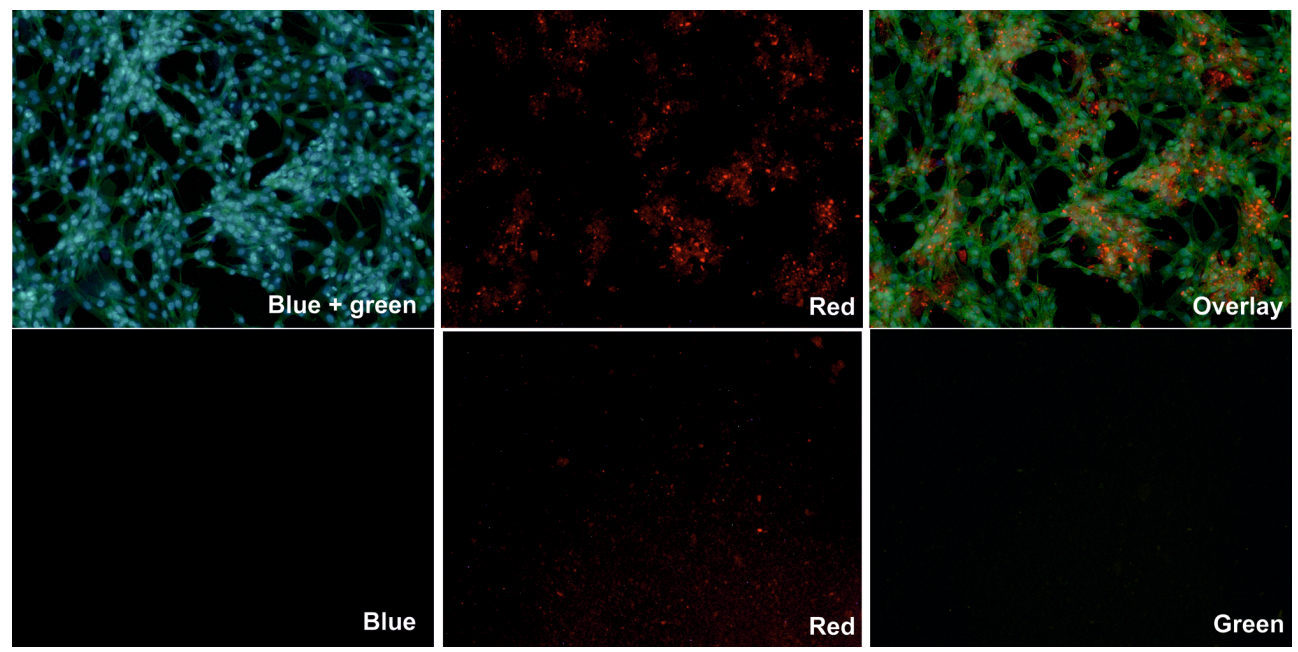

Figure 5.9. Top: fluorescence microscopy images of $\mathrm{C} 2 \mathrm{C} 12$ cells incubated with MMAuNRs. Cells were stained using Alexa Fluor ${ }^{\circledR} 488$ (cytoskeleton, green, exposure time $50 \mathrm{~ms}$ ) and DAPI (cell nuclei, blue, exposure time $1 \mathrm{~ms})$. Left: Overlay of blue + green filters, showing the location of the cells in the well. Center: red emission observed corresponding to Cy5-labeled MMAuNRs (exposure time $2000 \mathrm{~ms}$ ). Right: overlay of the previously described images. Bottom: control experiments in wells where no cells were incubated (blue, red and green filter). The blue and green filter images confirm the absence of cells, while the red fluorescence confirms the presence of MMAuNRs all over the well (exposure time $15000 \mathrm{~ms}$ ). 
Finally, the MR contrast provided by the MMAuNRs is evident from the $T_{1}$ weighted MR image shown in Figure 5.10, highlighting the differences in contrast observed at different $\mathrm{Gd}^{3+}$ concentrations (and with respect to PBS buffer). In addition, the relaxation efficiency of MMAuNRs was assessed by measuring the longitudinal relaxation times $\left(T_{1}\right)$ of a series of dilutions (of known $\mathrm{Gd}^{3+}$ concentration determined by TXRF) using a standard saturation recovery experiment at $600 \mathrm{MHz}$ (14.1 T). By plotting the measured $1 / T_{1}$ values as a function of $\mathrm{Gd}^{3+}$ concentration, the relaxivity $r_{1}\left(\mathrm{mM}^{-1} \mathrm{~s}^{-1}\right)$ was obtained as the slope of the curve. For control purposes, the ionic relaxivity of the $\mathrm{Gd}^{3+}$-SUDDO3A complex in solution was also calculated $\left(r_{1}=0.81 \mathrm{mM}^{-1} \mathrm{~s}^{-1}\right.$, Appendix 5.2).

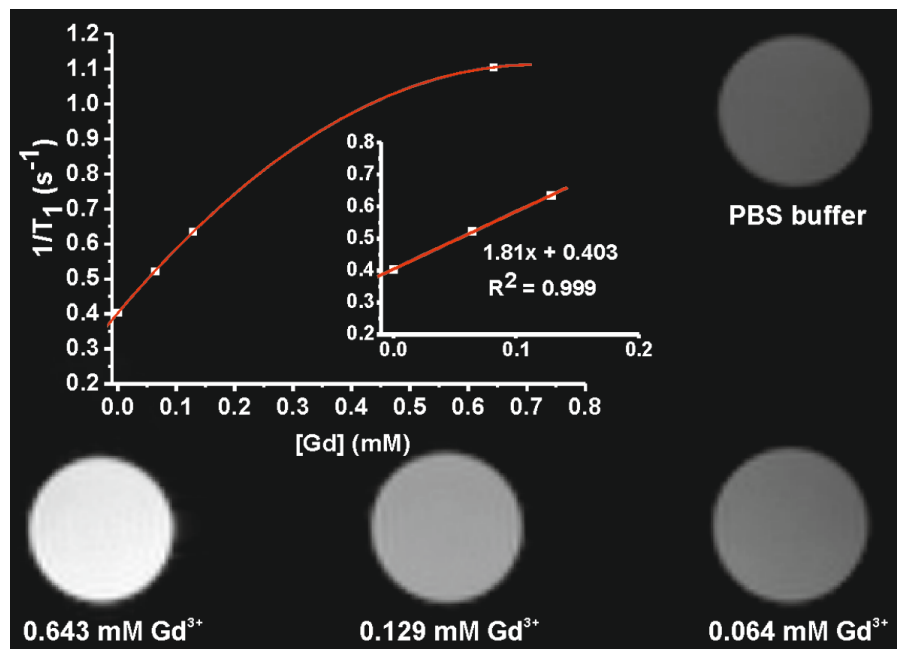

Figure 5.10. $T_{1}$-weighted MR image of MMAuNRs at different $\mathrm{Gd}^{3+}$ concentrations (600 $\mathrm{MHz}, \mathrm{TE}=4 \mathrm{~ms}, \mathrm{TR}=500 \mathrm{~ms}$ ). The plot shows ionic $r_{1}$ relaxivity corresponding to the MMAuNRs. The inset plot corresponds to a linear fitting of the 0-0.13 $\mathrm{mM} \mathrm{Gd}^{3+}$ concentration range $\left(r_{1}=1.8 \mathrm{mM}^{-1} \mathrm{~s}^{-1}\right)$.

The non-linearity observed in the $1 / T_{1}$ vs. $\left[\mathrm{Gd}^{3+}\right]$ concentration plot strongly suggest $T_{1}$ saturation, ${ }^{8,38}$ as the former value approaches a plateau at $\mathrm{Gd}^{3+}$ 
concentrations between 0.13 and $0.6 \mathrm{mM}$ (Figure 5.10). This behavior has previously been observed in AuNRs functionalized with $\mathrm{Gd}^{3+}$-DTPA complexes at $\mathrm{Gd}^{3+}$ concentrations higher than $1.17 \mathrm{mM}^{24}$ Furthermore, a relaxivity plot in the $\mathrm{Gd}^{3+}$ concentration range $0-0.13 \mathrm{mM}$ (inset Figure 5.10) shows enhanced ionic $r_{1}$ relaxivity for the MMAuNRs when compared to the $\mathrm{Gd}^{3+}$-SUDDO3A complex in solution $\left(1.8 \mathrm{mM}^{-1} \mathrm{~s}^{-1}\right.$ vs. $0.81 \mathrm{mM}^{-1} \mathrm{~s}^{-1}$, respectively). While an increase in ionic $r_{1}$ relaxivity is expected due to slower global rotational motion $\left(\tau_{R}\right)$ upon attachment to the AuNRs, the rather modest enhancement observed may be due to the molecular crowding resulting from the formation highly dense "paramagnetic networks" of attached and non-attached $\mathrm{Gd}^{3+}$-SUDDO3A molecules (in the form of free, non-aggregated molecules, micelles, etc) around the surface of the AuNRs (Figure 5.11). While an increase in relaxivity is expected when $\mathrm{Gd}^{3+}$-SUDDO3A molecules are attached to the AuNR or in an aggregated form, this is not the case for the relaxivity of non-aggregated, entrapped by the long PEG chains, $\mathrm{Gd}^{3+}$-SUDDO3A molecules. Moreover, in these densely packed networks, relaxivity is likely to be affected by the limited water exchange and diffusion due to low accessibility of the paramagnetic moieties, as previous studies in hybrid silica nanoparticles have shown. ${ }^{39}$ 


$$
\zeta=G d^{3+}-S U D D O 3 A
$$
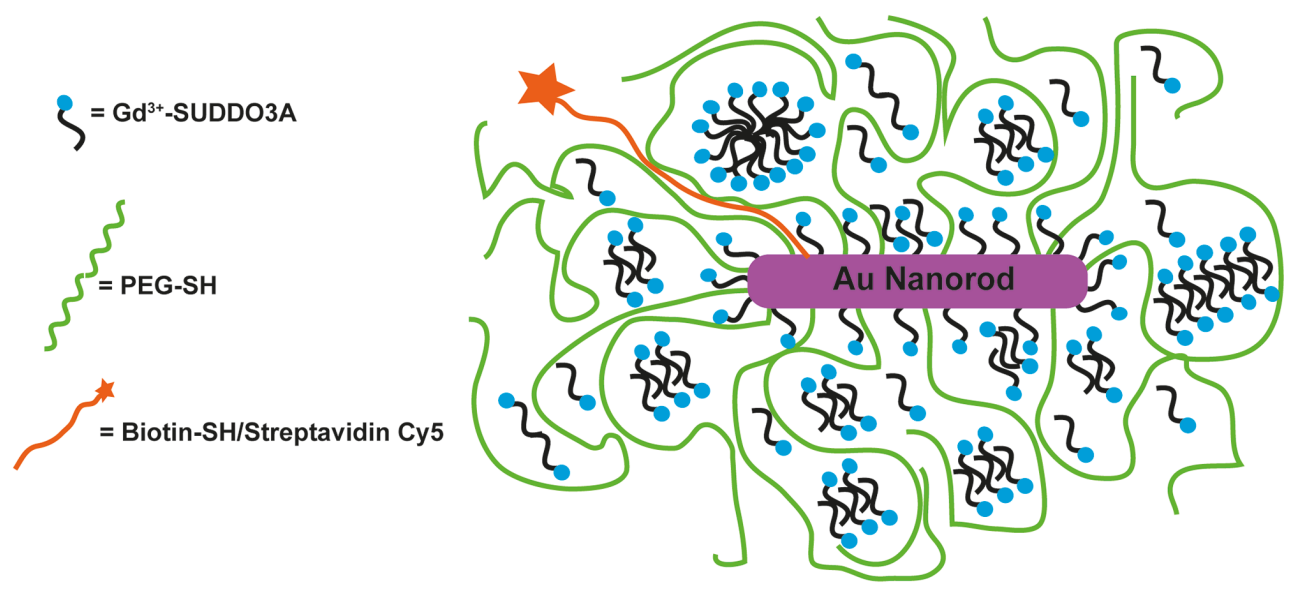

Figure 5.11. Schematic representation of the suspected highly dense paramagnetic network formed around the AuNRs, showing both attached and unattached $\mathrm{Gd}^{3+}$ SUDDO3A molecules.

With respect to the relaxivity per AuNR, defined as the ionic relaxivity $r_{1}$ times number of calculated $\mathrm{Gd}^{3+}$ ions per AuNR, this value is estimated to be $4 \times 10^{6} \mathrm{mM}^{-1} \mathrm{~s}^{-1}$, which is lower than previously reported values for paramagnetic labeled AuNRs. ${ }^{24}$ However, this value is much lower when calculated as the slope of the $1 / T_{1}$ vs. [AuNR] curve, i.e. $3881 \mathrm{mM}^{-1} \mathrm{~s}^{-1}$ (Figure 5.14, Appendix 5.2). This observation reinforces the hypothesis of the formation of highly dense paramagnetic networks in which mostly the $\mathrm{Gd}^{3+}$-SUDDO3A molecules attached to AuNRs contribute to the relaxivity enhancement observed.

\subsection{Conclusions}

In conclusion, MMAuNRs for magnetic resonance and fluorescence imaging have successfully been synthesized by simultaneous thiol-based ligand-exchange using three different moieties for 
biocompatibility/colloidal stability (PEG-SH) and $\mathrm{MRI}\left(\mathrm{Gd}^{3+}-\right.$ SUDDO3A)/optical (Biotin-SH) detection. This one-pot synthesis, using optimum ratios of $\mathrm{Gd}^{3+}-\mathrm{SUDDO} 3 \mathrm{~A}, \mathrm{PEG}-\mathrm{SH}$ and Biotin-SH (+ Streptavidin Cy5), yielded biocompatible MMAuNRs with high $\mathrm{Gd}^{3+}$ loadings. The MMAuNRs were visualized in solution by MR imaging and in vitro by fluorescence microscopy, respectively. Cell viability studies in (mouse myoblast) $\mathrm{C} 2 \mathrm{C} 12$ cells showed median lethal concentration $\left(\mathrm{LC}_{50}\right)$ values for these MMAuNRs higher than $150 \mathrm{pM}$, comparable to only PEGylated AuNRs. ${ }^{28}$ Furthermore, MMAuNRs showed a two-fold increased ionic $r_{1}$ relaxivity, thereby suggesting the formation of densely packed "paramagnetic networks" around these nanostructures as a result of entrapment of $\mathrm{Gd}^{3+}-\mathrm{SUDDO} A$ in the long PEG-SH (MW 5000) chains. Overall, these results show that AuNRs are excellent platforms for merging several imaging modalities. Moreover, MMAuNRs can be used for other imaging modalities, as AuNRs are known to exhibit intrinsic imaging behavior, e.g., for photoacoustic ${ }^{22}$ and $\mathrm{CT}^{24}$ imaging. The already known therapeutic properties of AuNRs ${ }^{20,21}$ reassure their versatility and potential as agents for theranostics.

\subsection{Experimental}

Materials. Commercial grade reagents were purchased from Sigma Aldrich and $\mathrm{TCl}$ Europe and were used without further purification unless otherwise stated. Solvents were dried over molecular sieves. Deuterated solvents were purchased from Cambridge Isotope Laboratories and NMR and MRI tubes from Wilmad LabGlass. All AuNRs were synthesized using 18.2 $\mathrm{M} \Omega / \mathrm{cm}^{2}$ water. Streptavidin-Cy5 from was purchased from Anova. O- 
[2-(3-mercaptopropionylamino)ethyl]-O'-methylpolyethylene

glycol, $\mathrm{MW}=5000$ (PEG-SH) was purchased from Aldrich.

11-Bromo-1-undecyltetrahydropyranyl sulfide (Z). This compound was synthesized following an adapted procedure from literature. ${ }^{40} 11$ bromo-1-undecanethiol (1) (250 mg, $0.94 \mathrm{mmol}$ ), dihydropyran (85 $\mu \mathrm{L}$, $0.94 \mathrm{mmol}$ ) and pyridinium $p$-toluene sulfonate $(23.5 \mathrm{mg}, 0.094 \mathrm{mmol})$ were dissolved in $25 \mathrm{~mL}$ dry $\mathrm{CH}_{2} \mathrm{Cl}_{2}$ and the reaction mixture was stirred at room temperature overnight. The solution was washed with $10 \% \mathrm{NaOH}$ $(3 \times 20 \mathrm{~mL})$, water $(3 \times 20 \mathrm{~mL})$, and dried over $\mathrm{MgSO}_{4}$. The solvent was removed under reduced pressure and the desired compound was obtained as a colorless liquid after chromatography on silica gel 60 (pretreated with $0.1 \%$ TEA in hexane) using toluene as eluent ( $\mathrm{rf} \sim 0.5$ ). Yield: 43\% (143 mg, $0.41 \mathrm{mmol}) .{ }^{1} \mathrm{H}$ NMR (600 MHz, $\left.\mathrm{CDCl}_{3}, 25^{\circ} \mathrm{C}, \mathrm{TMS}\right) \delta(\mathrm{ppm})$ : $4.86(\mathrm{~m}, 1 \mathrm{H} ; \mathrm{SCHO}), 4.1 \& 3.5\left(\mathrm{~m}, 2 \mathrm{H} ; \mathrm{OCH}_{2}\right), 3.43[\mathrm{t}, \mathrm{J}(\mathrm{H}, \mathrm{H})=6.87 \mathrm{~Hz}, 2 \mathrm{H}$; $\left.\mathrm{CH}_{2} \mathrm{Br}\right], 2.66$ \& $2.6\left(\mathrm{~m}, 2 \mathrm{H} ; \mathrm{CH}_{2} \mathrm{~S}\right), 1.95$ \& $1.69\left(\mathrm{~m}, 2 \mathrm{H} ; \mathrm{SCH}-\mathrm{CH}_{2}\right), 1.86(\mathrm{~m}$, $\left.2 \mathrm{H} ; \mathrm{CH}_{2} \mathrm{Br}-\mathrm{CH}_{2}\right), 1.83 \& 1.58\left(\mathrm{~m}, 2 \mathrm{H} ; \mathrm{SCH}-\mathrm{CH}_{2} \mathrm{CH}_{2}\right), 1.6 \& 1.59(\mathrm{~m}, 2 \mathrm{H}$; $\left.\mathrm{OCH}_{2}-\mathrm{CH}_{2}\right), 1.61\left(\mathrm{~m}, 2 \mathrm{H}, \mathrm{CH}_{2} \mathrm{~S}-\mathrm{CH}_{2}\right), 1.44\left(\mathrm{~m}, 2 \mathrm{H} ; \mathrm{CH}_{2} \mathrm{Br}_{-} \mathrm{CH}_{2}-\mathrm{CH}_{2}\right), 1.39$ $\left(\mathrm{m}, 2 \mathrm{H}, \mathrm{CH}_{2} \mathrm{~S}-\mathrm{CH}_{2}-\mathrm{CH}_{2}\right), 1.29\left(\mathrm{~m}, 10 \mathrm{H}, \mathrm{CH}_{2}\right) .{ }^{13} \mathrm{C} \mathrm{NMR}\left(150 \mathrm{MHz}, \mathrm{CDCl}_{3}\right.$, $25^{\circ} \mathrm{C}$, TMS) $\delta$ (ppm): $82.3(\mathrm{SCHO}), 64.7\left(\mathrm{OCH}_{2}\right), 34.1\left(\mathrm{CH}_{2} \mathrm{Br}\right), 32.9\left(\mathrm{CH}_{2} \mathrm{Br}-\right.$ $\left.\mathrm{CH}_{2}\right), 31.5\left(\mathrm{CH}_{2} \mathrm{~S}-\mathrm{CH}_{2}\right), 30.4\left(\mathrm{SCH}-\mathrm{CH}_{2}\right), 30\left(\mathrm{CH}_{2} \mathrm{~S}\right), 29.5$ 29.4, 29.2, 29, $28.8\left(\mathrm{CH}_{2}\right), 28.2\left(\mathrm{CH}_{2} \mathrm{Br}-\mathrm{CH}_{2}-\mathrm{CH}_{2}\right), 25.6\left(\mathrm{OCH}_{2}-\mathrm{CH}_{2}\right), 21.8\left(\mathrm{SCH}-\mathrm{CH}_{2} \mathrm{CH}_{2}\right)$. MS (ESI+, $\left.\mathrm{CH}_{2} \mathrm{Cl}_{2} / \mathrm{MeOH}\right): \mathrm{m} / \mathrm{z}: 351.3,353.3\left[\mathrm{M}^{+}+\mathrm{H}\right]$.

1,4,7,10-Tetraaza-1-(11-undecyltetrahydropyranyl

sulfide)4,7,10-triacetic acid tert-butyl ester cyclododecane (4). In a threeneck round-bottom flask, $\mathrm{K}_{2} \mathrm{CO}_{3}(483 \mathrm{mg}, 3.5 \mathrm{mmol})$ was added to a stirring solution of 1,4,7-tris(tert-butoxycarboxymethyl)-1,4,7,10- 
tetraazacyclododecane 3 (DO3A, synthesized as previously described in literature $\left.{ }^{41}\right)(600 \mathrm{mg}, 1.12 \mathrm{mmol})$ in $70 \mathrm{~mL}$ dry acetonitrile. The mixture was kept under $\mathrm{N}_{2}$ for 10 minutes under vigorous stirring. Afterwards, 11bromo-1-undecyltetrahydropyranyl sulfide (2) $(410 \mathrm{mg}, 1.12 \mathrm{mmol})$ dissolved in $15 \mathrm{~mL}$ dry $\mathrm{CH}_{2} \mathrm{Cl}_{2}$ was added slowly to the reaction mixture which was, subsequently, allowed to react for 3 days at $50^{\circ} \mathrm{C}$. The mixture was left to cool down, filtered to remove inorganic salts and the solvent was evaporated under reduced pressure. The desired compound was obtained as a yellow oil after chromatography on silica gel 60 (pre-treated with $0.1 \%$ TEA in hexane) using $\mathrm{CH}_{2} \mathrm{Cl}_{2} / \mathrm{MeOH}$ 9:1 as eluent ( $\mathrm{rf} \sim 0.5$ ). Yield: $57 \%$ (525 mg, $0.67 \mathrm{mmol}) .{ }^{1} \mathrm{H}$ NMR $\left(600 \mathrm{MHz}, \mathrm{CDCl}_{3}, 25^{\circ} \mathrm{C}, \mathrm{TMS}\right.$ ) $\delta$ (ppm): $4.83(\mathrm{~m}, 1 \mathrm{H} ; \mathrm{SCHO}), 4.08 \& 3.5\left(\mathrm{~m}, 2 \mathrm{H} ; \mathrm{OCH}_{2}\right), 3.48-2.1[\mathrm{~m}, 24 \mathrm{H}$; $\mathrm{NCH}_{2}$ (cyclen core), $\mathrm{NCH}_{2}$ (4th arm), $\mathrm{CH}_{2}-\mathrm{COOtBu}$ ), $2.63 \& 2.58(\mathrm{~m}, 2 \mathrm{H}$; $\left.\mathrm{CH}_{2} \mathrm{~S}\right), 1.92 \& 1.66\left(\mathrm{~m}, 2 \mathrm{H} ; \mathrm{SCH}-\mathrm{CH}_{2}\right), 1.83 \& 1.59\left(\mathrm{~m}, 2 \mathrm{H} ; \mathrm{SCH}-\mathrm{CH}_{2} \mathrm{CH}_{2}\right)$, $1.59 \& 1.58\left(\mathrm{~m}, 2 \mathrm{H} ; \mathrm{OCH}_{2}-\mathrm{CH}_{2}\right), 1.59\left(\mathrm{~m}, 2 \mathrm{H}, \mathrm{CH}_{2} \mathrm{~S}-\mathrm{CH}_{2}\right), 1.6[\mathrm{~m}, 6 \mathrm{H}$; $\mathrm{OCH}_{2}-\mathrm{CH}_{2}, \mathrm{CH}_{2} \mathrm{~S}-\mathrm{CH}_{2}, \mathrm{NCH}_{2}-\mathrm{CH}_{2}$ (4th arm)], 1.5-1.45 (s, 27H; $\left.\mathrm{C}\left(\mathrm{CH}_{3}\right)_{3}\right)$, 1.39 [m, 4H; $\mathrm{NCH}_{2}-\mathrm{CH}_{2}-\mathrm{CH}_{2}$ (4th arm), $\mathrm{CH}_{2} \mathrm{~S}-\mathrm{CH}_{2}-\mathrm{CH}_{2}$ ], $1.22\left(\mathrm{~m}, 10 \mathrm{H}, \mathrm{CH}_{2}\right)$. ${ }^{13} \mathrm{C}$ NMR (150 MHz, $\left.\mathrm{CDCl}_{3}, 25^{\circ} \mathrm{C}, \mathrm{TMS}\right) \delta$ (ppm): 173.5, 172.8 (COOtBu), $82.4\left[\mathrm{C}\left(\mathrm{CH}_{3}\right)_{3}\right], 82.2(\mathrm{SCHO}), 64.8\left(\mathrm{OCH}_{2}\right), 55.8,54.4,53.4,53.1,52.1,49.8$, $45.6\left[\mathrm{NCH}_{2}\right.$ (all), $\left.\mathrm{NCH}_{2}-\mathrm{CH}_{2}, \mathrm{CH}_{2} \mathrm{COOtBu}\right], 31.5\left(\mathrm{CH}_{2} \mathrm{~S}-\mathrm{CH}_{2}\right), 30.4(\mathrm{SCH}-$ $\left.\mathrm{CH}_{2}\right), 29.9\left(\mathrm{CH}_{2} \mathrm{~S}\right), 29.7,29.5,29.3,29,28.2,28.1,27.6\left(\mathrm{CH}_{2}\right), 27.8,28$ [C $\left.\left(\mathrm{CH}_{3}\right)_{3}\right], 25.6\left(\mathrm{OCH}_{2}-\mathrm{CH}_{2}\right), 21.9\left(\mathrm{SCH}-\mathrm{CH}_{2} \mathrm{CH}_{2}\right) . \mathrm{MS}\left(\mathrm{ESI}+, \mathrm{CH}_{2} \mathrm{Cl}_{2} / \mathrm{MeOH}\right)$ : $m / z: 809.2\left[\mathrm{M}^{+}+\mathrm{Na}\right]$.

\section{1,4,7,10-Tetraaza-1-(11-sulfanylundecyl)-4,7,10-triacetic acid} cyclododecane (SUDDO3A, 5). In a round-bottom flask, TFA (5 mL)

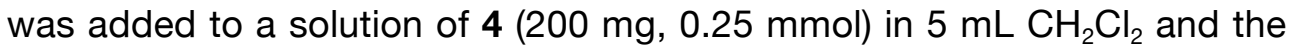
reaction mixture was allowed to stir at room temperature overnight. The 
mixture was evaporated under reduced pressure and the residue coevaporated with $\mathrm{CH}_{2} \mathrm{Cl}_{2} 3$ times in order to remove excess TFA. 5 was obtained as a solid after precipitation from diethyl ether and was subsequently used for the next reaction. Yield: quantitative. MS (MALDI+, DHB): $m / z: 533.5\left[\mathrm{M}^{+}+\mathrm{H}\right], 1066.8\left[2 \mathrm{M}^{+}+\mathrm{H}\right]$.

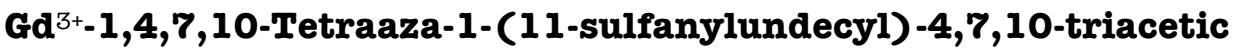
acid cycloddodecane (Gd ${ }^{3+}$-SUDDO3A, 6). In a round-bottom flask, 5 (120 mg, $0.23 \mathrm{mmol}$ ) and $\mathrm{GdCl}_{3}(92 \mathrm{mg} .0 .025 \mathrm{mmol})$ were dissolved in 50 $\mathrm{mL}$ water. The $\mathrm{pH}$ of the mixture was adjusted to 6 using $\mathrm{NaOH}(1 \mathrm{M})$ and the reaction mixture was allowed to stir at $60^{\circ} \mathrm{C}$ overnight. The solution was allowed to cool for 30 minutes and subsequently stirred for 10 minutes with (acetone washed) DOWEX Monosphere 650C cation exchange resin. After filtering in order to remove the resin, the absence of free $\mathrm{Gd}^{3+}$ ions was confirmed by the xylenol orange test. Compound 6 was obtained after the solvent was removed by lyophilization. Yield: 99\% (156 $\mathrm{mg}, 0.23 \mathrm{mmol})$. MS (ESI+, $\left.\mathrm{H}_{2} \mathrm{O} / \mathrm{ACN}+0.1 \% \mathrm{TFA}\right): \mathrm{m} / \mathrm{z}: 687.3\left[2 \mathrm{M}^{2+}+\mathrm{H}\right]$, $1218\left[2 \mathrm{M}^{+}-1 \mathrm{Gd}+\mathrm{H}\right], 1372.8\left[2 \mathrm{M}^{+}+\mathrm{H}\right]$.

(3aS,4S,6aR)-11-Bromoundecyl

5-(Hexahydro-2-oxo-1Hthieno [3,4-d] imidazol-4-yl) pentanoate (7). This compound was synthesized following an adapted procedure. ${ }^{33} \mathrm{~A}$ mixture biotin $(1.00 \mathrm{~g}$, $4.09 \mathrm{mmol})$, 11-bromo-1-undecanol (5.15 g, $20.50 \mathrm{mmol}), \quad \mathrm{p}-$ toluenesulfonic acid $(76.9 \mathrm{mg}, 0.4 \mathrm{mmol})$ and toluene $(34 \mathrm{~mL})$ was stirred and refluxed at $120{ }^{\circ} \mathrm{C}$ for $72 \mathrm{~h}$ under argon. The precipitated unreacted biotin was immediately separated by filtration and the solvent was removed in vacuo. The residue was purified by flash chromatography $\left(\mathrm{CH}_{2} \mathrm{Cl}_{2} / \mathrm{MeOH} 95: 5\right)$ to give 7 as a white solid. Yield: $68 \%$ (1.33 g, 
$2.78 \mathrm{mmol})$. MS (ESI+, $\left.\mathrm{CHCl}_{3} / \mathrm{MeOH}\right): \mathrm{m} / \mathrm{z}: 477.35\left[\mathrm{M}^{+}+\mathrm{H}\right] .{ }^{1} \mathrm{NMR}$ data were obtained in agreement with the reference. ${ }^{33}$

(3as,4S,6aR)-11-Sulfanylundecyl

5-(Hexahydro-2-oxo-1Hthieno [3,4-d]imidazol-4-yl)pentanoate (biotin-SH, 8). A stirred solution 7 (1.33 g, $2.78 \mathrm{mmol})$ in THF $(100 \mathrm{~mL})$ was cooled down to $-10^{\circ} \mathrm{C}$. Subsequently, hexamethyldisilathiane $(2.92 \mathrm{~mL}, 13.95 \mathrm{mmol})$ and TBAF (1.0 M solution in THF with $5 \%$ water, $3.29 \mathrm{~mL}, 13.95 \mathrm{mmol}$ ) were added. The reaction mixture was allowed to reach room temperature while stirring under argon and under protection from ambient light in order to avoid photoinduced side reactions. After $20 \mathrm{~min}$, the reaction mixture was washed with $20 \mathrm{~mL}$ of aqueous ammonium chloride (saturated) and with of $\mathrm{CH}_{2} \mathrm{Cl}_{2}(2 \times 10 \mathrm{~mL})$. Recrystallization from $\mathrm{CH}_{2} \mathrm{Cl}_{2}$ and hexane gave the desired compound 8 as a white solid. Yield: $89 \%$ (1.07 g, $2.48 \mathrm{mmol})$. MS $\left(\mathrm{ESI}+, \mathrm{CHCl}_{3} / \mathrm{MeOH}\right): \mathrm{m} / \mathrm{z}: 430.3\left[\mathrm{M}^{+}+\mathrm{H}\right] .{ }^{1} \mathrm{NMR}$ data were obtained in agreement with the reference. ${ }^{33}$

Nuclear Magnetic Resonance (NMR) Characterization. All NMR experiments were performed on a Bruker Avance II NMR spectrometer operating at $600.16 \mathrm{MHz}$ for ${ }^{1} \mathrm{H}$ and $150.91 \mathrm{MHz}$ for ${ }^{13} \mathrm{C}$. Chemicals shifts are given in ppm using the residual solvent signal as reference. The multiplicity of the peaks is reported using the following abbreviations: $\mathrm{s}=$ singlet, $\mathrm{d}=$ doublet, $\mathrm{t}=$ triplet, $\mathrm{q}=$ quartet, $\mathrm{m}=$ multiplet. All NMR spectra were processed using TOPSPIN 2.1 and MestReC software packages.

Mass Spectrometry (IMS). Mass spectra were acquired on a Waters Micromass LCT ESI or a Voyager-DE RP MALDI-TOF mass spectrometer. 
UV-Vis Spectroscopy. UV spectra were acquired on a Perkin Elmer Lambda 850 UV-Vis spectrometer using Plastibrand $1.5 \mathrm{~mL}$ semi-micro cuvettes.

Fluorescence Spectroscopy. All steady-state spectra were recorded using a Perkin Elmer Fluorescence Spectrometer and 1cm quartz cuvettes.

Magnetic Resonance Imaging (IMRI). All experiments were carried out on a $600 \mathrm{MHz}$ (14.1) Avance II NMR Spectrometer from Bruker (Karlsruhe, Germany) equipped with a vertical narrow bore magnet (14.1 $T$ ), a great $B_{0}$ compensation unit (BGU-II) and 3 great $1 / 60$ amplifier units $(\mathrm{X}, \mathrm{Y}$ and $\mathrm{Z}$ ) also from Bruker. A micro 5 imaging probe, equipped with a $10 \mathrm{~mm}$ diameter saddle coil insert from Bruker (Karlsruhe, Germany) was used. The maximum gradient strength was $4.8 \mathrm{G} / \mathrm{cm} / \mathrm{A}$ and the temperature inside the probe was kept at $\sim 25^{\circ} \mathrm{C}$ by a gradient water cooling unit BCU20 from Bruker. All NMR/MRI tubes (5/10 mm diameter) were purchased from Wilmad Labglass (New Jersey, U.S.A.). Glass capillary tubes (melting point tubes, inner diameter $1 \mathrm{~mm}$ ) used for MRI were purchased from VWR. $T_{1}$ measurements were performed in a $1 \mathrm{~cm}$ (outer diameter) glass tube as follows. Stock solutions of the $\mathrm{Gd}^{3+}$ SUDDO3A complex and the MMAuNRs (with known concentrations of $\mathrm{Gd}^{3+}$ and AuNRs) were diluted 2, 5 and 10 times. For each experiment, capillary tubes of $1 \mathrm{~mm}$ inner diameter were filled with these solutions, placed in a custom-made Kel-F capillary holder and subsequently introduced in the $1 \mathrm{~cm}$ tube for imaging. Longitudinal relaxation times were measured using a saturation recovery pulse sequence with static $\mathrm{TE}=4 \mathrm{~ms}$ and $16 \mathrm{TR}$ values, namely $100,150,200,350,500,600,700$, $800,1000,1500,2000,3000,4000,6000,8000,10000 \mathrm{~ms}$. The 
measurements were done on 5 subsequent axial slices of $1 \mathrm{~mm}$, with a field of view (FOV) of $10 \mathrm{~mm} \times 10 \mathrm{~mm}$, a matrix size of $128 \times 128$ and 2 averages. $T_{1}$ values were obtained by mono-exponentially fitting the intensities of selected regions of interest (ROls) for each axial slice using the image sequence analysis tool in Paravision 4.0 software. $T_{1}$-weighted images were obtained using a spin echo sequence with the following parameters: TR $=500 \mathrm{~ms}, \mathrm{TE}=4 \mathrm{~ms}, 1$ axial slice of $6 \mathrm{~mm}$ thickness, FOV $=10 \mathrm{~mm} \times 10 \mathrm{~mm}$, matrix size $=256 \times 256$ and 10 averages. The longitudinal relaxivity $r_{1}$ (in $\mathrm{mM}^{-1} \mathrm{~s}^{-1}$ ) was obtained as the slope of the plot $1 / T_{1}$ vs. $\mathrm{Gd}^{3+}$ (or AuNR) concentration:

$$
\left(\frac{1}{T_{1}}\right)_{o b s}=\left(\frac{1}{T_{1}}\right)_{d}+r_{1}[G d]
$$

\section{Transmission Flectron Microscopy (TEM) and Fnergy-Dispersive} X-Ray (EDX). TEM images were obtained using analytical TEM FEI instruments. In order to prepare the samples, $10 \mu \mathrm{L}$ of the desired sample was applied onto Formvar-carbon coated grids (from electron microscopy sciences). After leaving the sample for 1 minute, the excess of liquid was removed using a piece of filter paper. The average aspect ratio (length/width) of the different AuNRs was determined by measuring their length and width, for more than 50 counts, from TEM images.

Cell Culture. C2C12 (Mouse myoblast) cells were plated in a 96 well plate at a density of 5000 cells/well in Dulbecco's modified eagle medium (DMEM) media (Sigma-Aldrich). The medium was supplemented with 4.5 g/L glucose, L-glutamine and $\mathrm{NaHCO}_{3} ; 1 \%$ (v/v) Penicillin-Streptomycin (with 10,000 units penicillin and $10 \mathrm{mg}$ streptomycin/mL, Sigma-Aldrich). The cells were cultured at $37^{\circ} \mathrm{C}$ in a humidified $5 \% \mathrm{CO}_{2}$ atmosphere for 16 
hours. Subsequent to equilibration, adhered cells were carefully washed twice with sterile PBS buffer to remove loosely attached cells.

Cell Viability Assay. Cell viability as a response to AuNRs (supplemented in the culture media) was studied using the CellTiter $96 \AA$ AQueous One Solution Cell Proliferation Assay Test (Promega, The Netherlands). After removing loosely attached cells, the test AuNRs were supplemented $(50 \mu \mathrm{L})$ in fresh culture media in four or five serial dilutions (in triplicate, see Table 5.5). The same series of dilutions were added without any cells (in duplicate) to be used as sample background (SB) control. Culture medium without the test compounds and cells was used as a medium-only control ( $\left.\mathrm{LC}_{0}\right)$ and $\mathrm{C} 2 \mathrm{C} 12$ cells were used at the same density as cell-only control ( $\left(\mathrm{LC}_{100}\right)$. Cells were exposed to the AuNRs for 24 $\mathrm{h}$ in the same culture conditions described above. Subsequently, $20 \mu \mathrm{L}$ of CellTiter 96® AQueous One Solution Reagent ([3-(4,5-dimethylthiazol-2yl)-5-(3-carboxymethoxyphenyl)-2-(4-sulfophenyl)-2H-tetrazolium, inner salt (MTS) and an electron coupling reagent (phenazine ethosulfate, PES) were added to all wells. The plates were shielded from ambient light and incubated for another $4 \mathrm{~h}$ at the culture conditions. The absorbance was then recorded at $490 \mathrm{~nm}$ using a Tecan plate reader. A reference wavelength of $630 \mathrm{~nm}$ was used to subtract the background contributed by excess cell debris, fingerprints and other nonspecific absorbance. 
Table 5.5 AuNRs concentrations used for cell viability studies

\begin{tabular}{|c|c|c|c|c|c|c|}
\hline Incubation conditions & 0 & 10 & 50 & 100 & 150 & 200 \\
\hline $100 \%$ PEG-SH & $\mathbf{x}$ & $\mathbf{x}$ & $\mathbf{x}$ & $\mathbf{x}$ & & $\mathbf{x}$ \\
\hline $75 \%$ PEG-SH / 25\% Gd GU $^{3+}$ SUDD3A & $\mathbf{x}$ & $\mathbf{x}$ & $\mathbf{x}$ & $\mathbf{x}$ & & $\mathbf{x}$ \\
\hline $50 \%$ PEG-SH / 50\% Gd $^{3+}$-SUDDO3A & $\mathbf{x}$ & $\mathbf{x}$ & $\mathbf{x}$ & $\mathbf{x}$ & & $\mathbf{x}$ \\
\hline 25\% PEG-SH / 75\% Gd G $^{3+}$-SUDDO3A & $\mathbf{x}$ & $\mathbf{x}$ & $\mathbf{x}$ & $\mathbf{x}$ & & $\mathbf{x}$ \\
\hline $100 \% \mathrm{Gd}^{3+}$-SUDDO3A & $\mathbf{x}$ & $\mathbf{x}$ & $\mathbf{x}$ & $\mathbf{x}$ & & $\mathbf{x}$ \\
\hline $50 \%$ PEG-SH / 50\% Biotin-SH* & $\mathbf{x}$ & $\mathbf{x}$ & $\mathbf{x}$ & $\mathbf{x}$ & $\mathbf{x}$ & \\
\hline $\begin{array}{c}25 \% \text { PEG-SH /25\% Gd }{ }^{3+}-S U D D O 3 A \\
\text { Biotin-SH* }\end{array}$ & $\mathbf{x}$ & $\mathbf{x}$ & $\mathbf{x}$ & $\mathbf{x}$ & $\mathbf{x}$ & \\
\hline
\end{tabular}

* These AuNRs were incubated with Streptavidin-Cy5 before use

Data processing and analysis was done following an adapted procedure from literature. ${ }^{28}$ Briefly, the average values of the absorbance readings for the triplicates were calculated and corrected by deducting the average absorbance from the sample background control for each case. The standard deviation of the corrected values $(\sigma)$ was calculated from the linear combination of errors.

The corrected absorbance for the cell-only control was calculated by subtracting the average absorbance of the medium-only control. This is taken as the absorbance associated with living cells. The percentage of surviving cells is then calculated dividing the corrected sample absorbance by the corrected cell-only absorbance and multiplied by 100 . The corresponding standard deviation was calculated following the quotient combination of errors.

Cell Staining. After removal of the cell culture medium, cells were washed with sterile PBS once and subsequently fixed with a $4 \%$ formaldehyde solution in PBS for 10 minutes at room temperature. Afterwards, C2C12 cells were stained for F-actin with Alexa Fluor ${ }^{\circledR} 488$ 
phalloidin (Invitrogen) and DAPI, dilactate (Invitrogen) following the protocol from the provider. Finally, the substrates were washed two times with PBS, water and were carefully blown-dry with $\mathrm{N}_{2}$.

Fluorescence Microscopy. Fluorescence microscopy images were taken using an Olympus inverted research microscope IX71 equipped with a mercury burner U-RFL-T as light source and a digital Olympus DR70 camera for image acquisition using the following Olympus filter cubes: 300 $\mathrm{nm} \leq \lambda_{\mathrm{ex}} \leq 400 \mathrm{~nm}$ for blue emission (410 nm $\left.\leq \lambda_{\mathrm{em}} \leq 510 \mathrm{~nm}\right), 460 \mathrm{~nm} \leq \lambda_{\mathrm{ex}}$ $\leq 490 \mathrm{~nm}$ for green emission $\left(\lambda_{\mathrm{em}} \geq 520\right)$ and $630 \mathrm{~nm} \leq \lambda_{\mathrm{ex}} \leq 650 \mathrm{~nm}$ for Cy5 emission (665 $\mathrm{nm} \leq \lambda_{\mathrm{ex}} \leq 695 \mathrm{~nm}$ ). All fluorescence microscopy images were acquired in air.

Total Reflection X-ray Fluorescence (TXRF). TXRF measurements were performed by a Bruker S2 Picofox TXRF (Bruker AXS GmbH, Karlsruhe, Germany) portable system. The primary $X$ rays were generated by an air-cooled low power X-ray tube using a molybdenum anode. The excitation settings were $50 \mathrm{kV}$ and $750 \mathrm{~mA}$. Measurements were performed by signal integration over 1500 seconds. Quartz glass disks were used as sample carriers, which were previously cleaned in $1 \mathrm{~N}$ nitric acid followed by flushing in water for $1 \mathrm{~h}$ at $80^{\circ} \mathrm{C}$. After that, they were dried, coated with silicon solution for better wettability and dried again. The quantification is performed by the Bruker S2 Picofox TXRF software (Spectra, Version 6.1.5.0), based on the known quantity of the gallium internal standard added. Samples for TXRF analysis were prepared by adding equal amounts of the gallium internal standard $(10 \mathrm{mg} / \mathrm{L})$ and the sample to be analyzed (volume). After vortexing, a drop of the solution was placed on the quartz disk and dried under vacuum for 15 minutes. For 
control purposes, elemental analysis of the empty capsid (in buffer) was conducted and the values obtained, for each capsid sample in question, were corrected.

\subsection{References}

(1) Cassidy, P. J.; Radda, G. K. J. R. Soc. Interface 2005, 2, 133.

(2) Weissleder, R.; Mahmood, U. Radiology 2001, 219, 316.

(3) Caravan, P.; Ellison, J. J.; McMurry, T. J.; Lauffer, R. B. Chem. Rev. 1999, 99, 2293.

(4) Bottrill, M.; Kwok, L.; Long, N. J. Chem. Soc. Rev. 2006, 35, 557.

(5) Jin, T.; Yoshioka, Y.; Fujii, F.; Komai, Y.; Seki, J.; Seiyama, A. Chem. Commun. 2008, 5764.

(6) Mulder, W. J. M.; Koole, R.; Brandwijk, R. J.; Storm, G.; Chin, P. T. K.; Strijkers, G. J.; de Mello Donegá, C.; Nicolay, K.; Griffioen, A. W. Nano Lett. 2005, 6,1 .

(7) Yang, H.; Santra, S.; Walter, G. A.; Holloway, P. H. Adv. Mater. 2006, 18, 2890.

(8) Alric, C.; Taleb, J.; Duc, G. L.; Mandon, C.; Billotey, C.; Meur-Herland, A. L.; Brochard, T.; Vocanson, F.; Janier, M.; Perriat, P.; Roux, S.; Tillement, O. J. Am. Chem. Soc. 2008, 130, 5908.

(9) Debouttière, P. J.; Roux, S.; Vocanson, F.; Billotey, C.; Beuf, O.; FavreRéguillon, A.; Lin, Y.; Pellet-Rostaing, S.; Lamartine, R.; Perriat, P.; Tillement, O. Adv. Funct. Mater. 2006, 16, 2330.

(10) Moriggi, L. C.; Cannizzo, C.; Dumas, E.; Mayer, C. d. R.; Ulianov, A.; Helm, L. J. Am. Chem. Soc. 2009, 131, 10828.

(11) Park, J.-A.; Reddy, P. A. N.; Kim, H.-K.; Kim, I.-S.; Kim, G.-C.; Chang, Y.; Kim, T.-J. Bioorg. Med. Chem. Lett. 2008, 18, 6135.

(12) Song, Y.; Xu, X.; MacRenaris, K. W.; Zhang, X.-Q.; Mirkin, C. A.; Meade, T. J. Angew. Chem., Int. Ed. 2009, 48, 9143.

(13) Warsi, M. F.; Chechik, V. Phys. Chem. Chem. Phys. 2011, 13, 9812.

(14) Murphy, C. J.; Gole, A. M.; Stone, J. W.; Sisco, P. N.; Alkilany, A. M.; Goldsmith, E. C.; Baxter, S. C. Acc. Chem. Res. 2008, 41, 1721.

(15) Huang, X.; Neretina, S.; El-Sayed, M. A. Adv. Mater. 2009, 21, 4880.

(16) Pérez-Juste, J.; Pastoriza-Santos, I.; Liz-Marzán, L. M.; Mulvaney, P. Coord. Chem. Rev. 2005, 249, 1870.

(17) Tong, L.; Wei, Q.; Wei, A.; Cheng, J.-X. Photochem. Photobiol. 2009, 85, 21.

(18) Thakor, A. S.; Jokerst, J.; Zavaleta, C.; Massoud, T. F.; Gambhir, S. S. Nano Lett. 2011, 11, 4029.

(19) Giljohann, D. A.; Seferos, D. S.; Daniel, W. L.; Massich, M. D.; Patel, P. C.; Mirkin, C. A. Angew. Chem., Int. Ed. 2010, 49, 3280. 
(20) Zhou, W.; Shao, J.; Jin, Q.; Wei, Q.; Tang, J.; Ji, J. Chem. Commun. 2010, $46,1479$.

(21) Yi, D. K.; Sun, I.-C.; Ryu, J. H.; Koo, H.; Park, C. W.; Youn, I.-C.; Choi, K.; Kwon, I. C.; Kim, K.; Ahn, C.-H. Bioconjugate Chem. 2010, 21, 2173.

(22) Manohar, S.; Ungureanu, C.; Van Leeuwen, T. G. Contrast Media Mol. I. 2011, 6, 389.

(23) Liu, L.; Ding, H.; Yong, K.-T.; Roy, I.; Law, W.-C.; Kopwitthaya, A.; Kumar, R.; Erogbogbo, F.; Zhang, X.; Prasad, P. Plasmonics 2011, 6, 105.

(24) Sun, H.; Yuan, Q.; Zhang, B.; Ai, K.; Zhang, P.; Lu, L. Nanoscale 2011, 3, 1990.

(25) Cortesi, R.; Esposito, E.; Menegatti, E.; Gambari, R.; Nastruzzi, C. Int. J. Pharm. 1996, 139, 69.

(26) Takahashi, H.; Niidome, Y.; Niidome, T.; Kaneko, K.; Kawasaki, H.; Yamada, S. Langmuir 2005, 22, 2.

(27) Kopwitthaya, A.; Yong, K.-T.; Hu, R.; Roy, I.; Ding, H.; Vathy, L. A.; Bergey, E. J.; Prasad, P. N. Nanotechnology 2010, 21, 315101.

(28) Rayavarapu, R. G.; Petersen, W.; Hartsuiker, L.; Chin, P.; Janssen, H.; van Leeuwen, F. W. B.; Otto, C.; Manohar, S.; van Leeuwen, T. G. Nanotechnology 2010, 21.

(29) Vigderman, L.; Manna, P.; Zubarev, E. R. Angew. Chem., Int. Ed. 2012, 51,636 .

(30) Basiruddin, S. K.; Maity, A. R.; Saha, A.; Jana, N. R. J. Phys. Chem. C 2011, 115, 19612.

(31) Basiruddin, S. K.; Saha, A.; Pradhan, N.; Jana, N. R. Langmuir 2010, 26, 7475.

(32) Weber, P.; Ohlendorf, D.; Wendoloski, J.; Salemme, F. Science 1989, 243, 85.

(33) Reichmuth, P.; Sigrist, H.; Badertscher, M.; Morf, W. E.; de Rooij, N. F.; Pretsch, E. Bioconjugate Chem. 2001, 13, 90.

(34) Castellana, E. T.; Gamez, R. C.; Russell, D. H. J. Am. Chem. Soc. 2011, 133, 4182.

(35) Orendorff, C. J.; Murphy, C. J. J. Phys. Chem. B 2006, 110, 3990.

(36) Link, S.; El-Sayed, M. A. J. Phys. Chem. B 1999, 103, 8410.

(37) Telgmann, L.; Holtkamp, M.; Kunnemeyer, J.; Gelhard, C.; Hartmann, M.; Klose, A.; Sperling, M.; Karst, U. Metallomics 2011, 3, 1035.

(38) Kobayashi, H.; Kawamoto, S.; Bernardo, M.; Brechbiel, M. W.; Knopp, M. V.; Choyke, P. L. J. Controlled Release 2006, 111, 343.

(39) Rieter, W. J.; Kim, J. S.; Taylor, K. M. L.; An, H.; Lin, W.; Tarrant, T.; Lin, W. Angewandte Chemie, International Edition 2007, 46, 3680.

(40) Block, E.; Eswarakrishnan, V.; Gernon, M.; Ofori-Okai, G.; Saha, C.; Tang, K.; Zubieta, J. J. Am. Chem. Soc. 1989, 111, 658.

(41) Beeby, A.; Bushby, L. M.; Maffeo, D.; Gareth Williams, J. A. J. Chem. Soc., Dalton Trans. 2002, 48.

(42) Wilhelm, M.; Zhao, C. L.; Wang, Y.; Xu, R.; Winnik, M. A.; Mura, J. L.; Riess, G.; Croucher, M. D. Macromolecules 1991, 24, 1033. 


\section{Appendix 5.1. Calculation of the Critical Aggregation Concentration (cac) of $\mathrm{Gd}^{3+}-$ SUDDO3A}

The critical aggregation concentration of $\mathrm{Gd}^{3+}-\mathrm{SUDDO} 3 \mathrm{~A}$, calculated to be $0.63 \mathrm{mM}$, was obtained using a previously reported pyrene-based procedure. ${ }^{42}$ In short, a known amount of pyrene in acetone was added to nine $\mathrm{Gd}^{3+}$-SUDDO3A sample solutions (concentrations ranging from 0.0025 to $0.0001 \mathrm{M}$ in water), to give a final pyrene concentration of $6 \times 10^{-7} \mathrm{M}$ (saturation solubility in water). After heating and cooling overnight to guarantee micelle formation, the emission spectra of all nine solutions was taken $\left(\lambda_{\mathrm{exc}}=332 \mathrm{~nm}\right)$. In a plot of the fluorescence intensity at $387 \mathrm{~nm}$ vs. log $\left[\mathrm{Gd}^{3+}-\mathrm{SUDDO} 3 \mathrm{~A}\right]$ (Figure 5.12), the intersection of the two tangents (before and after the inflection point) corresponds to the critical aggregation concentration (cac).

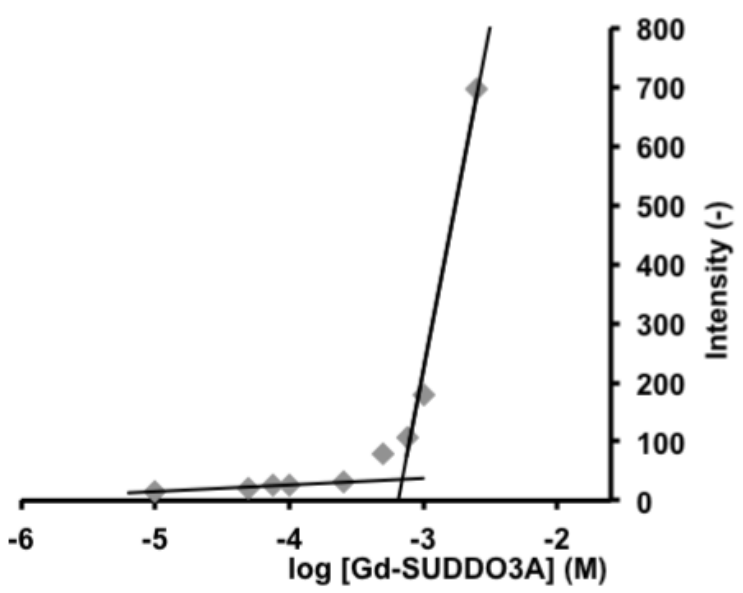

Figure 5.12. Plot of the fluorescence intensity of pyrene $\left(\lambda_{\mathrm{exc}}=332 \mathrm{~nm}\right)$ as a function of the $\mathrm{Gd}^{3+}$-SUDDO3A concentration. 


\section{Appendix 5.2. MRI Data}

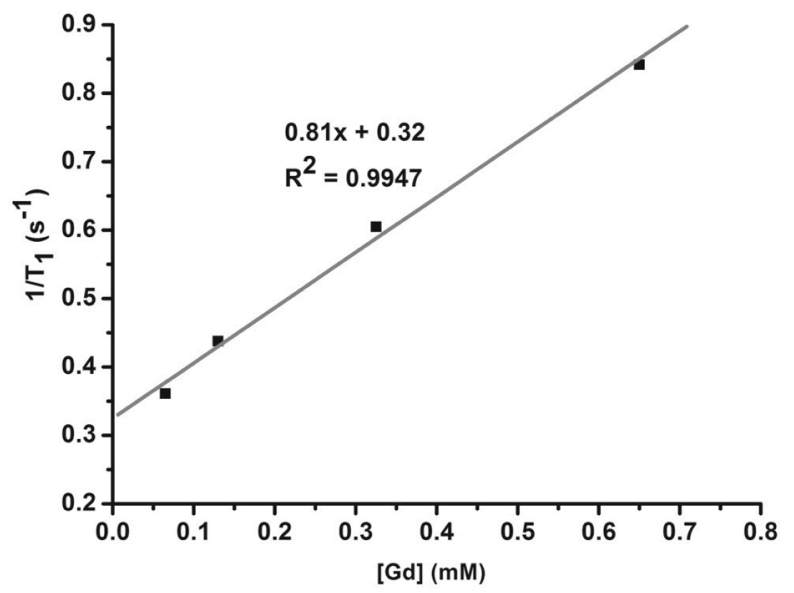

Figure 5.13. Relaxivity plot of the $\mathrm{Gd}^{3+}-\mathrm{SUDDO} 3 \mathrm{~A}$ complex at $600 \mathrm{MHz}$ and $25^{\circ} \mathrm{C}$.

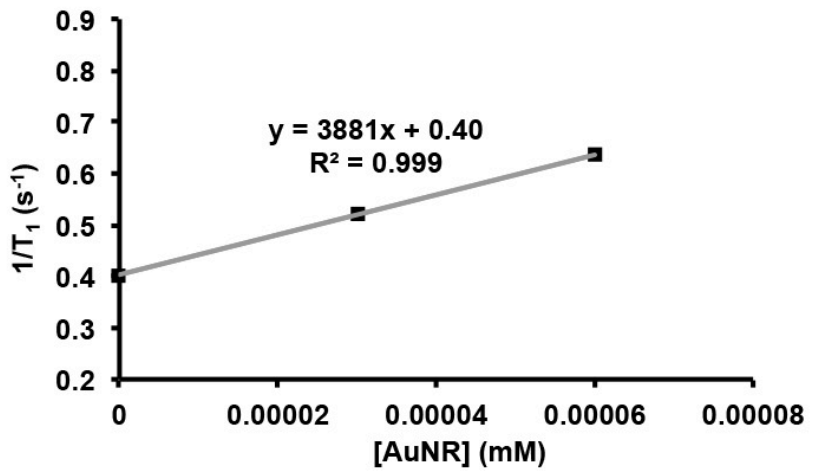

Figure 5.14. Relaxivity plot of the MMAuNRs (with respect to the AuNR concentration) at $600 \mathrm{MHz}$ and $25^{\circ} \mathrm{C}$ 
Table 5.6. $T_{1}$ values obtained for the $\mathrm{Gd}^{3+}-\mathrm{SUDDO} 3 \mathrm{~A}$ complex at $600 \mathrm{MHz}$ and $25^{\circ} \mathrm{C}$

\begin{tabular}{|c|c|c|c|c|c|c|c|c|}
\hline $\begin{array}{c}{\left[\mathrm{Gd}^{3+}\right]} \\
\mathbf{m M}\end{array}$ & $\begin{array}{c}T_{1} \\
\text { Slice 1 } \\
(\mathbf{m s})\end{array}$ & $\begin{array}{c}T_{1} \\
\text { Slice 2 } \\
(\mathbf{m s})\end{array}$ & $\begin{array}{c}T_{1} \\
\text { Slice 3 } \\
(\mathbf{m s})\end{array}$ & $\begin{array}{c}T_{1} \\
\text { Slice 4 } \\
(\mathbf{m s})\end{array}$ & $\begin{array}{c}T_{1} \\
\text { Slice 5 } \\
(\mathbf{m s})\end{array}$ & $\begin{array}{c}\text { Average } \\
(\mathbf{m s})\end{array}$ & $\begin{array}{c}\text { Stand. } \\
\text { Dev. } \\
(\mathbf{m s})\end{array}$ & $\begin{array}{c}1 / T_{1} \\
\left(\mathbf{s}^{-1}\right)\end{array}$ \\
\hline 0.65 & 1153.2 & 1206.9 & 1198 & 1202.3 & 1177.5 & 1187.6 & 22.2 & 0.842 \\
\hline 0.325 & 1594.3 & 1695.6 & 1659.8 & 1686 & 1625.9 & 1652.3 & 42.2 & 0.605 \\
\hline 0.130 & 2193.7 & 2298.7 & 2336.3 & 2327.2 & 2268.7 & 2284.9 & 57.5 & 0.438 \\
\hline 0.065 & 2732.7 & 2793.2 & 2798.5 & 2777.2 & 2760.3 & 2772.4 & 26.7 & 0.361 \\
\hline
\end{tabular}

Table 5.7. $T_{1}$ values obtained for the MMAuNRs at $600 \mathrm{MHz}$ and $25^{\circ} \mathrm{C}$

\begin{tabular}{|c|c|c|c|c|c|c|c|c|}
\hline $\begin{array}{c}{\left[\mathrm{Gd}^{3+}\right]} \\
\mathrm{mM}\end{array}$ & $\begin{array}{c}T_{1} \\
\text { Slice } 1 \\
\text { (ms) }\end{array}$ & $\begin{array}{c}T_{1} \\
\text { Slice } 2 \\
\text { (ms) }\end{array}$ & $\begin{array}{c}T_{1} \\
\text { Slice } 3 \\
\text { (ms) }\end{array}$ & $\begin{array}{c}T_{1} \text { Slice } \\
4 \text { (ms) }\end{array}$ & $\begin{array}{c}T_{1} \\
\text { Slice } 5 \\
\text { (ms) }\end{array}$ & $\begin{array}{c}\text { Average } \\
\text { (ms) }\end{array}$ & $\begin{array}{l}\text { Stand. } \\
\text { Dev. } \\
\text { (ms) }\end{array}$ & $\begin{array}{l}1 / T_{1} \\
\left(s^{-1}\right)\end{array}$ \\
\hline 0.643 & 840.5 & 943.5 & 914.4 & 915.7 & 909.3 & 904.7 & 38.3 & 1.105 \\
\hline 0.129 & 1570.2 & 1628.7 & 1604.5 & 1548 & 1518.2 & 1573.9 & 43.9 & 0.635 \\
\hline 0.064 & 1797.2 & 1930.3 & 1938.3 & 1981.9 & 1937.7 & 1917.1 & 70.0 & 0.522 \\
\hline 0 & 2372.8 & 2484.6 & 2534.9 & 2556.6 & 2472.4 & 2484.3 & 71.3 & 0.403 \\
\hline
\end{tabular}


CHAPTER 5 
"Et il est un jour arrive / Marteler le ciel/ Et marteler la mer Et la mer avait embrassé moil Et la délivré moi de ma caille

Rien ne peut $m$ 'arrêter maintenant" La mer, Nine Inch Nails

\section{CHAPTER 6}

\section{FUTURE PERSPECTIVES: METAL COMPLEX- BASED TEMPLATES IN CARBON-BASED SCAFFOLDS AS MAGNETIC RESONANCE/ OPTICAL MULTIMODAL IMAGING AGENTS}

\section{Abstract}

The future perspectives of the metal complex-based templates and the multimodal imaging agents described in this thesis are discussed here. The versatility of the 1,4,7,10-tetraazacyclododecane-1,4,7-triacetic acid (DO3A)based ligands described in previous chapters is demonstrated by their integration in carbon-based scaffolds such as small organic molecules and carbon nanotubes (CNTs). The preliminary characterization and evaluation of their properties presented here show promising results, thereby highlighting their potential for multimodal purposes. Finally, challenges ahead for the metal complex-based templates and magnetic resonance/optical multimodal imaging agents presented in this thesis are briefly discussed with an outlook toward the future. 


\subsection{Introduction}

Molecular imaging has developed as a diagnostic discipline dedicated to the visualization of biological processes in the human body in an, ideally but not always attainable, non-invasive manner. Throughout the years, several strategies and methodologies for the synthesis of multimodal imaging agents (IAs), needed in order to aid the visualization of region of interest (ROIs), have been followed. While many of them are based on pure synthetic chemistry, ${ }^{1-4}$ the combination of existing chromophores and metal complexes with nanomaterials is currently a highly explored approach. ${ }^{5-11}$ Inspired by the latter, the results presented in this thesis show that 1,4,7,10-tetraazacyclododecane-1,4,7-triacetic acid (DO3A)based ligands are highly suitable candidates for (multimodal) molecular imaging probes either by i) acting as templates for the growth of nanomaterials (as shown in Chapter 4), or ii) as chelates for metal complexation in nanomaterials (e.g., protein cages and gold nanorods, Chapters 5 and 6 respectively).

Lanthanides have been widely used for the fabrication of IAs as these elements show strikingly different electronic and magnetic properties. ${ }^{12}$ Whereas gadolinium $\left(\mathrm{Gd}^{3+}\right)$ is known for its excellent paramagnetic properties, ${ }^{13-15}$ europium $\left(\mathrm{Eu}^{3+}\right)$ and terbium $\left(\mathrm{Tb}^{3+}\right)$ are highly attractive owing to their fluorescent properties. ${ }^{16,17}$ In the latter cases, an antenna or sensitizer (e.g., a chromophore ${ }^{18-22}$ or a transition metal) (3-26 $^{23}$ needed for energy transfer to the lanthanide as its electric dipole $f-f$ transitions have very low extinction coefficients and are Laporte forbidden. ${ }^{16}$ Remarkably, lanthanides can be chelated by polydentate ligands in a rather comparable manner since they are, chemically, very similar. 14,16,17 The previously mentioned features have led to the development of bimodal molecular 
probes that can be used for either optical or magnetic resonance imaging (MRI). ${ }^{4,23,27-30}$

In this chapter, the use of functionalized carbon-based organic scaffolds as platforms for multiple lanthanide complexation is proposed. First, a small, water-soluble carbon-based scaffold, consisting of an anthracene core where two identical DO3A-based chelators (similar to the one presented in Chapter 5) are integrated, is presented. Preliminary results show the promising paramagnetic and optical properties of the final metal complex. Subsequently, the covalent attachment of the AB-DO3A template (shown in Chapter 4) to a bigger carbon-based scaffold, i.e. carbon nanotubes (CNTs), is debated together with some preliminary characterization. The scaffolds discussed here are envisaged as excellent candidates for multimodal molecular imaging applications. Finally, the challenges ahead are briefly discussed.

\subsection{An Anthracene-Core Multimodal Agent for Magnetic Resonance and Fluorescence Imaging}

The successful synthesis and implementation of bimodal imaging probes paved the way for the development of IAs where various imaging modalities are combined within one molecule. Initially, the first di- and trinuclear lanthanide complexes described in the literature consisted of combinations of only fluorescent lanthanides for sensing purposes. ${ }^{31,32}$ The first example of a potential di-nuclear lanthanide complex for multimodal imaging was described by Mamedov et al, ${ }^{2}$ who linked two different chelating moieties using a benzene-based antenna. The formation of bimetallic species was confirmed by means of luminescence and NMR diffusion studies. 
Here, a small, water-soluble ligand suitable for multiple lanthanide complexation is presented. The bis-macrocyclic ligand ANT-2DO3A consists of an anthracene core where two (identical) 1,4,7,10tetraazacyclododecane-1,4,7-triacetic acid (DO3A)-based chelators are integrated. Therefore, the ANT-2DO3A ligand is expected to show comparable affinity to various lanthanide ions. The characterized ligand was subsequently complexed with a $50 \% \mathrm{Gd}^{3+} / 50 \% \mathrm{Eu}^{3+}$ mixture, thereby leading to the formation of a multimodal IA, as suggested by its fluorescent and paramagnetic properties. This approach serves as a proof-of-principle for further development of small molecule, multimodal IAs by exploiting the chemically similar, but electronically/magnetically different, properties lanthanides show.

\subsubsection{Design and Synthesis}

Within this study the model ligand serving as proof-of-principle is a symmetrical bis-macrocycle, consisting of two identical chelating units for lanthanide complexation tethered by an anthracene-base core. The choice for identical chelating units is based on the hypothesis that all lanthanides are complexed by a single chelator in a similar fashion. ${ }^{14,16,17}$ Consequently, equal affinity to any lanthanide upon reaction is assumed. Moreover, the symmetrical, equally substituted anthracene core is an excellent platform for integration of chelators using the same chemistry, thereby reducing the number of synthetic steps and simplifying the characterization by NMR. More importantly, the anthracene core fulfills a second function by serving as an antenna for energy transfer to the fluorescent lanthanide.

The synthesis of the bis-macrocyclic ligand for lanthanide complexation (ANT-2DO3A) was carried out in three steps, namely: i) integration of a propyl alkylated maleimide linker to the anthracene core (Scheme 6.1), ii) 
integration of a thiolated propyl linker to the 1,4,7,10tetraazacyclododecane-1,4,7-triacetic acid (DO3A) chelator (Scheme 6.2) and iii) reaction of the DO3A-derivative with the anthracene core via a Michael-type addition reaction (Scheme 6.3).

\subsubsection{Synthesis of the Building Blocks}

The synthesis of the anthracene-core building block 9,10-bis[methyl-4- $N$ (butanamide)maleimide]anthracene (3) is shown in Scheme 6.1. The starting material, 9,10-bis(aminomethyl)anthracene (1) was synthesized by bromoalkylation of anthracene ${ }^{33}$ and subsequent nucleophilic substitution ${ }^{34}$ using adapted literature procedures. The bromopropyl linker was incorporated on both sides of (1) by the formation of an amide in chloroform using 4-bromobutyryl chloride (under $\mathrm{N}_{2}$ ) in the presence of $N, N$ '-diisopropylethylamine (DIPEA). Finally, the substituted anthracene core 3 was obtained by reacting maleimide with (2) using $\mathrm{K}_{2} \mathrm{CO}_{3}$ in acetonitrile (at $50^{\circ} \mathrm{C}$ ) for 24 hours. Compound 3 was obtained with an overall yield (after purification) of $52 \%$.

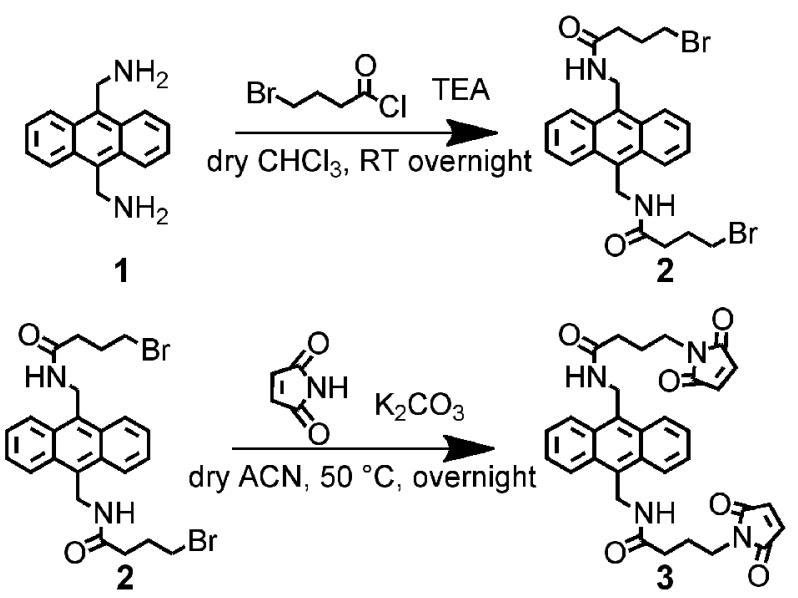

Scheme 6.1. Reaction scheme showing the synthetic pathway for the synthesis of the anthracene-core building block 9,10-bis[methyl-4- $N$-(butanamide)maleimide]anthracene (3). 
The synthesis of the second building block of the bis-macrocyclic ligand ANT-2DO3A, the thiolated 1,4,7,10-tetraazacyclododecane-1,4,7-triacetic acid (DO3A) chelator (8), is shown in Scheme 6.2. First, 3-chloro-1propanethiol (4) was protected by reaction with dihydropyran in the presence of pyridinium $p$-toluene sulfonate in $\mathrm{CH}_{2} \mathrm{Cl}_{2}$ overnight at room temperature. To obtain 7, 1,4,7-tris(tert-butoxycarboxymethyl)-1,4,7,10tetraazacyclododecane (6) was alkylated with the protected thiol (5) in dry acetonitrile using $\mathrm{K}_{2} \mathrm{CO}_{3}$ and $\mathrm{KI}$ (under $\mathrm{N}_{2}$ ) for three days at $50^{\circ} \mathrm{C}$. The chelator acid-1,4,7,10tetraazacyclododecane (8) was formed by deprotection of (7) at room temperature using TFA/ $\mathrm{CH}_{2} \mathrm{Cl}_{2}$ overnight with an overall yield (after purification) of $24 \%$.

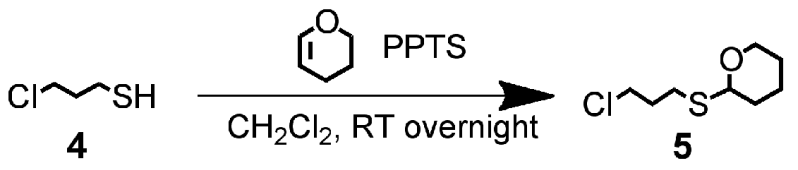

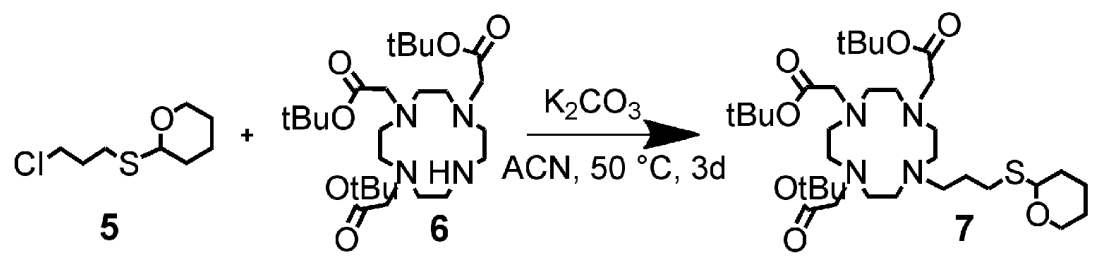

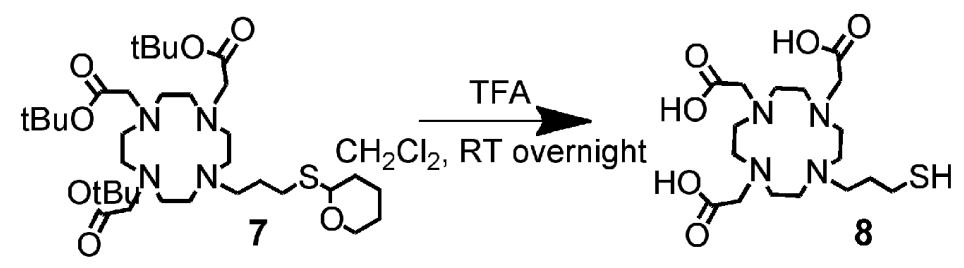

Scheme 6.2. Reaction scheme showing the synthetic pathway followed for the synthesis of 1-(3-mercaptopropyl)-4,7,10-triacetic acid-1,4,7,10-tetraazacyclododecane (8). 
6.2.3 Synthesis of the ANT-2DO3A Ligand and its Lanthanide Complexes

The building blocks $\mathbf{3}$ and $\mathbf{8}$ were allowed to react in methanol overnight (Scheme 6.3), leading to the formation of the ligand 9 via a thiol-maleimide Michael-type addition. After dialysis in water for three days, ANT-2DO3A (9) was obtained with a yield of $5 \%$.

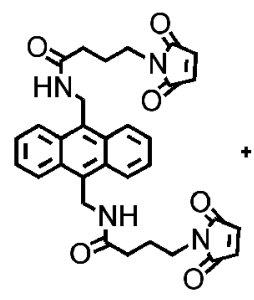

3

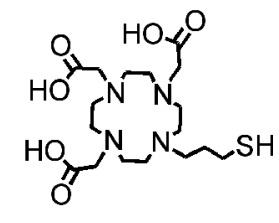

8

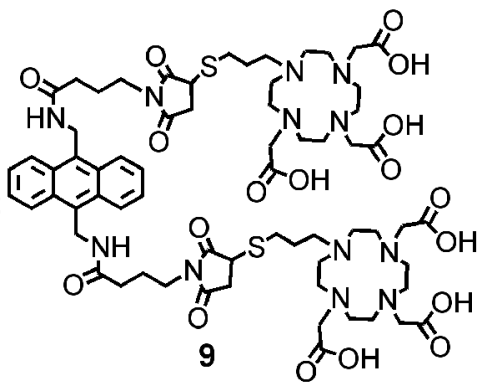

Scheme 6.3. Reaction scheme showing the formation of the bis-macrocyclic ligand ANT2DO3A (9) by means of a thiol-maleimide Michael-type addition.

The paramagnetic and fluorescent properties were integrated to the ANT2DO3A (9) ligand by simultaneous complexation with $\mathrm{Gd}^{3+}$ and $\mathrm{Eu}^{3+}$, respectively. This reaction was carried out in the presence of $50 \% \mathrm{GdCl}_{3}$ and $50 \% \mathrm{EuCl}_{3}$ (Scheme 6.4) in water ( $\mathrm{pH} \mathrm{6}$, adjusted with $\left.1 \mathrm{M} \mathrm{NaOH}\right)$ at $50^{\circ} \mathrm{C}$ for 24 hours. Owing to the assumed equal lanthanide affinity by the ANT-2DO3A ligand, the formation of three complexes, i.e., the paramagnetic $2 \mathrm{Gd}^{3+}$-ANT-2DO3A, the fluorescent $2 \mathrm{Eu}^{3+}-\mathrm{ANT}-2 \mathrm{DO} 3 \mathrm{~A}$ and the multimodal $\mathrm{Gd}^{3+} / \mathrm{Eu}^{3+}-\mathrm{ANT}-2 \mathrm{DO} 3 \mathrm{~A}$, is expected. 


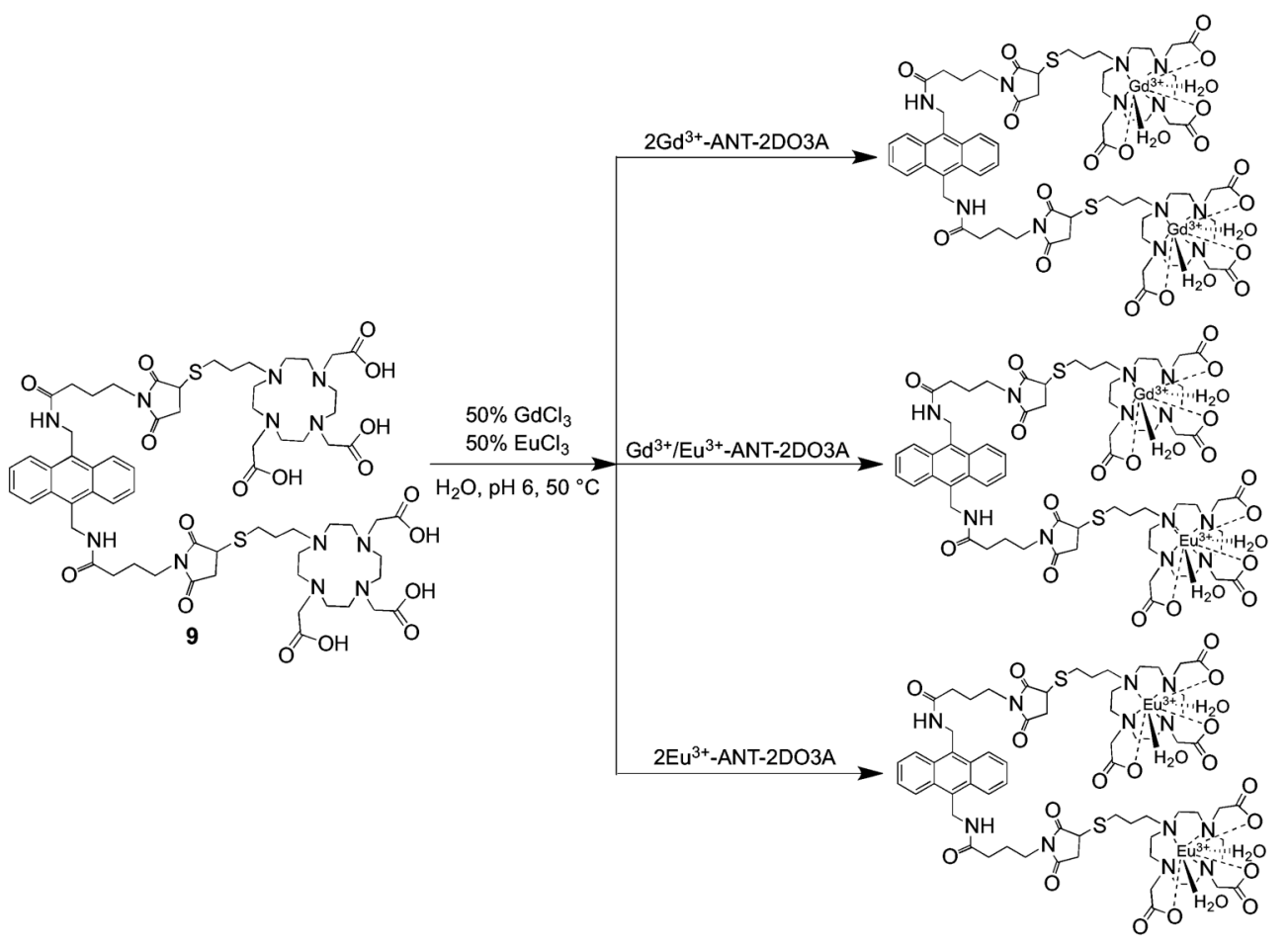

Scheme 6.4. Reaction scheme showing the three possible complexes formed after complexation of 9 with $50 \% \mathrm{GdCl}_{3}$ and $50 \% \mathrm{EuCl}_{3}$.

6.2.4 Characterization of the Final Bis-Macrocyclic ANT-2DO3A Ligand and its Lanthanide Complexes

All intermediates of the two building blocks shown in Scheme 6.1 and Scheme 6.2 were characterized using ${ }^{1} \mathrm{H}$ and ${ }^{13} \mathrm{C}$ nuclear magnetic resonance (NMR) and electrospray (ESI)/matrix-assisted laser desorption ionization (MALDI) mass spectrometry (MS) (data can be found in the experimental section and Appendix 6.1).

Interestingly, the molecular weight of the water-soluble ANT-2DO3A ligand $(1406.6 \mathrm{~g} / \mathrm{mol}$ ) facilitates its purification by dialysis. When using a $1000 \mathrm{Da}$ dialysis membrane, low percentages of 1) the mono-macrocyclic ligand (anthracene-core building block $\mathbf{3}$ with only one DO3A-based ligand 8 172 
attached to it) and 2) the DO3A-based ligand 8 dimer (resulting from possible disulfide bridge formation) are likely to be present in the final solution as both species have molecular weights below (but close) the molecular cut-off of the membrane (988.4 and $838.4 \mathrm{~g} / \mathrm{mol}$ respectively.).

Full characterization of the final bis-macrocyclic ligand ANT-2DO3A (9, Scheme 6.3) proved to be challenging due to various constraints: MS data remain inconclusive (as no $\mathrm{m} / \mathrm{z}$ ratio peak could be assigned to the final molecule or to any fragment) and the water solubility of ANT-2DO3A was lower than that required for ${ }^{13} \mathrm{C}$ NMR spectroscopy. Still, characterization of ANT-2DO3A was possible using ${ }^{1} \mathrm{H}$ NMR spectroscopy. However, the restricted rotational motion of the full molecule, together with the rigidity of the DO3A cyclen core made it difficult to identify and assign all proton signals properly. As shown in Figure 6.1 (top), the ${ }^{1} \mathrm{H}$ NMR spectrum shows signals on both the aromatic and the aliphatic region. Whereas the aromatic peaks correspond to the anthracene core, those in the aliphatic region agree with typical DO3A ${ }^{1} \mathrm{H}$ NMR spectra, where broad signals are often observed due to hindered rotation. ${ }^{35}$ This observation is supported by experiments at higher temperatures $\left(50^{\circ} \mathrm{C}\right.$, Figure 6.1 bottom), where only the resolution of the peaks corresponding to the DO3A-based ligand increases (highlighted area). No changes in the ${ }^{1} \mathrm{H}$ NMR spectra were observed after 8 months, thereby confirming the stability of ANT-2DO3A in solution. 


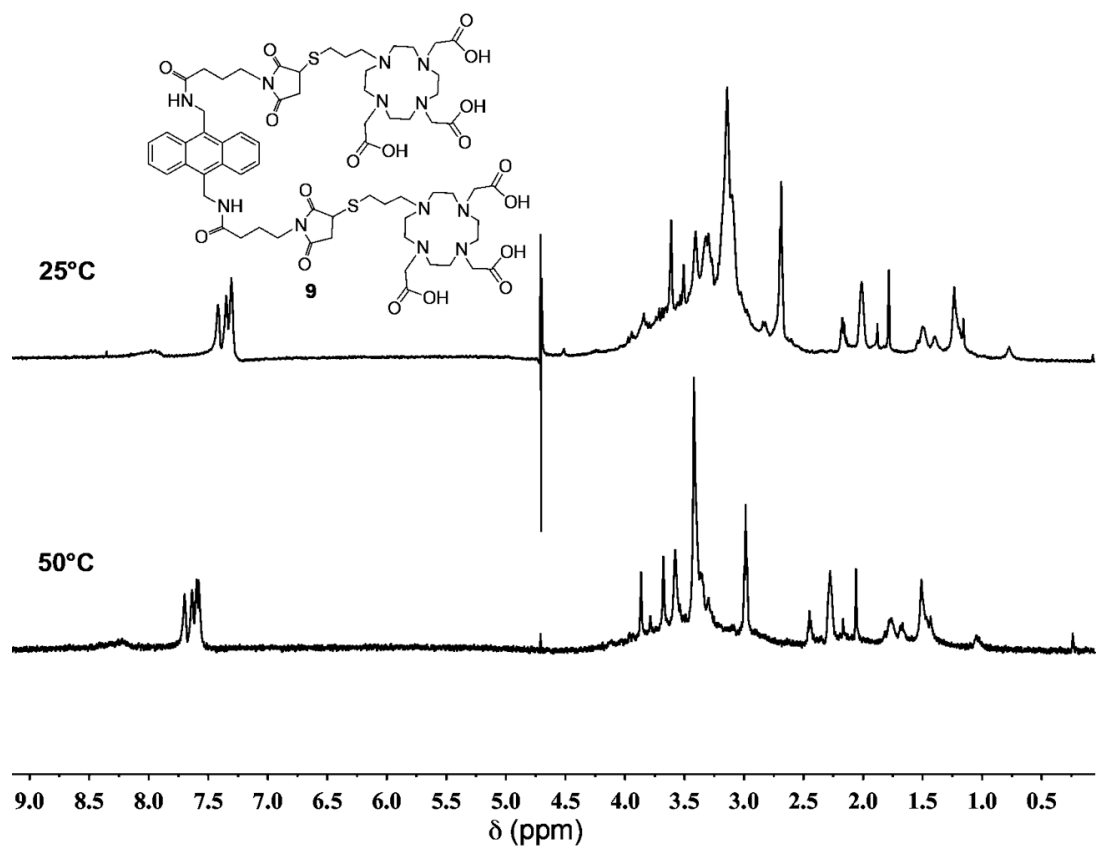

Figure 6.1. ${ }^{1} \mathrm{H}$ NMR spectrum of ANT-2DO3A (9) in $\mathrm{D}_{2} \mathrm{O}$ at 25 and $50^{\circ} \mathrm{C}$.

Further studies using 2D NMR techniques (COSY AND HMQC) led to the assignment of several of the proton signals observed in Figure 6.1. As shown in Figure 6.2, the aromatic (a, b), amide (d), and DO3A protons (i, h, $\mathbf{m}, \mathbf{n}, \mathbf{0}, \mathbf{p}, \mathbf{q}, \mathbf{r})$ were identified at $7.42-7.31,8$ and $4-2.75$ ppm, respectively. Also, protons $\mathbf{e}, \mathbf{f}$ and $\mathbf{g}$ were assigned to the propyl alkylated maleimide linker and the signal corresponding to the $\mathbf{c}$ protons was found to coincide with the water signal (intentionally suppressed in this spectrum). While little is known about the spatial location of the DO3A building block (8), upon reaction with $\mathbf{3}$, the signals corresponding to the thiolated propyl linker (j, $\mathbf{k}$ and I) protons are expected to show splitting (non-equivalency) due to the presence of two chiral centers (highlighted with black circles in Figure 6.2). The proton signals observed at 2.18, 1.5, $1.38,1.23$ and $0.78 \mathrm{ppm}$ are thought to correspond to the thiolated propyl 
linker (j, k and I). Interestingly, the splitting observed for these signals show that the symmetry in the molecule is lost only after the chiral centers. Furthermore, integration of the assigned signals revealed that the value obtained for the aromatic signals (8 protons, 7.42-7.31 ppm) matches those obtained for the propyl alkylated maleimide linker (2.70 and 2.02 ppm, 4 protons each, 3.10 cannot be integrated) and the thiolated propyl linker (total of 12 protons).

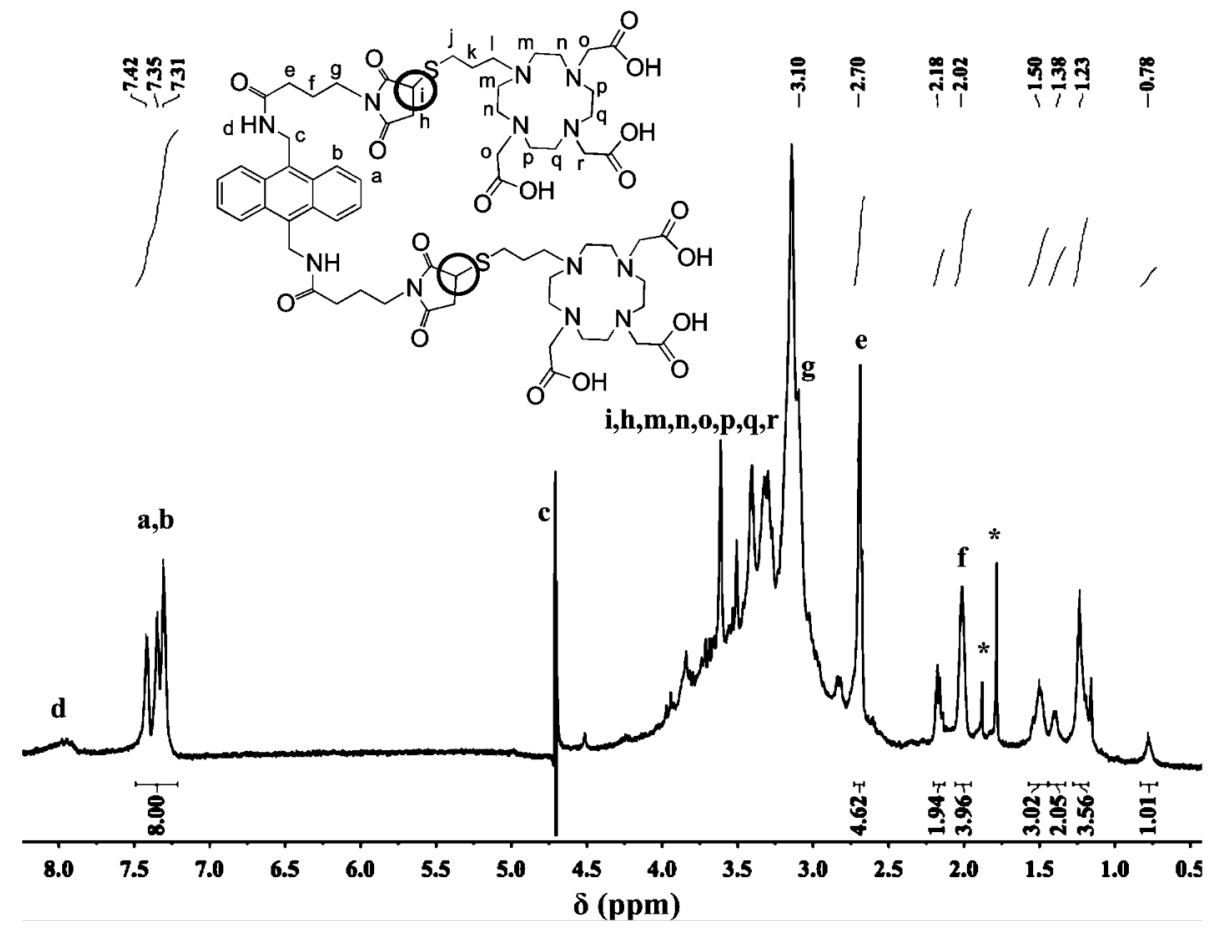

Figure 6.2. ${ }^{1} \mathrm{H}$ NMR spectrum and peak assignment of ANT-2DO3A at $25^{\circ} \mathrm{C}$. Signals from dialysis membrane residual are indicated with an asterisk $\left(^{*}\right)$ and chiral centers are indicated with black circles.

Matrix-assisted laser desorption ionization (MALDI) MS characterization of the $\mathrm{Gd}^{3+} / \mathrm{Eu}^{3+}$-ANT-2DO3A complex suggests the formation of a $\mathrm{Gd}^{3+}$ containing complex. As shown in Figure 6.3, the peak with a $\mathrm{m} / \mathrm{z}$ value of 
976.52 was successfully identified as a fragment of the final compound, namely one resulting from cleavage of one of the amide bonds connecting the propyl alkylated maleimide to the anthracene core (theoretical molecular weight: 976.28). Furthermore, this peak shows a Gaussian-like isotope pattern consistent with lanthanide-containing species (due to the various abundant isotopes) (Figure 6.3 , inset), most likely $\mathrm{Gd}^{3+}$ as it has more isotopes than $\mathrm{Eu}^{3+}$ (7 vs. 2).

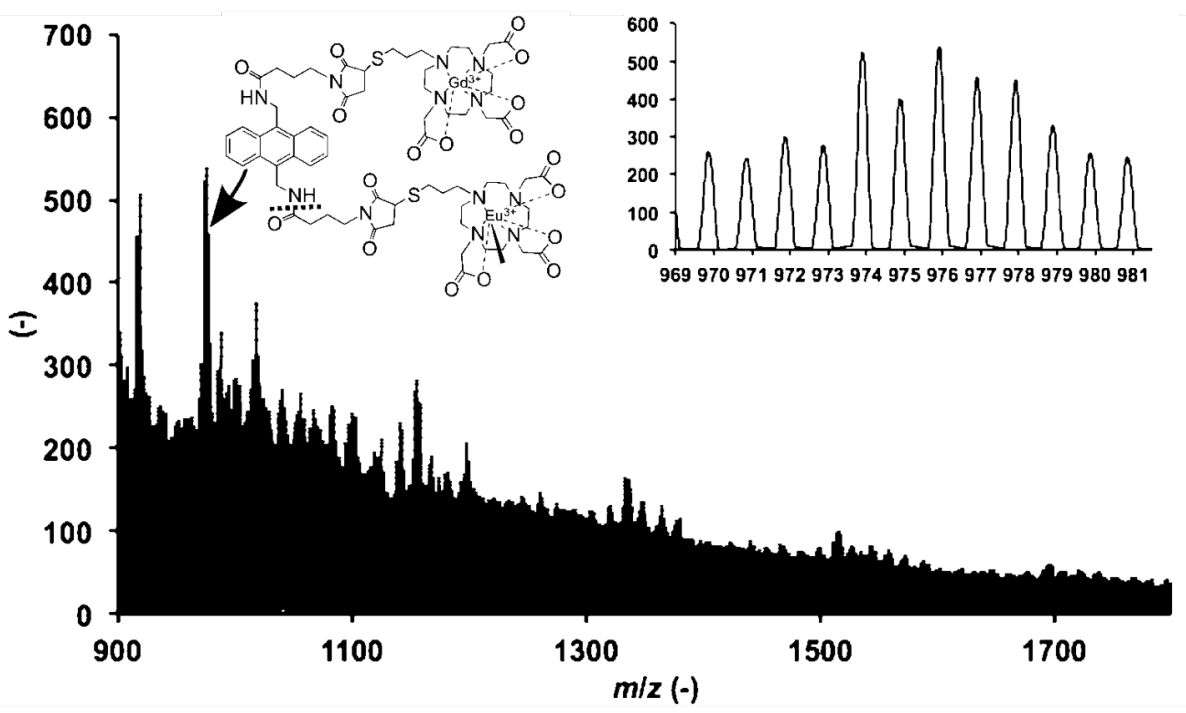

Figure 6.3. MALDI-MS spectrum of $\mathrm{Gd}^{3+} / \mathrm{Eu}^{3+}-\mathrm{ANT}-2 \mathrm{DO} 3 \mathrm{~A}$. The peak with the highest intensity was observed at $\mathrm{m} / \mathrm{z} 976.52$. Inset: amplification of the region between $\mathrm{m} / \mathrm{z} 969$ and 982.

Despite the various challenges faced in order to fully characterize the ANT2DO3A ligand and its $\mathrm{Gd}^{3+} / \mathrm{Eu}^{3+}$ complex, and encouraged by the promising MS results, the paramagnetic and optical properties of the suspected $\mathrm{Gd}^{3+} / \mathrm{Eu}^{3+}-\mathrm{ANT}-2 \mathrm{DO} 3 \mathrm{~A}$ complex were studied. 


\subsubsection{Optical Properties}

The optical properties of the ANT-2DO3A ligand and its $\mathrm{Gd}^{3+} / \mathrm{Eu}^{3+}$ complex were investigated using UV/Vis absorbance and steady-state fluorescence spectroscopy. As shown in Figure 6.4 (black line), a broad absorption band with maximum at $312 \mathrm{~nm}$ was observed for the ANT-2DO3A ligand in $\mathrm{H}_{2} \mathrm{O}$. Note that in $\mathrm{H}_{2} \mathrm{O}$, the fine structure (vibronic peaks corresponding to different vibrational sub-levels) usually seen for anthracene in non-polar solvents is not observed. Upon lanthanide complexation, the absorption spectrum shows a hypsochromic (blue) shift with a maximum at $270 \mathrm{~nm}$ (green line), thereby suggesting a change in the absorption properties of the ANT-2DO3A ligand. In particular, the $\pi$-electron system of the anthracene core is strongly affected by the incorporation of the lanthanide. $^{36}$ For comparative purposes, the absorption spectrum of $\mathrm{Gd}^{3+} / \mathrm{Eu}^{3+}$-ANT-2DO3A in $\mathrm{H}_{2} \mathrm{O} / \mathrm{ACN}$ is also shown (red line). In this case, the anthracene-like fine structure is observed, with maxima at 269 and 262 $\mathrm{nm}$.

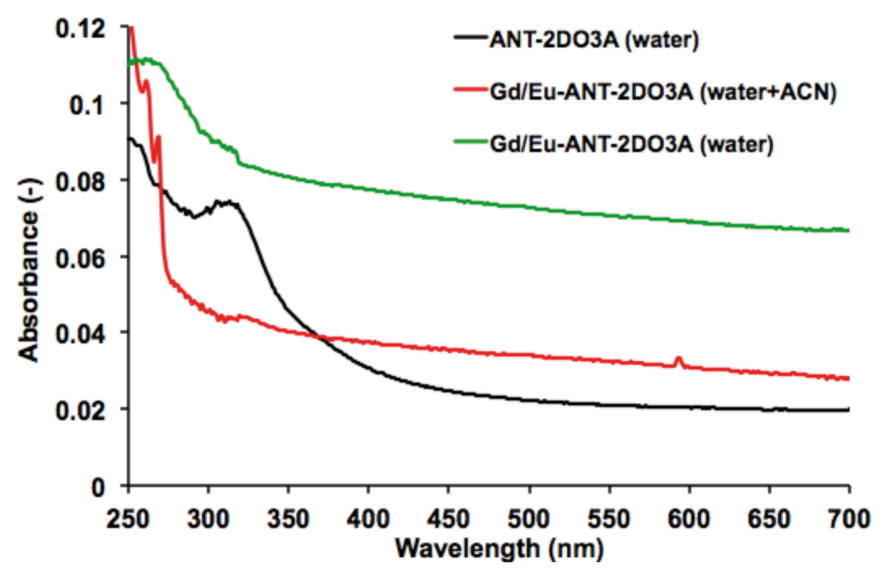

Figure 6.4. UV absorption spectra of the ligand ANT-2DO3A in $\mathrm{H}_{2} \mathrm{O}$ (black line) and the complex $\mathrm{Gd}^{3+} / \mathrm{Eu}^{3+}$-ANT-2DO3A in $\mathrm{H}_{2} \mathrm{O}$ (green line) and $\mathrm{H}_{2} \mathrm{O} / \mathrm{ACN}$ (red line). 
In case of $\mathrm{Eu}^{3+}$ complexation by the ANT-2DO3A ligand, energy transfer from the anthracene core to the chelated lanthanide is expected. However, how efficient this process will be is unknown since little is understood about the final conformation of the complex (how close the chelates are to the antenna) and if the length of the linkage between building blocks 3 and 8 affects it. Therefore, possible fluorescence emission resulting from energy-transfer from the anthracene to the $\mathrm{Eu}^{3+}$ complex was studied by steady-state fluorescence spectroscopy. In Figure 6.5, the emission spectrum of the ANT-2DO3A ligand in $\mathrm{H}_{2} \mathrm{O}$ (grey line) is presented, showing anthracene emission (when exciting at $\lambda=312 \mathrm{~nm}$ ) with a maximum at $442 \mathrm{~nm}$. Moreover, anthracene-like emission was also observed in the $\mathrm{Gd}^{3+} / \mathrm{Eu}^{3+}$-ANT-2DO3A complex sample $\left(\mathrm{H}_{2} \mathrm{O} / \mathrm{ACN}\right.$, black line), when exciting at $262 \mathrm{~nm}$, with maxima at 397, 419 and $561 \mathrm{~nm}$. A broad peak, with maxima at $600 \mathrm{~nm}$ and two shoulders at 590 and 615 $\mathrm{nm}$, was also observed. Although the peaks could not be resolved, this region is consistent with bands observed for sensitized $\mathrm{Eu}^{3+}$ complexes and associated to ${ }^{5} \mathrm{D}_{0} \rightarrow 7 \mathrm{~F}_{1}(591 \mathrm{~nm}),{ }^{5} \mathrm{D}_{0} \rightarrow 7 \mathrm{~F}_{2}(615 \mathrm{~nm})$ and ${ }^{5} \mathrm{D}_{0} \rightarrow 7 \mathrm{~F}_{3}$ $(658 \mathrm{~nm})$ transitions. ${ }^{26,36,37}$ Thus, these results strongly suggest the formation of an $\mathrm{Eu}^{3+}$ complex with a conformation of the final ANT-2DO3A complex such that, despite the distance between the anthracene moiety and the $\mathrm{Eu}^{3+}$ complex, energy transfer occurs. 


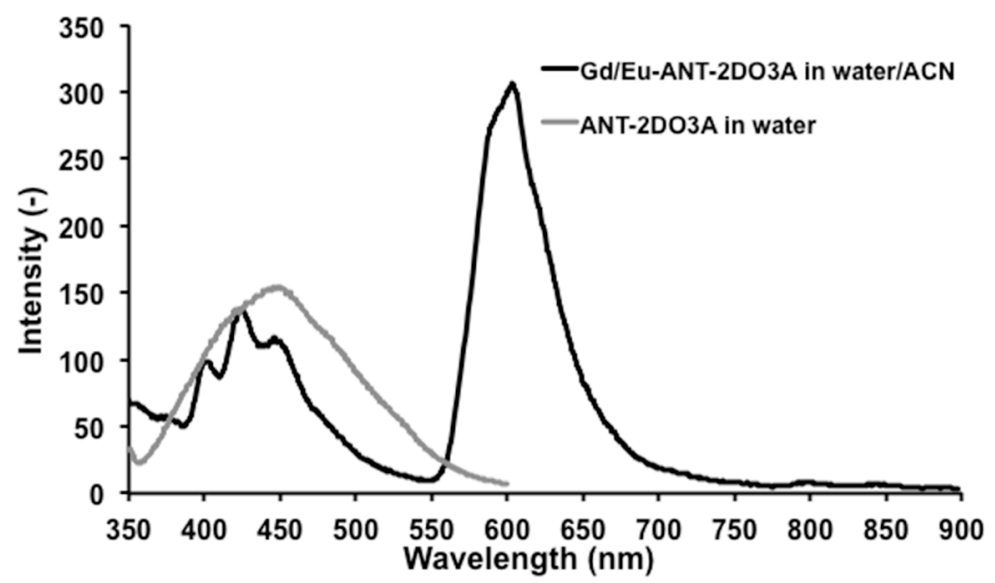

Figure 6.5. Emission spectra of ANT-2DO3A (grey line, $\mathrm{H}_{2} \mathrm{O}, \lambda_{\mathrm{exc}}=312 \mathrm{~nm}$ ) and $\mathrm{Gd}^{3+} / \mathrm{Eu}^{3+}$ ANT-2DO3A (black line, $\mathrm{H}_{2} \mathrm{O} / \mathrm{ACN}, \lambda_{\text {exc }}=262 \mathrm{~nm}$ ).

\subsubsection{Paramagnetic Properties}

Shortened longitudinal relaxation $\left(T_{1}\right)$, resulting from the formation of a $\mathrm{Gd}^{3+}$ complex, was studied using MRI. As shown in Figure 6.6, enhanced $T_{1}$ contrast for a series of dilutions of $\mathrm{Gd}^{3+} / \mathrm{Eu}^{3+}-\mathrm{ANT}-2 \mathrm{DO} 3 \mathrm{~A}$ was observed, thereby confirming the paramagnetic properties of the complex. While the two lowest concentrations $\left(0.4\right.$ and $0.8 \mathrm{~g} / \mathrm{L} \mathrm{Gd}^{3+} / \mathrm{Eu}^{3+}-\mathrm{ANT}-$ 2DO3A) appear to be within the detection limit, the highest two clearly show contrast differences when compared to the control $\left(\mathrm{H}_{2} \mathrm{O}\right)$. 


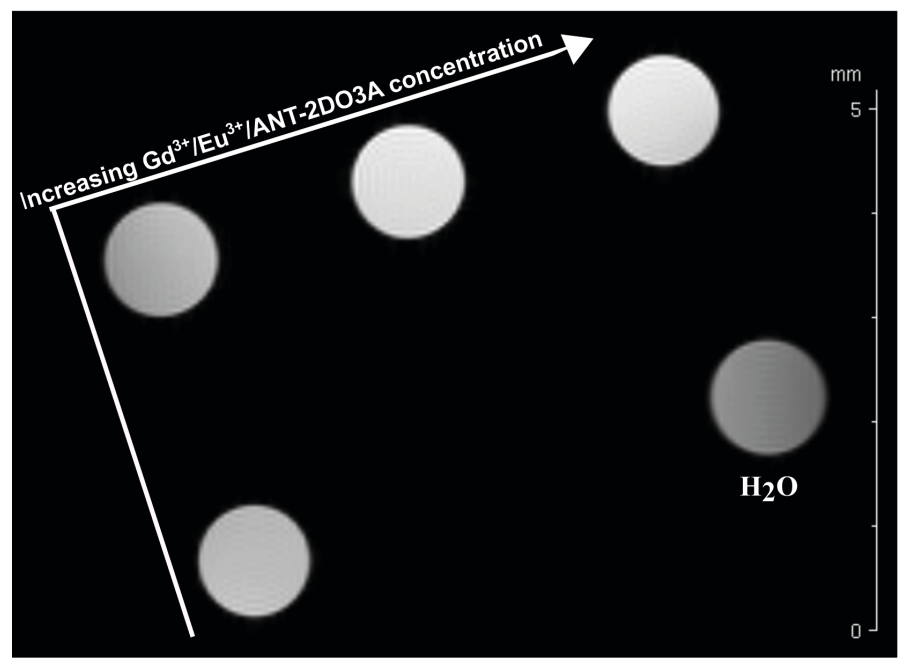

Figure 6.6. $T_{1}$-weighted MR Image showing various concentrations $(4,2,0.8$ and $0.4 \mathrm{~g} / \mathrm{L})$ of $\mathrm{Gd}^{3+} / \mathrm{Eu}^{3+}$-ANT-2DO3A complex and $\mathrm{H}_{2} \mathrm{O}$ (control).

\subsection{Carbon Nanotubes (CNTs) for Multimodal Molecular Imaging: a Preliminary Study}

To demonstrate the versatility of the 1,4,7,10-tetraaza-1-(1-aminobutyl)4,7,10-triacetic acid cycloddodecane (AB-DO3A, Chapter 4) ligand, the nanoparticle growth-mediator template $A B-D O 3 A$ was attached to carbon nanotubes (CNTs) and used for metal (lanthanide) complexation.

\subsubsection{Design and Synthesis}

Given the wide variety of current approaches for covalent functionalization of CNTs, ${ }^{38}$ the incorporation of AB-DO3A to SWCNTs was explored. Under the hypothesis that the free amine of the $4^{\text {th }}$ pendant arm of $A B-D O 3 A$ can be incorporated to any nanostructure, CNTs are an advantageous archetype nanomaterial. ${ }^{39-41}$ In the past ten years, the increased water solubility (and stability) of oxidized SWCNTs (ox-SWXCNTs) ${ }^{42}$ has opened 
up the doors for various biological applications, including multimodal imaging. ${ }^{39,41,43,44}$ Indeed, while known for their intrinsic $T_{2}$ relaxation effect, ${ }^{44}$ one of the first examples of CNT-based $T_{1}$ relaxation agents were the gadonanotubes described by Hartman et al..$^{43}$ These single-walled carbon nanotubes (SWCNTs) contained small paramagnetic clusters of $\mathrm{Gd}^{3+}$ ions residing in the sidewall defects of the nanotubes. In another example, Richard et al. ${ }^{44}$ reported non-covalently functionalized multi-walled carbon nanotubes (MWCNTs) using amphiphilic $\mathrm{Gd}^{3+}$-DTPA-based chelates. Encouraged by their high surface-to-volume ratio, making them excellent platforms for the attachment of large loads of (various) molecular imaging probes, SWCNTs were functionalized with AB-DO3A, whose water solubility is enhanced by the three carboxylic acids of this ligand. Subsequently, AB-DO3A-SWCNTs were used as chelates for lanthanide complexation. Interestingly, since lanthanides are characterized by their differences in properties (paramagnetic $\mathrm{Gd}^{3+}$ vs. fluorescent $\mathrm{Eu}^{3+}$ ), ${ }^{13-17} \mathrm{AB}-$ DO3A-SWCNTs are excellent platforms for magnetic resonance/optical multimodal imaging. The final SWCNTs were characterized by means of ${ }^{1} \mathrm{H}$ NMR and infrared (IR) spectroscopy, vibrating sample magnetometry (VSM), transmission electron microscopy (TEM) and energy-dispersive $X$ ray (EDX) spectroscopy.

\subsubsection{Covalent Functionalization of CNTs}

The reaction scheme followed for the synthesis of $\mathrm{Ln}^{3+}-\mathrm{AB}-\mathrm{DO} 3 \mathrm{~A}$ SWCNTS is shown in Scheme 6.5. Here, the free amine of the AB-DO3A ligand serves as an excellent handle for amide formation with ox-SWCNTs using standard protocols (in water). ${ }^{39}$ The AB-DO3A-SWCNTs obtained were dialyzed and allowed to bind to $\mathrm{Gd}^{3+}$ (for $\mathrm{MRI}$ ) and $\mathrm{Eu}^{3+}$ (for optical imaging) in order to form the desired complexes. The water solubility of all 
the intermediates and the final product allows the use of simple dialysis protocols for purification of the final nanomaterial.

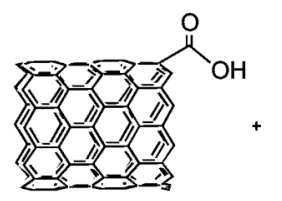

OX-SWCNT

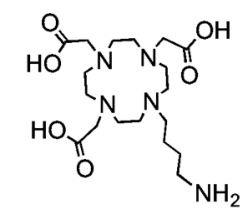

$\mathrm{AB}-\mathrm{DO} 3 \mathrm{~A}$

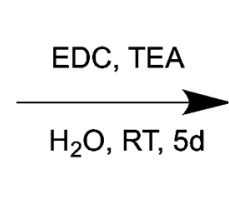

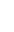

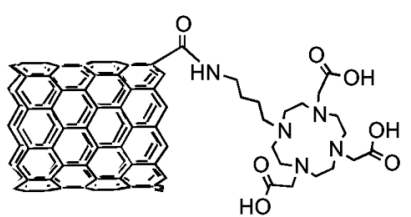

AB-DO3A-SWCNT

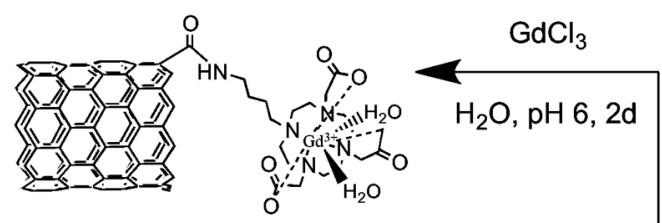

Gd+3-AB-DO3A-SWCNT

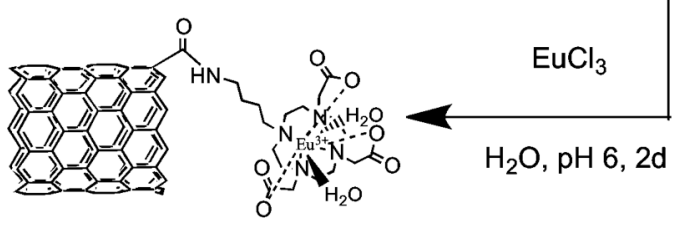

$\mathrm{Eu}^{3+}-\mathrm{AB}-\mathrm{DO} 3 \mathrm{~A}-\mathrm{SWCNT}$

Scheme 6.5. Synthesis of $\mathrm{Ln}^{3+}-\mathrm{AB}-\mathrm{DO} 3 \mathrm{~A}-\mathrm{SWCNTS}$.

The AB-DO3A functionalized SWCNTs were characterized using ${ }^{1} \mathrm{H}$ NMR and IR spectroscopy. The ${ }^{1} \mathrm{H}$ NMR spectrum corresponding to the functionalized AB-DO3A-SWCNTs (Figure 6.7, top) shows the presence of various resonance peaks in the aliphatic region. These peaks are shifted and broadened when compared those observed in the ${ }^{1} \mathrm{H}$ NMR spectrum of AB-DO3A (Figure 6.7, bottom). This last feature can be attributed to a slower molecular rotation due to attachment to bigger scaffolds (in this case the ox-SWCNTs), making it difficult to fully assign all protons. 
Additionally, the peaks corresponding to the closest protons to the free amine (3 ppm) are not observed after functionalization (highlighted area). These two observations strongly suggest the successful functionalization of ox-SWCNTs with the AB-DO3A ligand.

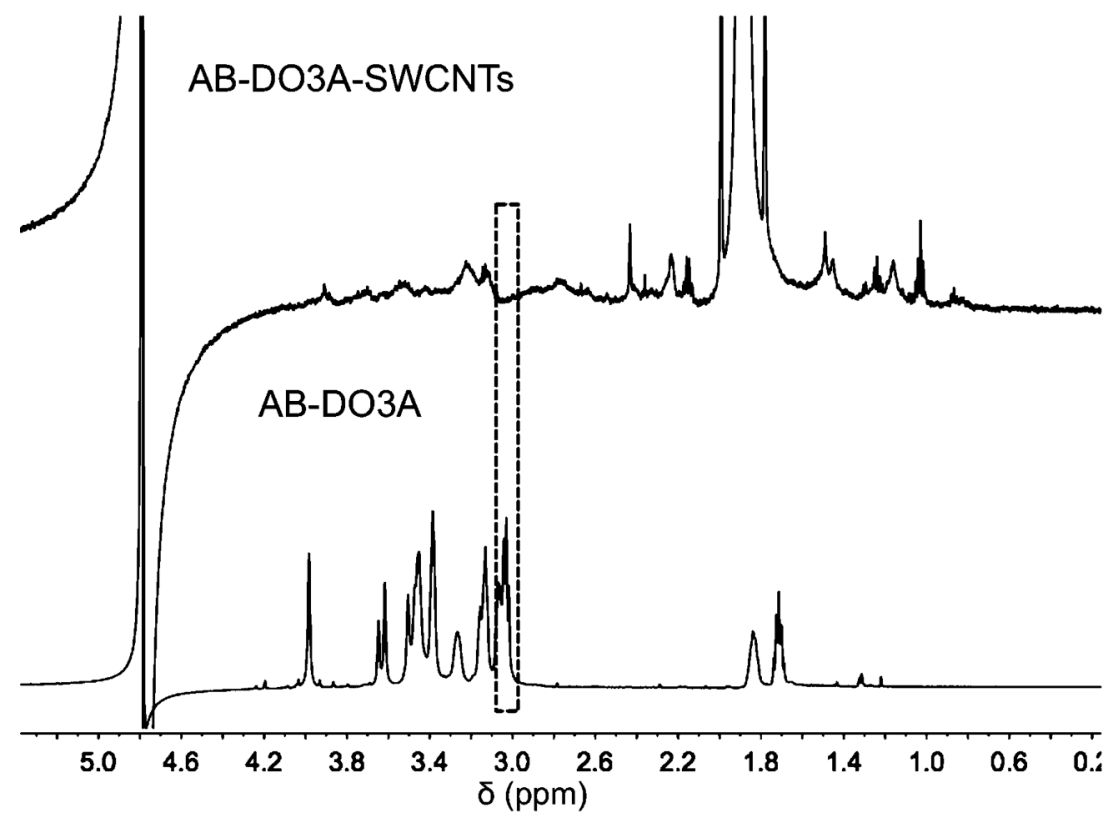

Figure 6.7. ${ }^{1} \mathrm{H}$ NMR spectra of functionalized AB-DO3A-SWCNTs (top) and AB-DO3A (bottom) in $\mathrm{D}_{2} \mathrm{O}$ at $25^{\circ} \mathrm{C}$.

IR spectroscopic studies further supported the apparent surface functionalization of ox-SWCNTs. As shown in Figure 6.8, ox-SWCNTs (grey line) show vibrational bands at $1715 \mathrm{~cm}^{-1}(\mathrm{C}=\mathrm{O}$ stretch, carboxylic acids on the ox-SWCNTs surface) and $1565 \mathrm{~cm}^{-1}$ (C=C bend, SWCNTs). After functionalization, the vibrational band at $1715 \mathrm{~cm}^{-1}$ (black line) corresponds to remaining (non-functionalized) carboxylic acids. However, the appearance of a more intense, broad vibrational band at $1534 \mathrm{~cm}^{-1}$ with a shoulder at $1633 \mathrm{~cm}^{-1}$ strongly suggests changes in the structure of 
the SWCNTs ${ }^{45}$ due to amide formation (1690-1630 $\mathrm{cm}^{-1}$ ) resulting from the functionalization with $\mathrm{AB}-\mathrm{DO} 3 \mathrm{~A}$.

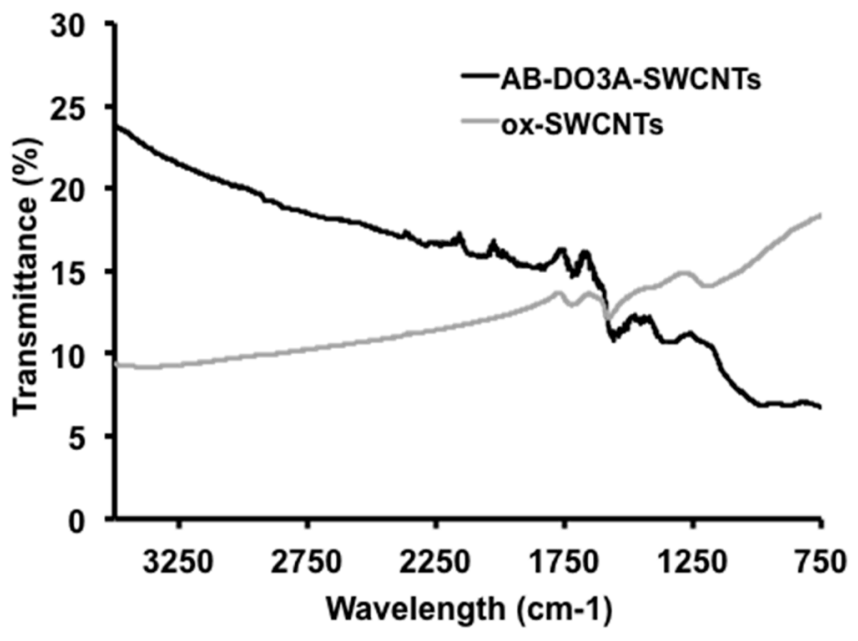

Figure 6.8. IR- spectra of ox-SWCNTs (grey line) and AB-DO3A-SWCNTs (black line).

\subsubsection{Lanthanide Complexation using AB-DO3A-SWCNTS}

The AB-DO3A-SWCNTs were subsequently allowed to react with $\mathrm{Gd}^{3+}$ and $\mathrm{Eu}^{3+}$ salts in order to provide paramagnetic and optical properties, respectively, to them. Changes in the magnetic properties of the $\mathrm{Gd}^{3+}-\mathrm{AB}$ DO3A-SWCNTs were measured using a vibrating sample magnetometer (VSM). Pristine SWCNTs are super paramagnetic, most likely due to the presence of transition metals ( $\mathrm{Fe}$ and $\mathrm{Ni}$ ) used in catalysts for their synthesis (spectrum not shown). A significant decrease in magnetization is observed upon oxidation of the SWCNTs (Figure 6.9, left), showing that the catalyst debris is removed during the oxidation step. The very low saturation magnetization of the ox-SWCNTs facilitates the observation of the diamagnetic component (due to the carbon of the SWCNTs): a negative slope (at saturation) is observed. The positive slope of the 
magnetization (at saturation) observed, as well as the disappearance of the diamagnetic component, is indicative of paramagnetic $\mathrm{Gd}^{3+}-\mathrm{AB}-\mathrm{DO} 3 \mathrm{~A}-$ SWCNTs (Figure 6.9, right). The manifestation of a paramagnetic component strongly suggests the presence of $\mathrm{Gd}^{3+}$ complexes on the surface of the ox-SWCNTs.
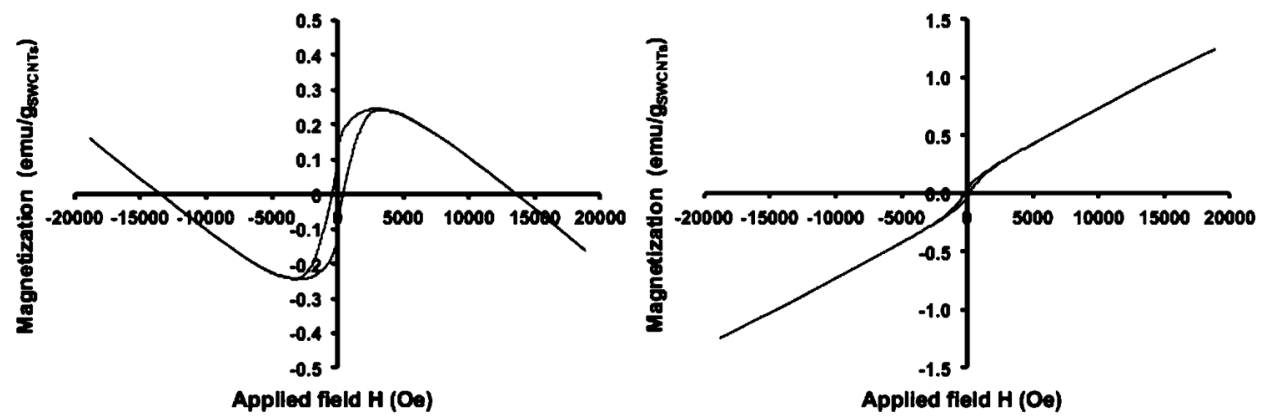

Figure 6.9. Magnetization curves of Ox-SWCNTs (left), and $\mathrm{Gd}^{3+}-\mathrm{AB}-\mathrm{DO} 3 \mathrm{~A}-\mathrm{SWCNTS}$ (right).

Finally, the resulting $\mathrm{Ln}^{3+}-\mathrm{AB}-\mathrm{DO} 3 \mathrm{~A}-\mathrm{SWCNTS}$ were visualized by TEM (Figure 6.10), and the presence of $\mathrm{Gd}^{3+}$ and $\mathrm{Eu}^{3+}$ on the surface of the SWCNTs was studied using EDX spectroscopy. The insets of Figure 6.10 show the EDX spectra of $\mathrm{Gd}^{3+}-\mathrm{AB}-\mathrm{DO} 3 \mathrm{~A}-\mathrm{SWCNTS}$ (left) and $\mathrm{Eu}^{3+}-\mathrm{AB}$ DO3A-SWCNTs (right). In both cases, X-ray emission at the appropriate lanthanide energy was observed, thereby confirming the presence of these species on the surface of the functionalized SWCNTs. In each TEM image, $\mathrm{X}$-ray emission at the energy corresponding to $\mathrm{Eu}^{3+}$ or $\mathrm{Gd}^{3+}$ was not observed for selected background regions. 

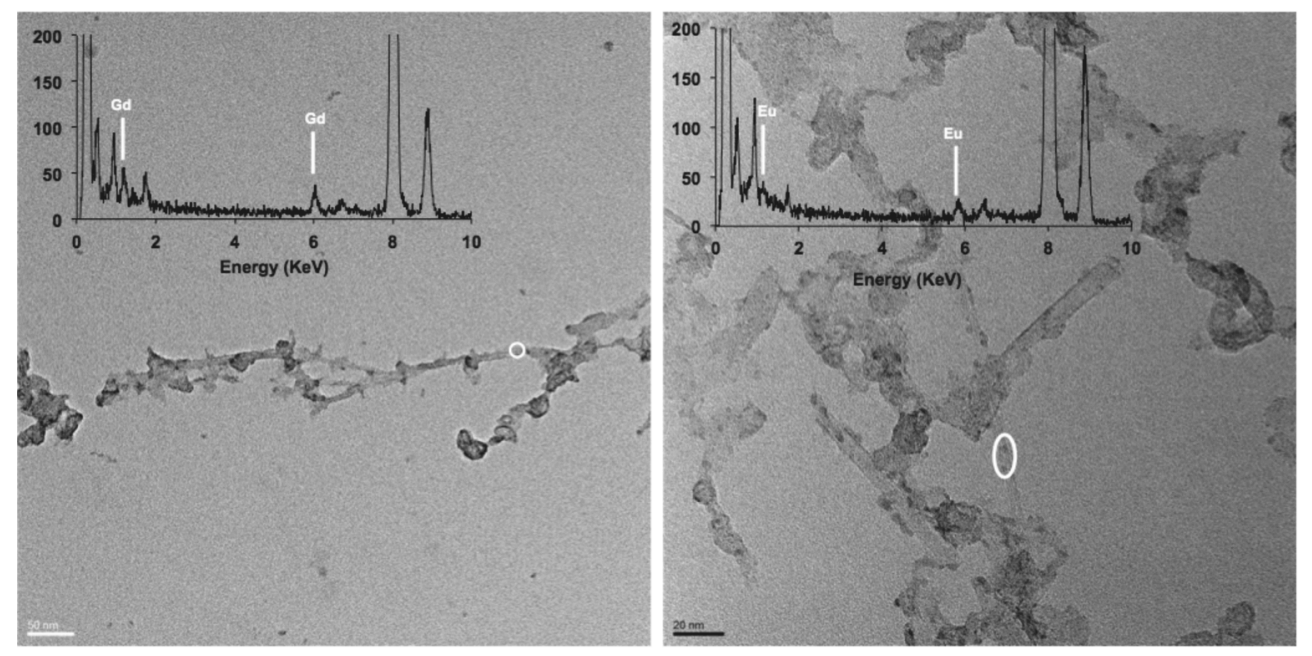

Figure 6.10. TEM-images of $\mathrm{Gd}^{3+}-\mathrm{AB}-\mathrm{DO} 3 \mathrm{~A}-\mathrm{SWCNTS}$ (left) and $\mathrm{Eu}^{3+}-\mathrm{AB}-\mathrm{DO} 3 \mathrm{~A}-\mathrm{SWCNTS}$ (right) (scale bar left $=50 \mathrm{~nm}$ and right $=20 \mathrm{~nm}$ ). Inset in both pictures is the EDX spectrum of the regions highlighted in white.

While this example highlights the versatility of the metal complex-based templates developed in previous chapters, there are several concerns and challenges to be overcome. Furthermore, the incorporation of extra features such as a targeting/therapeutical moiety or simply other combinations of imaging modalities is, intuitively, the next step to follow.

\subsection{Challenges in Metal Complex-Based Templates for Magnetic Resonance/Optical Multimodal Imaging Agents}

\subsubsection{Characterization}

Proper characterization of these imaging probes remains a difficult task. Typically, a combination of routine techniques for small organic molecules 
(e.g., NMR, IR, MS, HPLC) and state-of-the-art techniques for nanomaterials (e.g., TEM, SEM, XPS, etc.) is used. However, the former techniques not always provide full information (as shown in Chapter 3 ) and the latter ones strongly depend on, e.g., sample preparation (AuNRs in Chapter 5). Furthermore, several of these probes suffer from solubility issues, making their characterization even more problematic. The challenge remains finding the right set of techniques that offer sufficient accurate and valuable information.

\subsubsection{Other Imaging Techniques}

The ligands presented in this thesis show great flexibility as they can be used to complex other metal ions and isotopes, resulting in new properties for other modalities such as nuclear imaging. Moreover, the metal nanoparticles described here are known for their use as IAs for X-ray computed tomography (CT), various optical imaging modalities and photo acoustic imaging. ${ }^{46-52}$ This versatility makes several of the IAs shown here suitable for alternative multimodal imaging modalities (e.g., PET-MRI). ${ }^{51,53-59}$

\subsubsection{Targeting}

The molecular imaging probes described in previous chapters are nonspecific and, therefore, suffer from a lack of specificity as intracellular IAs. A straightforward approach to overcome this issue is by incorporating celltargeting moieties to these probes in order to "direct them" towards cells. ${ }^{60}$ Throughout the years, cellular targeting has been achieved using peptides, ${ }^{1,6,61-64}$ antibodies ${ }^{7,61,62}$ and proteins ${ }^{62,65-69}$ that bind to receptors at cell membrane surfaces, e.g., somatostatin/transferrin receptors. ${ }^{70}$ Several examples of such systems are illustrated in Chapter 2. 


\subsection{What's (Really) Next?}

Upon identification of potential IAs (those that deliver proper contrast for visualization and exhibit cell-specificity by highlighting targeted areas), the next intuitive step is to provide some degree of therapeutical properties to them. In this way, IAs are not only used for visualization of ROIs, but can also serve as carriers for drug delivery or for therapeutical purposes (e.g, ultrasound). This field, known as theranostics, plays a major role in earlystage drug design and development and is expected to contribute to personalized medicine. ${ }^{71,72}$

\subsection{Conclusions}

The preliminary results presented here show the potential of the functionalization of carbon-based scaffolds for multimodal imaging purposes. Although the aim was to synthesize a small molecule multifunctional imaging ligand for complexation with 2 different lanthanides, the full characterization of the final molecule by means of $1 \mathrm{D}$ and 2D NMR turned out to be inconclusive. However, MS data pointed to the formation of the aimed ligand. While the exact stoichiometry of the final complex could not be elucidated, the studied properties of this new multimodal IA strongly suggest the formation of both complexes (either in one molecule and/or separated). Therefore, future efforts should focus on the full characterization of such ligands/complexes, as they can be the first step towards a lanthanide-controlled synthesis of heteronuclear multimodal IAs. On the other hand, $\mathrm{Gd}^{3+} / \mathrm{Eu}^{3+}-\mathrm{AB}-\mathrm{DO} 3 \mathrm{~A}-\mathrm{SWCNT}$ are expected to be excellent candidates for high-payload multimodal imaging purposes. Intuitively, the next step is the assessment of the fluorescent 
properties of these SWCNTs and their visualization by MRI and fluorescence imaging.

Lastly, the combination of simple modifications of widely known polydentate ligands for lanthanide complexation with nanomaterials has opened up the door for new synthetic routes for (multimodal) IAs. The current knowledge of metal complexation, together with commercial availability of many nanomaterials, has made this modest approach popular. Present efforts are directed towards a more comprehensive characterization of these systems, their cell targeting ability and their use for other state-of-the-art imaging modalities.

\subsection{Fxperimental}

Materials. Commercial grade reagents were purchased from Sigma Aldrich and TCl Europe and were used without further purification unless otherwise stated. The CNTs used were pristine high purity SWCNTs from Arrynano (purity $>90 \%$ according to supplier). The outer diameter of the SWCNTs ranges between $1 \mathrm{~nm}$ and $2 \mathrm{~nm}$ and their length from $5 \mu \mathrm{m}$ to 20 $\mu \mathrm{m}$. Solvents were dried over molecular sieves. Deuterated solvents were purchased from Cambridge Isotope Laboratories and NMR and MRI tubes from Wilmad LabGlass. All CNTs were oxidized and functionalized using 18.2 $\mathrm{M} \Omega / \mathrm{cm}^{2}$ water. The $1000 \mathrm{Da}$ and 12000-14000 Da Spectra/Por ${ }^{\circledR}$ dialysis membranes were purchased from Aldrich.

9,10-Bis (aminomethyl)anthracene (1). This compound was synthesized following an adapted procedure from reference. ${ }^{33}$ Hexamethylenetetramine $(1720 \mathrm{mg}, 12.3 \mathrm{mmol})$ was added to a solution of 9,10-bis(bromomethyl)anthracene (synthesized as described in reference) ${ }^{34}$ 
(2 g, $5.6 \mathrm{mmol})$ in $100 \mathrm{~mL} \mathrm{CHCl}$. After refluxing overnight, the precipitate was removed by filtration and washed with $\mathrm{CHCl}_{3}$. The resulting solid was added to $400 \mathrm{~mL}$ of a $8: 1 \mathrm{CH}_{3} \mathrm{CH}_{2} \mathrm{OH}: \mathrm{HCl}$ solution, which was subsequently refluxed for two days. The solution was cooled to $0^{\circ} \mathrm{C}$, filtered and the remaining solid was washed with cold $\mathrm{CH}_{3} \mathrm{CH}_{2} \mathrm{OH}(3 \mathrm{x}$ $80 \mathrm{~mL}$ ) and dried under vacuum. The product was dispersed in $200 \mathrm{~mL}$ water $(\mathrm{pH}>11$, adjusted using $1 \mathrm{M} \mathrm{NaOH})$ and extracted with $\mathrm{CHCl}_{3}(3 \mathrm{x}$ $120 \mathrm{~mL}$ ). Finally, the organic phase was dried over $\mathrm{MgSO}_{4}$, the solvent was removed under reduced pressure and the remaining yellow solid was dried overnight in vacuum over $\mathrm{P}_{2} \mathrm{O}_{5}$, yielding 1. Yield: $86 \%$ (1.14 g, $\left.4.8 \mathrm{mmol}\right)$. ${ }^{1} \mathrm{H}$ NMR (600 MHz, $\mathrm{CDCl}_{3}, 25^{\circ} \mathrm{C}$, TMS) $\delta(\mathrm{ppm}): 8.4\left[\mathrm{dd}, J_{1}(\mathrm{H}, \mathrm{H})=3.7 \mathrm{~Hz}\right.$, $J_{2}(\mathrm{H}, \mathrm{H})=3.3 \mathrm{~Hz}, 4 \mathrm{H}$; Ar- $H$ ], $7.6\left[\mathrm{dd}, J_{1}(\mathrm{H}, \mathrm{H})=3.7 \mathrm{~Hz}, J_{2}(\mathrm{H}, \mathrm{H})=3.3 \mathrm{~Hz}, 4 \mathrm{H}\right.$; Ar-H], 4.9 (s, 4H, $\mathrm{NH}_{2}-\mathrm{CH}_{2}-\mathrm{Ar}$ ). ${ }^{13} \mathrm{C} \mathrm{NMR}\left(150 \mathrm{MHz}, \mathrm{CDCl}_{3}, 25^{\circ} \mathrm{C}, \mathrm{TMS}\right)$ $\delta$ (ppm): 134.7, 129.3, 125.7, $124.6(\mathrm{Ar}-\mathrm{C}), 38.4\left(\mathrm{NH}_{2}-\mathrm{CH}_{2}-\mathrm{Ar}\right)$.

\section{9,10-Bis (methyl-4-bromobutanamide) anthracene}

(2).

4 equivalents of $N, N$ '-diisopropylethylamine (DIPEA) were added to $200 \mathrm{~mL}$ dry $\mathrm{CHCl}_{3}$ containing 9,10-bis(aminomethyl)anthracene

(500 mg, $2.1 \mathrm{mmol}$ ) under $\mathrm{N}_{2}$ atmosphere. Subsequently, 4-bromobutyryl chloride (1180 mg, $6.4 \mathrm{mmol}$ ) was slowly added to the solution, which was stirred overnight in the dark. The precipitate was filtered, washed with $\mathrm{CHCl}_{3}$ and dried under vacuum, yielding 2 as a yellow solid. Yield: $75 \%$ (852 mg, $1.6 \mathrm{mmol}) .{ }^{1} \mathrm{H}$ NMR (600 MHz, DMSO- $d_{6}, 25^{\circ} \mathrm{C}$, TMS) $\delta$ (ppm): 8.4 $\left[\mathrm{dd}, J_{1}(\mathrm{H}, \mathrm{H})=3.4 \mathrm{~Hz}, J_{2}(\mathrm{H}, \mathrm{H})=3.4 \mathrm{~Hz}, 4 \mathrm{H} ; \mathrm{Ar}-\mathrm{H}\right]$ \& $(\mathrm{t}, J(\mathrm{H}, \mathrm{H})=5.2 \mathrm{~Hz}, 2 \mathrm{H}$, $\mathrm{CONH}-\mathrm{CH}_{2}-\mathrm{Ar}$ ), 7.6 [dd, $J_{1}(\mathrm{H}, \mathrm{H})=3 \mathrm{~Hz}, J_{2}(\mathrm{H}, \mathrm{H})=3.7 \mathrm{~Hz}, 4 \mathrm{H}$; Ar-H], 5.3 (s, $4 \mathrm{H}, \mathrm{CONH}-\mathrm{CH}_{2}-\mathrm{Ar}$ ), 3.5 (t, $\mathrm{J}(\mathrm{H}, \mathrm{H})=6.6 \mathrm{~Hz}, 4 \mathrm{H} ; \mathrm{NHCO}-\mathrm{CH}_{2}-\mathrm{CH}_{2}-\mathrm{CH}_{2} \mathrm{Br}$ ), $2.2\left(\mathrm{t}, \mathrm{J}(\mathrm{H}, \mathrm{H})=7.1 \mathrm{~Hz}, 4 \mathrm{H}\right.$; NHCO- $\left.\mathrm{CH}_{2}-\mathrm{CH}_{2}-\mathrm{CH}_{2} \mathrm{Br}\right), 2\left(\mathrm{~m}, \mathrm{~J}_{1}(\mathrm{H}, \mathrm{H})=7 \mathrm{~Hz}\right.$, $\left.J_{2}(\mathrm{H}, \mathrm{H})=7 \mathrm{~Hz}, \quad 4 \mathrm{H} ; \quad \mathrm{NHCO}-\mathrm{CH}_{2}-\mathrm{CH}_{2}-\mathrm{CH}_{2} \mathrm{Br}\right) .{ }^{13} \mathrm{C} \quad \mathrm{NMR}(150 \mathrm{MHz}$, 
DMSO- $\left.d_{6}, 25^{\circ} \mathrm{C}, \mathrm{TMS}\right) \delta$ (ppm): 171.1 (CONH-Ar), 130.4, 127.5, 126.5, 125.3 ( $\mathrm{Ar}-\mathrm{C}), 35.6$ (CONH-CH$-\mathrm{Ar}), 35\left(\mathrm{NHCO}-\mathrm{CH}_{2}-\mathrm{CH}_{2}-\mathrm{CH}_{2} \mathrm{Br}\right), 33.9$ $\left(\mathrm{NHCO}-\mathrm{CH}_{2}-\mathrm{CH}_{2}-\mathrm{CH}_{2} \mathrm{Br}\right), \quad 29.1 \quad\left(\mathrm{NHCO}-\mathrm{CH}_{2}-\mathrm{CH}_{2}-\mathrm{CH}_{2} \mathrm{Br}\right) . \quad \mathrm{MS} \quad(\mathrm{ESI}+$, $\left.\mathrm{CH}_{2} \mathrm{Cl}_{2} / \mathrm{MeOH}\right): \mathrm{m} / \mathrm{z}: 455.5,457.5\left[\mathrm{M}^{+}-1 \mathrm{Br}+\mathrm{H}\right], 555.5,557.5,559.5\left[\mathrm{M}^{+}+\mathrm{Na}\right]$.

\section{9,10-Bis [methyl-4-N-(butanamide) maleimide] anthracene}

(3). Maleimide $(36.3 \mathrm{mg}, 0.37 \mathrm{mmol})$ and $\mathrm{K}_{2} \mathrm{CO}_{3}(44 \mathrm{mg}, 0.32 \mathrm{mmol})$ were added to a $10 \mathrm{~mL}$ dry ACN solution containing 9,10-bis(methyl-4bromobutanamide)anthracene (2) (100 $\mathrm{mg}, 0.19 \mathrm{mmol})$. The mixture was heated at $50^{\circ} \mathrm{C}$ overnight and left to cool to room temperature. After filtration in order to remove inorganic salts, the solvent was removed under reduced pressure. Recrystallization from $\mathrm{MeOH}$ yielded $\mathbf{3}$ as a white solid. Yield: $80 \%$ (95.4 mg, $0.17 \mathrm{mmol}) .{ }^{1} \mathrm{H}$ NMR (600 MHz, $\mathrm{CDCl}_{3}, 25^{\circ} \mathrm{C}, \mathrm{TMS}$ ) $\delta$ (ppm): 7.6.-7.1 [dd, 12H; $\mathrm{Ar}-\mathrm{H}$ \& $\mathrm{CH}=\mathrm{CH}$ maleimide], 4.4 (t, $J(\mathrm{H}, \mathrm{H})=7 \mathrm{~Hz}, 4 \mathrm{H} ; \mathrm{NHCO}-\mathrm{CH}_{2}-\mathrm{CH}_{2}-\mathrm{CH}_{2} \mathrm{Br}$ ), 2.7 (m, 4H; NHCO- $\mathrm{CH}_{2}-\mathrm{CH}_{2}-$ $\mathrm{CH}_{2} \mathrm{Br}$ ), 2.2 (m, 4H; NHCO- $\mathrm{CH}_{2}-\mathrm{CH}_{2}-\mathrm{CH}_{2} \mathrm{Br}$ ). ${ }^{13} \mathrm{C} \mathrm{NMR}\left(150 \mathrm{MHz}, \mathrm{CDCl}_{3}\right.$, $\left.25^{\circ} \mathrm{C}, \mathrm{TMS}\right) \delta$ (ppm): 176.2 (CONH-Ar), 163.9 ( $\mathrm{C}=\mathrm{O}$ maleimide), 143.9, 141.2, 126.3, 125.6, 123.6 (Ar- $\mathrm{C} \& \mathrm{CH}=\mathrm{CH}$ maleimide), $70.4\left(\mathrm{NHCO}^{-\mathrm{CH}_{2}-}\right.$ $\left.\mathrm{CH}_{2}-\mathrm{CH}_{2} \mathrm{Br}\right), 45.3$ (CONH-CH$\left.-\mathrm{Ar}\right), 28.9\left(\mathrm{NHCO}-\mathrm{CH}_{2}-\mathrm{CH}_{2}-\mathrm{CH}_{2} \mathrm{Br}\right), 23.6$ $\left(\mathrm{NHCO}-\mathrm{CH}_{2}-\mathrm{CH}_{2}-\mathrm{CH}_{2} \mathrm{Br}\right) . \quad \mathrm{MS} \quad\left(\mathrm{ESI}+, \quad \mathrm{CH}_{2} \mathrm{Cl}_{2} / \mathrm{MeOH}\right): \quad \mathrm{m} / \mathrm{z}: 235.6$ [bisaminoanthracene+H] (frag), $470.2\left[\mathrm{M}^{+}-1\right.$ maleimide+H] (frag), 567.2 $\left[\mathrm{M}^{+}+\mathrm{H}\right]$. Elemental analysis, found: C 68.7, H 5.96, N 8.22, O 17.12. Expected for $\mathrm{C}_{32} \mathrm{H}_{30} \mathrm{~N}_{4} \mathrm{O}_{6} \bullet 0.4 \mathrm{CH}_{3} \mathrm{CH}_{2} \mathrm{OH}$ : C 67.34, $\mathrm{H}$ 5.58, N 9.58, O 17.5.

2-((3-Chloropropyl) thio)tetrahydro-2H-pyran (5). This compound was synthesized following an adapted procedure from literature. ${ }^{73} 3$ chloro-1-propanethiol (4) (500 mg, $4.5 \mathrm{mmol}$ ), 3,4-dihydro-2H-pyran $(410 \mu \mathrm{L}, 4.5 \mathrm{mmol})$ and pyridinium $p$-toluene sulfonate $(113 \mathrm{mg}, 0.45$ 
mmol) were dissolved in $30 \mathrm{~mL}$ dry $\mathrm{CH}_{2} \mathrm{Cl}_{2}$ and the reaction mixture was stirred at room temperature overnight. The solution was washed with $10 \%$ $\mathrm{NaOH}(3 \times 20 \mathrm{~mL})$, water $(3 \times 20 \mathrm{~mL})$, and dried over $\mathrm{MgSO}_{4}$. The solvent was removed under reduced pressure and the desired compound was obtained as a yellow liquid after chromatography on silica gel 60 (pretreated with $0.1 \%$ TEA in hexane) using toluene as eluent ( $\mathrm{rf} \sim 0.4)$. Yield: $47 \%$ (414 mg, $2.1 \mathrm{mmol}) .{ }^{1} \mathrm{H}$ NMR (600 MHz, $\left.\mathrm{CDCl}_{3}, 25^{\circ} \mathrm{C}, \mathrm{TMS}\right) \delta$ (ppm): $4.9(\mathrm{~m}, 1 \mathrm{H} ; \mathrm{SCHO}), 4.1 \& 3.5\left(\mathrm{~m}, 2 \mathrm{H} ; \mathrm{OCH}_{2}\right), 3.7[\mathrm{t}, \mathrm{J}(\mathrm{H}, \mathrm{H})=6.2 \mathrm{~Hz}, 2 \mathrm{H}$; $\mathrm{CH}_{2} \mathrm{Cl}$, $2.9 \& 2.8\left(\mathrm{~m}, 2 \mathrm{H} ; \mathrm{CH}_{2} \mathrm{~S}\right), 2.1\left(\mathrm{~m}, \mathrm{~J}(\mathrm{H}, \mathrm{H})=6.8,6.7 \mathrm{~Hz}, 2 \mathrm{H} ; \mathrm{CH}_{2^{-}}\right.$ $\left.\mathrm{CH}_{2} \mathrm{Cl}\right), 2 \& 1.7\left(\mathrm{SCH}-\mathrm{CH}_{2}\right), 1.8 \& 1.6\left(\mathrm{~m}, 2 \mathrm{H} ; \mathrm{SCH}-\mathrm{CH}_{2} \mathrm{CH}_{2}\right), 1.6(\mathrm{~m}, 2 \mathrm{H}$; $\left.\left.\mathrm{OCH}_{2}-\mathrm{CH}_{2}\right) .{ }^{13} \mathrm{C} \mathrm{NMR} \mathrm{(150} \mathrm{MHz,} \mathrm{CDCl}_{3}, 25^{\circ} \mathrm{C}, \mathrm{TMS}\right) \delta$ (ppm): 82.1 (SCHO), $64.4\left(\mathrm{OCH}_{2}\right), 43.4\left(\mathrm{CH}_{2} \mathrm{Cl}\right), 32.2\left(\mathrm{CH}_{2} \mathrm{Cl}-\mathrm{CH}_{2}\right), 30.6\left(\mathrm{SCH}-\mathrm{CH}_{2}\right), 27.1\left(\mathrm{CH}_{2} \mathrm{~S}\right)$, $25.4\left(\mathrm{OCH}_{2}-\mathrm{CH}_{2}\right), 21.5\left(\mathrm{SCH}-\mathrm{CH}_{2} \mathrm{CH}_{2}\right)$. MS (ESI+, $\left.\mathrm{CH}_{2} \mathrm{Cl}_{2} / \mathrm{MeOH}\right): \mathrm{m} / \mathrm{z}$ : 195.2, $197.2\left[\mathrm{M}^{+}+\mathrm{H}\right], 217.2,219.2\left[\mathrm{M}^{+}+\mathrm{Na}\right]$.

\section{1-(3-( (Tetrahydro-2H-pyran-2yl)thio) propyl)-4,7, 10-tris (tert-} butoxy carbonylmethyl)-1,4,7,10-tetraazacyclododecane (7). In a three-neck round-bottom flask, $\mathrm{K}_{2} \mathrm{CO}_{3}(621 \mathrm{mg}, 4.5 \mathrm{mmol})$ and a catalytic amount of $\mathrm{KI}$ were added to a stirring solution of 1,4,7-tris(tert-butoxy carbonylmethyl)-1,4,7,10-tetraazacyclododecane 6 (DO3A, synthesized as previously described in literature $\left.{ }^{18}\right)(790 \mathrm{mg}, 1.5 \mathrm{mmol})$ in $80 \mathrm{~mL}$ dry acetonitrile. The mixture was kept under $\mathrm{N}_{2}$ for 10 minutes while vigorously stirring. Subsequently, 3-chloro-1-propyltetrahydropyranyl sulfide (5) (300 mg, $1.5 \mathrm{mmol}$ ) dissolved in $20 \mathrm{~mL}$ dry $\mathrm{CH}_{2} \mathrm{Cl}_{2}$ was added slowly to the reaction mixture and allowed to react for 3 days at $50^{\circ} \mathrm{C}$. The mixture was left to cool to room temperature, filtered to remove inorganic salts and the solvent was evaporated under reduced pressure. The desired compound was obtained as a yellow oil after chromatography on silica gel 60 (pre- 
treated with $0.1 \%$ TEA in hexane) using $\mathrm{CH}_{2} \mathrm{Cl}_{2} / \mathrm{MeOH}$ 9:1 as eluent $\left(\mathrm{R}_{\mathrm{f}} \sim\right.$ $0.5)$, followed by precipitation of impurities with ether, filtration and solvent evaporation. Yield: $50 \%$ (538 mg, $0.8 \mathrm{mmol})$. ${ }^{1} \mathrm{H} \mathrm{NMR}\left(600 \mathrm{MHz}, \mathrm{CDCl}_{3}\right.$, $\left.25^{\circ} \mathrm{C}, \mathrm{TMS}\right) \delta(\mathrm{ppm}): 4.8(\mathrm{~m}, 1 \mathrm{H} ; \mathrm{SCHO}), 4 \& 3.6\left(\mathrm{~m}, 2 \mathrm{H} ; \mathrm{OCH}_{2}\right), 3.5-2.7[\mathrm{~m}$, $24 \mathrm{H} ; \mathrm{NCH}_{2}$ (cyclen core), $\mathrm{NCH}_{2}$ (4th arm), $\mathrm{CH}_{2}-\mathrm{COOtBu}, 2.73(\mathrm{~m}, 2 \mathrm{H}$; $\left.\mathrm{CH}_{2} \mathrm{~S}\right), 2.1\left(\mathrm{~m}, 2 \mathrm{H}, \mathrm{CH}_{2} \mathrm{~S}-\mathrm{CH}_{2}\right), 1.9 \& 1.61\left(\mathrm{~m}, 2 \mathrm{H} ; \mathrm{SCH}-\mathrm{CH}_{2}\right), 1.82 \& 1.56$ (m, 2H; $\left.\left.\mathrm{SCH}-\mathrm{CH}_{2} \mathrm{CH}_{2}\right), 1.56\left(\mathrm{~m}, 2 \mathrm{H} ; \mathrm{OCH}_{2}-\mathrm{CH}_{2}\right), 1.46\left[\mathrm{~s}, 27 \mathrm{H} ; \mathrm{C}\left(\mathrm{CH}_{3}\right)_{3}\right)\right]$. ${ }^{13} \mathrm{C}$ NMR (150 MHz, $\left.\mathrm{CDCl}_{3}, 25^{\circ} \mathrm{C}, \mathrm{TMS}\right) \delta$ (ppm): 170.7, 170.3, 170, (COOtBu), $83.4(\mathrm{SCHO}), 82\left[\mathrm{C}\left(\mathrm{CH}_{3}\right)_{3}\right], 66.2\left(\mathrm{OCH}_{2}\right), 56.9,55.3,54.7,53.2$, $52.7,51.8,51,50.2,47.9$ [ $\mathrm{NCH}_{2}$ (all), $\left.\mathrm{NCH}_{2}-\mathrm{CH}_{2}, \mathrm{CH}_{2} \mathrm{COOtBu}\right], 31.7$ (SCH$\left.\mathrm{CH}_{2}\right), 28.3\left(\mathrm{CH}_{2} \mathrm{~S}-\mathrm{CH}_{2}\right), 28.1\left[\mathrm{C}\left(\mathrm{CH}_{3}\right)_{3}\right], 25.1\left(\mathrm{CH}_{2} \mathrm{~S}\right), 23.5\left(\mathrm{SCH}-\mathrm{CH}_{2} \mathrm{CH}_{2}\right)$, $22.5\left(\mathrm{OCH}_{2}-\mathrm{CH}_{2}\right)$. MS (ESI+, $\left.\mathrm{CH}_{2} \mathrm{Cl}_{2} / \mathrm{MeOH}\right): \mathrm{m} / \mathrm{z}: 695.2\left[\mathrm{M}^{+}+\mathrm{Na}\right]$.

\section{1-(3-Mercaptopropyl)-4,7,10-triacetic}

acid-1,4,7,10tetraazacyclododecane (8). In a round-bottom flask, TFA $(5 \mathrm{~mL})$ was added to a solution of $7(200 \mathrm{mg}, 0.3 \mathrm{mmol})$ in $5 \mathrm{~mL} \mathrm{CH}_{2} \mathrm{Cl}_{2}$ and the reaction mixture was allowed to stir at room temperature overnight. The mixture was evaporated under reduced pressure and the residue coevaporated with $\mathrm{CH}_{2} \mathrm{Cl}_{2} 3$ times in order to remove excess TFA. 8 was obtained as a solid after precipitation from diethyl ether and it was subsequently used for the next reaction. Yield: quantitative. MS (ESI+, 1:1 ACN:0.1\%TFA): $m / z: 421.2\left[\mathrm{M}^{+}+\mathrm{H}\right]$.

\section{9,10-Bis [methyl-1-C(4- (N)-butanamide-2,5-dioxopyrrolidin-3- yl)thio) propyl)-4,7,10-triacetic acid-1,4,7,10-} tetraazacyclododecane] anthracene (9). $3(50 \mathrm{mg}, 0.09 \mathrm{mmol})$ and 8 (74 mg, $0.18 \mathrm{mmol}$ ) were dissolved in $20 \mathrm{~mL} \mathrm{MeOH}$. Subsequently, the solution was flushed with $\mathrm{N}_{2}$ and allowed to stir overnight. After solvent 
removal under reduced pressure, the remaining powder was redissolved in water and dialyzed (against $\mathrm{H}_{2} \mathrm{O}$ ) for three days using a 1.000 Da dialysis membrane. After water removal under reduced pressure and drying (overnight) under vacuum over $\mathrm{P}_{2} \mathrm{O}_{5}, 9$ was obtained as a yellow solid. Yield: $5 \%$ (6 mg, $0.0043 \mathrm{mmol}) .{ }^{1} \mathrm{H}$ NMR (600 MHz, $\mathrm{D}_{2} \mathrm{O}, 25^{\circ} \mathrm{C}, \mathrm{TMS}$ ) $\delta$ (ppm): 8 (amide), 7.42-7.31 (m, 8H, Ar-H), $4.6\left(\mathrm{CONH}_{-} \mathrm{CH}_{2}-\mathrm{Ar}\right), 4-2.75$, [m, $\mathrm{NCH}_{2}$ (cyclen core), $\mathrm{CH}_{2}-\mathrm{COOH}$ and $\mathrm{CH}_{2}-\mathrm{CH}$ maleimide], $3.10\left(\mathrm{~m}, \mathrm{CH}_{2}-\right.$ $\mathrm{CH}_{2}-\mathrm{CH}_{2}$-maleimide), 2.7 (t, $\mathrm{J}(\mathrm{H}, \mathrm{H})=6.7 \mathrm{~Hz}, 4 \mathrm{H}, \mathrm{CH}_{2}-\mathrm{CH}_{2}-\mathrm{CH}_{2}$-maleimide), $2.02\left(\mathrm{~m}, 4 \mathrm{H}, \mathrm{CH}_{2}-\mathrm{CH}_{2}-\mathrm{CH}_{2}\right.$-maleimide), 2.18, 1.5, 1.38, 1.23, $0.78(\mathrm{~m}, 12 \mathrm{H}$, $\mathrm{CH}_{2}-\mathrm{CH}_{2}-\mathrm{CH}_{2}$-S-). The final molecule (as well as possible fragments) could not be identified by MS. Due to the little amount of material elemental analysis was not possible.

\section{$\mathrm{Gd}^{3+} / \mathrm{Fu}^{3+}-\{$ 9,10-Bis [methyl-1-C(4- (N-butanamide-2,5-}

dioxopyrrolidin-3-yl)thio) propyl)-4,7,10-triacetic acid-1,4,7,10tetraazacyclododecane] anthracene (10). $\mathrm{EuCl}_{3}$ and $\mathrm{GdCl}_{3}(2.4 \mathrm{mg}$, $0.0064 \mathrm{mmol})$ were added to a solution of 9 (6 mg, $0.0043 \mathrm{mmol})$ in $15 \mathrm{~mL}$ $\mathrm{H}_{2} \mathrm{O}$. The $\mathrm{pH}$ of the solution was adjusted to 6 using $1 \mathrm{M} \mathrm{NaOH}$ and the final solution was stirred at $50^{\circ} \mathrm{C}$ for one day. Excess salts were removed by dyalizis against $\mathrm{H}_{2} \mathrm{O}$ for three days using a 1.000 Da dialysis membrane. After solvent removal under reduced pressure, $\mathbf{1 0}$ was obtained as a pale yellow solid. Yield: quantitative. MS+ (MALDI, $\mathrm{H}_{2} \mathrm{O} / \mathrm{ACN}+0.1 \%$ TFA, $\beta$-indoleacrylic acid $\left.{ }^{74}\right): m / z: 976.52\left[\mathrm{M}^{+}-(\mathrm{C}=\mathrm{O}\right.$-propylalkylated maleimidethiolated propyl linker-DO3Acomplex)+H] (frag).

Synthesis of AB-DO3A. As described in Chapter 4. 
Oxidation procedure for SWCNTs. Full procedure is described in the PhD thesis of Martin Sobik (UTwente).

\begin{abstract}
AB-D03A-SWCNTs. ${ }^{39}$ AB-DO3A $(50 \mathrm{mg}, 0.12 \mathrm{mmol}), \quad 1-(3-$ dimethylaminopropyl)-3-ethylcarbodiimide $\mathrm{HCl} \quad(\mathrm{EDC} \times \mathrm{HCl}, 76.5 \mathrm{mg}$, $0.4 \mathrm{mmol}$ ) and diisopropylethylamine (DIPEA, $160 \mu \mathrm{l}, 1 \mathrm{mmol}$ ) were added to a suspension of $10 \mathrm{mg}$ of ox-SWNT in $25 \mathrm{ml}$ of water. The reaction mixture was allowed to stir at room temperature overnight and dialyzed against $\mathrm{H}_{2} \mathrm{O}$ for 3 days using a 12000-14000 Da membrane in order to remove the excess of unreacted reactants.
\end{abstract}

$\mathbf{G d}^{3+} / \mathbf{E u}^{3+}$-AB-DO3A-SWCNTs. $\mathrm{EuCl}_{3}$ or $\mathrm{GdCl}_{3}(8 \mathrm{mg}, 0.04 \mathrm{mmol})$ was added to a solution of AB-SWCNTs (5 mg each solution) in $10 \mathrm{~mL} \mathrm{H}_{2} \mathrm{O}$. The $\mathrm{pH}$ of the solution was adjusted to 6 using $1 \mathrm{M} \mathrm{NaOH}$ and the final solution was allowed to stir for two days at room temperature. Excess salts were removed by dyalisis (against $\mathrm{H}_{2} \mathrm{O}$ ) for three days using a 1200014000 Da dialysis membrane.

Nuclear IMagnetic Resonance (NIMR) Characterization. All NMR experiments were performed on a Bruker Avance II NMR spectrometer operating at $600.16 \mathrm{MHz}$ for ${ }^{1} \mathrm{H}$ and $150.91 \mathrm{MHz}$ for ${ }^{13} \mathrm{C}$. Chemicals shifts are given in ppm using the residual solvent signal as reference. The multiplicity of the peaks is reported using the following abbreviations: $\mathrm{s}=$ singlet, $\mathrm{d}=$ doublet, $\mathrm{t}=$ triplet, $\mathrm{q}=$ quartet, $\mathrm{m}=$ multiplet. All $\mathrm{NMR}$ spectra were processed using TOPSPIN 2.1 and MestReC software packages. 
Mass Spectrometry (IMS). Mass spectra were acquired on a Waters Micromass LCT ESI mass spectrometer and a Waters MALDI Synapt mass spectrometer.

Flemental Analysis. Element analyses were done using an InterScience Flash 2000 organic elemental analyzer.

UV-Vis Spectroscopy. UV spectra were acquired on a Perkin Elmer Lambda 850 UV-Vis spectrometer using $1 \mathrm{~cm}$ quartz cuvettes.

Fluorescence Spectroscopy. All steady-state spectra were recorded using a Perkin Elmer Fluorescence Spectrometer.

Infrared Spectroscopy. All IR spectra were recorded using a Nicolet 6700/8700 Fourier Transform (FT-IR) spectrometer from Thermo Scientific. All samples (except AB-DO3A-SWCNTs) were measured using $\mathrm{KBr}$ pellets.

Vibrating Sample Magnetometry (VSIM). Magnetization of the different SWCNTs was measured using a 10 Vibrating Sample Magnetometer from Microsense.

\section{Transmission Flectron Microscopy (TEM) and Energy-Dispersive}

X-Ray (FDX). TEM images were obtained using analytical TEM FEI instruments. In order to prepare the samples, $10 \mu \mathrm{L}$ of the desired sample was applied onto Formvar-carbon coated grids (from electron microscopy sciences). After leaving the sample for 1 minute, the excess of liquid was removed using a piece of filter paper. Energy-dispersive X-ray (EDX) 
analysis was performed using a Thermo Noran System 6 with a resolution of $138 \mathrm{eV}$ on a Mn standard.

Magnetic Resonance Imaging (IMRI). All experiments were carried out on a 14.1 T (600 MHz) Avance II NMR Spectrometer from Bruker (Karlsruhe, Germany) equipped with a vertical narrow bore magnet (14.1 $T)$, a great $B_{0}$ compensation unit (BGU-II) and 3 great 1/60 amplifier units $(\mathrm{X}, \mathrm{Y}$ and $\mathrm{Z}$ ) also from Bruker. A micro 5 imaging probe, equipped with a $10 \mathrm{~mm}$ diameter saddle coil insert from Bruker (Karlsruhe, Germany) was used. The maximum gradient strength was $4.8 \mathrm{G} / \mathrm{cm} / \mathrm{A}$ and the temperature inside the probe was kept at $\sim 25^{\circ} \mathrm{C}$ by a gradient watercooling unit BCU20 from Bruker. All NMR/MRI tubes (5/10 mm diameter) were purchased from Wilmad Labglass (New Jersey, U.S.A.). Glass capillary tubes (melting point tubes, inner diameter $1 \mathrm{~mm}$ ) used for MRI were purchased from VWR. $T_{1}$-weighted images were obtained using a spin echo sequence with the following parameters: TR $=800 \mathrm{~ms}$, $\mathrm{TE}=6 \mathrm{~ms}, 1$ axial slice of $6 \mathrm{~mm}$ thickness, FOV $=10 \mathrm{~mm} \times 10 \mathrm{~mm}$, matrix size $=256 \times 256$ and 10 averages. The measurements were performed in a $1 \mathrm{~cm}$ (outer diameter) glass tube as follows. A stock solution of the $\mathrm{Gd}^{3+}$ containing complex was diluted 2, 5 and 10 times. For each experiment, capillary tubes of $1 \mathrm{~mm}$ inner diameter were filled with these solutions, placed in a custom-made Kel-F capillary holder and subsequently introduced in the $1 \mathrm{~cm}$ tube for imaging. Images were analyzed using Paravision 4.0 software.

\subsection{References}

(1) Boswell, C. A.; Eck, P. K.; Regino, C. A. S.; Bernardo, M.; Wong, K. J.; Milenic, D. E.; Choyke, P. L.; Brechbiel, M. W. Mol. Pharm. 2008, 5, 527. 
(2) Mamedov, I.; Parac-Vogt, T. N.; Logothetis, N. K.; Angelovski, G. Dalton Trans. 2010, 39, 5721.

(3) Manning, H. C.; Goebel, T.; Thompson, R. C.; Price, R. R.; Lee, H.; Bornhop, D. J. Bioconjugate Chem. 2004, 15, 1488.

(4) Mindt, T. L.; Müller, C.; Stuker, F.; Salazar, J.-F. d. r.; Hohn, A.; Mueggler, T.; Rudin, M.; Schibli, R. Bioconjug Chem 2009, 20, 1940.

(5) Kamaly, N.; Kalber, T.; Ahmad, A.; Oliver, M. H.; So, P.-W.; Herlihy, A. H.; Bell, J. D.; Jorgensen, M. R.; Miller, A. D. Bioconjugate Chem. 2008, 19, 118.

(6) Mulder, W. J. M.; Koole, R.; Brandwijk, R. J.; Storm, G.; Chin, P. T. K.; Strijkers, G. J.; deMelloDonega, C.; Nicolay, K.; Griffioen, A. W. Nano Lett. 2006, 6, 1.

(7) Mulder, W. J. M.; Strijkers, G. J.; Griffioen, A. W.; van Bloois, L.; Molema, G.; Storm, G.; Koning, G. A.; Nicolay, K. Bioconjugate Chem. 2004, 15, 799.

(8) Mulder, W. J. M.; Strijkers, G. J.; van Tilborg, G. A. F.; Cormode, D. P.; Fayad, Z. A.; Nicolay, K. Acc. Chem. Res. 2009, 42, 904.

(9) Pokorski, J. K.; Breitenkamp, K.; Liepold, L. O.; Qazi, S.; Finn, M. G. J. Am. Chem. Soc. 2011, 133, 9242.

(10) Song, Y.; Xu, X.; MacRenaris, K. W.; Zhang, X.-Q.; Mirkin, C. A.; Meade, T. J. Angew. Chem. 2009, 48, 9143.

(11) Yang, H.; Santra, S.; Walter, G. A.; Holloway, P. H. Adv. Mater. 2006, 18, 2890.

(12) Cotton, S. Lanthanide and Actinide Chemistry; John Wiley \& Sons, Ltd, 2006.

(13) Bottrill, M.; Kwok, L.; Long, N. J. Chem. Soc. Rev. 2006, 35, 557.

(14) Caravan, P.; Ellison, J. J.; McMurry, T. J.; Lauffer, R. B. Chem. Rev. 1999, 99, 2293.

(15) Reichert, D. E.; Lewis, J. S.; Anderson, C. J. Coord. Chem. Rev. 1999, $184,3$.

(16) Bunzli, J.-C. G.; Piguet, C. Chem. Soc. Rev. 2005, 34, 1048.

(17) Faulkner, S.; Pope, S. J. A.; Burton-Pye, B. P. Appl. Spectrosc. Rev. 2005, 40,1

(18) Beeby, A.; Bushby, L. M.; Maffeo, D.; Gareth Williams, J. A. J. Chem. Soc., Dalton Trans. 2002, 48.

(19) Terai, T.; Kikuchi, K.; Iwasawa, S.; Kawabe, T.; Hirata, Y.; Urano, Y.; Nagano, T. J. Am. Chem. Soc. 2006, 128, 8699.

(20) Faulkner, S.; Carrie, M.-C.; Pope, S. J. A.; Squire, J.; Beeby, A.; Sammes, P. G. Journal of the Chemical Society, Dalton Transactions 2004, 1405.

(21) Pal, R.; Parker, D. Org. Biomol. Chem. 2008, 6.

(22) Burton-Pye, B. P.; Heath, S. L.; Faulkner, S. Journal of the Chemical Society, Dalton Transactions 2005, 146.

(23) Koullourou, T.; Natrajan, L. S.; Bhavsar, H.; Pope, S. J. A.; Feng, J.; Narvainen, J.; Shaw, R.; Scales, E.; Kauppinen, R.; Kenwright, A. M.; Faulkner, S. J. Am. Chem. Soc. 2008, 130, 2178.

(24) Imbert, D.; Cantuel, M.; Bünzli, J.-C. G.; Bernardinelli, G.; Piguet, C. J. Am. Chem. Soc. 2003, 125, 15698. 
(25) Klink, S. I.; Keizer, H.; Veggel, F. C. J. M., van Angew. Chem., Int. Ed. 2000, 39, 4319.

(26) Coppo, P.; Duati, M.; Kozhevnikov, V. N.; Hofstraat, J. W.; De Cola, L. Angew. Chem., Int. Ed. 2005, 44, 1806.

(27) Andrews, M.; Amoroso, A. J.; Harding, L. P.; Pope, S. J. A. Dalton Trans. 2010, 39, 3407.

(28) Geninatti Crich, S. G.; Biancone, L.; Cantaluppi, V.; Esposito, D. D. G.; Russo, S.; Camussi, G.; Aime, S. Magn. Reson. Med. 2004, 51, 938.

(29) Pellegatti, L.; Zhang, J.; Drahos, B.; Villette, S.; Suzenet, F.; Guillaumet, G.; Petoud, S.; Toth, E. Chem. Commun. 2008, 6591.

(30) Picard, C.; Geum, N.; Nasso, I.; Mestre, B.; Tisnes, P.; Laurent, S.; Muller, R. N.; Vander Elst, L. Bioorg. Med. Chem. Lett. 2006, 16, 5309.

(31) Harte, A. J.; Jensen, P.; Plush, S. E.; Kruger, P. E.; Gunnlaugsson, T. Inorg. Chem. 2006, 45, 9465.

(32) Plush, S. E.; Gunnlaugsson, T. Dalton Trans. 2008, 3801.

(33) Gassensmith, J. J.; Arunkumar, E.; Barr, L.; Baumes, J. M.; DiVittorio, K. M.; Johnson, J. R.; Noll, B. C.; Smith, B. D. J. Am. Chem. Soc. 2007, 129, 15054.

(34) Gunnlaugsson, T.; Davis, A. P.; O'Brien, J. E.; Glynn, M. Org. Biomol. Chem. 2005, 3, 48.

(35) Kovacs, Z.; Sherry, A. D. Synthesis 1997, 7, 759.

(36) Osawa, M.; Hoshino, M.; Wada, T.; Hayashi, F.; Osanai, S. J. Phys. Chem. A 2009, 113, 10895.

(37) Borel, A.; Bean, J. F.; Clarkson, R. B.; Helm, L.; Moriggi, L.; Sherry, A. D.; Woods, M. Chemistry - A European Journal 2008, 14, 2658.

(38) Karousis, N.; Tagmatarchis, N.; Tasis, D. Chem. Rev. 2010, 110, 5366.

(39) Dumortier, H.; Lacotte, S.; Pastorin, G.; Marega, R.; Wu, W.; Bonifazi, D.; Briand, J. P.; Prato, M.; Muller, S.; Bianco, A. Nano Lett. 2006, 6, 1522.

(40) Kostarelos, K.; Lacerda, L.; Pastorin, G.; Wu, W.; Wieckowski, S.; Luangsivilay, J.; Godefroy, S.; Pantarotto, D.; Briand, J.-P.; Muller, S.; Prato, M.; Bianco, A. Nat. Nanotechnol. 2007, 2, 108.

(41) Wu, H.-C.; Chang, X.; Liu, L.; Zhao, F.; Zhao, Y. J. Mater. Chem. 2010, 20, 1036.

(42) Li, Y.; Zhang, X.; Luo, J.; Huang, W.; Cheng, J.; Luo, Z.; Li, T.; Liu, F.; Xu, G.; Ke, X.; Li, L.; Geise, H. J. Nanotechnology 2004, 15, 1645.

(43) Hartman, K. B.; Laus, S.; Bolskar, R. D.; Muthupillai, R.; Helm, L.; Toth, E.; Merbach, A. E.; Wilson, L. J. Nano Lett. 2008, 8, 415.

(44) Richard, C.; Doan, B. T.; Beloeil, J. C.; Bessodes, M.; Toth, E.; Scherman, D. Nano Lett. 2008, 8, 232.

(45) Zhang, J.; Zou, H.; Qing, Q.; Yang, Y.; Li, Q.; Liu, Z.; Guo, X.; Du, Z. J. Phys. Chem. B 2003, 107, 3712.

(46) Banin, U.; Millo, O. In Nanoparticles: from Theory to Application; Schmid, G., Ed.; Wiley-VCH Verlag GmbH \& Co. KGaA: Weinheim, FRG, 2005, p 305.

(47) Giljohann, D. A.; Seferos, D. S.; Daniel, W. L.; Massich, M. D.; Patel, P. C.; Mirkin, C. A. Angew. Chem., Int. Ed. 2010, 49, 3280.

(48) Hou, Y.; Hao, R. In Nanoplatform-Based Molecular Imaging; Chen, X., Ed.; John Wiley \& Sons, Inc.: 2011, p 529.

(49) Huang, W.-Y.; Davis, J. J. Dalton Trans. 2011, 40, 6087. 
(50) Manohar, S.; Ungureanu, C.; Van Leeuwen, T. G. Contrast Media Mol. Imaging 2011, 6, 389.

(51) Alric, C.; Taleb, J.; Duc, G. L.; Mandon, C.; Billotey, C.; Meur-Herland, A. L.; Brochard, T.; Vocanson, F.; Janier, M.; Perriat, P.; Roux, S.; Tillement, O. J. Am. Chem. Soc. 2008, 130, 5908.

(52) Sun, H.; Yuan, Q.; Zhang, B.; Ai, K.; Zhang, P.; Lu, L. Nanoscale 2011, 3, 1990.

(53) Judenhofer, M. S.; Wehrl, H. F.; Newport, D. F.; Catana, C.; Siegel, S. B.; Becker, M.; Thielscher, A.; Kneilling, M.; Lichy, M. P.; Eichner, M.; Klingel, K.; Reischl, G.; Widmaier, S.; Rocken, M.; Nutt, R. E.; Machulla, H.-J.; Uludag, K.; Cherry, S. R.; Claussen, C. D.; Pichler, B. J. Nat. Med. 2008, 14, 459.

(54) Cai, W.; Chen, K.; Li, Z.-B.; Gambhir, S. S.; Chen, X. J. Nucl. Med. 2007, 48, 1862.

(55) Catana, C.; Wu, Y.; Judenhofer, M. S.; Qi, J.; Pichler, B. J.; Cherry, S. R. J. Nucl. Med. 2006, 47, 1968.

(56) Cherry, S. R.; Louie, A. Y.; Jacobs, R. E. P. IEEE 2008, 96, 416.

(57) Choi, J. S.; Park, J. C.; Nah, H.; Woo, S.; Oh, J.; Kim, K. M.; Cheon, G. J.; Chang, Y.; Yoo, J.; Cheon, J. Angew. Chem., Int. Ed. 2008, 47, 6259.

(58) Culver, J.; Akers, W.; Achilefu, S. J. Nucl. Med. 2008, 49, 169.

(59) Morawski, A. M.; Lanza, G. A.; Wickline, S. A. Curr. Opin. Biotechnol. 2005, 16, 89.

(60) Kim, E. E. Korean J. Radiol. 2003, 4, 201.

(61) Accardo, A.; Tesauro, D.; Aloj, L.; Pedone, C.; Morelli, G. Coord. Chem. Rev. 2009, 253, 2193.

(62) Torchilin, V. P. AAPS J. 2007, 9, E128.

(63) Overoye-Chan, K.; Koerner, S.; Looby, R. J.; Kolodziej, A. F.; Zech, S. G.; Deng, Q.; Chasse, J. M.; McMurry, T. J.; Caravan, P. J. Am. Chem. Soc. 2008, 130, 6025.

(64) Ye, F.; Jeong, E.-K.; Jia, Z.; Yang, T.; Parker, D.; Lu, Z.-R. Bioconjugate Chem. 2008, 19, 2300.

(65) Caravan, P. Acc. Chem. Res. 2009, 42, 851.

(66) Xu, H.; Regino, C. A. S.; Koyama, Y.; Hama, Y.; Gunn, A. J.; Bernardo, M.; Kobayashi, H.; Choyke, P. L.; Brechbiel, M. W. Bioconjugate Chem. 2007, 18, 1474.

(67) van Tilborg, G. A. F.; Mulder, W. J. M.; Chin, P. T. K.; Storm, G.; Reutelingsperger, C. P.; Nicolay, K.; Strijkers, G. J. Bioconjugate Chem. 2006, 17, 865.

(68) Prinzen, L.; Miserus, R.-J. J. H. M.; Dirksen, A.; Hackeng, T. M.; Deckers, N.; Bitsch, N. J.; Megens; Douma, K.; Heemskerk, J. W.; Kooi, M. E.; Frederik, P. M.; Slaaf, D. W.; van Zandvoort, M. A. M. J.; Reutelingsperger, C. P. M. Nano Lett. 2006, 7, 93.

(69) Li, H.; Gray, B. D.; Corbin, I.; Lebherz, C.; Choi, H.; Lund-Katz, S.; Wilson, J. M.; Glickson, J. D.; Zhou, R. Acad. Radiol. 2004, 11, 1251.

(70) Edwards, W. B.; Xu, B.; Akers, W.; Cheney, P. P.; Liang, K.; Rogers, B. E.; Anderson, C. J.; Achilefu, S. Bioconjugate Chem. 2007, 19, 192.

(71) Kelkar, S. S.; Reineke, T. M. Bioconjugate Chem. 2011, 22, 1879. 
(72) Kim, J.; Piao, Y.; Hyeon, T. Chem. Soc. Rev. 2009, 38, 372.

(73) Block, E.; Eswarakrishnan, V.; Gernon, M.; Ofori-Okai, G.; Saha, C.; Tang, K.; Zubieta, J. J. Am. Chem. Soc. 1989, 111, 658.

(74) Vetterlein, K.; Bergmann, U.; Büche, K.; Walker, M.; Lehmann, J.; Linscheid, M. W.; Scriba, G. K. E.; Hildebrand, M. Electrophoresis 2007, 28, 3088. 


\section{Appendix 6.1. Characterization of the Building Blocks 3 and 8}

Owing to the symmetry of the propyl alkylated anthracene (2) (Figure 6.11, top), a single set of ${ }^{1} \mathrm{H}$ NMR peaks were observed. Upon alkylation of the maleimide with the propyl alkylated anthracene core (2), a shift in all proton signals was observed. As expected, all the signals corresponding to the propyl alkyl linker (a,b and $\mathbf{c}$ ) are downfield shifted, with a (corresponding to the methylene closest to the substituted group) showing the largest shift. This observation indicates changes in the chemical environment due to the presence of electron-withdrawing functional groups such as maleimides. Moreover, splitting of $\mathbf{a}, \mathbf{b}$ and $\mathbf{c}$ into two identical, overlapping peaks was observed; thereby suggesting that (for each set of protons) full equivalency was lost. Despite various purification attempts, several small signals (labeled as a', b' and c'), were also observed. These signals could arise from either as impurities (as elemental analysis showed a deviation of $6 \%$ ) or as conformers. However, the latter statement is contradicted by ${ }^{1} \mathrm{H}$ NMR data obtained at $50^{\circ} \mathrm{C}$ (Figure 6.11 , bottom), which shows that these signals (a', b' and c') do not shift after increasing the temperature. 


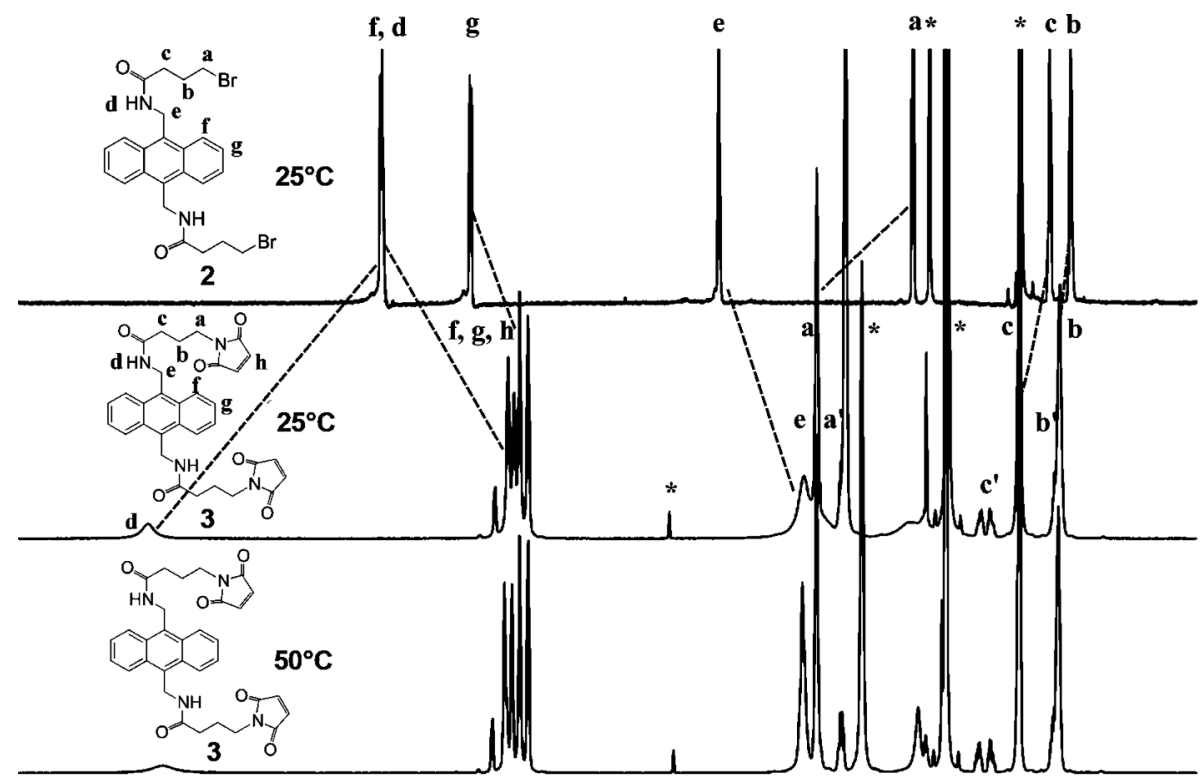

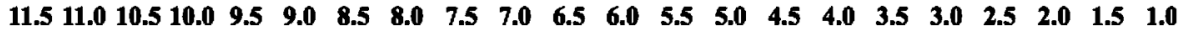
$\delta$ (ppm)

Figure 6.11. ${ }^{1} \mathrm{H}$ NMR spectra of 2 and $3\left(\right.$ at $25^{\circ} \mathrm{C}$ and $50^{\circ} \mathrm{C}$ ) in $\mathrm{d}_{6}$-DMSO. Residual solvents are indicated with an asterisk $\left({ }^{*}\right)$.

${ }^{1} \mathrm{H}$ NMR spectra at $50^{\circ} \mathrm{C}$ also revealed that the rotational freedom around e (methylene protons connecting the amide to the anthracene) was restricted after the incorporation of the maleimide (3). This is illustrated by the broadening of this signal (e) after the maleimide alkylation (Figure 6.11, top and center), followed by sharpening as the temperature was increased (Figure 6.11, bottom). This restricted rotational motion, together with the shifting to higher field of the aromatic protons $\mathbf{f}$ and $\mathbf{g}$ strongly suggest a "rigid" conformation most likely resulting from $\pi$-stacking of the maleimide with the anthracene. The second building block used for the synthesis of the ANT-2DO3A ligand (8, Scheme 6.2) was successfully characterized using the above-mentioned techniques. 
CHAPTER 6 


\section{SUMMARY}

Molecular imaging is a discipline in charge of the rapid and accurate visualization of the human body in a, preferably, non-invasive way. Despite the wide variety of imaging modalities available today, precise differentiation of physiologically/pathologically different tissues remains a challenge. While the existing imaging agents (IAs), used to aid image contrast without perturbing metabolic processes, have contributed to a more accurate visualization using different molecular imaging modalities, shortcomings to be overcome still remain. Many of them are related to the well-known limited spatial resolution and/or low sensitivity that most imaging techniques suffer from (Figure 1).

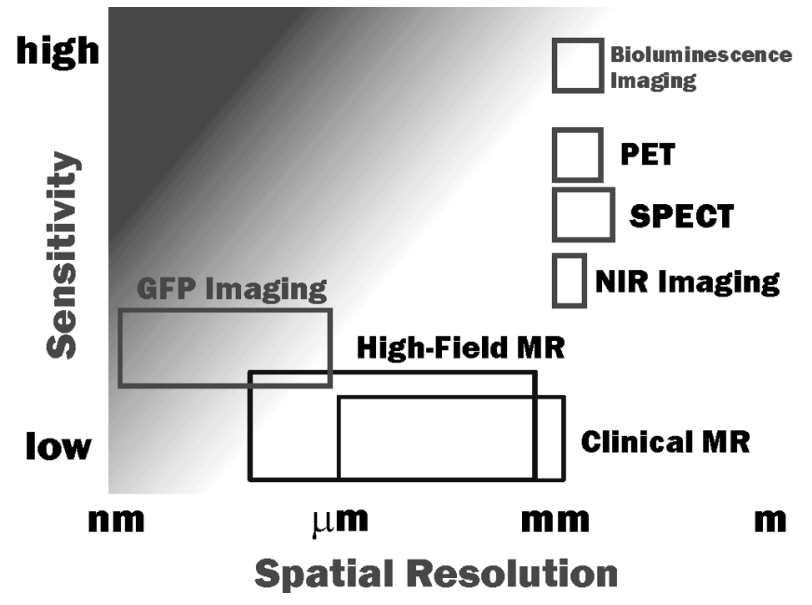

Figure 1. Sensitivity vs. spatial resolution of selected imaging modalities. PET: positron emission tomography. SPECT: single-photon emission computed tomography. NIR: nearinfrared. MR: magnetic resonance. GFP: green fluorescent protein. 
A common way to tackle with the low sensitivity and/or resolution issue is by combining two (or more) imaging modalities. This emerging field, known as multimodal imaging, is dedicated to the optimization of the synergy of different imaging modalities in order to combine "the best of both worlds". Despite the various strategies and combinations described in the literature, one of the most preferred ones regards the use of Gadolinium $\left(\mathrm{Gd}^{3+}\right)$ complexes for magnetic resonance imaging (MRI), combined with fluorescent probes for optical imaging. In this thesis, new approaches directed towards simple and functional IAs for MR and fluorescence multimodal imaging are proposed. The multimodal IAs described here are based on combinations of (novel) metal complex-based ligands and nanostructures, where the organic ligands not only serve as chelators for metal complexation, but also act as templates/scaffolds for the growth of nanostructures.

In Chapter 3, the aqueous-based, one-step synthesis of hybrid silver nanostructures (hAgNSs), grown using a polyamino carboxylic acid scaffold, namely 1,4,7,10-tetraaza-1-(1-aminobutyl)-4,7,10-triacetic acid cyclododecane (AB-DO3A, Figure 2A), is described. The nanostructures formed are characterized and a feasible arrangement for these hAgNSs is proposed using a wide range of techniques, including UV-Vis absorbance spectroscopy, transmission electron microscopy (TEM), dynamic light scattering (DLS), fluorescence spectroscopy (lifetime and steady-state), mass spectrometry (MS), NMR spectroscopy and infrared spectroscopy. The engineered $h$ AgNSs consist of two functional units: a "large" silver nanoparticle (AgNP, stabilized by the carboxylate moieties and the cyclen core of the AB-DO3A scaffold), which can act both as a template for surface modification and potentially as a therapeutic agent, and "smaller" 
Ag nanoclusters (AgNCs) that can be used as optical trackers due to their fluorescent properties (Figure 2B).

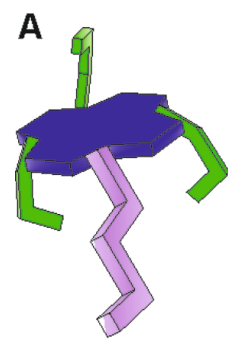

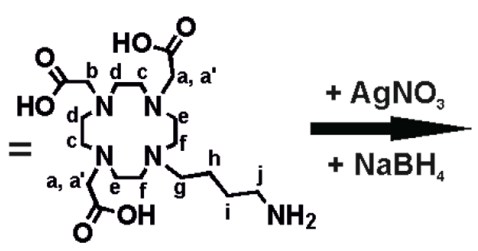

$A B-D O 3 A$

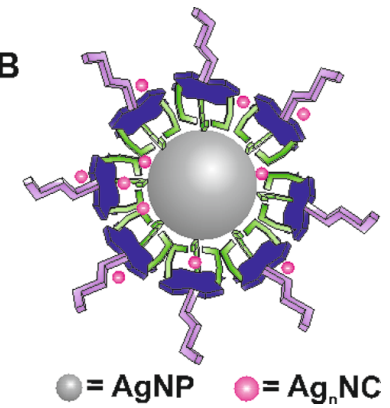

Figure 2. Hybrid silver nanostructures (hAgNSs), formed by the chemical reduction of $\mathrm{AgNO}_{3}$ in the presence of $\mathrm{AB}-\mathrm{DO} 3 \mathrm{~A}$. The localization of the $\mathrm{Ag}_{\mathrm{n}} \mathrm{NCs}$ within the AgNP is only a schematic representation.

The use of (similar) aggregated organic ligands as nucleation points for the formation of protein cages of the cowpea chlorotic mottle virus (CCMV) is described in Chapter 4. Stable assemblies are engineered by pure hierarchical self-assembly of micelles of the 1,4,7,10-tetraza-1-(1carboxymethylundecane)-4,7,10-triacetic acid cyclododecane (DOTAC10) ligand inside CCMV (Figure 3). The DOTAC10 ligand is used to either complex with $\mathrm{Gd}^{3+}$ in order to form paramagnetic micelles visualized by $\mathrm{MRI}$ or to encapsulate amphiphilic $\mathrm{Zn}^{2+}$ phthalocyanine $(\mathrm{ZnPc})$ dye molecules, in order to confirm (optically) the encapsulation of the micelles. Subsequent incorporation of the $\mathrm{ZnPc}$ dye in the $\mathrm{Gd}^{3+}$-DOTAC10 paramagnetic micelles leads to noticeably higher capsid loadings, as observed by $\mathrm{MRI}$ and elemental analysis. These purely self-assembled protein cages (as confirmed by TEM and size exclusion chromatography) show enhanced ionic and capsid $r_{1}$ relaxivities which are attributed to aggregation and altered micelle association kinetics resulting from local confinement inside the protein cage and $\mathrm{ZnPc}$ dye encapsulation. 


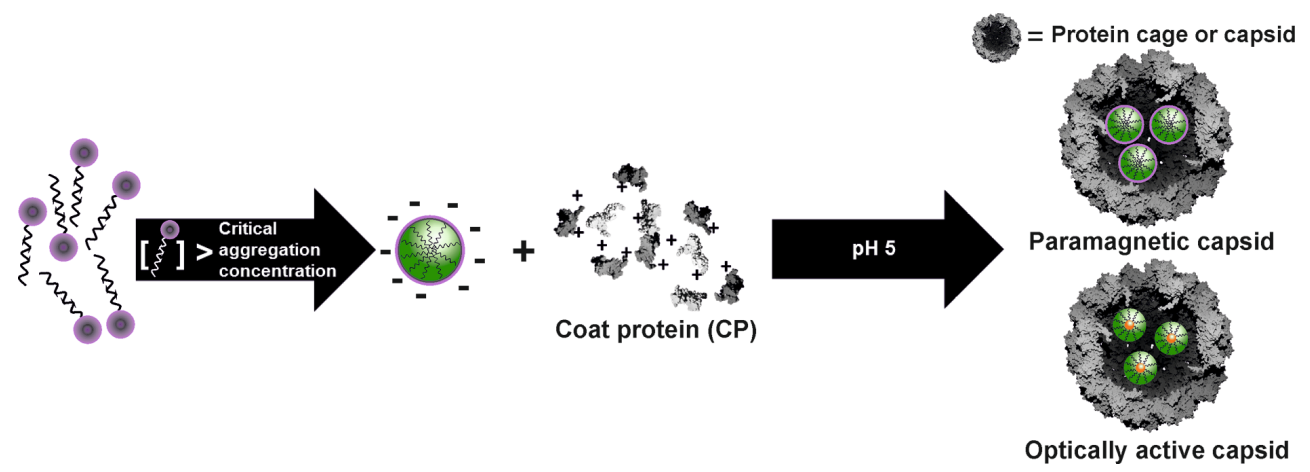

Figure 3. Schematic representation of the self-assembly triggered by self-assembly process: micelles are formed when the monomer concentration is higher than the critical aggregation concentration (cac). The negatively charged micelles act as "nucleation points" for self-assembly of CCMV coat proteins (CPs), yielding stable paramagnetic and optically active protein cages. The localization and orientation of the $\mathrm{ZnPc}$ molecules within the micelles is only schematic.

In Chapter 5, the synthesis, characterization and study of the cytotoxicity of multimodal gold nanorods (MMAuNRs) for magnetic resonance and optical imaging is described. The gold nanostructures are modified by thiol-based ligand exchange using an optimized O-[2-(3mercaptopropionylamino)ethyl]-O'-methylpolyethylene glycol (PEG-SH), $\mathrm{Gd}^{3+}$-1-(11-sulfanylundecyl)-4,7,10-tris(carboxymethyl)-1,4,7,10tetraazacyclododecane $\left(\mathrm{Gd}^{3+}-\mathrm{SUDDO} 3 \mathrm{~A}\right)$ and (3aS,4S,6aR)-11sulfanylundecyl 5-(hexahydro-2-oxo-1H-thieno[3,4-d]imidazol-4-yl) pentanoate (Biotin-SH) ratio. Subsequent incubation with Cy5-streptavidin leads to paramagnetic and fluorescent AuNRs, visualized by MRI and fluorescence microscopy (Figure 4) and characterized by means of UV-Vis absorption spectroscopy, TEM and total reflection $x$-ray fluorescence (TXRF). The MMAuNRs obtained using this one-pot synthesis are biocompatible, show median lethal concentration $\left(\mathrm{LC}_{50}\right)$ values $>150 \mathrm{pM}$ 
AuNR, improved relaxivity properties, high $\mathrm{Gd}^{3+}$ loadings and can be optically colocalized with mouse myoblast (C2C12) cells in vitro.

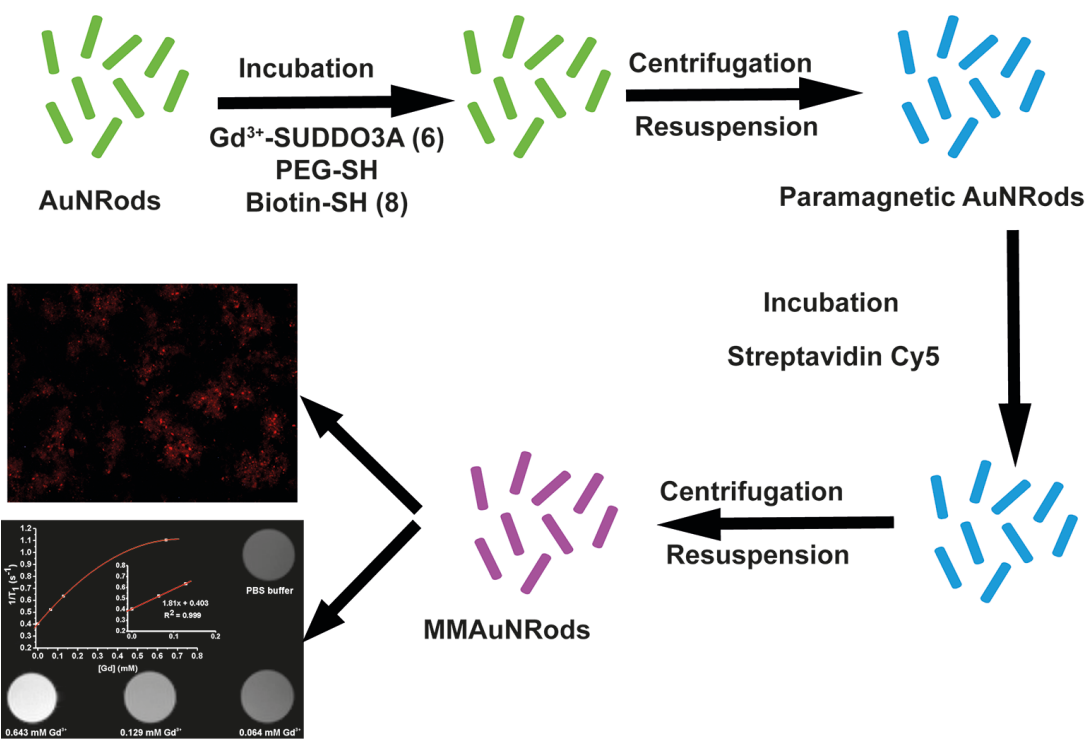

Figure 4. Schematic representation of the synthesis of MMAuNRs. (Upper) Fluorescence image: red emission observed corresponding to Cy5-labeled MMAuNRs (exposure time $2000 \mathrm{~ms}$ ). (Lower) MR Image: $T_{1}$-weighted MR image of MMAuNRs at different $\mathrm{Gd}^{3+}$ concentrations $(600 \mathrm{MHz}, \mathrm{TE}=4 \mathrm{~ms}, \mathrm{TR}=500 \mathrm{~ms})$. The plot shows ionic $r_{1}$ relaxivity corresponding to the MMAuNRs. The inset plot corresponds to a linear fitting of the $0-0.13$ $\mathrm{mM} \mathrm{Gd}^{3+}$ concentration range $\left(r_{1}=1.8 \mathrm{mM}^{-1} \mathrm{~s}^{-1}\right)$.

In Chapter 6, functionalized carbon-based organic scaffolds are proposed as platforms for multiple lanthanide complexation by integration of some of the metal complex-based templates described in previous chapters. First, a small, water-soluble carbon-based scaffold, consisting of an anthracene core (ANT) where two identical 1,4,7,10-tetraazacyclododecane-1,4,7triacetic acid (DO3A)-based chelators (similar to the one presented in Chapter 5) are integrated, is presented. The promising optical and paramagnetic properties observed for this water soluble ANT-2DO3A bismacrocyclic ligand (Figure 5) show its potential as a proof-of-principle 
probe for further development of small molecule, multimodal IAs by exploiting the chemically similar, but electronically/magnetically different, properties lanthanides show.
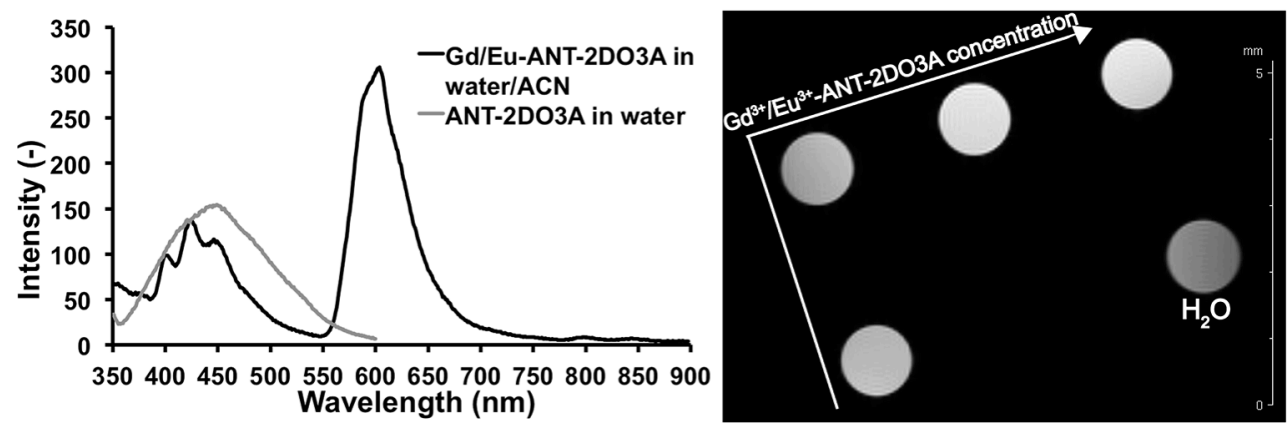

Figure 5. Left: emission spectra of ANT-2DO3A (grey line, $\mathrm{H}_{2} \mathrm{O}, \lambda_{\text {exc }}=312 \mathrm{~nm}$ ) and $\mathrm{Gd}^{3+} / \mathrm{Eu}^{3+}$-ANT-2DO3A (black line, $\mathrm{H}_{2} \mathrm{O} / \mathrm{ACN}, \lambda_{\text {exc }}=262 \mathrm{~nm}$ ). Right: $T_{1}$-weighted MR image showing various concentrations $(4,2,0.8$ and $0.4 \mathrm{~g} / \mathrm{L})$ of $\mathrm{Gd}^{3+} / \mathrm{Eu}^{3+}-\mathrm{ANT}-2 \mathrm{DO} 3 \mathrm{~A}$ complex and $\mathrm{H}_{2} \mathrm{O}$ (control).

Later, the covalent attachment of the AB-DO3A template (shown in Chapter 4) to carbon nanotubes (CNTs) is explored. CNTs are attractive platforms for the incorporation of large payloads of (various) molecular imaging probes due to their high surface-to-volume ratio. The covalent functionalization of SWCNTs with the AB-DO3A ligand, whose water solubility is enhanced by the three carboxylic acids of this ligand, followed by lanthanide complexation, leads to the formation of functionalized SWCNTS (Figure 6). 

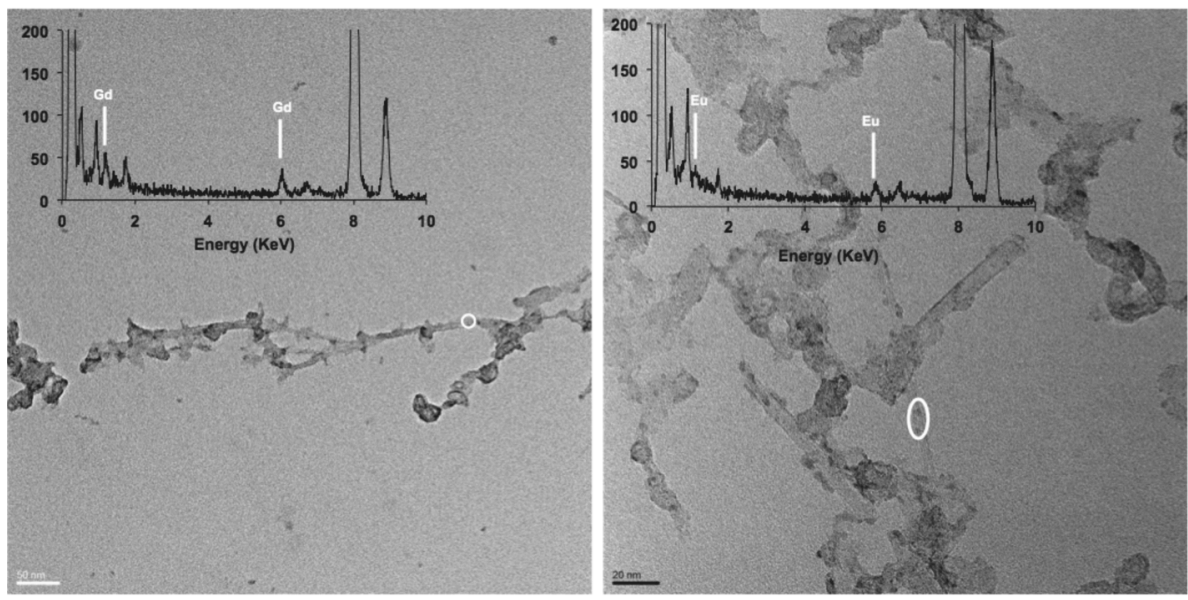

Figure 6. TEM-images of $\mathrm{Gd}^{3+}-\mathrm{AB}-\mathrm{DO} 3 \mathrm{~A}-\mathrm{SWCNTS}$ (left) and $\mathrm{Eu}^{3+}-\mathrm{AB}-\mathrm{DO} 3 \mathrm{~A}-\mathrm{SWCNTS}$ (right) (scale bar left $=50 \mathrm{~nm}$ and right $=20 \mathrm{~nm}$ ). Inset in both pictures is the EDX spectrum of the regions highlighted in white.

The preliminary characterization and evaluation of the properties of these functionalized carbon-based scaffolds show promising results, thereby highlighting their potential for multimodal imaging purposes. Finally, the future perspectives and challenges ahead for these metal complex-based templates, nanostructures and the multimodal IAs described in this thesis are briefly discussed. 


\section{SAMENVATTING}

Moleculaire beeldvorming is een discipline die verantwoordelijk is voor de snelle en nauwkeurige visualisatie van het menselijk lichaam middels een, bij voorkeur, niet-invasieve manier. Ondanks het aantal beeldvormende technieken die tegenwoordige beschikbaar zijn, blijft een nauwkeurige onderscheid tussen fysiologisch/pathologisch verschillende weefsels een uitdaging. Hoewel de bestaande beeldvormingsagenten (BAs), welke gebruikt worden om het contrast te verbeteren zonder daarbij de verschillende metabolische processen te verstoren, hebben bijgedragen aan een betere visualisatie zijn er nog beperkingen en tekortkomingen. Deze beperkingen zijn gerelateerd aan zaken als de beperkte resolutie en/of lage gevoeligheid van de meeste technieken (Figuur 1).

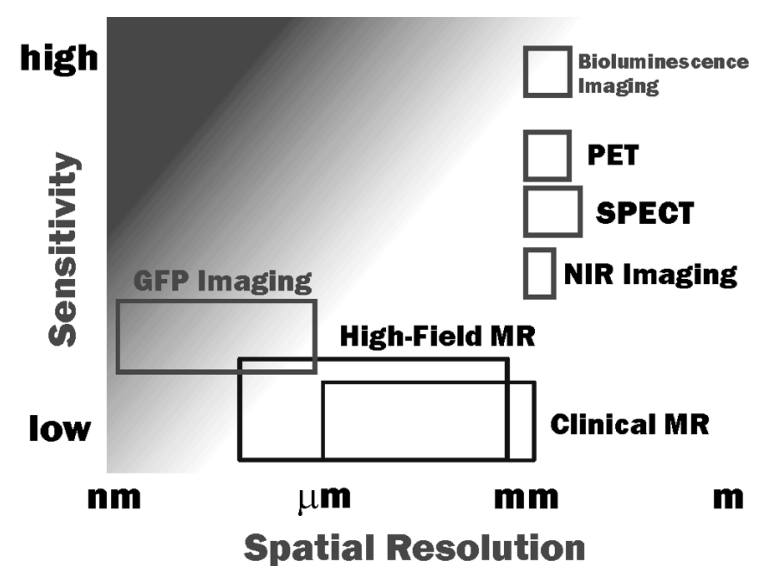

Figuur 1. Gevoeligheid vs. resolutie van geselecteerde beeldvormende technieken. PET: positronenemissietomografie. SPECT: single-photon emission computed tomography. NIR: nabij-infrarood. MR: magnetische resonantie. GFP: groen fluorescent proteïne. 
Een veel voorkomende manier om de lage gevoeligheid en/of resolutie kwestie te verbeteren is door het combineren van twee (of meer) beeldvormende technieken. Dit nieuwe begrip, bekend als multimodale beeldvorming, behelst het optimaliseren van de synergie tussen verschillende beeldvormende technieken met als doel om "het beste van beide werelden" te verenigen. Van de in literatuur beschreven veelheid aan strategieën en combinaties, is (één van) de meest voorkomende multimodale beeldvormingsmethode(s) het gebruik van Gadolinium ( $\mathrm{Gd}^{3+}$ ) complexen (voor beeldvorming met magnetische resonantie, MRI) in combinatie fluorescerende probes (voor optische beeldvorming). In dit proefschrift worden nieuwe multimodale beeldvormingsbenaderingen beschreven die gericht zijn op eenvoudige en functionele BAs voor MR en fluorescentie beeldvorming. De beschreven multimodale BAs zijn gebaseerd op combinaties van (nieuwe) metaalcomplex-gebaseerde liganden en nanostructuren, waarbij de organische liganden niet alleen als chelaten voor metaalcomplexvorming worden gebruikt, maar ook als malplaatje/bouwsteiger voor de groei van nanostructuren.

In hoofdstuk 3 wordt de water-gebaseerde synthese van hybride zilver nanostructuren (hAgNSs) gegroeid met een polyamino carbonzuur bouwsteiger, te weten 1,4,7,10-tetraaza-1-(1-aminobutyl)-4,7,10-triacetic acid cyclododecane (AB-DO3A, Figuur 2A), beschreven. De gevormde nanostructuren zijn gekarakteriseerd en een mogelijke structuur voor deze hAgNSs is voorgesteld op basis van verschillende analyse technieken, waaronder UV-Vis absorptie spectroscopie, transmissieelektronenmicroscopie (TEM), dynamische lichtverstrooiing (DLS), fluorescentie spectroscopie (levensduur en steady-state), massaspectrometrie (MS), kernspinresonantie (NMR) en infrarood spectroscopie. De gevormde hAgNSs bestaan uit twee functionele 
eenheden: een "groot" zilver nanodeeltje (AgNP, gestabiliseerd door de carboxylaat groepen en cyclen kern van de AB-DO3A bouwsteiger), die kan dienen als zowel malplaatje voor oppervlakte modificatie alsmede als therapeutisch middel, en "kleinere" Ag nanoclusters (AgNCs) die gebruikt kunnen worden als optische "trackers" door hun fluorescerende eigenschappen (Figuur 2B).

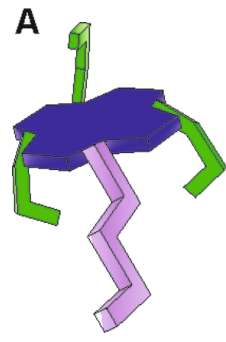

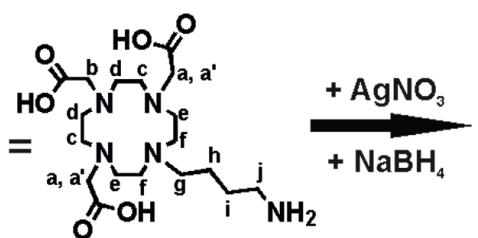

AB-DO3A

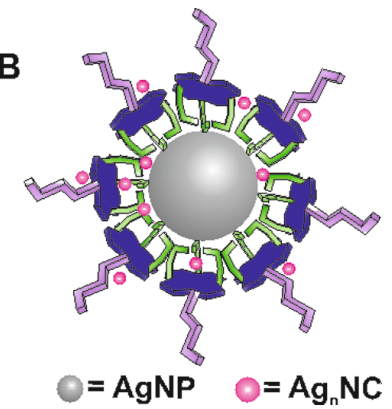

Figuur 2. Hybride zilver nanostructuren (hAgNSs), gevormd door de chemische reductie van $\mathrm{AgNO}_{3}$ in aanwezigheid van $\mathrm{AB}-\mathrm{DO} 3 \mathrm{~A}$. De lokalisatie van de $\mathrm{Ag}_{\mathrm{n}} \mathrm{NCs}$ in de $\mathrm{AgNP}$ is slechts schematisch weergeven.

Het gebruik van (soortgelijke) geaggregeerde organische liganden als nucleatiepunten voor de vorming van eiwitkooien van het cowpea chlorotic mottle virus (CCMV) wordt beschreven in Hoofdstuk 4. Stabiele assemblages zijn ontwikkeld door puur hiërarchische zelf-assemblage van micellen van het 1,4,7,10-tetraza-1-(1-carboxymethylundecane)-4,7,10triacetic acid cyclododecane (DOTAC10) ligand binnen CCMV (Figuur 3). Het DOTAC10 ligand is gebruikt voor complexatie met $\mathrm{Gd}^{3+}$ om paramagnetische micellen te vormen welke gevisualiseerd zijn met MRI, of om amfifiele $\mathrm{Zn}^{2+}$ phthalocyanine ( $\mathrm{ZnPc}$ ) kleurstofmoleculen in te kapselen, die gebruikt zijn om de inkapseling van de micellen optisch te verifiëren. Opname van $\mathrm{ZnPc}$ in de $\mathrm{Gd}^{3+}$-DOTAC10 paramagnetische micellen leidt tot aanzienlijk hogere inkapseling, zoals geanalyseerd met MRI en 
elementaire analyse. De zuivere zelf-geassembleerde eiwitkooien (bevestigd door TEM en size exclusion chromatografie) hebben verbeterde $r_{1}$ relaxatietijden die toegewezen worden aan de aggregatie en veranderde micel kinetiek als gevolg van lokale opsluiting in de eiwitkooi en de $\mathrm{ZnPc}$ kleurstof inkapseling.

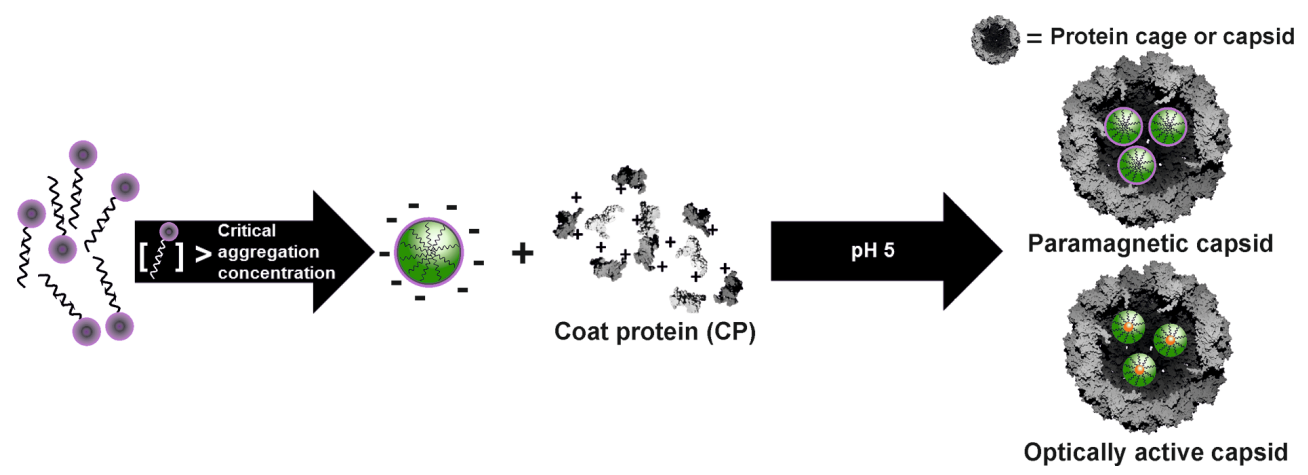

Figuur 3. Schematische weergave van het "zelf-assemblage geactiveerd door zelfassemblage" proces: micellen worden gevormd wanneer de monomeer concentratie hoger is dan de kritische aggregatie concentratie (cac). De negatief geladen micellen dienen als "nucleatiepunten" voor zelf-assemblage van CCMV eiwitten (CP), wat stabiele paramagnetische en optisch actieve eiwit kooien oplevert. De lokalisatie en oriëntatie van de moleculen in de ZnPc micellen is slechts schematisch.

In hoofdstuk 5 wordt de synthese, karakterisatie en toxiciteitsstudie beschreven van multimodale goud nanostaafjes (MMAuNRs) voor magnetische resonantie en optische beeldvorming. De goud nanostructuren zijn gemodificeerd middels thiol-gebaseerde liganduitwisseling met een geoptimaliseerde verhouding van O-[2-(3mercaptopropionylamino)ethyl]-O'-methylpolyethylene glycol (PEG-SH), $\mathrm{Gd}^{3+}$-1-(11-sulfanylundecyl)-4,7,10-tris(carboxymethyl)-1,4,7,10tetraazacyclododecane $\left(\mathrm{Gd}^{3+}-\mathrm{SUDDO} \mathrm{A}\right)$ en $(3 \mathrm{aS}, 4 \mathrm{~S}, 6 \mathrm{aR})-11-$ sulfanylundecyl 5-(hexahydro-2-oxo-1H-thieno[3,4-d]imidazol-4-yl) pentanoate (Biotin-SH). Incubatie met Cy5-streptavidine resulteert in 
paramagnetische en fluorescerende AuNRs, welke zijn gevisualiseerd met MRI en fluorescentie microscopie (Figuur 4) en gekarakteriseerd door middel van UV-Vis absorptie spectroscopie, TEM en totale reflectie X-ray fluorescentie (TXRF). De MMAuNRs, verkregen met behulp van deze "onepot" synthese, zijn biocompatibel, hebben median lethal concentration $\left(\mathrm{LC}_{50}\right)$ waarden > 150 pM AuNR, verbeterde relaxatie eigenschappen, hogere $\mathrm{Gd}^{3+}$ hoeveelheden en kunnen optisch worden "colocalized" met muis myoblast $(\mathrm{C} 2 \mathrm{C} 12)$ cellen in vitro.

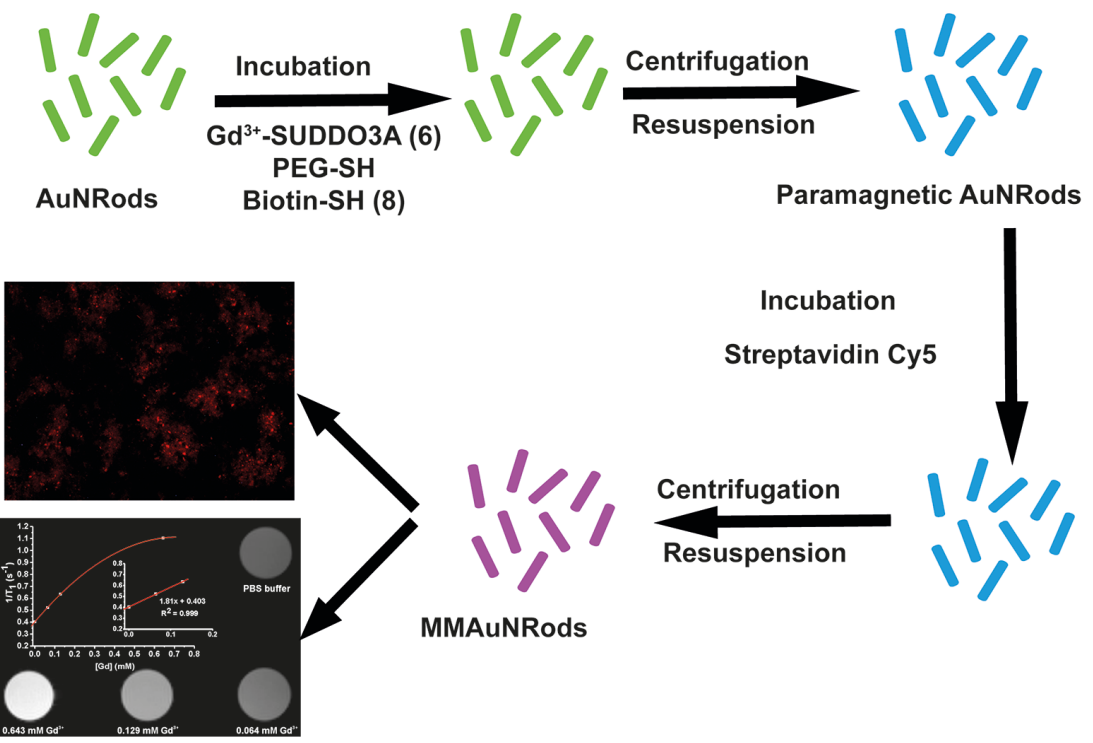

Figuur 4. Schematische weergave van de synthese van MMAuNRs. (Bovenste) Fluorescentie beeld: de waargenomen rode emissie komt overeen met Cy5-gelabelde MMAuNRs (belichtingstijd $2000 \mathrm{~ms}$ ). (Onderste) MR Afbeelding: $T_{1}$-gewogen MR afbeelding van MMAuNRs bij verschillende $\mathrm{Gd}^{3+}$ concentraties $(600 \mathrm{MHz}, \mathrm{TE}=4 \mathrm{~ms}$, TR $=500 \mathrm{~ms})$. De grafiek toont de ionische $r_{1}$ relaxatietijd die hoort bij de MMAuNRs. De inzet-plot correspondeert met de raaklijn van het $0-0.13 \mathrm{mM} \mathrm{Gd}^{3+}$ concentratiebereik $\left(r_{1}=1.8 \mathrm{mM}^{-1} \mathrm{~s}^{-1}\right)$.

In hoofdstuk 6 worden gefunctionaliseerde koolstof-gebaseerde organische bouwsteigers voorgesteld als platforms voor (meerdere) 
lanthanide complexatie(s) door integratie van een aantal van de metaalcomplex-gebaseerde malplaatjes beschreven in voorgaande hoofdstukken. Als eerste wordt een kleine, water oplosbare koolstofgebaseerde bouwsteiger beschreven, bestaande uit een anthraceen (ANT) kern waarin twee identieke 1,4,7,10-tetraazacyclododecane-1,4,7-triacetic acid (DO3A)-based liganden (vergelijkbaar met die beschreven in hoofdstuk 5) zijn geïntegreerd. De veelbelovende optische en paramagnetische eigenschappen voor het water oplosbare ANT-2DO3A bis-macrocyclische ligand (Figuur 5) laten het potentieel zien als proof-ofprinciple probe en als basis dienen voor de verdere ontwikkeling van kleine, multimodale BAs door gebruik te maken van de chemisch vergelijkbare, maar toch elektronisch/magnetisch verschillende, eigenschappen die lanthaniden bezitten.
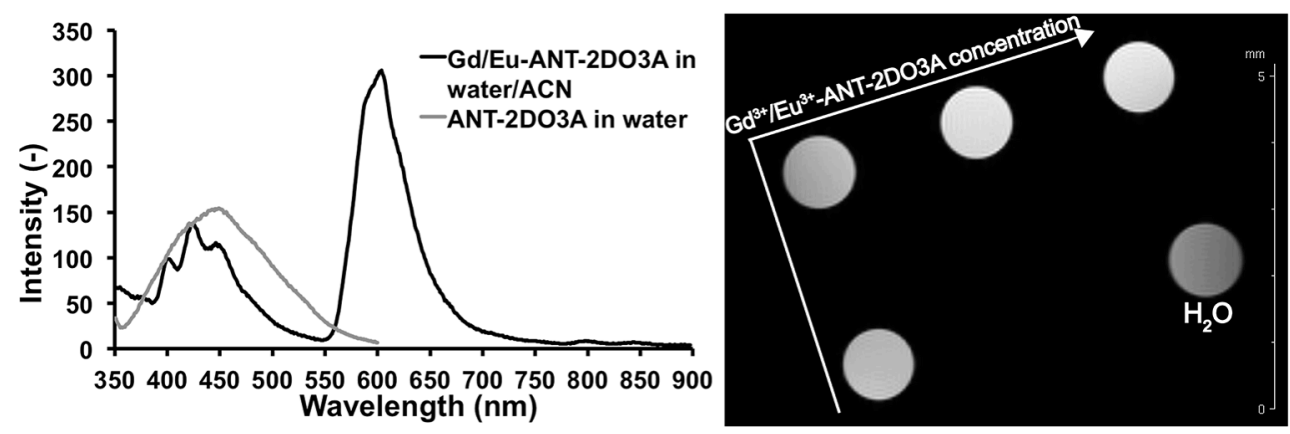

Figuur 5. Links: emissiespectra van ANT-2DO3A (grijze lijn, $\mathrm{H}_{2} \mathrm{O}, \quad \lambda_{\text {exc }}=312 \mathrm{~nm}$ ) en $\mathrm{Gd}^{3+} / \mathrm{Eu}^{3+}$-ANT-2DO3A (zwarte lijn, $\mathrm{H}_{2} \mathrm{O} / \mathrm{ACN}, \lambda_{\text {exc }}=262 \mathrm{~nm}$ ). Rechts: $T_{1}$-gewogen MR afbeelding met verschillende concentraties $(4,2,0,8$ en $0,4 \mathrm{~g} / \mathrm{L})$ van $\mathrm{Gd}^{3+} / \mathrm{Eu}^{3+}-\mathrm{ANT}-2 \mathrm{DO} \mathrm{A}$ complex en $\mathrm{H}_{2} \mathrm{O}$ (controle).

Vervolgens is de covalente binding van het $A B-D O 3 A$ malplaatje (zie hoofdstuk 4) aan koolstofnanobuisjes (CNTs) onderzocht. Enkelwandige CNTs (SWCNTs) zijn aantrekkelijke platforms voor het bevestigen van grote hoeveelheden van (verschillende) moleculaire BAs vanwege hun 218 
hoge oppervlakte-volumeverhouding. De covalente functionalisering van SWCNTs met het AB-DO3A ligand, welke de wateroplosbaarheid van de CNTs verhoogt door de drie carbonzuren van dit ligand, gevolgd door lanthanide complexatie, leidt tot de vorming van gefunctionaliseerde SWCNTs (Figuur 6).
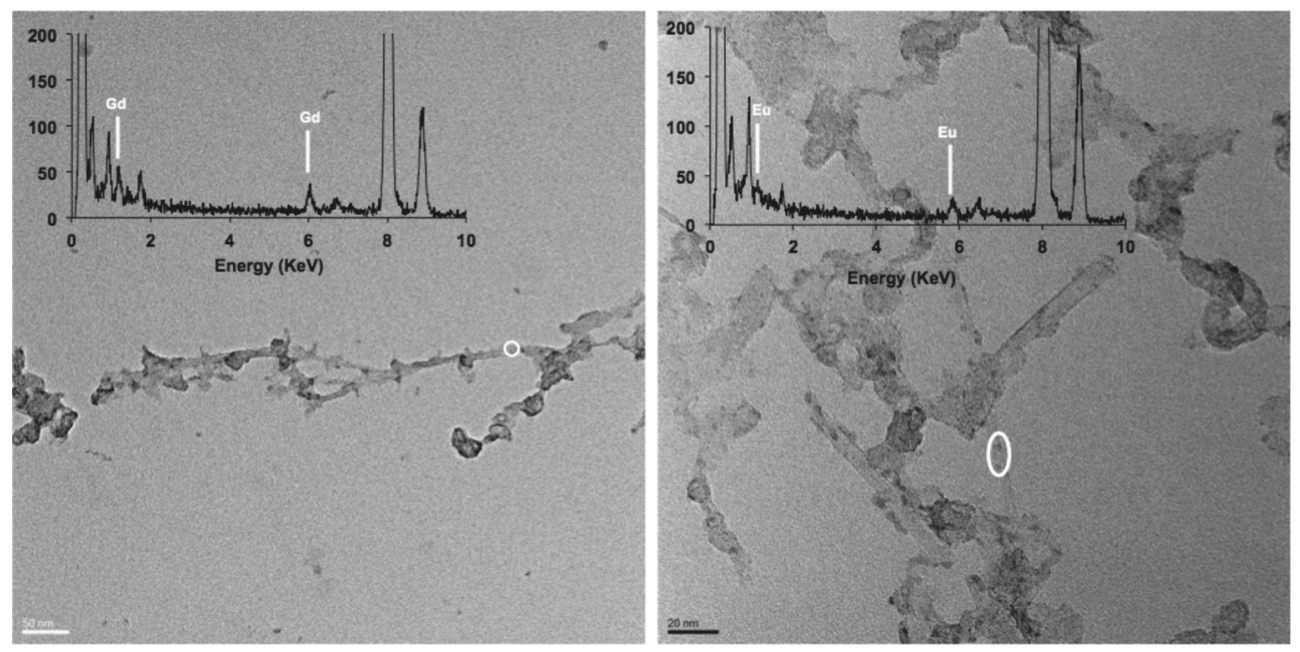

Figuur 6. TEM-beelden van $\mathrm{Gd}^{3+}-\mathrm{AB}-\mathrm{DO} 3 \mathrm{~A}-\mathrm{SWCNTs}$ (links) en $\mathrm{Eu}^{3+}-\mathrm{AB}-\mathrm{DO} 3 \mathrm{~A}-\mathrm{SWCNTS}$ (rechts) (schaalbalk links $=50 \mathrm{~nm}$ en rechts $=20 \mathrm{~nm}$ ). Inzet in beide foto's is het EDX spectrum van de wit gemarkeerde regio's.

De eerste karakterisering en evaluatie van de eigenschappen van deze gefunctionaliseerde koolstof-gebaseerde malplaatjes laat veelbelovende resultaten zien en bevestigen/accentueren hun potentieel voor multimodale beeldvorming. Tenslotte worden de toekomstperspectieven en de uitdagingen voor de metaalcomplex-gebaseerde malplaatjes/bouwsteigers, nanostructuren en de multimodale BA's zoals beschreven in dit proefschrift kort besproken. 


\section{SUMARIO}

Imagenología molecular es una disciplina a cargo de la visualización rápida y precisa del cuerpo humano de manera, preferiblemente, no invasiva. A pesar de la gran variedad de modalidades disponibles en la actualidad, la diferenciación precisa de tejidos fisiológicamente/patológicamente diferentes sigue siendo un reto. Mientras que los agentes de imagen (Al) molecular existentes, utilizados para mejorar el contraste en una imagen sin perturbar los procesos metabólicos, han contribuido a una mejor visualización usando diferentes técnicas de imagenología, aún existen deficiencias que superar. Muchas de ellas están relacionadas a la conocida limitada resolución espacial y/o sensibilidad de la que sufren estas técnicas (Figura 1).

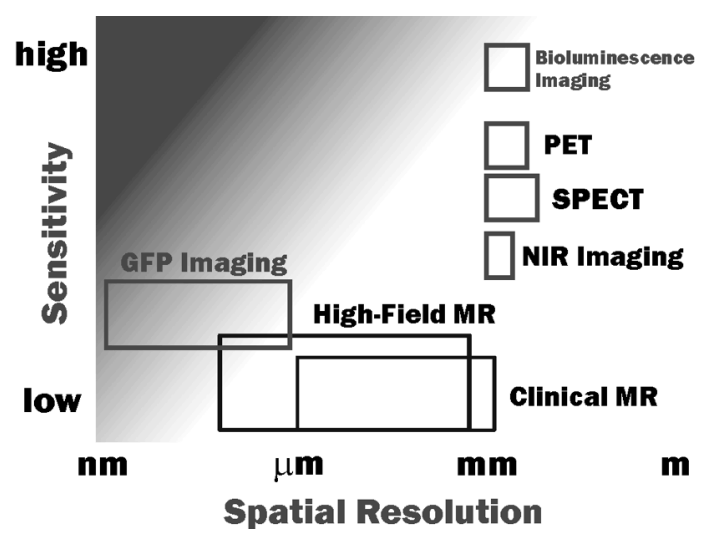

Figura 1. Sensibilidad vs. resolución espacial de algunas modalidades de imagenología. PET: tomografía por emisión de positrones. SPECT: tomografía computarizada por emisión de fotones individuales. NIR: infrarrojo cercano. MR: resonancia magnética. GFP: proteína verde fluorescente. 
Una forma común de enfrentar el problema de la baja sensibilidad y/o resolución espacial es mediante la combinación de dos (o más) modalidades de imagenología. Este campo emergente, conocido como imagenología multimodal, se dedica a la optimización de la sinergia de diferentes modalidades en un intento por obtener "lo mejor de ambos mundos". A pesar de las diferentes estrategias y combinaciones descritas en la literatura, una de las más populares emplea complejos de Gadolinio $\left(\mathrm{Gd}^{3+}\right)$ para resonancia magnética (MRI), combinados con sondas fluorescentes para su uso en imágenes ópticas. En esta tesis, nuevos enfoques dirigidos a Al simples y funcionales apropiados para imagenología multimodal empleando resonancia magnética y/o óptica, son propuestos. Los Al multimodales descritos aquí se basan en combinaciones de (nuevos) complejos metálicos a base de ligandos y nanoestructuras, donde los ligandos orgánicos no sólo sirven para la formación de complejos de metales, sino que también actúan como templados/andamios para el crecimiento de nanoestructuras.

En el Capítulo 3, la síntesis (en un paso) acuosa de nanoestructuras híbridas de plata ( $h A g N S s)$, formadas utilizando el andamio 1,4,7,10tetraaza-1-(1-aminobutil)-4,7,10-ciclododecano triacético (DO3A AB, Figura 2A), es descrita. Las nanoestructuras formadas se caracterizan y una estructura factible para estas hAgNSs es propuesta usando una amplia gama de técnicas, incluyendo espectroscopía UV-Vis, microscopía electrónica de transmisión (TEM), dispersión de luz dinámica (DLS), espectroscopía de fluorescencia (tiempo de vida y el estado de equilibrio), espectrometría de masas (MS), resonancia magnética nuclear (NMR) y espectroscopia infrarroja. Las $h \mathrm{AgNSs}$ formadas contienen dos unidades funcionales: una "gran" nanopartícula de plata (AgNP, estabilizada por los ácidos carboxílicos y el núcleo cyclen del andamio $A B-D O 3 A)$, que puede 
actuar como una plantilla para modificación de superficies y potencialmente como un agente terapéutico, y "pequeños" Ag nanoclusters $\left(\mathrm{Ag}_{\mathrm{n}} \mathrm{NCs}\right)$ que pueden ser utilizados como rastreadores ópticos debido a sus propiedades fluorescentes (Figura 2B).
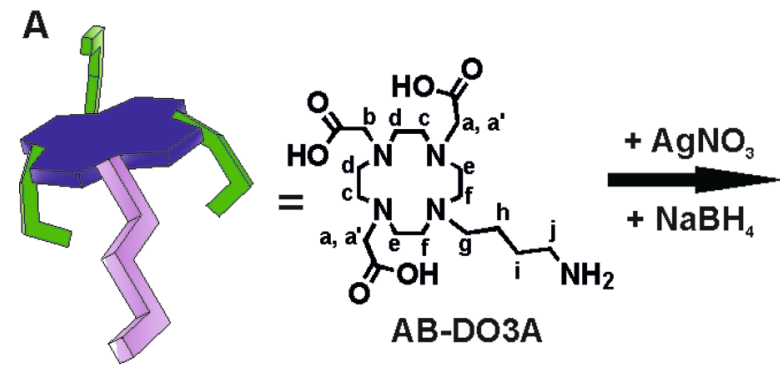

$A B-D 03 A$
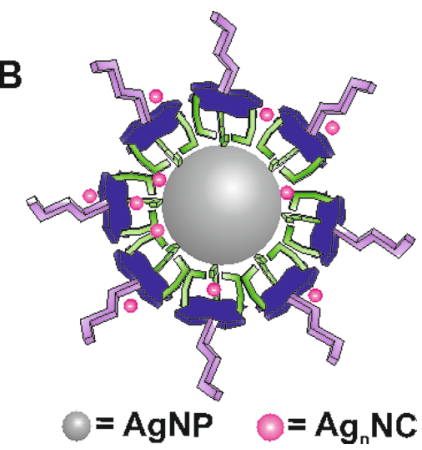

Figura 2. Nanoestructuras híbridas de plata (hAgNSs), formadas tras la reducción química de $\mathrm{AgNO}_{3}$ en presencia de $\mathrm{AB}-\mathrm{DO} 3 \mathrm{~A}$. La localización de los $\mathrm{Ag}_{\mathrm{n}} \mathrm{NC}$ dentro de la AgNP es sólo una representación esquemática.

El uso de (similares) ligandos orgánicos agregados como puntos de nucleación para la formación de jaulas de proteínas del cowpea chlorotic mottle virus (CCMV) se describe en el Capítulo 4. Jaulas moleculares estables fueron diseñadas usando puro auto-ensamblaje jerárquico de micelas del ligando 1,4,7,10-tetraza-1-(1-carboxymetilundecano) ciclododecano-4,7,10-triacético (DOTAC10) dentro del CCMV (Figura 3). DOTAC10 se utilizó para formar complejos de $\mathrm{Gd}^{3+}$, así formando micelas paramagnéticas visualizadas por MRI, o para encapsular ftalocianinas anfifílicas $\mathrm{Zn}^{2+}(\mathrm{ZnPc})$ y así confirmar ópticamente la encapsulación de las micelas. Incorporación de $\mathrm{ZnPc}$ en las micelas paramagnéticas de $\mathrm{Gd}^{3+}$ DOTAC10 resulta en cargas notablemente altas dentro de las jaulas formadas, como se observó por MRI y análisis elemental. Estas jaulas auto-ensambladas (como se confirmo por TEM y cromatografía por exclusión de tamaño) muestran altas relaxividades $r_{1}$, atribuidas a la 
agregación y alteración de la cinética de la asociación de las micelas por el confinamiento local dentro de la jaula y por la encapsulación de ZnPc.
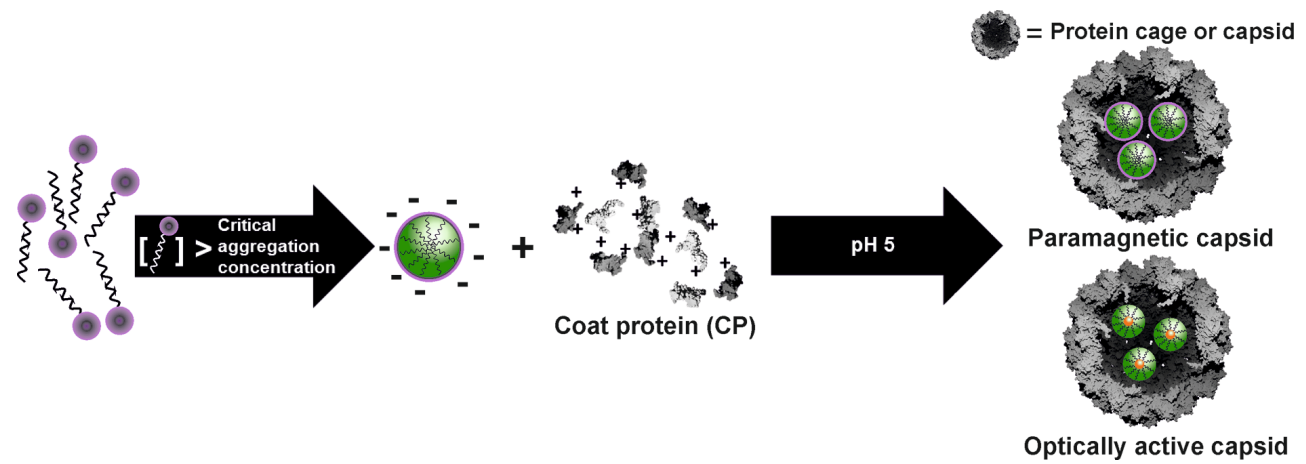

Figura 3. Representación esquemática del proceso de auto-ensamblaje desencadenado por auto-ensamblaje: micelas se forman cuando la concentración del monómero es mayor a la concentración de agregación crítica (cac). La cargas negativas de las micelas actúan como "puntos de nucleación" de auto-ensamblaje de proteínas de la cubierta (CP) del CCMV, así generando jaulas de proteínas paramagnéticas y ópticamente activas. La localización y orientación de las moléculas $\mathrm{ZnPc}$ dentro de las micelas es sólo esquemática.

En el capítulo 5, la síntesis, caracterización y estudio de la citotoxicidad de nanorods multimodales (MMAuNRs) para resonancia magnética y óptica de imagen son descritas. Las nanoestructuras de oro son modificadas por intercambio de ligandos (tioles) utilizando una relación optimizada de O-[2(3-mercaptopropionylamino)-etil]-O'-metilpolietilenglicol (PEG-SH), Gd ${ }^{3+}-1$ (11-sulfanylundecyl)-4,7,10-tris(carboximetil)-1,4,7,10-tetraaza ciclododecano $\left(\mathrm{Gd}^{3+}-\mathrm{SUDDO} \mathrm{A}\right)$ y (3aS, 6aR 4S,)-11-sulfanylundecyl 5(hexahidro-2-oxo-1H-tieno[3,4-d]imidazol-4-il) pentanoato (Biotina-SH). La posterior incubación con Cy5-estreptavidina conlleva a la formación de AuNRs paramagnéticos y fluorescentes, visualizados por microscopía de fluorescencia y resonancia magnética (Figura 4) y caracterizados usando espectroscopia UV-Vis, TEM y fluorescencia de rayos $\mathrm{X}$ por reflexión total 
(TXRF). Los MMAuNRs obtenidos con esta síntesis son biocompatibles, muestran valores de concentración letal media $\left(\mathrm{LC}_{50}\right)>150 \mathrm{pM}$ AuNR, mejores relaxividades, altas cargas de $\mathrm{Gd}^{3+}$ y puede ser ópticamente colocalizados con células C2C12 mioblastos de ratón in vitro.

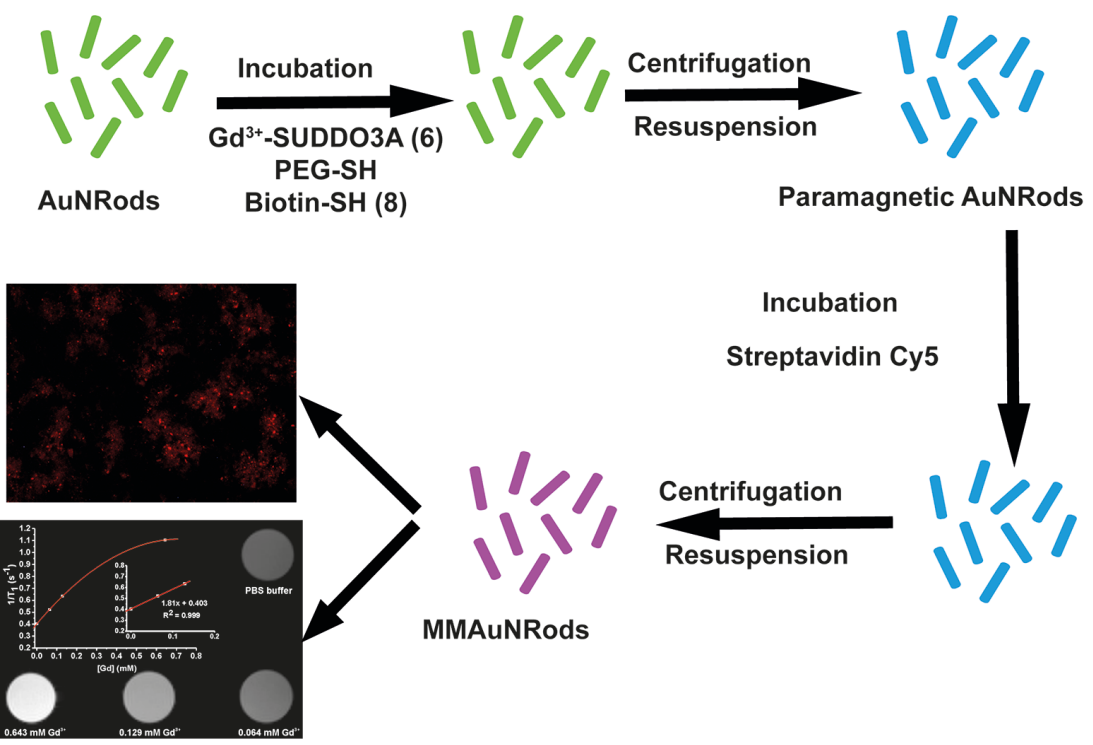

Figura 4. Representación esquemática de la síntesis de MMAuNRs. Imagen (superior) de fluorescencia: emisión roja observada, la cual corresponde a MMAuNRs etiquetados con Cy5 (tiempo de exposición 2000 ms). Imagen (baja) de MR: Imagen potenciada en $T_{1}$ de MMAuNRs a diferentes concentraciones de $\mathrm{Gd}^{3+}(600 \mathrm{MHz}, \mathrm{TE}=4 \mathrm{~ms}$, TR $=500 \mathrm{~ms})$. La gráfica muestra la relaxividad iónica $r_{1}$ correspondiente a los MMAuNRs. El grafico interno muestra el ajuste lineal del rango de concentración $0-0.13 \mathrm{mM} \mathrm{Gd}^{3+}\left(r_{1}=1.8 \mathrm{mM}^{-1} \mathrm{~s}^{-1}\right)$.

En el capítulo 6, andamios orgánicos funcionalizados a base de carbono son propuestos como plataformas para la formación de múltiples complejos de lantánidos utilizando algunos de los complejos metálicos descritos en capítulos anteriores. En primer lugar, un pequeño andamio soluble en agua que consta de un núcleo de antraceno (ANT), donde dos unidades idénticas de ligandos de 1,4,7,10-tetraazaciclododecano-1 ,4,7- 
triacético (DO3A, similar al presentado en el capítulo 5) se integran, es presentado. Las propiedades ópticas y paramagnéticas observadas en este ligando bis-macro cíclico (ANT-2DO3A, Figura 5) muestran su potencial como prueba de principio para el desarrollo de pequeños Als aprovechando las químicamente similares, pero electrónicamente/magnéticamente diferentes, propiedades de los lantánidos.
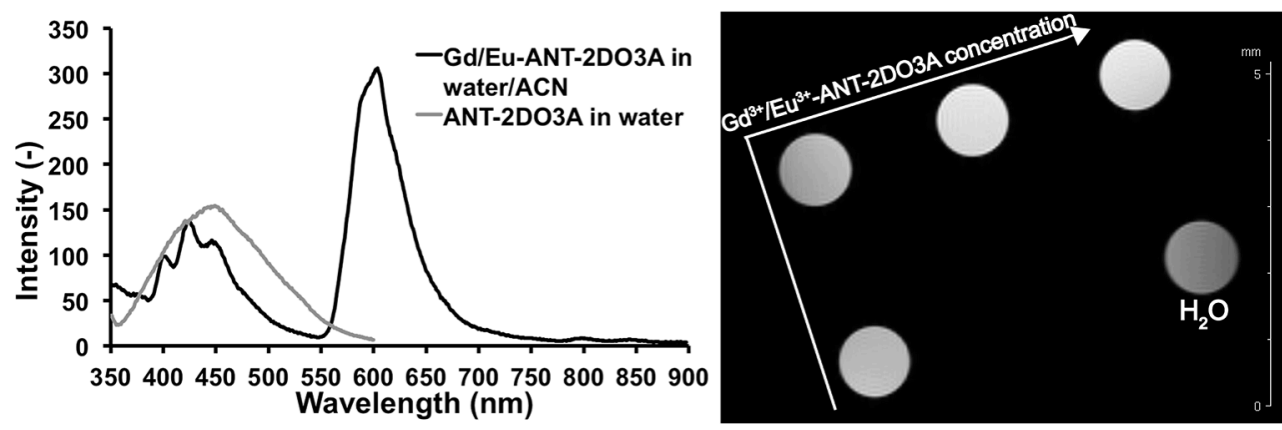

Figura 5. Izquierda: Espectros de emisión de ANT 2DO3A (línea gris, $\mathrm{H}_{2} \mathrm{O}, \lambda_{\text {exc }}=312 \mathrm{~nm}$ ) y $\mathrm{Gd}^{3+} / \mathrm{Eu}^{3+}-\mathrm{ANT}-2 \mathrm{DO} 3 \mathrm{~A}$ (línea negra, $\mathrm{H}_{2} \mathrm{O} / \mathrm{ACN}, \lambda_{\text {exc }}=262 \mathrm{~nm}$ ). Derecha: imagen potenciada en $T_{1}$ de RM que muestra varias concentraciones $(4,2,0,8$ y $0,4 \mathrm{~g} / \mathrm{L})$ de $\mathrm{Gd}^{3+} / \mathrm{Eu}^{3+}$-ANT-2DO3A y $\mathrm{H}_{2} \mathrm{O}$ (control).

Seguidamente, la unión covalente del templado AB-DO3A (Capítulo 4) a nanotubos de carbono (CNT) es explorada. CNT de pared simple (SWCNTs) son plataformas atractivas para la fijación de un gran numero de Als debido a su alta relación superficie/volumen. El enlace covalente entre AB-DO3A y los SWCNTs, el cual refuerza su solubilidad en agua con los tres ácidos carboxílicos de este ligando, seguido por la formación de complejos de lantánidos, conduce a la formación de SWCNTs funcionalizados (Figura 6). 

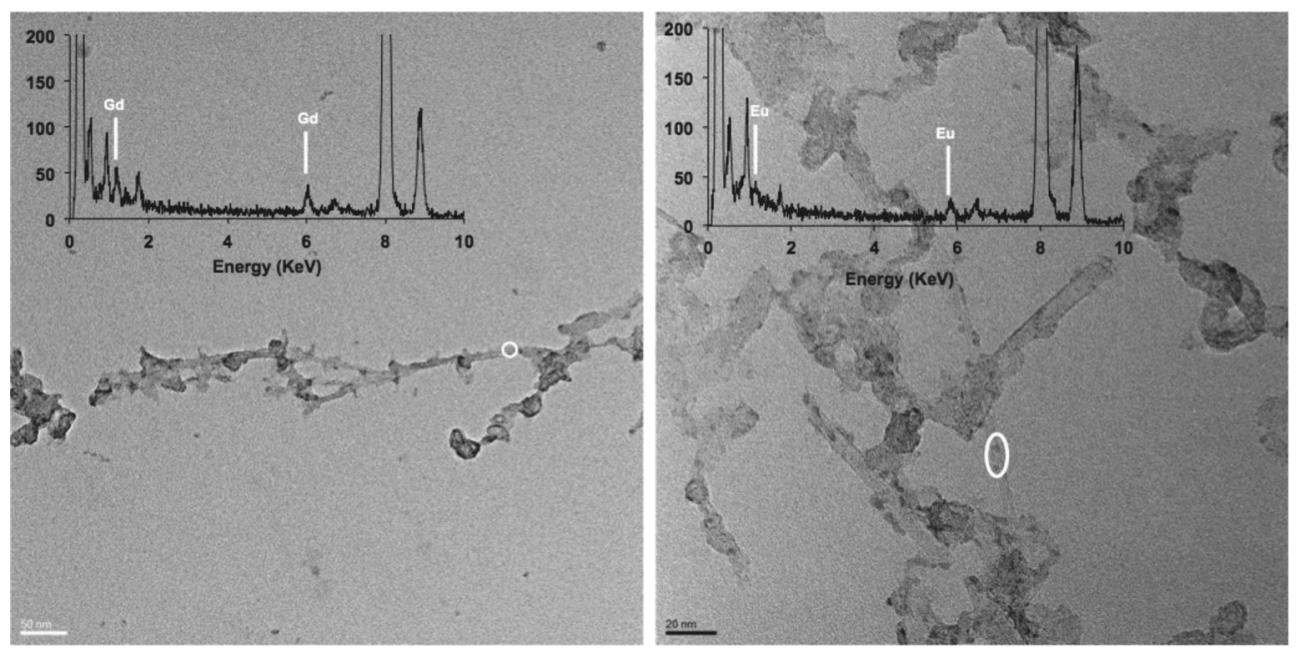

Figura 6. TEM imágenes de $\mathrm{Gd}^{3+}-\mathrm{AB}-\mathrm{DO} 3 \mathrm{~A}-\mathrm{SWCNTs}$ (izquierda) y $\mathrm{Eu}^{3+}-\mathrm{AB}-\mathrm{DO} 3 \mathrm{~A}-$ SWCNTs (derecha) (barra de escala $=50 \mathrm{~nm}$ izquierda y derecha $=20 \mathrm{~nm}$ ). Inserción en ambas imágenes es el espectro EDX de las regiones resaltadas en color blanco.

La caracterización y evaluación preliminar de las propiedades de estos andamios orgánicos a base de carbono funcionalizados muestra resultados prometedores, poniendo de manifiesto su potencial para fines de imagenología multimodal. Por último, las perspectivas de futuro y retos para estos complejos metálicos a base de templados, nanoestructuras y los Al multimodales descritos en esta tesis se discuten brevemente. 


\section{ACKNOWLEDGEMENTS}

Just like any other long story, everything must come to an end. Historically, this has always been the most "artistically" challenging part of every $\mathrm{PhD}$ thesis, with an outreach that most of us dream of for our publications. Before door is locked and the key reaches the Caribbean Sea in an SOS bottle, I would like to thank all the people who contributed to these $200+$ pages.

First of all I want to thank my promotors Prof. David Reinhoudt and Prof. Jeroen Cornelissen. David, thank you for this opportunity to work on a rather "new" subject and for giving me one of the biggest pieces of advice I have had during the toughest part of my PhD. Jeroen, I would like to acknowledge your courage by "embedding" me into your group at such a late stage during my $\mathrm{PhD}$. While in BnT I certainly had my most productive period and had the chance to work with some great people. I admire your dedication and constant struggle to combine the daily responsibilities of a small research group with an active family life. I also would like to acknowledge my daily supervisor Prof. Aldrik Velders, for the opportunity and the freedom to do research during my first PhD years. Aldrik, alles wat ik weet over NMR heb ik van jou geleerd. A special thank you to Prof. Johan Engbersen, who was my first contact with the UT 9 years ago and has actively played a role in my education all these years.

The various collaborations I had these years certainly contributed to the development of a scientifically enriched vision, which I hope is reflected in 
the various topics described in this thesis. This would have never been possible without the valuable help and intellectual input of the various collaborators I had these years. Thank you all for believing in every crazy idea or thought I had these years. First of all, I have to thank Prof. Jurriaan Huskens. Jurriaan, ik kan je niet genoeg bedanken voor jouw wettenschappelijke inzet, kritische maar toch motiverende manier van denken en, op persoonlijk niveau, alle woorden die mij steeds een hart onder de riem hebben gestoken elke keer dat het einde niet meer in zicht was. I gratefully acknowledge Prof. Jennifer Herek, whose enormous excitement about science combined with her personal way of approaching things lead to the successful end of a very challenging project. Jennifer, also you I have to thank for your time, patience and courage to embark in this project. I also thank my collaborators in Spain, especially Dr. Andrés de la Escosura. Andrés, gracias a tu motivación, interés y trabajo hemos logrado llevar este pequeño proyecto a un buen termino. I cannot forget Dr. Raluca Fratila for her unconditional support and continuous input during most of my PhD period. Ralu, muchas gracias por toda la ayuda tanto científica como personal y por leer la tesis. Estoy segura de que el futuro solo le traerá cosas buenas a una persona de tu clase científica! Michael Holtkamp and Dr. Martin Vogel from the university of Münster I thank for their help with the elemental analysis and especially Martin for his help and fantastic friendship for sooooo many years now.

I consider myself very lucky for the opportunity I had to work on exciting projects with highly skilled people. Jordi, te agradezco las buenas horas que pasamos trabajando juntos y admiro tu paciencia y dedicación a tus cultivos celulares, los cuales definitivamente añadieron algo muy positivo a esta tesis. Melanie, thank you so much for your hard work and dedication to our little project which is, in my opinion, conceptually the nicest and 
most challenging one. I could have never done this without you! Raquel, gracias por tu ayuda con las mediciones DLS y sigue adelante! I also would like to acknowledge Di Yang, Wietkse Postma and Martijn Stopel, who kindly contributed to this thesis with their expertise in optical sciences and did some of the measurements. Thank you Martin Sobik for working with me in the carbon nanotubes project (and actually providing them) and for the cognac, Drs. R. van der Hee and Tieme Stevens for some of the mass spectrometry measurements, the real senior boss of the lab Ing. Richard Egberink for his continuous and unconditional technical support, M. de Bruine for the elemental analysis and lab support, Dr. Dodo Wasserberg, Dr. Pascal Jonkheijm and Dr. Nathalie Katsonis for hours of useful discussions and my bachelor student Tom Hammer for his help with the preliminary synthetic work on the gold nanorods. Thank you Mark Smithers for the great TEM images provided and the nice chats we had during the (sometimes) very log TEM sessions. I gratefully acknowledge Dr. Roberto de la Rica for his scientific brainstorm sessions and ideas, many of them somehow reflected in this thesis. I also thank Ing. Bianca Snellink, who supported our NMR facility and was a terrific office mate. Ik mag natuurlijk niet onze eigen "dream team" van secretaressen vergeten, Izabel en Nicole (en vroeger ook Daniëlle), ik dank jullie om alle hulp en snoep die ik van jullie, vooral tijdens mijn laatste jaar, heb gekregen. Finally, a BIG BIG THANK YOU goes to Dr. Melissa Koay, who had the courage to read voluntarily (!?!?!) the entire thesis and actively contributed to two chapters. Mel, my gratitude goes to you for your help, support, friendship and our early morning jogging sessions.

I have to thank my Italian team of paranymphs, Dr. Albert Ruggi and (the soon to be Dr.) Carlo Nicosia. What an honor to have you both with me at the end of this journey! Albert, grazie mille per la amicizia di tutti questi anni. È stato un piacere lavorare con te i primi 3 anni del mio $\mathrm{PhD}$ e ho 
imparato tanto da te! Carlo, anche tu sei stato una persona veramente importante nell'ultima parte del mio $\mathrm{PhD}$ e ti ringrazio per tutte le serate bellissime che abbiamo mangiato, parlato e ti perdono se hai buttato via le chiavi della mia casa $=)$. A tutti due (+ Chiara \& Mariska) vi auguro una vita piena di amore e felicita!!!

There are a few people from the langezijds period who definitely deserve a spot here. The times I spent with two wonderful people: Nachete \& Victoria (the No More Resaca group), also Arancha, Srinidhi, Mirko, Shu-Han, Chien-Ching, Maryana, el pana Alberto, Vijay, Anna, Oya (thank you for the initial work on chapter 4), Nicolai (my one and only brozzzer), Carmen, Clemens, Joost, Miguelito San, Alfonso, Xing Yi, Martine, Dae June, Eddy, Edit, Deniz, Henk and Prof. Wim Verboom I will never forget. You all have left a footprint in my $\mathrm{PhD}$ path and I cannot express more gratitude for having spent lots of quality time with all of you. To Janet and Denis a HUGE thank you for standing by me during the very rough times I had and helping me in any way you could. To Francesca and Riccardo, who still provide me with some wonderful memories every time we meet somewhere in this world. Roald, bedankt voor ALLES wat jij hebt gedaan (vriend, koffie drinker, taxi chauffeur, office boy, psycholoog, etc...) en alle steun van de laatste jaren! Merci Severine for the nice "wine and olives" evenings we had. I cannot forget my dear friend Dr. Muddassir a.k.a. "pough", who showed me just how two very different people could develop a nice, constructive friendship (oh! and thanks for the sweet rice recipe!!!). I cannot forget the "new" people, Anne (bedankt voor de dozen!!!), Martijn, Rick \& Rik, Sven, Roberto, Emanuela, Sarah, Supitchaya, Ye, Rajesh, Vishwas, Xuemei, Angel, Tom, Janneke, Timon, Tibor en Jasper. Finally, thank you Kim for all the wonderful memories and good (and at times very bad) times! 
No puedo olvidar a la comunidad latina que ha sido de vital importancia todos estos años! Naye \& Eduardo, David (mi comandante), Maite \& Jorge, Juan \& Diru + Nico, Daniela \& Oscar, Julián, Mario, Maryana, Vicky \& Lorenzo....gracias por las innumerables cenas juntos y por el techo que me dieron cuando lo necesite!!! Muchas gracias también a los miembros de LA Voz, Andrea, Coen, Nayeli, Joni, David, Mario, Laura, Oscar \& Daniela, con quienes trabaje durante el primer ano de vida de la asociación. It wouldn't be fair to forget the Delft community, the only real rock star of the ChemE Dr. Corradijntje Locati(e), the careless whisperer Mario Valvo and Christophe and Dainius, who were always ready for a nice dinner and a chat.

Ik wil ook graag mijn yoga leraressen Henriëtte, Susanne, Jeanette en Marlies bedanken, die mij enorm hebben geholpen en een bron van inspiratie zijn geweest. Natuurlijk mag ik mijn fysiotherapeut Anneloes niet vergeten, die elke maand mijn zwakke rug wist te verhelpen. Uiteraard wil ik mijn "Nederlandse familie" bedanken, Jan, Bea, Ralph, Ruurd \& Lenie, die mij de beste inburgeringscursus in Nederland hebben gegeven en mij zoveel jaren hebben gesteund en geholpen. Ik hoop dat jullie trots op het resultaat zijn!

Durante mis anos de bachillerato fue claro que tenia una inclinación por las ciencias. Gracias a mis profesores Gustavo Guerra (física) y Carlos Brito (química) logre desarrollar varias habilidades que me ayudaron en mis años siguientes. Cabe destacar que Carlos Brito no solo me introdujo al mundo de los factores de conversión en química, sino que también me dio mi primera copia de El Extranjero de Albert Camus.

A mis amigos de siempre Ana, Daniela, Elayne, Bea, George, Valeska Maria, Jorge \& Caco, que aunque estemos repartidos por el mundo siempre logramos vernos. Sin mas, debo agradecer a mi familia por todo el apoyo que me han brindado. A mis padres Itamar y Jealemy, quienes 
espero estén orgullosos de mi no por haber llegado al final de este periodo y el titulo que llevaré, sino por haber criado a un ser con pensamiento critico e independiente. Que eso lo hicieron en un país donde tuvieron que enfrentar muchas dificultades sociales, les da el estatus de "super papas". A mis hermanos Raúl y Pedro, mis tíos Alejandro y Gladys, mis primas Angela y Alejandra (rata mayor) y a los nuevos miembros Eva, Alejandra y Mathias, quienes desde la distancia siempre estuvieron a mi lado. Cristian, no hay palabras suficientes para expresarte mi gratitud por todo lo que haz hecho durante estos últimos anos. Sin tu apoyo esto nunca hubiera sido posible, fuistes la fuerza que necesitaba en los momentos que pensé que ya no podía mas. Parte de esta tesis te pertenece a ti, literalmente ya que tu contribución intelectual en varios de los capítulos no puede ser negada, y espero que el resultado sea algo de lo que puedas estar orgulloso. Simbólicamente, el final de este ano será el inicio de un nuevo periodo...estoy curiosa que pasará!

"Happiness only real when shared" Alexander Supertramp 


\section{ABOUT THE AUTHOR}

Jealemy Galindo Millán was born on March 9th, 1981 in Caracas (Venezuela). After finishing high school, she started her studies in chemical engineering at the Universidad Central de Venezuela (UCV), where she studied for three years and worked as an assistant for programming courses at the faculty of engineering. At the end of 2001, she moved to the Netherlands and enrolled at the University of Twente in 2003 to continue her studies. During this period she lost her interest in programming and became interested in chemical analysis and biomedical technology, where she minored in 2005. In September of that same year she obtained her bachelor degree at the same university after working at the Chemical Analysis group of Prof. Uwe Karst, where she studied the screening of carbonyl compounds in automobile exhausts using active sampling methods and LC-MS. In November 2007 she obtained her degree (cum laude) in chemistry and technology of materials at the University of Twente. During her master thesis she studied the possible limitations and applications of high-field (micro) magnetic resonance imaging $(\mu \mathrm{MRI})$. This work was carried out at the Supramolecular Chemistry and Technology (SMCT) group of Prof. David N. Reinhoudt, under the supervision of Dr. Aldrik Velders. In the same period she received the Unilever Research Prize 2007 for her master thesis. In December 2007 she started her PhD research in the SMCT group, and later became a member of the Biomolecular Nanotechnology (BnT) group of Prof. Jeroen Cornelissen at the University of Twente. The results of this research are presented in this thesis. From November 2012 she is a postdoctoral fellow in the group of Prof. Leonard Prins at the Università degli studi di Padova, Italy. 